\title{
The enigma of lymph node staging in breast cancer
}

Citation for published version (APA):

van Nijnatten, TJA. (2017). The enigma of lymph node staging in breast cancer. [Doctoral Thesis, Maastricht University]. Datawyse / Universitaire Pers Maastricht. https://doi.org/10.26481/dis.20171221tvn

Document status and date:

Published: 01/01/2017

DOI:

10.26481/dis.20171221tvn

Document Version:

Publisher's PDF, also known as Version of record

\section{Please check the document version of this publication:}

- A submitted manuscript is the version of the article upon submission and before peer-review. There can be important differences between the submitted version and the official published version of record.

People interested in the research are advised to contact the author for the final version of the publication, or visit the DOI to the publisher's website.

- The final author version and the galley proof are versions of the publication after peer review.

- The final published version features the final layout of the paper including the volume, issue and page numbers.

Link to publication

\footnotetext{
General rights rights.

- You may freely distribute the URL identifying the publication in the public portal. please follow below link for the End User Agreement:

www.umlib.nl/taverne-license

Take down policy

If you believe that this document breaches copyright please contact us at:

repository@maastrichtuniversity.nl

providing details and we will investigate your claim.
}

Copyright and moral rights for the publications made accessible in the public portal are retained by the authors and/or other copyright owners and it is a condition of accessing publications that users recognise and abide by the legal requirements associated with these

- Users may download and print one copy of any publication from the public portal for the purpose of private study or research.

- You may not further distribute the material or use it for any profit-making activity or commercial gain

If the publication is distributed under the terms of Article $25 \mathrm{fa}$ of the Dutch Copyright Act, indicated by the "Taverne" license above, 


\section{The enigma of lymph node staging in breast cancer}


Parts of the research described in this thesis were financially supported by grants from Kankeronderzoekfonds Limburg, Carla Boetes Fund, Alpe d'Huzes Foundation and KWF Kankerbestrijding.

(c) Thiemo van Nijnatten, Maastricht 2017

No part of this book may be reproduced or transmitted in any form or by any means, without prior permission in writing by the author, or when appropriate, by the publishers of the publications.

Cover design: Koen Kuijper, photo by Carlijn van Nijnatten-Giesbers

Layout: Tiny Wouters

Production: Datawyse, Universitaire Pers Maastricht

ISBN $\quad 9789461597731$

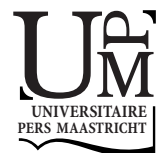

The publication of this thesis was financially supported by Clinical Trial Center Maastricht B.V. and Maastricht University. 


\title{
The enigma of lymph node staging in breast cancer
}

\author{
PROEFSCHRIFT
}

ter verkrijging van de graad van doctor aan de Universiteit Maastricht, op gezag van de Rector Magnificus, Prof. dr. Rianne M. Letschert, volgens het besluit van het College van Decanen, in het openbaar te verdedigen op donderdag 21 december 2017 om 10.00 uur

door

Thiemo Jacobus Antonius van Nijnatten 


\section{Promotor:}

Prof. dr. R.G.H. Beets-Tan

\section{Copromotores:}

Dr. M.L. Smidt

Dr. M.B.I. Lobbes

\section{Beoordelingscommissie:}

Prof. dr. S.G.F. Robben (voorzitter)

Prof. dr. L.J. Boersma

Prof. dr. R.M. Pijnappel (Universiteit Utrecht)

Prof. dr. F.C.S. Ramaekers

Prof. dr. E.J.T. Rutgers (Universiteit van Amsterdam) 


\section{Contents}

Chapter 1 Introduction and outline of the thesis

Part I Invasive lymph node staging

Chapter 2 The diagnostic performance of sentinel lymph node biopsy in pathologically confirmed node positive breast cancer patients after neoadjuvant systemic therapy: a systematic review and meta-analysis

Chapter 3 A novel less invasive approach for axillary staging after neoadjuvant chemotherapy in patients with axillary node positive breast cancer by combining Radioactive Iodine Seed localization in the Axilla with the Sentinel node procedure (RISAS):

a Dutch prospective multicentre validation study

Part II Non-invasive lymph node staging

Chapter 4 Routine use of standard breast MRI compared to axillary ultrasound for differentiating between no, limited and advanced axillary nodal disease in newly diagnosed breast cancer patients

Chapter 5 The diagnostic performance of gadofosveset-enhanced axillary MRI for nodal (re)staging in breast cancer patients: results of a validation study

Chapter 6 Added value of dedicated axillary hybrid 18F-FDG PET/MRI for nodal staging in clinically node positive breast cancer patients - a feasibility study

\section{Part III Classification of lymph node staging}

Chapter 7 TNM classification and the need for revision of $\mathrm{pN}_{3}$ a breast cancer

Chapter 8 Prognosis of residual axillary disease after neoadjuvant chemotherapy in clinically node positive breast cancer patients: isolated tumor cells and micrometastases carry a better prognosis than macrometastases

Chapter 9 Does the TNM classification of solitary internal mammary lymph node metastases in breast cancer still apply? 
Summary

Samenvatting 169

Valorisation

175

List of publications

181

Dankwoord

187

Curriculum Vitae

193 


\section{Chapter 1}

\section{Introduction}





\section{Introduction}

Breast cancer is the most common type of invasive cancer among women worldwide, with an estimated 1.7 million cases and 521,900 deaths in 2012. ${ }^{1}$ In the Netherlands, more than 14,00o patients are newly diagnosed with breast cancer each year. ${ }^{2}$ Nowadays, standard treatment consists of an operation of the breast and axilla, complemented with radiation and/or systemic therapy. Obviously, survival and reducing recurrence are the most important goals. Due to this extensive treatment, mean five year overall survival has increased over the last decades to $90 \%{ }^{3}$ The in general excellent survival caused an attention shift from merely survival to the presence of morbidity and quality of life after breast cancer treatment. Better patient selection for specific therapies and use of less invasive techniques, in order to avoid overtreatment on an individual level, aim to further minimize morbidity and complications of therapy.

Axillary lymph nodes are examined at breast cancer diagnosis, because of their prognostic relevance. Axillary lymph node dissection (ALND), i.e. the complete removal of all axillary lymph nodes, used to be the surgical treatment of choice to provide accurate information about actual lymph node status for a long period. Eventually, approximately $30-40 \%$ of all patients had axillary lymph node metastases. ${ }^{4}$ Hence, $60-70 \%$ of all patients underwent ALND without having any clinical or prognostic benefit of the surgical procedure. However, ALND causes long-term morbidities in up to $45 \%$ of patients, such as lymphedema, nerve injury and reduced shoulder function. ${ }^{5}$ A less invasive procedure to identify malignant lymph nodes was therefore needed.

In 1994 sentinel lymph node biopsy (SLNB) was introduced as a less invasive technique to evaluate the axillary lymph node status in clinically node negative patients. ${ }^{6}$ The principle of SLNB relies on the lymphatic drainage from the primary tumour in the breast to one or more regional lymph nodes, assuming that if these lymph nodes contain no tumour cells, all other regional lymph nodes can be considered benign. In case of sentinel lymph node metastases, a completion ALND was performed. Several studies confirmed the safety and clinical applicability of SLNB as less invasive procedure for nodal staging in clinically node negative patients. ${ }^{7,8}$ Yet, complications of SLNB still occur, like lymphedema, numbness and pain, albeit less frequently than in ALND. ${ }^{9}$

In the Netherlands, standard axillary examination consists of an ultrasound (US) with tissue sampling (if indicated) to identify clinically node positive patients. ${ }^{10,11}$ A previous study demonstrated that US can identify less than $50 \%$ of the patients with axillary lymph node metastases. ${ }^{12}$ Yet, in patients who are 
considered clinically node positive (based on US findings) ALND is performed directly, which prevents these patients undergoing two surgical procedures (SLNB followed by completion ALND).

The hypothesis of detecting every positive lymph node in order to improve prognosis was reconsidered in 2011. The ACOSOG Zoon trial, a randomized clinical trial which included clinically node negative breast cancer patients (based on physical examination) treated with breast conserving therapy, demonstrated that in case of one or two positive lymph nodes after sentinel lymph node biopsy there was no prognostic benefit of performing a completion axillary lymph node dissection or not. ${ }^{13,14}$ These results were confirmed by the IBCSG 23-01 and AATRM 048/13/200o trials in which patients with one to three positive nodes were investigated..$^{15,16}$ In summary, excluding advanced axillary nodal disease (i.e. more than three positive nodes) rather than detecting clinically positive nodes is becoming more and more important. Previous studies have demonstrated that axillary US is an excellent imaging modality to exclude advanced axillary nodal disease in case of negative findings. ${ }^{12,17}$ However, in case of positive findings US is unable to differentiate between limited (i.e. one to three positive nodes) and advanced axillary nodal disease. ${ }^{12}$

If a non-invasive technique would be able to accurately differentiate between patients with and without axillary lymph node metastases, axillary surgery could be safely omitted in case of node negative findings. This would be of added value for many patients, as SLNB and its subsequent morbidity could be omitted in all node negative patients then. Current imaging modalities like US, magnetic resonance imaging (MRI) and positron emission tomography/computed tomography (PET/CT) are insufficiently accurate to differentiate between node negative and positive findings, with previously reported percentages of $25 \%$ $(=52 / 208), 24 \%(82 / 337)$, and $47 \%(=42 / 90)$ of patients still containing axillary lymph node metastases in case of negative findings. ${ }^{17-19}$ Regarding MRI for axillary nodal staging, diagnostic performance improved with the use of a dedicated axillary MR protocol. ${ }^{20}$ In addition, a feasibility study concerning gadofosvesetenhanced dedicated axillary MRI in breast cancer patients demonstrated promising results for axillary nodal staging, with a reported sensitivity of $86 \%$, specificity of $94 \%$, positive predictive value (PPV) of $75 \%$ and negative predictive value (NPV) of $97 \%$, on a node-by-node analysis. ${ }^{21}$ However, these results were based on a small sample size of just ten patients, which demands further studies to confirm these results.

Since 1958 the definitive histopathological result of surgical treatment in breast cancer patients is classified according to the TNM classification. ${ }^{22}$ This 
staging system defines the extent of primary tumour ( $\mathrm{T}$ status), the extent of nodal disease ( $\mathrm{N}$ status), and the presence of distant metastases ( $\mathrm{M}$ status). The TNM classification is regularly updated by the International Union against Cancer, with a view to maintain a clinically relevant classification system. ${ }^{23}$ The $\mathrm{N}$ status represents the number, size and location of regional lymph node metastases. In clinical practice, TNM classification is often used to decide which type of additional treatment patients will receive. For instance radiotherapy indications and the use of adjuvant systemic therapy, can be decided based upon these results." The existence of such a dedicated classification system underlines the importance of accurate clinical lymph node staging prior to therapy initiation in breast cancer patients.

Besides irradiation and surgical treatment, systemic therapy has an important role in treatment of breast cancer patients. Since the introduction of chemotherapy in the $20^{\text {th }}$ century, administration of chemotherapy has shown substantial improvement in patient's prognosis. ${ }^{24,25}$ The indication for systemic therapy depends on several criteria, for instance tumour biology, size, grade and presence and number of lymph node metastases. ${ }^{26}$ Earlier, systemic therapy was applied after the operation of breast and axilla, the so called 'adjuvant setting'. In recent years, systemic therapy is more and more administered prior to surgery, i.e. 'neoadjuvant setting. ${ }^{27,28}$ This might result in regression of tumoursize or even a pathological complete response ( $\mathrm{pCR}$, i.e. no residual tumour present after treatment). Neoadjuvant systemic therapy might result in smaller breastconserving therapy, breast-conserving therapy where initially mastectomy was preferred, as well as in vivo tumour response monitoring. ${ }^{29,30}$ Neoadjuvant systemic therapy allows axillary lymph node metastases to convert to axillary pCR as well, making the ALND a redundant operation. According to recent studies, axillary pCR appears in $20-60 \%$ of all clinically node positive patients, depending on tumour biology..$^{31}$ Furthermore, identification of patients whose axillary lymph node metastases converted to axillary $\mathrm{PCR}$ is important since it is a sign of extreme chemosensitivity. ${ }^{32,33}$ So far, no non-invasive technique has shown accurate results to discriminate axillary $\mathrm{pCR}$ from remaining lymph node metastases after neoadjuvant systemic therapy. ${ }^{34,35}$ A less invasive technique as opposed to ALND is therefore warranted in order to identify axillary pCR in clinically node positive patients treated with neoadjuvant systemic therapy. 


\section{Aims and outline of this thesis}

The overall aim is to investigate the enigma of lymph node staging in breast cancer patients, by improving the diagnostic accuracy of nodal staging in order to achieve a more patient-tailored treatment with minimal invasive therapy and reduced morbidity. This thesis consists of three parts concerning lymph node staging in breast cancer: invasive, non-invasive and classification.

\section{Part I - Invasive lymph node staging}

Chapter 2 is a meta-analysis on the sentinel lymph node biopsy as axillary staging technique in clinically node positive patients treated with neoadjuvant systemic therapy. Chapter 3 describes the RISAS-procedure (defined as a combination of the sentinel lymph node biopsy and an iodine seed placement and removal in the pathologically confirmed axillary lymph node metastasis) for axillary staging in clinically node positive breast cancer patients after neoadjuvant systemic therapy.

\section{Part II - Non-invasive lymph node staging}

Chapter 4 investigates the diagnostic performance of standard $\mathrm{T}_{2}$ weighted breast MRI compared to axillary US to differentiate between patients with no (pNo), limited $\left(\mathrm{pN}_{1}\right)$ and advanced $\left(\mathrm{pN}_{2}-3\right)$ axillary nodal disease. In chapter 5 a validation study examines the diagnostic performance of gadofosveset-enhanced axillary MRI in breast cancer patients. Chapter 6 assesses the added value of dedicated axillary hybrid 18F-FDG PET/MRI for lymph node staging in clinically node positive breast cancer patients.

\section{Part III - Classification of lymph node staging}

Chapter 7 evaluates the classification of breast cancer patients with a $\mathrm{pN}_{3} \mathrm{a}$ nodal status (at least one infraclavicular lymph node metastases or ten or more axillary lymph node metastases). In chapter 8 the prognosis and classification of residual axillary disease (isolated tumour cells, micrometastases or macrometastases) is studied in clinically node positive breast cancer patients treated with neoadjuvant chemotherapy. The TNM classification of solitary internal mammary lymph node metastases is examined in chapter 9 . 


\section{References}

1. Torre LA, Bray F, Siegel RL, Ferlay J, Lortet-Tieulent J, Jemal A. Global cancer statistics, 2012. CA Cancer J Clin 2015;65(2):87-108.

2. IKNL. Cijfers over Kanker: incidentie borstkanker. Integraal Kankercentrum Nederland. 2015; http://www.cijfersoverkanker.nl/selecties/Dataset_1/img58c7d92238216. Accessed March 2017.

3. Mariotto AB, Noone AM, Howlader N, Cho H, Keel GE, Garshell J, Woloshin S, Schwartz LM. Cancer survival: an overview of measures, uses, and interpretation. J Natl Cancer Inst Monogr 2014;2014(49):145-86.

4. DeSantis CE, Fedewa SA, Goding Sauer A, Kramer JL, Smith RA, Jemal A. Breast cancer statistics, 2015: Convergence of incidence rates between black and white women. CA Cancer J Clin 2016; 66(1):31-42.

5. Ahmed RL, Prizment A, Lazovich D, Schmitz KH, Folsom AR. Lymphedema and quality of life in breast cancer survivors: the Iowa Women's Health Study. J Clin Oncol 20o8;26(35):5689-96.

6. Giuliano AE, Dale PS, Turner RR, Morton DL, Evans SW, Krasne DL. Improved axillary staging of breast cancer with sentinel lymphadenectomy. Ann Surg 1995;222(3):394-9; discussion 399-401.

7. Veronesi U, Paganelli G, Viale G, Luini A, Zurrida S, Galimberti V, Intra M, Veronesi P, Robertson C, Maisonneuve P, Renne G, De Cicco C, De Lucia F, Gennari R. A randomized comparison of sentinel-node biopsy with routine axillary dissection in breast cancer. N Engl J Med 2003;349(6): 546-53.

8. Krag DN, Anderson SJ, Julian TB, Brown AM, Harlow SP, Ashikaga T, Weaver DL, Miller BJ, Jalovec LM, Frazier TG, Noyes RD, Robidoux A, Scarth HM, Mammolito DM, McCready DR, Mamounas EP, Costantino JP, Wolmark N, National Surgical Adjuvant, B, Bowel P. Technical outcomes of sentinel-lymph-node resection and conventional axillary-lymph-node dissection in patients with clinically node-negative breast cancer: results from the NSABP B-32 randomised phase III trial. Lancet Oncol 2007;8(10):881-8.

9. Lucci A, McCall LM, Beitsch PD, Whitworth PW, Reintgen DS, Blumencranz PW, Leitch AM, Saha S, Hunt KK, Giuliano AE, American College of Surgeons Oncology G. Surgical complications associated with sentinel lymph node dissection (SLND) plus axillary lymph node dissection compared with SLND alone in the American College of Surgeons Oncology Group Trial Zoo11. J Clin Oncol 2007;25(24):3657-63.

10. NABON (2012). National breast cancer guideline, Oncoline. http://www.oncoline.nl/ mammacarcinoom. Accessed March 2017.

11. Senkus E, Kyriakides S, Ohno S, Penault-Llorca F, Poortmans P, Rutgers E, Zackrisson S, Cardoso F, Committee EG. Primary breast cancer: ESMO Clinical Practice Guidelines for diagnosis, treatment and follow-up. Ann Oncol 2015;26 Suppl 5:v8-30.

12. Schipper RJ, van Roozendaal LM, de Vries B, Pijnappel RM, Beets-Tan RG, Lobbes MB, Smidt ML. Axillary ultrasound for preoperative nodal staging in breast cancer patients: is it of added value? Breast 2013;22(6):1108-13.

13. Giuliano AE, Hunt KK, Ballman KV, Beitsch PD, Whitworth PW, Blumencranz PW, Leitch AM, Saha S, McCall LM, Morrow M. Axillary dissection vs no axillary dissection in women with invasive breast cancer and sentinel node metastasis: a randomized clinical trial. JAMA 2011; 305(6):569-75.

14. Giuliano AE, Ballman K, McCall L, Beitsch P, Whitworth PW, Blumencranz P, Leitch AM, Saha S, Morrow M, Hunt KK. Locoregional Recurrence After Sentinel Lymph Node Dissection With or Without Axillary Dissection in Patients With Sentinel Lymph Node Metastases: Long-term Follow-up From the American College of Surgeons Oncology Group (Alliance) ACOSOG Zoon Randomized Trial. Ann Surg 2016;264(3):413-20. 
15. Galimberti V, Cole BF, Zurrida S, Viale G, Luini A, Veronesi P, Baratella P, Chifu C, Sargenti M, Intra M, Gentilini O, Mastropasqua MG, Mazzarol G, Massarut S, Garbay JR, Zgajnar J, Galatius H, Recalcati A, Littlejohn D, Bamert M, Colleoni M, Price KN, Regan MM, Goldhirsch A, Coates AS, Gelber RD, Veronesi U, International Breast Cancer Study Group Trial i. Axillary dissection versus no axillary dissection in patients with sentinel-node micrometastases (IBCSG 23-01): a phase 3 randomised controlled trial. Lancet Oncol 2013; 14(4):297-305.

16. Sola M, Alberro JA, Fraile M, Santesteban P, Ramos M, Fabregas R, Moral A, Ballester B, Vidal S. Complete axillary lymph node dissection versus clinical follow-up in breast cancer patients with sentinel node micrometastasis: final results from the multicenter clinical trial AATRM 048/13/200o. Ann Surg Oncol 2013;20(1):120-7.

17. Neal CH, Daly CP, Nees AV, Helvie MA. Can preoperative axillary US help exclude $\mathrm{N}_{2}$ and $\mathrm{N}_{3}$ metastatic breast cancer? Radiology 2010;257(2):335-41.

18. Hieken TJ, Trull BC, Boughey JC, Jones KN, Reynolds CA, Shah SS, Glazebrook KN. Preoperative axillary imaging with percutaneous lymph node biopsy is valuable in the contemporary management of patients with breast cancer. Surgery 2013;154(4):831-8; discussion 838-40.

19. Koolen BB, Valdes Olmos RA, Elkhuizen PH, Vogel WV, Vrancken Peeters MJ, Rodenhuis S, Rutgers EJ. Locoregional lymph node involvement on 18F-FDG PET/CT in breast cancer patients scheduled for neoadjuvant chemotherapy. Breast Cancer Res Treat 2012;135(1):231-40.

2o. Kuijs VJ, Moossdorff M, Schipper RJ, Beets-Tan RG, Heuts EM, Keymeulen KB, Smidt ML, Lobbes MB. The role of MRI in axillary lymph node imaging in breast cancer patients: a systematic review. Insights Imaging 2015;6(2):203-15.

21. Schipper RJ, Smidt ML, van Roozendaal LM, Castro CJ, de Vries B, Heuts EM, Keymeulen KB, Wildberger JE, Lobbes MB, Beets-Tan RG. Noninvasive nodal staging in patients with breast cancer using gadofosveset-enhanced magnetic resonance imaging: a feasibility study. Invest Radiol 2013;48(3):134-9.

22. UICC Committee on Clinical Stage Classification and Applied Statistics. Clinical stage classification and presentation of results, malignant tumors of the breast and larynx. International Union Against Cancer, Paris; 1958.

23. Singletary SE, Greene FL, Breast Task F. Revision of breast cancer staging: the 6th edition of the TNM Classification. Semin Surg Oncol 2003;21(1):53-9.

24. DeVita VT, Jr, Chu E. A history of cancer chemotherapy. Cancer Res 20o8;68(21):8643-53.

25. Fisher B, Ravdin RG, Ausman RK, Slack NH, Moore GE, Noer RJ. Surgical adjuvant chemotherapy in cancer of the breast: results of a decade of cooperative investigation. Ann Surg 1968;168(3): 337-56.

26. Goldhirsch A, Ingle JN, Gelber RD, Coates AS, Thurlimann B, Senn HJ, Thresholds for therapies: highlights of the St Gallen International Expert Consensus on the primary therapy of early breast cancer 2009. Ann Oncol 2009;20(8):1319-29.

27. Gralow JR, Burstein HJ, Wood W, Hortobagyi GN, Gianni L, von Minckwitz G, Buzdar AU, Smith IE, Symmans WF, Singh B, Winer EP. Preoperative therapy in invasive breast cancer: pathologic assessment and systemic therapy issues in operable disease. J Clin Oncol 2008;26(5):814-9.

28. Kaufmann M, von Minckwitz G, Mamounas EP, Cameron D, Carey LA, Cristofanilli M, Denkert C, Eiermann W, Gnant M, Harris JR, Karn T, Liedtke C, Mauri D, Rouzier R, Ruckhaeberle E, Semiglazov V, Symmans WF, Tutt A, Pusztai L. Recommendations from an international consensus conference on the current status and future of neoadjuvant systemic therapy in primary breast cancer. Ann Surg Oncol 2012;19(5):1508-16.

29. Mieog JS, van der Hage JA, van de Velde CJ. Neoadjuvant chemotherapy for operable breast cancer. Br J Surg 2007;94(10):1189-200.

3o. Marinovich ML, Houssami N, Macaskill P, Sardanelli F, Irwig L, Mamounas EP, von Minckwitz G, Brennan ME, Ciatto S. Meta-analysis of magnetic resonance imaging in detecting residual breast cancer after neoadjuvant therapy. J Natl Cancer Inst 2013;105(5):321-33. 
31. Boughey JC, McCall LM, Ballman KV, Mittendorf EA, Ahrendt GM, Wilke LG, Taback B, Leitch AM, Flippo-Morton T, Hunt KK. Tumor biology correlates with rates of breast-conserving surgery and pathologic complete response after neoadjuvant chemotherapy for breast cancer: findings from the ACOSOG Z1071 (Alliance) Prospective Multicenter Clinical Trial. Ann Surg 2014;26o(4):608-14; discussion 614-6.

32. von Minckwitz G, Untch M, Blohmer JU, Costa SD, Eidtmann H, Fasching PA, Gerber B, Eiermann W, Hilfrich J, Huober J, Jackisch C, Kaufmann M, Konecny GE, Denkert C, Nekljudova V, Mehta K, Loibl S. Definition and impact of pathologic complete response on prognosis after neoadjuvant chemotherapy in various intrinsic breast cancer subtypes. J Clin Oncol 2012;30(15): 1796-804.

33. Cortazar P, Zhang L, Untch M, Mehta K, Costantino JP, Wolmark N, Bonnefoi H, Cameron D, Gianni L, Valagussa P, Swain SM, Prowell T, Loibl S, Wickerham DL, Bogaerts J, Baselga J, Perou C, Blumenthal G, Blohmer J, Mamounas EP, Bergh J, Semiglazov V, Justice R, Eidtmann H, Paik S, Piccart M, Sridhara R, Fasching PA, Slaets L, Tang S, Gerber B, Geyer CE, Jr, Pazdur R, Ditsch N, Rastogi P, Eiermann W, von Minckwitz G. Pathological complete response and long-term clinical benefit in breast cancer: the CTNeoBC pooled analysis. Lancet 2014;384(9938):164-72.

34. Schipper RJ, Moossdorff M, Beets-Tan RG, Smidt ML, Lobbes MB. Noninvasive nodal restaging in clinically node positive breast cancer patients after neoadjuvant systemic therapy: a systematic review. Eur J Radiol 2015;84(1):41-7.

35. Schwentner L, Helms G, Nekljudova V, Ataseven B, Bauerfeind I, Ditsch N, Fehm T, Fleige B, Hauschild M, Heil J, Kummel S, Lebeau A, Schmatloch S, Schrenk P, Staebler A, Loibl S, Untch M, Von Minckwitz G, Liedtke C, Kuhn T. Using ultrasound and palpation for predicting axillary lymph node status following neoadjuvant chemotherapy - Results from the multi-center SENTINA trial. Breast 2017;31:202-7. 



\section{Part I}

\section{Invasive lymph node staging}





\section{Chapter

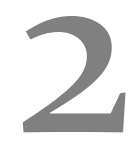

The diagnostic performance of sentinel lymph node biopsy in pathologically confirmed node positive breast cancer patients after neoadjuvant systemic therapy: A systematic review and meta-analysis

TJA van Nijnatten, RJ Schipper, MBI Lobbes, PJ Nelemans, RGH Beets-Tan, ML Smidt Eur J Surg Oncol. 2015;41(10):1278-1287 


\section{Abstract}

\section{Purpose}

To provide a systematic review and meta-analysis of studies investigating sentinel lymph node biopsy after neoadjuvant systemic therapy in pathologically confirmed node positive breast cancer patients.

\section{Methods}

Pubmed and Embase databases were searched until June 19th, 2015. All abstracts were read and data extraction was performed by two independent readers. A random-effects model was used to pool the proportion for identification rate, false-negative rate (FNR) and axillary pCR with 95\% confidence intervals. Subgroup analyses affirmed potential confounders for identification rate and FNR.

\section{Results}

A total of 997 abstracts were identified and eventually eight studies were included. Pooled estimates were 92.3\% (90.8-93.7\%) for identification rate, $15.1 \%$ (12.7-17.6\%) for FNR and 36.8\% (34.2-39.5\%) for axillary pCR. After subgroup analysis, FNR is significantly worse if one sentinel node was removed compared to two or more sentinel nodes $(23.9 \%$ versus $10.4 \%, P=0.026)$ and if studies contained clinically nodal stage 1-3, compared to studies with clinically nodal stage 1-2 patients (21.4 versus 13.1\%, $P=0.049$ ). Other factors, including single tracer mapping and the definition of axillary $\mathrm{pCR}$, were not significantly different.

\section{Conclusion}

Based on current evidence it seems not justified to omit further axillary treatment in every clinically node positive breast cancer patients with a negative sentinel lymph node biopsy after neoadjuvant systemic therapy. 


\section{Introduction}

The timing of administration of systemic therapy in breast cancer patients has undergone major changes over the last decade. Chemotherapy and immunotherapy are increasingly administered in neoadjuvant rather than adjuvant setting. ${ }^{1}$ This approach allows in vivo tumour response evaluation to systemic therapy. The downsizing effect of systemic treatment on the primary tumour can facilitate breast conservation therapy where mastectomy would have been indicated initially., ${ }^{2,3}$

The increased use of neoadjuvant systemic therapy creates new clinical dilemmas, especially regarding the nodal staging work-up. In clinically node negative patients it seems appropriate to perform sentinel lymph node biopsy after neoadjuvant systemic therapy. ${ }^{4}$ The current standard for clinically node positive patients is still an axillary lymph node dissection after completion of neoadjuvant therapy. Studies however have reported axillary pathological complete response (pCR) in $20-42 \%$ of initially node positive patients. ${ }^{5-8}$ Therefore performing sentinel lymph node biopsy after neoadjuvant systemic therapy could potentially identify the true node negative patients and prevent unnecessary axillary lymph node dissection. ${ }^{9}$

Many studies have been published on the diagnostic performance of sentinel lymph node biopsy after neoadjuvant systemic therapy in patients with clinically node positive breast cancer, reporting conflicting outcomes regarding the identification rate of a sentinel lymph node and the false-negative rate (FNR) of the procedure.

Our study aim was to investigate the accuracy of sentinel lymph node biopsy in identifying axillary $\mathrm{PCR}$ in pathologically confirmed node positive breast cancer patients receiving neoadjuvant systemic therapy by providing a systematic review and meta-analysis of all relevant studies.

\section{Methods}

\section{Literature search strategy}

For this study PubMed (including Medline) and Embase databases were searched until June 19th, 2015. Free text and Medical Subject Headings (MeSH) terms were used for breast cancer, sentinel lymph node biopsy, diagnostic performance, lymph nodes and neoadjuvant systemic therapy. Full search strategies are provided in Appendix 2.1. 


\section{Study inclusion criteria}

Studies were eligible if accuracy of sentinel lymph node biopsy was evaluated after neoadjuvant systemic therapy in pathologically confirmed node positive breast cancer patients.

Studies had to meet the following inclusion criteria: 1) patients underwent both sentinel lymph node biopsy and axillary lymph node dissection, 2) clinically node positive status was verified by histological examination prior to start neoadjuvant systemic therapy, 3) the nodes retrieved from sentinel lymph node biopsy and axillary lymph node dissection were analyzed by standard institutional guidelines for histological examination, 4) reported data enabled construction of 2 $\mathrm{x} 2$ contingency tables with absolute numbers of true positive (TP), false negative (FN) and true negative (TN) test results.

Only papers written in English, German, French, Spanish or Dutch language were included. Case reports, technical notes, comments on earlier publications, editorials, conference proceedings, reviews or meta-analyses were excluded.

Studies with duplicated results from other studies were excluded. Two independent readers read all abstracts to determine eligibility. Full text articles were read for all potential eligible studies. These readers also decided which studies met the predefined inclusion criteria. In case of discrepancies, consensus was reached by these two readers. Quality assessment of included articles was performed by using the QUADAS-2 tool. ${ }^{10}$

\section{Data extraction}

From studies which met the inclusion criteria, the two independent readers extracted the following predefined parameters: first author, year of publication, study design, sample size, type of primary tumour, clinical tumour and nodal stage, neoadjuvant systemic therapy regimen, the use of immunohistochemistry on axillary nodes, mapping method of sentinel node and definition of pathologic complete axillary response. Data were also extracted on relevant outcome parameters: identification rate, axillary pCR and the false negative rate (FNR) of sentinel lymph node biopsy.

Identification rate was defined as the percentage of patients in which a sentinel node could be identified during surgery divided by all patients in whom a sentinel node procedure was undertaken. Axillary pCR was defined as absence of cancer according to histological diagnosis after axillary lymph node dissection.

Data from patients with successfully identified sentinel nodes were used to construct $2 \times 2$ contingency tables for each study separately. Since false positive cases will not occur, only true positive (TP), false negative (FN) and true negative (TN) values were listed. FNR, the complement of sensitivity, was determined as 
$\mathrm{FN} /(\mathrm{TP}+\mathrm{FN})$ and NPV was determined as TN/(FN + TN $)$. The percentage of patients with axillary $\mathrm{pCR}$ was defined as $\mathrm{TN} /(\mathrm{TP}+\mathrm{FN}+\mathrm{TN})$.

\section{Statistical analysis}

Statistical analyses were performed in STATA 11.2 (StataCorp LP, Texas, USA) and SPSS (version 22, IBM, Armonk, New York, USA).

Random-effects models for meta-analysis were used to calculate pooled proportions for identification rate, FNR and axillary pCR rate with 95\% confidence intervals (CI). ${ }^{11}$ Forest plots show variation in study results and the pooled estimates. Statistical heterogeneity was tested with chi-squared test and was quantified by I2-index. Publication bias was evaluated by funnel plots and by the Egger's test for funnel plot asymmetry. In absence of a relation between sample size and effect size, the study estimates will form a symmetrical funnel shape around the pooled estimate, points from small studies being more dispersed than point from larger studies. Asymmetry indicates an association between sample size and effect size and may be caused by publication bias, but there can also be other causes. ${ }^{12}$

Subgroup analyses were performed to evaluate the impact of several factors on identification rate and FNR. Factors that were considered relevant were year of publication, study design, sample size, clinical nodal stage at presentation, definition of axillary pCR and additional immunohistochemistry. Linear regression analyses were used to correlate FNR with identification rate and FNR with axillary pCR. Statistical significance was considered as $P$-values (two-sided) $<0.05$.

\section{Results}

\section{Search strategy and study selection}

The literature search identified 1146 publications. After removing 149 duplicates, the reading of 997 abstracts of the remaining papers resulted in the selection of 28 eligible papers. Full text of all these papers was read. Finally, eight articles were considered to meet the inclusion criteria and were included for final review (Figures 2.1 and 2.2).

\section{General study characteristics}

In these eight different studies, a total of 1395 patients participated (range 61687). ${ }^{13-20}$ Initial node positive disease was either cytologically or histologically proven for all patients at initial diagnosis. Neoadjuvant systemic therapy regimens 
varied within studies. Sentinel lymph node procedure was followed by complete axillary lymph node dissection in all studies. Table 2.1 shows a detailed overview of all study characteristics. Table 2.2 shows an overview of all contingency tables with absolute numbers of the selected studies (including the NPVs of all studies).

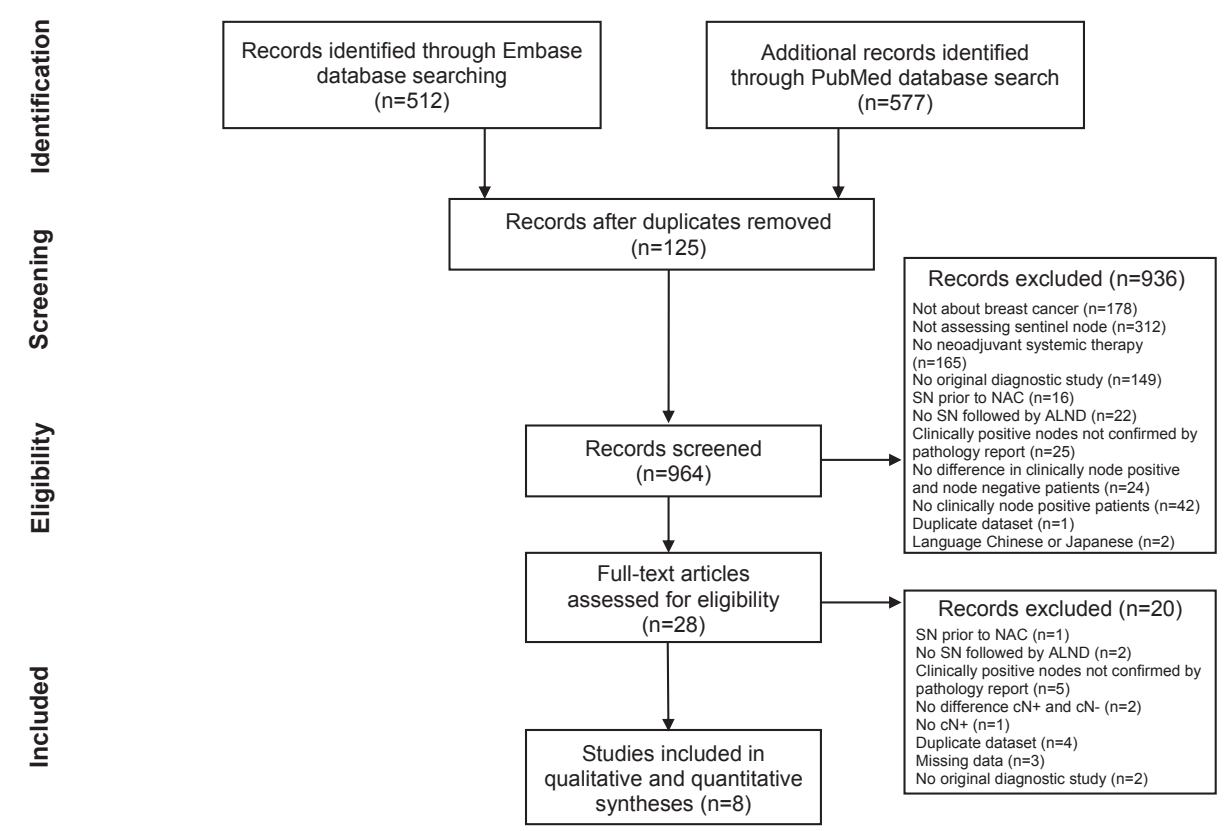

Figure 2.1 Flow diagram of preferred reporting items for systematic reviews and meta-analyses (PRISMA).

\begin{tabular}{|c|c|c|c|c|c|c|c|}
\hline \multirow[t]{2}{*}{ Study } & \multicolumn{4}{|c|}{ Risk of bias } & \multicolumn{3}{|c|}{ Applicability concerns } \\
\hline & $\begin{array}{l}\text { Patient } \\
\text { selection }\end{array}$ & Index test & $\begin{array}{l}\text { Reference } \\
\text { standard }\end{array}$ & $\begin{array}{c}\text { Flow and } \\
\text { timing }\end{array}$ & $\begin{array}{c}\text { Patient } \\
\text { selection }\end{array}$ & Index test & $\begin{array}{l}\text { Reference } \\
\text { standard }\end{array}$ \\
\hline Boileau & (:) & (-) & (:) & (;) & (;) & (:) & () \\
\hline Brown & (2) & (:) & (:) & (:) & $?$ & (:) & (-) \\
\hline Kang & (-) & (:) & (:) & (2) & (:) & (:) & (:) \\
\hline Ozmen & (:) & (:) & (:) & (:) & (:) & (:) & (:) \\
\hline Yagata & (:) & (:) & (:) & (:) & (2) & (:) & (:) \\
\hline
\end{tabular}

(:) = Low risk, $\odot=$ High risk, ? = Unclear risk

Figure 2.2 QUADAS-2 tool. Evaluation of risk of bias and applicability of primary diagnostic accuracy studies. 


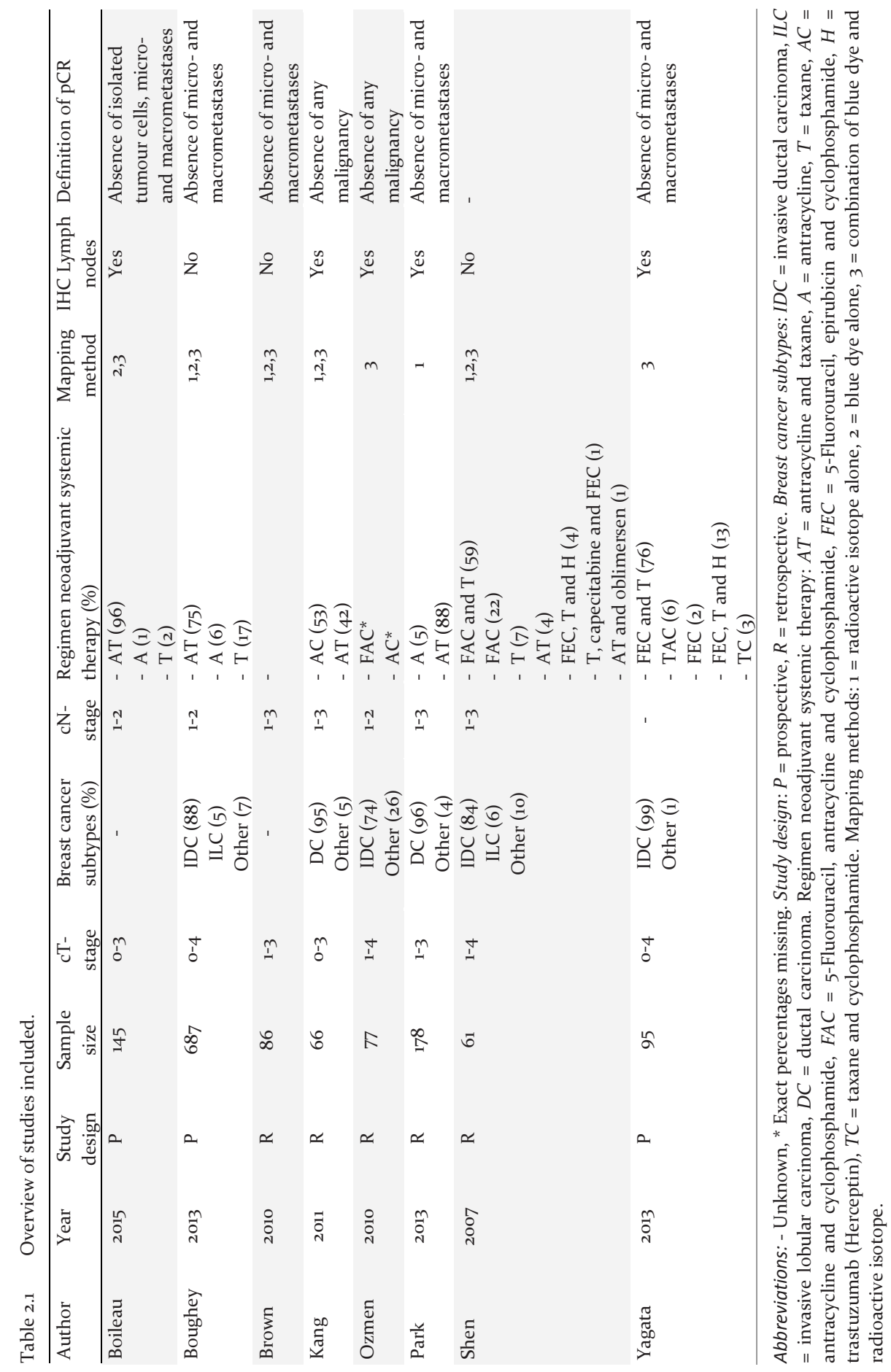




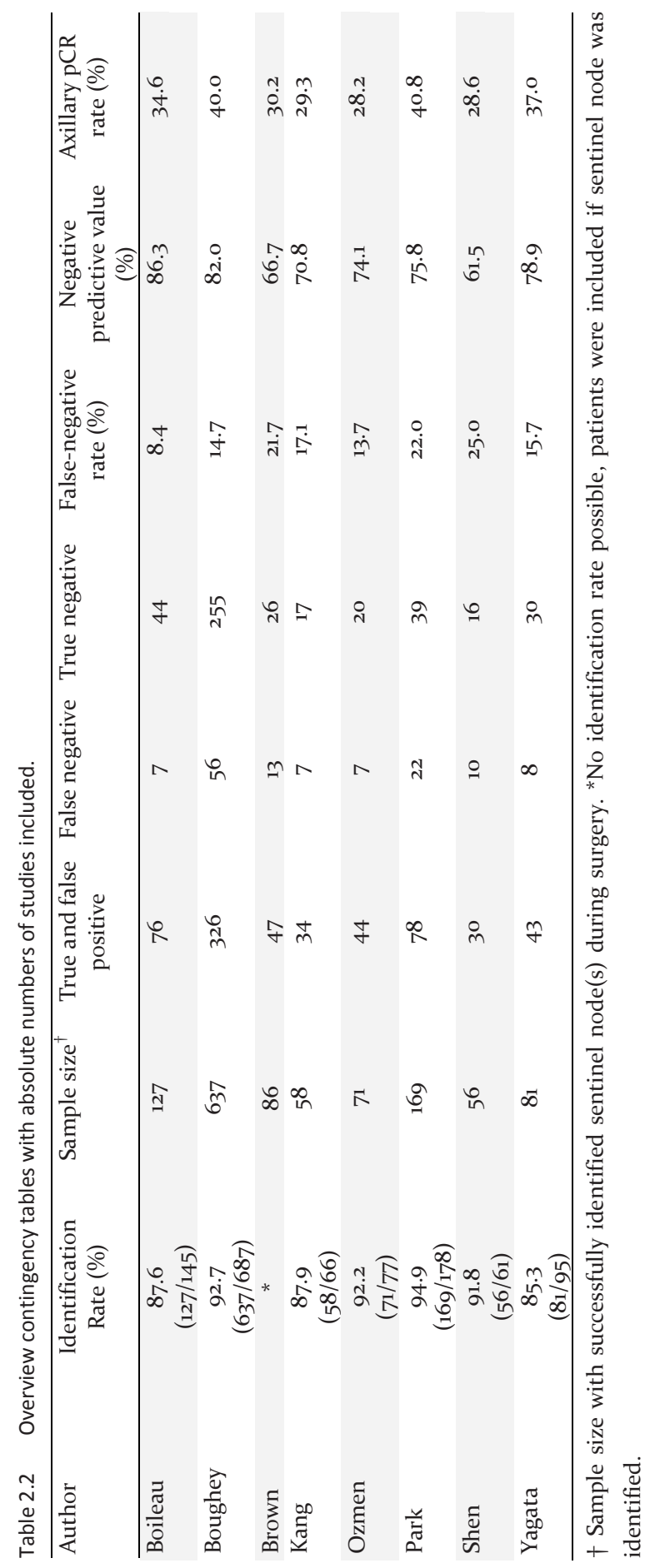




\section{Sentinel lymph node biopsy}

\section{Identification rate}

One of the included studies could not be used for the assessment of identification rate, because it was not reported. ${ }^{15}$ Figure 2.3 shows the Forest plot and funnel plot for study results. The pooled identification rate was 92.3\% (95\% CI, 90.8-93.7\%). The funnel plot indicates a lower identification rate in studies with small sample size. The Egger's test resulted in an intercept of 1 and $P=0.120$.

Four studies subdivided their populations according to their different mapping methods for the identification of the sentinel lymph node during surgery: radioactive isotope alone, blue dye alone and the combination of both

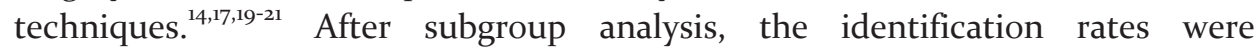
respectively $93.9 \%(95 \% \mathrm{CI}, 91.2-96.7 \%)$ for radioactive isotope alone, $78.8 \%$ (95\% CI, 64.9-92.7\%) for blue dye alone and $93.0 \%$ (95\% CI, 91.2-94.8\%) for the combination of both techniques. There were no statistical significances between blue dye and radioactive isotope alone $(P=0.18)$, and blue dye and the combination of both techniques $(P=0.151)$.

Further subgroup analyses showed no significant difference for year of publication, study design, sample size and clinical nodal stage (Appendix 2.2).

A

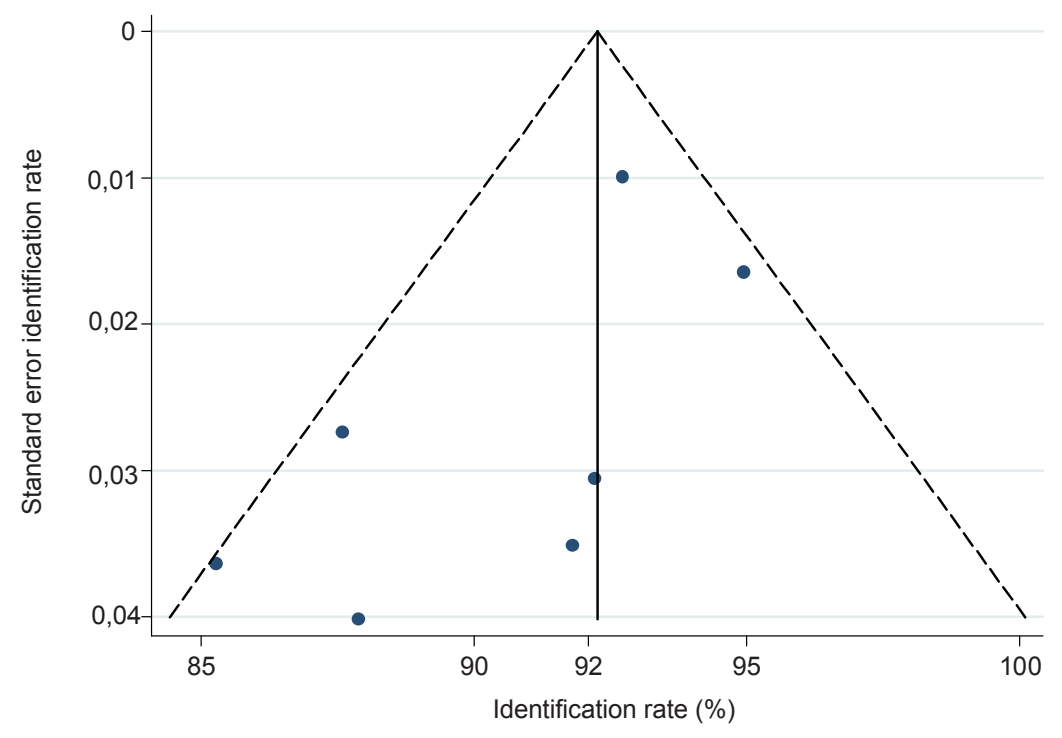


B

\begin{tabular}{|c|c|c|}
\hline Study (year) & Effect Size $(95 \%$ Cl) & Weight (\%) \\
\hline Boileau (2015) & $0.88(0.82,0.93)$ & 7.22 \\
\hline Boughey (2013) & $0.93(0.91,0.95)$ & 55.09 \\
\hline Kang (2011) & $0.88(0.80,0.96)$ & 3.35 \\
\hline Ozmen (2010) & $0.92(0.86,0.98)$ & 5.80 \\
\hline Park (2013) & $\rightarrow 0.95(0.92,0.98)$ & 20.06 \\
\hline Shen (2007) & $0.92(0.85,0.99)$ & 4.39 \\
\hline Yagata (2013) & $0.85(0.78,0.92)$ & 4.09 \\
\hline Overall (I-squared $=44.0 \%, p=0.098)$ & $0.92(0.91,0.94)$ & 100.00 \\
\hline
\end{tabular}

Figure 2.3 Forest plot (A) and funnel plot (B) with pseudo $95 \%$ confidence intervals of identification rate. The pooled identification rate was $92.3 \%\left(I^{2}=44.0 \%, P=0.098\right)$.

\section{False-negative rate}

Figure 2.4 shows the Forest plot and funnel plot for study results. The pooled FNR was $15.1 \%$ (95\% CI, 12.7-17.6\%) with an $\mathrm{I}^{2}$ index of $38.8 \%$. The funnel plot for FNR shows that studies with small sample size tended to report higher FNRs than studies with larger sample size. The Egger's test resulted in an intercept of 1 and $P=0.224$. Figure 2.5 shows the correlation between FNR and identification rate of the included studies $(P=0.290)$. There was no correlation between FNR and axillary pCR $(P=0.714)$.

Two of the included studies used non-invasive restaging after neoadjuvant systemic therapy to identify clinically No patients after neoadjuvant systemic therapy, with the objective to perform the sentinel lymph node biopsy only in clinically No patients. ${ }^{17,20}$ Restaging consisted of physical examination, mammography, ultrasound or breast MRI. According to subgroup analysis, the FNR of these two studies was $14.7 \%$ (95\% CI 7.8-21.5\%), which was comparable to FNR of $15.2 \%$ (95\% CI 12.6-17.8\%) for all other studies $(P=0.885)$.

Four studies subdivided their populations in patients with only one sentinel node removed during surgery versus patients with two or more sentinel nodes removed during surgery. ${ }^{13,14,18,20}$ After subgroup analysis in these studies, FNR was significantly higher if only one sentinel node was removed compared to two or more sentinel nodes with a pooled FNR of $23.9 \%$ (95\% CI 17.2-30.6\%) versus $10.4 \%$ $(95 \%$ CI 7.7-13.2\%) $(P=0.026)$. 
Further subgroup analysis showed a significantly higher FNR for studies which included patients with clinically nodal stage 1-3, compared to studies which only contained patients with clinically nodal stage $1-2$ (21.4 versus $13.1 \%, P=0.049$ ). Moreover, a prospective study design showed a trend towards significant improvement of FNR (13.3 versus 19.6\%, $P=0.079$, respectively) (Appendix 2.2).

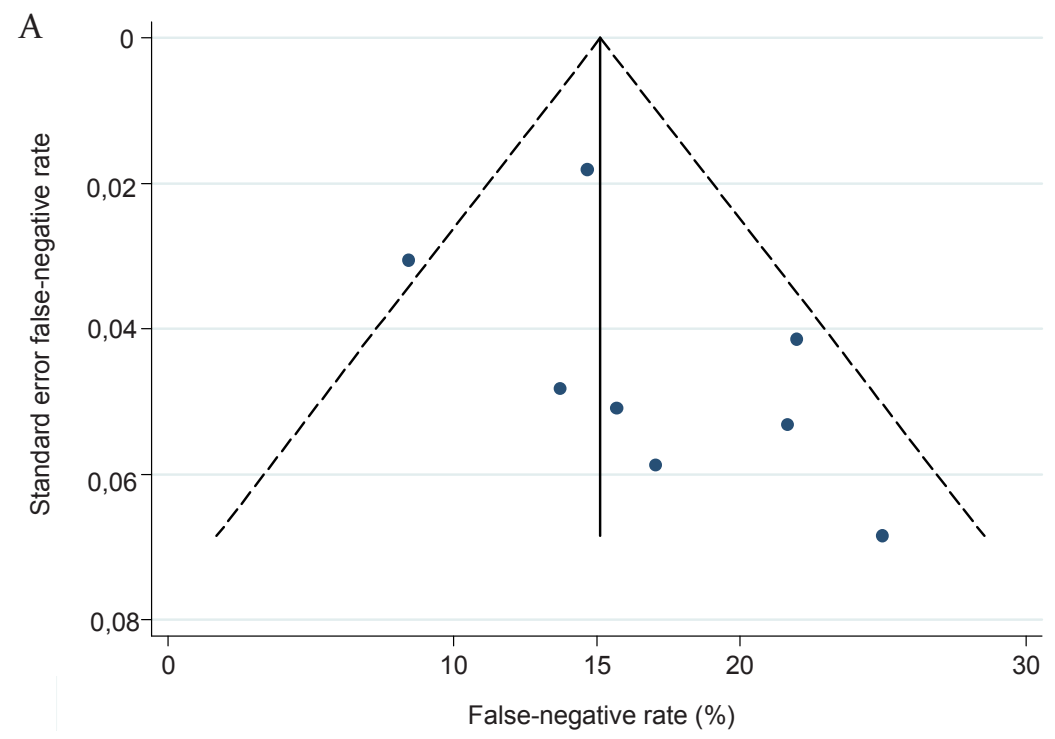

B

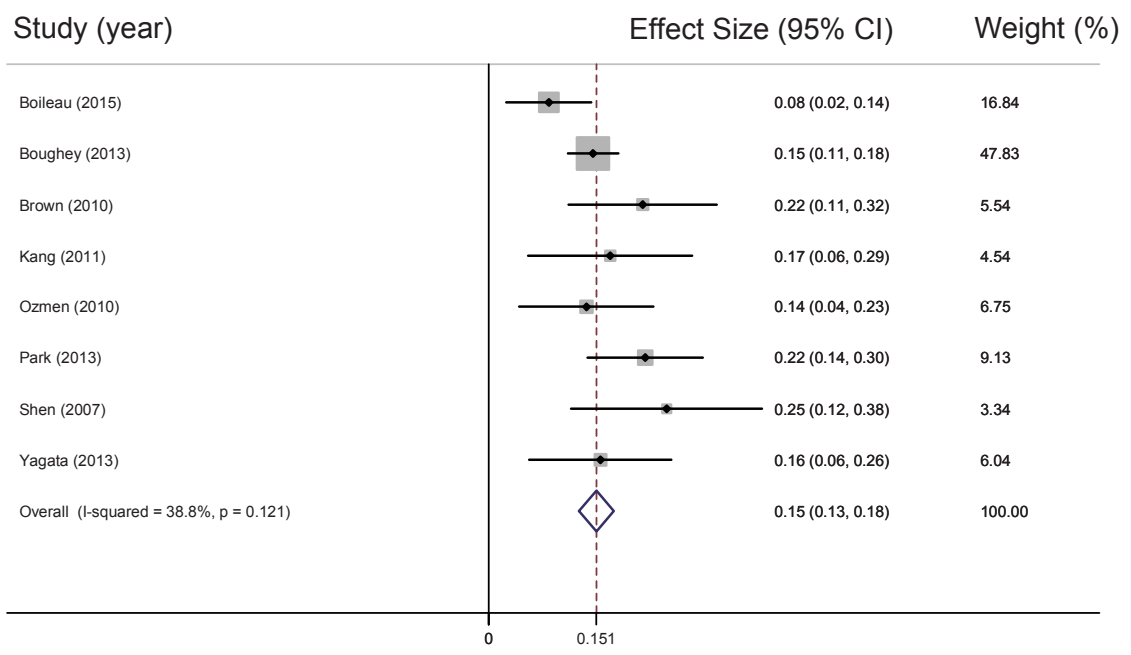

Figure 2.4 Forest plot (A) and funnel plot (B) with pseudo 95\% confidence intervals of false-negative rate. The pooled false-negative rate was $15 \cdot 1 \%\left(I^{2}=38.8 \%, P=0.120\right)$. 


\section{Response evaluation}

\section{Axillary pathological complete response}

In absolute numbers, 477 out of 1395 initially node positive patients achieved axillary pCR to neoadjuvant systemic therapy. Appendix 2.3 shows the Forest plot and funnel plot for study results. The pooled axillary pCR rate was $36.8 \%$ (95\% CI, $34.2-39.5 \%$ ) with an $\mathrm{I}^{2}$ index of $41.5 \%$. The funnel plot for axillary $\mathrm{pCR}$ shows that studies with small sample size tended to report lower pCRs than studies with larger sample size. The Egger's test resulted in an intercept of 1 and $P=0.102$.

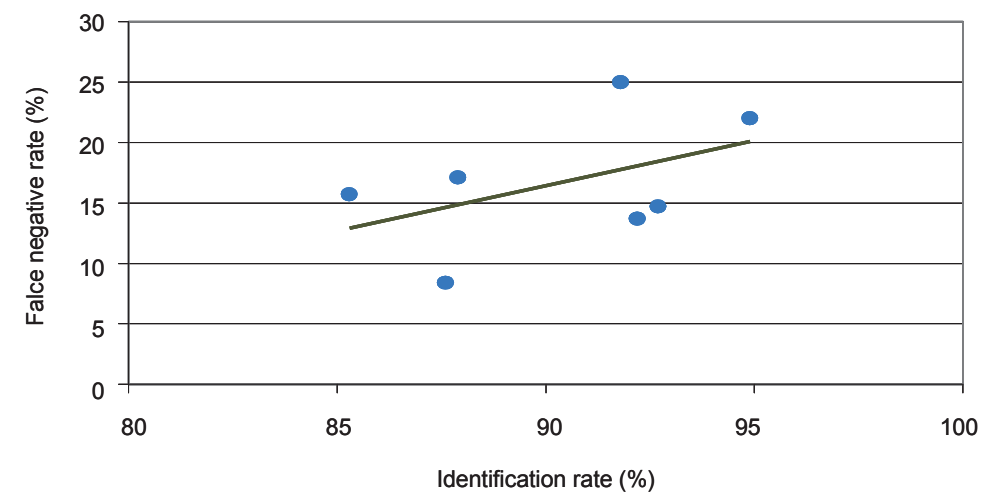

Figure 2.5 False-negative rate as function of identification rate. Increase of false-negative rate is associated with an increase of identification rate $(P=0.290)$.

\section{Discussion}

This meta-analysis provides an overview of the diagnostic performance of the sentinel lymph node biopsy after neoadjuvant systemic therapy in pathologically confirmed initially node positive breast cancer patients. Our study showed a pooled identification rate of $92.3 \%$ and a pooled FNR of $15.1 \%$.

Axillary lymph node dissection is currently the standard practice in clinically node positive breast cancer patients. ${ }^{5}$ It is associated with severe side effects in $45 \%$ of its patients, such as lymphedema, neuropathy and functional limitations. ${ }^{22}$ Clinically node positive patients converting to node negative status after neoadjuvant systemic therapy could potentially benefit from omitting an axillary lymph node dissection thus increasing the quality of life of breast cancer survivors. A prerequisite is a diagnostic test that can accurately select the patients with axillary pCR. 
The success of identifying the sentinel lymph node during surgery in clinically node negative patients is inferior after neoadjuvant systemic therapy compared to primary surgery. ${ }^{23}$ Despite the use of dual tracer mapping as opposed to single tracer mapping in sentinel lymph node biopsy, identification rate in clinically node negative patients remains inferior (95 versus 98\%). ${ }^{24}$ We hypothesize that both the presence of axillary metastases and the effect of the systemic treatment may contribute to changes in the lymphatic flow. ${ }^{21}$ For this meta-analysis, the use of blue dye alone as single tracer mapping agent showed a lower identification rate, compared to radioactive isotope alone or the combination of both techniques (78.8\% versus 93.3 and $93.0 \%$ ), however it was not statistically significant due to the small sample size of patients who received blue dye alone for tracer mapping ( $\mathrm{n}=33$ for blue dye alone, compared to $\mathrm{n}=301$ for radioactive isotope alone and $\mathrm{n}=774$ for dual tracer mapping).

According to the studies of Harlow et al. ${ }^{25}$ and Kim et al., ${ }^{26}$ sentinel lymph node biopsy with a FNR of $10 \%$ is accepted as oncologically safe to avoid axillary lymph node dissection in clinically node negative patients undergoing primary surgery. Based on these results, recent studies in clinically node positive patients treated with neoadjuvant systemic therapy used a cut-off value of $10 \%$ for FNR. ${ }^{13,14}$ The use of this cut-off value of $10 \%$ is arbitrary. Maybe a FNR of $5 \%$ or even $15 \%$ would be more appropriate. Patients with a false negative sentinel lymph node biopsy are theoretically at risk to develop regional (nodal) recurrences, with reduced disease-free and overall survival. However, omission of axillary lymph node removal in clinically node negative patients does not affect survival according to the ACOSOG Zooil trial. ${ }^{27}$ In clinically node positive patients undergoing neoadjuvant systemic therapy, we assume patients will still receive adjuvant trastuzumab, radiotherapy and/or endocrine therapy on indication. Obviously, all patients already received appropriate chemotherapy. For these patients, the role of regional axillary radiotherapy to prevent regional recurrences will be investigated in the NSABP B-51 trial and the Alliance 11202 trial, both registered with Clinicaltrials.gov (NCTo1872975 and NCTo1901094). These trials will give us the final information on the safety of the omission of further axillary treatment in clinically node positive patients with a negative sentinel node after neoadjuvant systemic therapy.

We observed that FNR is mainly influenced by four conditions in clinically node positive or negative sentinel lymph node studies. First, FNR in clinically node negative patients is worse in whom only one sentinel node could be identified during surgery, compared to two or more sentinel nodes ( $14.3 \%$ versus $4.3 \%) .^{28}$ In this meta-analysis four studies investigated the difference between one and two or more sentinel nodes removed per patient. Subgroup analysis reported a higher FNR if one instead of two or more sentinel nodes were removed during 
surgery $\left(23.9 \%\right.$ versus $10.7 \%, P=0.026$, respectively). ${ }^{14,18}$ Although this finding seems promising, in clinical practice it is impossible to predict the number of sentinel nodes identified per patient during surgery. So far, no studies have investigated the value of additional node picking in case only one or two sentinel nodes are identified.

Second, extensive evaluation of the sentinel lymph node using immunohistochemistry seemed to reduce the FNR, from 13.3 to $8.4 \% .^{13}$ However, it is unclear in how many patients the sentinel lymph node contained isolated tumour cells, while the complementary axillary lymph node dissection revealed no additional metastases, thereby introducing possible overtreatment. ${ }^{13}$ Since, the importance of the detection of isolated tumour cells as indicator to decide whether or not to perform a complementary axillary lymph node dissection is unclear.

Third, FNR seems to be associated with the identification rate $(P=0.290)$ (Figure 2.5). One would expect a reduction of FNR when the identification rate increases, suggesting performance of a more experienced surgeon during the sentinel lymph node biopsy. ${ }^{29}$ However, according to our meta-analysis this is questionable.

Finally, the number of patients converting to axillary $\mathrm{pCR}$ in initially node positive patients can influence the FNR. If the amount of true negative patients reduces, for instance in low grade hormone-receptor positive, HER2-negative breast cancer patients treated with neoadjuvant systemic therapy with a reported pCR of $7.5 \%$ for both primary tumour and axilla, ${ }^{30}$ both FNR and additionally NPV could reduce. However, for this meta-analysis there was no correlation between FNR and axillary pCR $(P=0.714)$.

The sentinel lymph node procedure for axillary staging seems inappropriate in patients with clinically nodal stage 3 . Our results showed significantly higher FNR in these cases (21.4 versus 13.1\%, $P=0.049$ ). Periclavicular malignant lymph nodes are not always associated with presence of axillary lymph node metastases. ${ }^{31,32}$ Consequently, FNR can increase since metastases may be located elsewhere.

Noninvasive nodal restaging after neoadjuvant systemic therapy in clinically node positive patients with breast cancer prior to sentinel lymph node biopsy could be of additional value. ${ }^{33}$ In our meta-analysis, two studies included only pathologically confirmed node positive patients who converted to clinically node negative after neoadjuvant systemic therapy. ${ }^{17,20}$ In these studies physical examination, ultrasound or MRI was used for nodal restaging. Nonetheless the pooled FNR for these two studies was not lower than the pooled FNR in the other studies ( 14.7 versus $15.2 \%, P=0.885$ ). For these two studies it might be possible that 
many true positive patients were excluded disproportionate to few false negative patients.

One of the inclusion criterion for this meta-analysis was cytological or histopathological confirmation of nodal malignancy at diagnosis, since node positivity based on clinical imaging and examination results in inaccurate nodal staging. ${ }^{34-36}$ Recently, Feng et al. demonstrated the importance of pathological confirmation for axillary nodal staging. ${ }^{37}$ In their study, the PPV for axillary ultrasound in breast cancer patients improved from 79.6 towards $100 \%$, when fine-needle cytology was added to the general axillary ultrasound. As a consequence, several studies were excluded for this meta-analysis due to differences in defining clinically node positive patients, like the SENTINA trial. ${ }^{38-40}$ In the SENTINA trial, pathologically confirmed nodal disease was not required for inclusion, resulting in the definition of node positive disease based on ultrasound. Including these studies for the meta-analysis may have led to overestimation of the results, as the axillary $\mathrm{pCR}$ rate is higher in these studies (40.o versus $52.3 \%) .{ }^{14,38}$

Our meta-analysis suggests that the sentinel node procedure after systemic treatment of a clinically node positive breast cancer patient is insufficiently accurate to identify the patient with axillary pCR. Other, rather noninvasive techniques for nodal restaging are warranted. In imaging, PET-CT ${ }^{41,42}$ and lymph node specific contrast-enhanced axillary $\mathrm{MRI}^{43,44}$ still need further exploration in order to prevent patients from invasive procedures for nodal staging, with reported PPV and NPV of 67 and $56 \%$ for PET-CT, ${ }^{45} 75 \%$ and $43 \%$ for MRI, respectively. ${ }^{46}$ Furthermore, marking malignant lymph nodes with radioactive iodine seeds is promising. Donker et al. achieved a FNR as low as $7 \%$ despite the fact that one third of the study patients had clinically nodal stage $3 \cdot{ }^{47}$ Yet the NPV of $83.3 \%$ is still insufficiently accurate to decide based on the MARI procedure that axillary surgery can be safely omitted. ${ }^{48}$

As an alternative, a targeted axillary dissection can be performed. This procedure consists of a sentinel lymph node biopsy and removal of a clip containing previous positive node for the evaluation of $\mathrm{pCR}$ in clinically node positive breast cancer patients treated with neoadjuvant systemic therapy. ${ }^{49}$ The clip-containing lymph node was not always in line with the sentinel node (1 out of 5 patients), indicating this technique might become a more appropriate tool for predicting $\mathrm{PCR}$ in future, as targeted axillary dissection could possibly identify more often the malignant node compared to the sentinel lymph node biopsy.

In conclusion, based on current evidence it seems not justified to omit further axillary treatment in every clinically node positive breast cancer patient with a negative sentinel lymph node biopsy after neoadjuvant systemic therapy. 


\section{References}

1. Kaufmann M, von Minckwitz G, Mamounas EP, et al. Recommendations from an international consensus conference on the current status and future of neoadjuvant systemic therapy in primary breast cancer. Ann Surg Oncol 2012;19:1508-16.

2. Fisher B, Bryant J, Wolmark N, et al. Effect of preoperative chemotherapy on the outcome of women with operable breast cancer. J Clin Oncol 1998;16:2672-85.

3. van der Hage JA, van de Velde CJ, Julien JP, Tubiana-Hulin M, Vandervelden C, Duchateau L. Preoperative chemotherapy in primary operable breast cancer: results from the European Organization for Research and Treatment of Cancer trial 10902. J Clin Oncol 2001;19:4224-37.

4. Krag DN, Anderson SJ, Julian TB, et al. Sentinel-lymph-node resection compared with conventional axillary-lymph-node dissection in clinically node-negative patients with breast cancer: overall survival findings from the NSABP B-32 randomised phase 3 trial. Lancet Oncol 2010;11:927-33.

5. Alvarado R, Yi M, Le-Petross $\mathrm{H}$, et al. The role for sentinel lymph node dissection after neoadjuvant chemotherapy in patients who present with node-positive breast cancer. Ann Surg Oncol 2012;19:3177-84.

6. Koolen BB, Valdes Olmos RA, Wesseling J, et al. Early assessment of axillary response with (1)(8)F-FDG PET/CT during neoadjuvant chemotherapy in stage II-III breast cancer: implications for surgical management of the axilla. Ann Surg Oncol 2013;20:2227-35.

7. Rouzier R, Extra JM, Klijanienko J, et al. Incidence and prognostic significance of complete axillary downstaging after primary chemotherapy in breast cancer patients with $\mathrm{T}_{1}$ to $\mathrm{T}_{3}$ tumors and cytologically proven axillary metastatic lymph nodes. J Clin Oncol 2002;20:1304-10.

8. Straver ME, Rutgers EJ, Russell NS, et al. Towards rational axillary treatment in relation to neoadjuvant therapy in breast cancer. Eur J Cancer 2009;45:2284-92.

9. Fontein DB, van de Water W, Mieog JS, Liefers GJ, van de Velde CJ. Timing of the sentinel lymph node biopsy in breast cancer patients receiving neoadjuvant therapy e recommendations for clinical guidance. Eur J Surg Oncol 2013;39:417-24.

10. Whiting PF, Rutjes AW, Westwood ME, et al. QUADAS-2: a revised tool for the quality assessment of diagnostic accuracy studies. Ann Intern Med 2011;155:529-36.

11. DerSimonian R, Laird N. Meta-analysis in clinical trials. Control Clin Trials 1986;7:177-88.

12. Egger M, Davey Smith G, Schneider M, Minder C. Bias in metaanalysis detected by a simple, graphical test. BMJ 1997;315:629-34.

13. Boileau JF, Poirier B, Basik M, et al. Sentinel node biopsy after neoadjuvant chemotherapy in biopsy-proven node-positive breast cancer: the SN FNAC study. J Clin Oncol 2015;33:258-64.

14. Boughey JC, Suman VJ, Mittendorf EA, et al. Alliance for Clinical Trials in O. Sentinel lymph node surgery after neoadjuvant chemotherapy in patients with node-positive breast cancer: the ACOSOG Z1071 (Alliance) clinical trial. JAMA 2013;310:1455-61.

15. Brown AS, Hunt KK, Shen J, et al. Histologic changes associated with false-negative sentinel lymph nodes after preoperative chemotherapy in patients with confirmed lymph node-positive breast cancer before treatment. Cancer 2010;116:2878-83.

16. Kang E, Chung IY, Han SA, et al. Feasibility of sentinel lymph node biopsy in breast cancer patients with initial axillary lymph node metastasis after primary systemic therapy. J Breast Cancer 2011;14:147-52.

17. Ozmen V, Unal ES, Muslumanoglu ME, et al. Axillary sentinel node biopsy after neoadjuvant chemotherapy. Eur J Surg Oncol 2010;36:23-9.

18. Park S, Park JM, Cho JH, Park HS, Kim SI, Park BW. Sentinel lymph node biopsy after neoadjuvant chemotherapy in patients with cytologically proven node-positive breast cancer at diagnosis. Ann Surg Oncol 2013;20:2858-65.

19. Shen J, Gilcrease MZ, Babiera GV, et al. Feasibility and accuracy of sentinel lymph node biopsy after preoperative chemotherapy in breast cancer patients with documented axillary metastases. Cancer 2007;109:1255-63. 
2o. Yagata H, Yamauchi H, Tsugawa K, et al. Sentinel node biopsy after neoadjuvant chemotherapy in cytologically proven node-positive breast cancer. Clin Breast Cancer 2013;13:471-7.

21. Boughey JC, Suman VJ, Mittendorf EA, et al. Alliance for Clinical Trials in O. Factors affecting Sentinel lymph node identification rate after neoadjuvant chemotherapy for breast Cancer patients Enrolled in ACOSOG Z1071 (Alliance). Ann Surg 2015;261:547-52.

22. Ahmed RL, Prizment A, Lazovich D, Schmitz KH, Folsom AR. Lymphedema and quality of life in breast cancer survivors: the Iowa Women's Health Study. J Clin Oncol 2008;26:5689-96.

23. Xing Y, Foy M, Cox DD, Kuerer HM, Hunt KK, Cormier JN. Metaanalysis of sentinel lymph node biopsy after preoperative chemotherapy in patients with breast cancer. Br J Surg 2006;93:539-46.

24. van der Heiden-van der Loo M, de Munck L, Sonke GS, et al. Population based study on sentinel node biopsy before or after neoadjuvant chemotherapy in clinically node negative breast cancer patients: identification rate and influence on axillary treatment. Eur J Cancer 2015;51:915-21.

25. Harlow SP, Krag DN, Julian TB, et al. Prerandomization surgical training for the National Surgical Adjuvant Breast and Bowel Project (NSABP) B-32 trial: a randomized phase III clinical trial to compare sentinel node resection to conventional axillary dissection in clinically nodenegative breast cancer. Ann Surg 2005;241:48-54.

26. Kim T, Giuliano AE, Lyman GH. Lymphatic mapping and sentinel lymph node biopsy in earlystage breast carcinoma: a metaanalysis. Cancer 2006;106:4-16.

27. Giuliano AE, Hunt KK, Ballman KV, et al. Axillary dissection vs no axillary dissection in women with invasive breast cancer and sentinel node metastasis: a randomized clinical trial. JAMA 2011;305:569-75.

28. Wong SL, Edwards MJ, Chao C, et al. Sentinel lymph node biopsy for breast cancer: impact of the number of sentinel nodes removed on the false-negative rate. J Am Coll Surg 2001;192:684-9. discussion geg1.

29. Bernardi S, Bertozzi S, Londero AP, Angione V, Petri R, Giacomuzzi F. Prevalence and risk factors of intraoperative identification failure of sentinel lymph nodes in patients affected by breast cancer. Nucl Med Commun 2013;34:664-73.

30. Cortazar P, Zhang L, Untch M, et al. Pathological complete response and long-term clinical benefit in breast cancer: the CTNeoBC pooled analysis. Lancet 2014;384:164-72.

31. Huang EH, Strom EA, Valero V, et al. Locoregional treatment outcomes for breast cancer patients with ipsilateral supraclavicular metastases at diagnosis. Int J Radiat Oncol Biol Phys 2007;67:490-6.

32. Zhang YJ, Oh JL, Whitman GJ, et al. Clinically apparent internal mammary nodal metastasis in patients with advanced breast cancer: incidence and local control. Int J Radiat Oncol Biol Phys 2010;77:1113-9.

33. Boughey JC, Ballman KV, Hunt KK, et al. Axillary ultrasound after neoadjuvant chemotherapy and its impact on Sentinel lymph node surgery: results from the American College of Surgeons Oncology Group Z1071 Trial (Alliance). J Clin Oncol 2015;33:3386-93.

34. Alvarez S, Anorbe E, Alcorta P, Lopez F, Alonso I, Cortes J. Role of sonography in the diagnosis of axillary lymph node metastases in breast cancer: a systematic review. AJR Am J Roentgenol 2006;186:1342-8.

35. Diepstraten SC, Sever AR, Buckens CF, et al. Value of preoperative ultrasound-guided axillary lymph node biopsy for preventing completion axillary lymph node dissection in breast cancer: a systematic review and meta-analysis. Ann Surg Oncol 2014;21:51-9.

36. Valente SA, Levine GM, Silverstein MJ, et al. Accuracy of predicting axillary lymph node positivity by physical examination, mammography, ultrasonography, and magnetic resonance imaging. Ann Surg Oncol 2012;19:1825-30.

37. Feng Y, Huang R, He Y, et al. Efficacy of physical examination, ultrasound, and ultrasound combined with fine-needle aspiration for axilla staging of primary breast cancer. Breast Cancer Res Treat 2015;149:761-5.

38. Kuehn T, Bauerfeind I, Fehm T, et al. Sentinel-lymph-node biopsy in patients with breast cancer before and after neoadjuvant chemotherapy (SENTINA): a prospective, multicentre cohort study. Lancet Oncol 2013;14:609-18. 
39. Classe JM, Bordes V, Campion L, et al. Sentinel lymph node biopsy after neoadjuvant chemotherapy for advanced breast cancer: results of Ganglion Sentinelle et Chimiotherapie Neoadjuvante, a French prospective multicentric study. J Clin Oncol 2009;27:726-32.

40. Shigekawa T, Sugitani I, Takeuchi H, et al. Axillary ultrasound examination is useful for selecting patients optimally suited for sentinel lymph node biopsy after primary systemic chemotherapy. Am J Surg 2012;204:487-93.

41. Koolen BB, Valdes Olmos RA, Elkhuizen PH, et al. Locoregional lymph node involvement on 18FFDG PET/CT in breast cancer patients scheduled for neoadjuvant chemotherapy. Breast Cancer Res Treat 2012;135:231-40.

42. Krammer J, Schnitzer A, Kaiser CG, et al. F-FDG PET/CT for initial staging in breast cancer patients - is there a relevant impact on treatment planning compared to conventional stagingmodalities? Eur Radiol 2015;25:2460-9.

43. Schipper RJ, Smidt ML, van Roozendaal LM, et al. Noninvasive nodal staging in patients with breast cancer using gadofosveset-enhanced magnetic resonance imaging: a feasibility study. Invest Radiol 2013;48:134-9.

44. Kuijs VJ, Moossdorff M, Schipper RJ, et al. The role of MRI in axillary lymph node imaging in breast cancer patients: a systematic review. Insights Imaging 2015;6:203-15.

45. Vicente AMG, Castrejon AS, Martin AL, et al. Early and delayed prediction of axillary lymph node neoadjuvant response by F-18-FDG PET/CT in patients with locally advanced breast cancer. Eur J Nucl Med Mol Imaging 2014;41:1309-18.

46. Hieken TJ, Boughey JC, Jones KN, Shah SS, Glazebrook KN. Imaging response and residual metastatic axillary lymph node disease after neoadjuvant chemotherapy for primary breast cancer. Ann Surg Oncol 2013;20:3199-204.

47. Donker M, Straver ME, Wesseling J, et al. Marking axillary lymph nodes with radioactive iodine seeds for axillary staging after neoadjuvant systemic treatment in breast cancer patients: the MARI procedure. Ann Surg 2015;261:378-82.

48. Vugts G, Schipper RJ, Maaskant-Braat AJ, Smidt ML, Nieuwenhuijzen GA. Axillary response monitoring after neoadjuvant chemotherapy in breast cancer: can we avoid the morbidity of axillary treatment? Ann Surg 2016;263:e28-9

49. Caudle AS, Yang WT, Mittendorf EA, et al. Selective surgical localization of axillary lymph nodes containing metastases in patients with breast cancer: a prospective feasibility trial. JAMA Surg 2015;150:137-43. 


\section{Appendix 2.1}

\section{Search strategy for systematic review and meta-analysis of sentinel lymph node biopsy in clinical node positive patients receiving neoadjuvant systemic therapy}

\section{Embase search strategy}

1. breast.mp. [mp=title, abstract, subject headings, heading word, drug trade name, original title, device manufacturer, drug manufacturer, device trade name, keyword]

2. mamma.mp. [mp=title, abstract, subject headings, heading word, drug trade name, original title, device manufacturer, drug manufacturer, device trade name, keyword]

3. 1 or 2

4. cancer.mp. [mp=title, abstract, subject headings, heading word, drug trade name, original title, device manufacturer, drug manufacturer, device trade name, keyword]

5. carcinoma.mp. [mp=title, abstract, subject headings, heading word, drug trade name, original title, device manufacturer, drug manufacturer, device trade name, keyword]

6. neoplasm.mp. [mp=title, abstract, subject headings, heading word, drug trade name, original title, device manufacturer, drug manufacturer, device trade name, keyword]

7. malignancy.mp. [mp=title, abstract, subject headings, heading word, drug trade name, original title, device manufacturer, drug manufacturer, device trade name, keyword]

8. adenocarcinoma.mp. [mp=title, abstract, subject headings, heading word, drug trade name, original title, device manufacturer, drug manufacturer, device trade name, keyword]

9. 4 or 5 or 6 or 7 or 8

10. 3 and 9

11. sentinel lymph node biopsy.mp. [mp=title, abstract, subject headings, heading word, drug trade name, original title, device manufacturer, drug manufacturer, device trade name, keyword]

12. slnb.mp. [mp=title, abstract, subject headings, heading word, drug trade name, original title, device manufacturer, drug manufacturer, device trade name, keyword]

13. sentinel node.mp. [mp=title, abstract, subject headings, heading word, drug trade name, original title, device manufacturer, drug manufacturer, device trade name, keyword] 


\section{11 or 12 or 13}

15. sensitivity.mp. [mp=title, abstract, subject headings, heading word, drug trade name, original title, device manufacturer, drug manufacturer, device trade name, keyword]

16. specificity.mp. [mp=title, abstract, subject headings, heading word, drug trade name, original title, device manufacturer, drug manufacturer, device trade name, keyword]

17. predictive value.mp. [mp=title, abstract, subject headings, heading word, drug trade name, original title, device manufacturer, drug manufacturer, device trade name, keyword]

18. positive predictive value.mp. [mp=title, abstract, subject headings, heading word, drug trade name, original title, device manufacturer, drug manufacturer, device trade name, keyword]

19. negative predictive value.mp. [mp=title, abstract, subject headings, heading word, drug trade name, original title, device manufacturer, drug manufacturer, device trade name, keyword]

2o. likelihood ratio.mp. [mp=title, abstract, subject headings, heading word, drug trade name, original title, device manufacturer, drug manufacturer, device trade name, keyword]

21. ppv.mp. [mp=title, abstract, subject headings, heading word, drug trade name, original title, device manufacturer, drug manufacturer, device trade name, keyword]

22. npv.mp. [mp=title, abstract, subject headings, heading word, drug trade name, original title, device manufacturer, drug manufacturer, device trade name, keyword]

23. diagnosis.mp. [mp=title, abstract, subject headings, heading word, drug trade name, original title, device manufacturer, drug manufacturer, device trade name, keyword]

24 . 15 or 16 or 17 or 18 or 19 or 20 or 21 or 22 or 23

25. axilla*.mp. [mp=title, abstract, subject headings, heading word, drug trade name, original title, device manufacturer, drug manufacturer, device trade name, keyword]

26. axilla.mp. [mp=title, abstract, subject headings, heading word, drug trade name, original title, device manufacturer, drug manufacturer, device trade name, keyword]

27. lymph.mp. [mp=title, abstract, subject headings, heading word, drug trade name, original title, device manufacturer, drug manufacturer, device trade name, keyword]

28. lymph*.mp. [mp=title, abstract, subject headings, heading word, drug trade name, original title, device manufacturer, drug manufacturer, device trade name, keyword] 
29. 25 or 26 or 27 or 28

3o. node.mp. [mp=title, abstract, subject headings, heading word, drug trade name, original title, device manufacturer, drug manufacturer, device trade name, keyword]

31. metastasis.mp. [mp=title, abstract, subject headings, heading word, drug trade name, original title, device manufacturer, drug manufacturer, device trade name, keyword]

32. nodes.mp. [mp=title, abstract, subject headings, heading word, drug trade name, original title, device manufacturer, drug manufacturer, device trade name, keyword]

33. 30 or 31 or 32

34. neoadjuvant.mp. [mp=title, abstract, subject headings, heading word, drug trade name, original title, device manufacturer, drug manufacturer, device trade name, keyword]

35. primary.mp. [mp=title, abstract, subject headings, heading word, drug trade name, original title, device manufacturer, drug manufacturer, device trade name, keyword]

36. preoperative.mp. [mp=title, abstract, subject headings, heading word, drug trade name, original title, device manufacturer, drug manufacturer, device trade name, keyword]

37. therapy.mp. [mp=title, abstract, subject headings, heading word, drug trade name, original title, device manufacturer, drug manufacturer, device trade name, keyword]

38. immunotherapy.mp. [mp=title, abstract, subject headings, heading word, drug trade name, original title, device manufacturer, drug manufacturer, device trade name, keyword]

39. chemotherapy.mp. [mp=title, abstract, subject headings, heading word, drug trade name, original title, device manufacturer, drug manufacturer, device trade name, keyword]

40. systemic.mp. [mp=title, abstract, subject headings, heading word, drug trade name, original title, device manufacturer, drug manufacturer, device trade name, keyword]

41. 34 or 35 or 36

42.37 or 38 or 39 or 40

43. 41 and 42

44. 10 and 14 and 24 and 29 and 33 and 43

\section{Pubmed search strategy}

(((()((("Breast Neoplasms"[Mesh]) OR ((((((carcinoma) OR neoplasm) OR neoplasms) OR malignancy) OR adenocarcinoma) OR cancer $))$ ) AND (((breast OR mamma $))$ OR ((((((carcinoma) OR neoplasm) OR neoplasms) OR 
malignancy) OR adenocarcinoma) OR cancer))) AND ((("Sentinel Lymph Node Biopsy"[Mesh]) OR sentinel lymph node biopsy) OR slnb)) AND ((((((()ensitivity) OR specificity)) OR ((("Sensitivity and Specificity"[Mesh])) OR "Predictive Value of Tests"[Mesh])) OR negative predictive value) OR positive predictive value) OR likelihood ratio) OR diagnosis)) AND ((((("Lymph Nodes"[Mesh]) OR "Lymphatic Vessels"[Mesh]) OR "Lymphatic Metastasis"[Mesh]) OR "Lymphatic System"[Mesh]) OR "Axilla"[Mesh]) OR axilla)) AND (((("Neoadjuvant Therapy"[Mesh]) OR neoadjuvant) OR preoperative) OR primary)) AND ((("Antineoplastic Agents"[Mesh]) OR chemotherapy) OR immunotherapy) OR systemic therapy) 


\section{Appendix 2.2}

\section{Results of bivariate meta-regression analyses}

\section{Identification rate}

\begin{tabular}{lccc}
\hline Covariates & Number of studies & Coefficient & $P$-value \\
\hline Year of publication & 7 & -0.004 & 0.895 \\
Study design & 7 & -0.027 & 0.369 \\
Sample size & 7 & 0.029 & 0.304 \\
Clinical nodal stage & 6 & 0.015 & 0.582 \\
\hline
\end{tabular}

Meta-regression analyses for the outcome identification rate were obtained using covariates, which included year of publication (2013-2015 versus 2007-2012), study design (retrospective versus prospective study design), sample size of study population (less versus more than 100 patients), clinical nodal stage (clinical nodal stage $1-2$ versus clinical nodal stage $1-3$ ).

\section{False-negative rate}

\begin{tabular}{lccc}
\hline Covariates & Number of studies & Coefficient & P-value \\
\hline Year of publication & 8 & -0.042 & 0.306 \\
Study design & 8 & -0.067 & 0.079 \\
Sample size & 8 & -0.037 & 0.383 \\
Clinical nodal stage & 7 & 0.086 & 0.049 \\
Definition isolated tumour cells & 7 & -0.019 & 0.678 \\
Additional immunohistochemistry & 8 & -0.034 & 0.458 \\
\hline
\end{tabular}

Meta-regression analyses for the outcome false-negative rate were obtained using covariates, which included year of publication (2013-2015 versus 2007-2012), study design (retrospective versus prospective study design), sample size of study population (less versus more than 100 patients), clinical nodal stage (clinical nodal stage 1-2 versus clinical nodal stage 1-3), definition of isolated tumour cells (isolated tumour cells considered as true negative versus isolated tumour cells considered as false negative), additional immunohistochemistry lymph nodes (no versus yes). 


\section{Appendix 2.3}

a. Forest plot of axillary pathological complete response

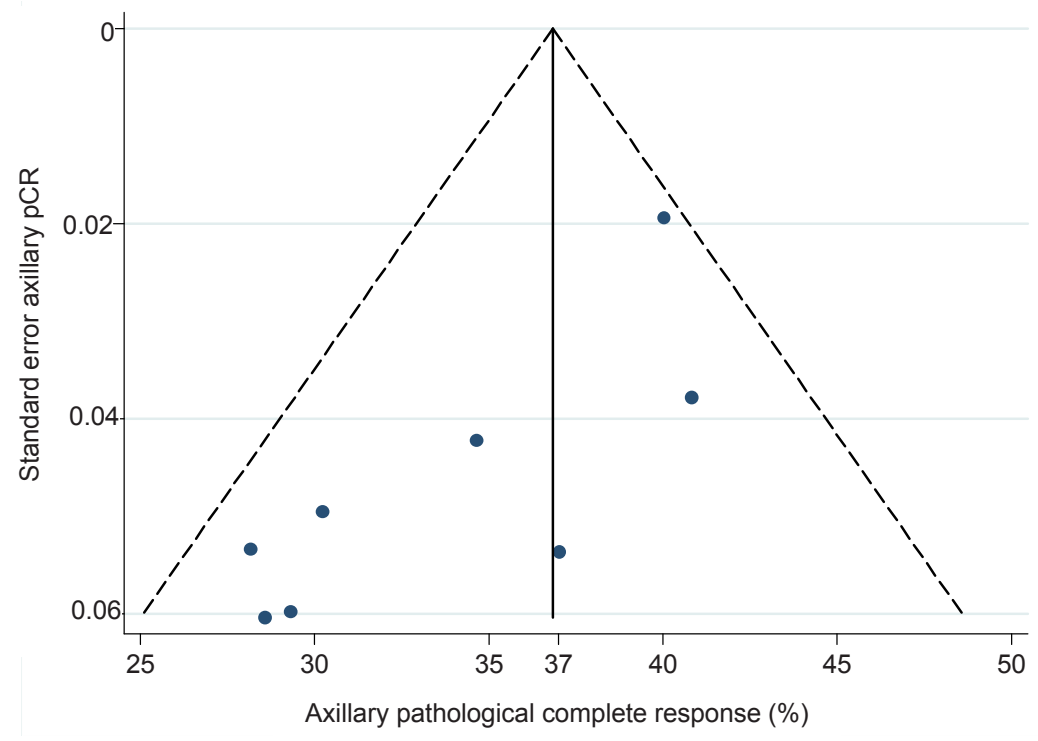

3b. Funnel plot of axillary pathological complete response

\begin{tabular}{|c|c|c|c|}
\hline Study (year) & Effect $\mathrm{Si}_{2}$ & $(95 \% \mathrm{Cl})$ & Weight (\%) \\
\hline Boileau (2015) & & $0.35(0.26,0.43)$ & 10.06 \\
\hline Boughey (2013) & & $0.40(0.36,0.44)$ & 47.60 \\
\hline Brown (2010) & & $0.30(0.21,0.40)$ & 7.31 \\
\hline Kang (2011) & & $0.29(0.18,0.41)$ & 5.02 \\
\hline Ozmen (2010) & & $0.28(0.18,0.39)$ & 6.29 \\
\hline Park (2013) & & $0.41(0.33,0.48)$ & 12.55 \\
\hline Shen (2007) & & $0.29(0.17,0.40)$ & 4.92 \\
\hline Yagata (2013) & & $0.37(0.27,0.48)$ & 6.23 \\
\hline
\end{tabular}

Forest plot and funnel plot with pseudo 95\% confidence intervals of axillary pathological complete response. The pooled axillary pCR was $36.8 \%\left(I^{2}=41.5 \%\right.$, $P=0.102)$. 


\section{Chapter}

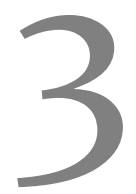

A novel less invasive approach for axillary staging after neoadjuvant chemotherapy in patients with axillary node positive breast cancer by combining Radioactive Iodine Seed localisation in the Axilla with the Sentinel node procedure (RISAS): a Dutch prospective multicentre validation study

TJA van Nijnatten*, JM Simons*, ML Smidt, CC van der Pol, PJ van Diest, A Jager, D van Klaveren, BLR Kam, MBI Lobbes, M de Boer, C Verhoef, LB Koppert", EJT Luiten ${ }^{\#}$ *\# These authors contributed equally to this work Clin Breast Cancer. 2017;17(5):399-402 


\section{Abstract}

\section{Background}

In one out of three patients with initial node positive $(\mathrm{cN}+)$ breast cancer neoadjuvant chemotherapy (NAC) results in an axillary pathologic complete response (ax-pCR). This urges the need for a less invasive axillary staging method. Recently introduced less invasive procedures are insufficient in accurately identifying ax-pCR. Therefore, we propose a novel less invasive axillary staging procedure, i.e. the RISAS procedure: Radioactive Iodine Seed localisation in the Axilla with the Sentinel node procedure, a combination of the MARI procedure and sentinel lymph node biopsy (SLNB).

\section{Patients and methods}

In this open single arm multicentre validation study $225 \mathrm{cN}+$ (biopsy proven) patients will undergo the RISAS procedure, in which a positive lymph node is marked by an I-125 seed prior to NAC. After completion of NAC, this I-125 seed marked lymph node is removed together with (additional) sentinel lymph node(s). The RISAS procedure is subsequently followed by a completion ALND. RISAS nodes will be compared to lymph nodes of the completion ALND specimen. Primary endpoint is accuracy of the RISAS procedure. Identification rate, false negative rate, negative predictive value and possible concordance between the MARI and sentinel lymph node(s) will be reported.

\section{Conclusion}

This prospective multicentre RISAS trial will enable us to validate the combination of MARI and SLNB for assessing axillary response to NAC in $\mathrm{cN}_{+}$ patients. If RISAS proves to be an accurate axillary staging procedure, ALND may safely be abandoned in case of ax-pCR confirmed by the RISAS procedure. 


\section{Introduction}

Surgical management of the axilla in breast cancer evolved greatly in the past decades. In clinically node negative patients axillary staging shifted from axillary lymph node dissection (ALND) to sentinel lymph node biopsy (SLNB). In clinically node positive patients $(\mathrm{cN}+)$ ALND is still recommended for axillary staging. ${ }^{1,2}$ However, the shift from adjuvant to neoadjuvant chemotherapy (NAC) causes a growing need for a less invasive axillary staging approach, since NAC enables downstaging of axillary disease. ${ }^{3,4}$ In up to $36 \%$ of all patients, conversion of $\mathrm{cN}+$ to axillary pathologic complete response (ax-pCR) as a result of NAC is reported. ${ }^{5}$ In case of HER2neu positive patients, ax-pCR is even achieved in up to $74 \%$ of patients when NAC is combined with HER2-targeted therapy. ${ }^{6,7}$ Patients in whom ax-pCR is achieved are not expected to benefit from ALND, but a significant proportion will suffer from side effects. Consequently, routine ALND is increasingly being regarded as overtreatment in these patients.

Until now, imaging techniques have not been able to accurately identify ax$\mathrm{pCR}$. As for surgical techniques, various less invasive axillary staging procedures have been proposed for $\mathrm{cN}+$ patients after completion of NAC. The SLNB seems to be insufficient with reported false negative rates (FNRs) ranging from $7 \%$ to $25 \%{ }^{8,9}$ Since SLNB aims to identify patients with ax-pCR without missing residual disease, a negative predictive value (NPV) of $95 \%$ or higher is desirable. A high NPV is especially important since residual tumour is considered resistant to the administered chemotherapy and may require additional treatment. However, NPVs for SLNB do not exceed $86 \%,{ }^{5}$ which means that residual axillary disease is missed in 1 out of every 6 patients in whom ax-pCR is suggested by the sentinel lymph node(s) (SLN(s)). Factors contributing to the improvement of FNR/NPV of SLNB are use of the dual tracer technique and removal of at least 3 SLNs. ${ }^{8,10-12}$ In daily practice, the latter is not always achievable, as was demonstrated by the SENTINA trial in which most patients had only 1 to 2 SLN(s) removed. ${ }^{12}$ Donker et al. introduced an alternative, less invasive axillary staging procedure: the MARI procedure (Marking Axillary lymph nodes with Radioactive Iodine seeds). ${ }^{13}$ In this procedure, an I-125 seed is placed in the biopsy proven positive axillary lymph node prior to NAC. After completion of NAC, the I-125 seed-containing node is removed at time of breast surgery. In 98 pre-treatment $\mathrm{cN}+$ patients an identification rate of $97 \%$ and a FNR of $7 \%$ was reported. MARI has a reported NPV of $83 \%$, thus residual axillary disease is still overlooked to the same extent as with SLNB. ${ }^{13,14}$ In conclusion, both MARI and SLNB remain insufficiently accurate as solitary staging procedures.

Caudle et al. proposed Targeted Axillary Dissection (TAD), ${ }^{15}$ involving clip placement in a biopsy proven positive axillary lymph node prior to NAC followed by localisation of this clipped node with an I-125 seed after completion of NAC. 
Excision of the I-125 seed marked node was combined with excision of SLN(s). All $\mathrm{cN}+$ patients treated with NAC and in whom the biopsied node was marked with a clip were included in a prospective registry. Retrospectively, eighty-five patients that underwent TAD followed by a completion ALND were identified. A FNR of $2 \%$ and a NPV of $97 \%$ were reported. ${ }^{15}$ These results are promising; however, the study is hampered by a single centre study design and a small sample size with only 35 patients in whom ax-PCR was achieved. Consequently, widespread implementation of TAD is not recommended before a large, preferably multicentre, validation study is performed.

Therefore, we propose a multicentre, open, single arm validation study in which the RISAS procedure, which consists of Radioactive Iodine Seed localisation in the Axilla combined with the SLNB, is investigated. In order to validate RISAS, a completion ALND will be performed in all patients. The I-125 seed is placed prior to the start of NAC. If this study proves that RISAS accurately predicts axillary nodal status after completion of NAC, the ALND and its concomitant morbidity may safely be omitted and prevented in patients with ax-pCR predicted by RISAS.

\section{Patients and methods}

The RISAS study is an open single arm non-inferiority multicentre validation study to assess accuracy of RISAS for axillary staging after NAC in pre-treatment $\mathrm{cN}+$ breast cancer patients. Nodal positivity is always confirmed by pathological assessment before start of NAC in case of suspicious nodes on axillary ultrasound. Patients will be recruited in approximately eight participating hospitals in The Netherlands over a period of two years. Each hospital has at least one year of experience with I-125 seed-guided localisation. Given a non-inferiority margin of $6.25 \%$ and assuming a prevalence of a positive ALND of $64 \%$, a total of 225 patients is needed in order to test non-inferiority of RISAS compared with ALND. Patients fulfilling all eligibility criteria will - after completion of NAC undergo the RISAS procedure and subsequent completion ALND (Table 3.1). Patients in whom I-125 seed placement is not possible and in whom the RISAS procedure is not followed by completion ALND will be excluded from final analysis. Chemotherapy regimens will be determined according to current Dutch breast cancer guidelines ${ }^{1}$ and multidisciplinary team decision making. In HER2neu positive patients NAC is combined with HER2-targeted therapy.

The RISAS procedure consists of the MARI-procedure combined with SLNB. First, the most suspicious node is sampled by means of core needle biopsy or fine needle aspiration in order to confirm nodal positivity. Subsequently, the MARIprocedure is performed. This consists of marking the pathologically proven 
positive lymph node with an I-125 seed prior to the start of NAC under ultrasound guidance. Retrieval of the sampled node is facilitated by reporting multiple characteristics of this node, e.g. diameter of the node and diameter of the cortex. Furthermore, the radiologist will capture an image of the biopsied lymph node. Iodine seeds with a maximum activity of $7.4 \mathrm{MBq}(0.2 \mathrm{mCi})$ are used. ${ }^{16}$ SLNB will be performed according to the standard dual tracer technique. After excision of the RISAS node(s), the surgeon will proceed with the completion ALND. The RISAS node(s) and the remaining nodes of the axillary basin will be secured separately.

Table $3.1 \quad$ Eligibility criteria.

\begin{tabular}{ll}
\hline Inclusion criteria & Exclusion criteria \\
\hline Female, age $\geq 18$ years & Pregnancy or nursing \\
Invasive breast cancer with biopsy proven & Contraindications to RISAS (e.g. allergic reaction \\
positive axillary lymph node(s) & to blue dye) \\
NAC & Recurrent breast cancer \\
& History of axillary surgery or radiotherapy \\
& Positive infra- and / or supraclavicular lymph nodes \\
& Distant metastasis \\
\hline
\end{tabular}

Abbreviations: NAC neoadjuvant chemotherapy, RISAS Radioactive Iodine Seed localisation in the Axilla combined with the Sentinel node procedure.

The pathologist will assess the RISAS nodes and remaining nodes of the completion ALND specimen separately. Presence of isolated tumour cells, micrometastases and / or macrometastases will be registered. Routine H\&E staining will be performed on all nodes. Immunohistochemistry will only be performed on each RISAS node, which is negative on H\&E staining, irrespective of ALND specimen findings.

This study has been approved by the Institutional Review Board of the Erasmus Medical Centre (MEC-2016-412) and registered at ClinicalTrials.gov (NCTo28oo317).

Basic statistical analysis will be used. The test outcome of both RISAS and completion ALND can be either negative or positive. If the test outcome of RISAS is positive, the test outcome of the completion ALND will also be considered positive. The outcomes will be formulated in a $2 \times 2$ contingency table. The primary endpoint of our study is accuracy of the RISAS procedure and will be defined by calculating sensitivity, NPV and FNR. 


\section{Discussion}

With the growing need for less invasive axillary staging after NAC, various procedures have been proposed. MARI and SLNB remain insufficient for this purpose since they miss potentially therapy resistant disease to a rather high extent. ${ }^{5,11,13}$ TAD shows promising results, but due to the retrospective analysis of patients and small sample size of this single centre study it seems premature to implement this procedure as standard practice and replace ALND. ${ }^{15}$ Our present RISAS study will provide answers to questions regarding $\mathrm{TAD}$, the role of immunohistochemistry and what to do if fewer than two nodes will be removed. ${ }^{17}$ An overview of the studies proposing different less invasive axillary staging procedures is provided in Table 3.2.

Table 3.2 Overview of studies comparing less invasive surgical procedures with axillary lymph node dissection for axillary staging after NAC in pre-treatment $\mathrm{cN}+$ patients.

\begin{tabular}{|c|c|c|c|c|}
\hline & ACOSOG Z1071 ${ }^{11}$ & MARI-procedure $^{13}$ & TAD $^{15}$ & RISAS \\
\hline Type of procedure & SLNB & MARI & $\begin{array}{l}\text { SLNB + I-125 seed } \\
\text { placement in the } \\
\text { clipped node after } \\
\text { completion of NAC }\end{array}$ & $\begin{array}{c}\text { SLNB + I-125 seed } \\
\text { placement prior to } \\
\text { NAC }\end{array}$ \\
\hline Sample size ${ }^{*}$ & 687 & 98 & 85 & 225 \\
\hline Prevalence axillary ax-pCR & 40.0 & 26.0 & 41.0 & $\#$ \\
\hline Identification rate (\%) & 92.7 & 97.0 & 100 & $\#$ \\
\hline FNR (\%) & 14.7 & 7.0 & 2.0 & $\#$ \\
\hline NPV (\%) & 82.0 & 83.0 & 97.2 & \# \\
\hline
\end{tabular}

* This includes all patients in whom concomitant ALND is performed, " pending on results.

Abbreviations: SLNB sentinel lymph node biopsy, MARI Marking the Axillary lymph node with Radioactive Iodine seeds, TAD Targeted Axillary Dissection, RISAS Radioactive Iodine seed localisation in the Axilla combined with Sentinel node procedure, I-125 seed iodine-125 seed, NAC neoadjuvant chemotherapy, ax-pCR pathologic complete response, FNR false-negative rate, $N P V$ negative predictive value.

Since RISAS combines MARI and SLNB, the accuracy of RISAS is supposed not to be hindered by shortcomings of the solitary techniques. Metastatic lymph nodes may block and deviate normal lymphatic drainage, resulting in "false SLN(s)". Given the fact that the RISAS procedure not only retrieves (false) SLNs but also pre-treatment pathologically proven metastatic lymph nodes it has the potential to be equal to ALND in staging of the axilla. Caudle et al. already demonstrated that the clipped node was not retrieved as an SLN in $23 \%$ of patients. ${ }^{15}$

An advantage of RISAS compared to TAD is that the biopsy proven positive node is primarily marked by an I-125 seed and not preceded by clip placement. Exact placement of the I-125 seed directly adjacent to the clip may be challenging, thus possibly hampering accuracy. Furthermore, direct marking of the lymph node by I-125 seed obviates an extra hospital visit after completion of NAC, which 
minimizes patient burden, and it obviates an additional procedure, which reduces logistic issues.

\section{Conclusion}

The RISAS study is a multicentre prospective non-inferiority study to validate the combination of MARI and SN for predicting axillary response to NAC in pretreatment $\mathrm{cN}+$ patients. The ultimate goal is to identify ax-pCR patients by a minimally invasive procedure, in order to prevent unnecessary ALND with its associated side effects without comprising oncologic safety. ALND may then be rendered as management procedure only for patients with residual axillary disease. 


\section{References}

1. Richtlijn Mammacarcinoom, NABON 2012.

2. National Comprehensive Cancer Network. NCCN clinical practice guidelines in oncology: Breast Cancer. V.1.2015.

3. Boughey JC, McCall LM, Ballman KV, et al. Tumor biology correlates with rates of breastconserving surgery and pathologic complete response after neoadjuvant chemotherapy for breast cancer: findings from the ACOSOG Z1o71 (Alliance) Prospective Multicenter Clinical Trial. Ann Surg 2014;260:608-14; discussion 14-6.

4. Cortazar P, Zhang L, Untch M, et al. Pathological complete response and long-term clinical benefit in breast cancer: the CTNeoBC pooled analysis. Lancet 2014;384:164-72.

5. van Nijnatten TJ, Schipper RJ, Lobbes MB, Nelemans PJ, Beets-Tan RG, Smidt ML. The diagnostic performance of sentinel lymph node biopsy in pathologically confirmed node positive breast cancer patients after neoadjuvant systemic therapy: A systematic review and metaanalysis. Eur J Surg Oncol 2015;41:1278-87.

6. Dominici LS, Negron Gonzalez VM, Buzdar AU, et al. Cytologically proven axillary lymph node metastases are eradicated in patients receiving preoperative chemotherapy with concurrent trastuzumab for HER2-positive breast cancer. Cancer 2010;116:2884-9.

7. van la Parra RF, Kuerer HM. Selective elimination of breast cancer surgery in exceptional responders: historical perspective and current trials. Breast Cancer Res 2016;18:28.

8. Mamounas EP, Brown A, Anderson S, et al. Sentinel node biopsy after neoadjuvant chemotherapy in breast cancer: results from National Surgical Adjuvant Breast and Bowel Project Protocol B-27. J Clin Oncol 2005;23:2694-702.

9. Shen J, Gilcrease MZ, Babiera GV, et al. Feasibility and accuracy of sentinel lymph node biopsy after preoperative chemotherapy in breast cancer patients with documented axillary metastases. Cancer 2007;109(7):1255-63.

10. Boileau JF, Poirier B, Basik M, et al. Sentinel node biopsy after neoadjuvant chemotherapy in biopsy-proven node-positive breast cancer: the SN FNAC study. J Clin Oncol 2015;33:258-64.

11. Boughey JC, Suman VJ, Mittendorf EA, et al. Sentinel lymph node surgery after neoadjuvant chemotherapy in patients with node-positive breast cancer: the ACOSOG Z1o71 (Alliance) clinical trial. JAMA 2013;310:1455-61.

12. Kuehn T, Bauerfeind I, Fehm T, et al. Sentinel-lymph-node biopsy in patients with breast cancer before and after neoadjuvant chemotherapy (SENTINA): a prospective, multicentre cohort study. Lancet Oncol 2013;14:609-18.

13. Donker M, Straver ME, Wesseling J, et al. Marking axillary lymph nodes with radioactive iodine seeds for axillary staging after neoadjuvant systemic treatment in breast cancer patients: the MARI procedure. Ann Surg 2015;261:378-82.

14. Vugts G, Nieuwenhuijzen GA, Maaskant-Braat AJ, Schipper RJ, Smidt ML. Axillary Response Monitoring After Neoadjuvant Chemotherapy in Breast Cancer: Can We Avoid the Morbidity of Axillary Treatment? Ann Surg 2016;263:e28-9.

15. Caudle AS, Yang WT, Krishnamurthy S, et al. Improved Axillary Evaluation Following Neoadjuvant Therapy for Patients With Node-Positive Breast Cancer Using Selective Evaluation of Clipped Nodes: Implementation of Targeted Axillary Dissection. J Clin Oncol 2016;34:1072-8.

16. Straver ME, Loo CE, Alderliesten T, Rutgers EJ, Vrancken Peeters MT. Marking the axilla with radioactive iodine seeds (MARI procedure) may reduce the need for axillary dissection after neoadjuvant chemotherapy for breast cancer. Br J Surg 2010;97:1226-31.

17. Fedor DM, Landercasper J. Can a seed-sized tool from Texas spare clinically node positive breast cancer patients from a complete axillary dissection? Gland Surg 2016;5:450-2. 


\section{Part II}

Non-invasive lymph node staging 



\section{Chapter

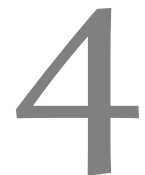

Routine use of standard breast MRI compared

to axillary ultrasound for differentiating

between no, limited and advanced axillary

nodal disease in newly diagnosed breast

cancer patients

TJA van Nijnatten, EH Ploumen, RJ Schipper, B Goorts, EH Andriessen, S Vanwetswinkel, M Schavemaker, P Nelemans, B de Vries, RGH Beets-Tan, ML Smidt, MBI Lobbes

Eur J Radiol 2016;85:2288-2294 


\section{Abstract}

\section{Objectives}

To compare standard breast MRI to dedicated axillary ultrasound (with or without tissue sampling) for differentiating between no, limited and advanced axillary nodal disease in breast cancer patients.

\section{Methods}

All patients who underwent breast MRI and dedicated axillary ultrasound between 2009 and 2014 were eligible. Exclusion criteria were recurrent disease, neoadjuvant systemic therapy and not receiving completion axillary lymph node dissection after positive sentinel lymph node biopsy (SLNB). Two radiologists independently reassessed all MRI exams. Axillary ultrasound findings were retrospectively collected. Probability of advanced axillary nodal disease ( $\mathrm{pN}_{2}-3$ ) given clinically node negative $(\mathrm{cNo})$ or limited $(\mathrm{cN} 1)$ findings was calculated, with corresponding negative predictive value (NPV) to exclude $\mathrm{pN}_{2}-3$ and positive predictive value (PPV) to identify axillary nodal disease. Histopathology served as gold standard.

\section{Results}

A total of 377 cases resulted in $81.4 \%$ no, 14.4\% limited and 4.2\% advanced axillary nodal disease at final histopathology. Probability of $\mathrm{pN}_{2}-3$ given cNo for breast MRI and axillary ultrasound was $0.7-0.9 \%$ versus $1.5 \%$ and probability of $\mathrm{pN2}-3$ given $\mathrm{cN}_{1}$ was $11.6-15.4 \%$ versus $29.0 \%$. When $\mathrm{cN}_{1}$ on breast MRI was observed, PPV to identify positive axillary nodal disease was $50.7 \%$ and $59.0 \%$.

\section{Conclusions}

Evaluation of axillary nodal status on standard breast MRI is comparable to dedicated axillary ultrasound in breast cancer patients. In patients who underwent preoperative standard breast MRI, axillary ultrasound is only required in case of suspicious nodal findings on MRI. 


\section{Introduction}

Nowadays, preoperative axillary nodal staging in breast cancer patients consists of axillary ultrasound with concomitant tissue sampling if indicated. ${ }^{1}$ Although, detection of every positive node was important in the past, ${ }^{2}$ recent studies such as the ACOSOG Zoo11, IBCSG 23-01 and AATRM 048/13/200o trials have shown that completion axillary lymph node dissection (ALND) after the detection of limited axillary nodal disease (i.e. 1-3 positive nodes) does not improve prognosis. ${ }^{3-5}$ As a consequence, excluding advanced axillary nodal disease (i.e. more than 3 positive nodes) rather than detecting clinically node positive disease is becoming increasingly important.

Previous studies addressing the diagnostic performance of axillary ultrasound reported a negative predictive value (NPV) of $96 \%$ to exclude advanced nodal disease in case of node negative findings. ${ }^{6,7}$ However, axillary ultrasound appears unable to differentiate between limited and advanced axillary nodal disease in case of positive axillary ultrasound findings, with a reported NPV of $50 \%{ }^{8}$

According to a recent systematic review, MRI might be a promising noninvasive nodal staging tool for preoperative evaluation of the axilla to determine node negative and node positive disease in breast cancer patients. ${ }^{9}$ However, the publications studied in this review did not investigate the ability of MRI to determine the number of positive nodes for differentiating between no, limited and advanced axillary nodal disease. Only a few studies compared MRI to ultrasonography, the most frequently used imaging modality for this purpose in daily practice. ${ }^{10}$ If preoperative MRI, which is often part of the diagnostic work-up of newly diagnosed breast cancer patients, could improve nodal staging, axillary treatment could be even more patient-tailored. Thus, the aim of this study was to investigate the diagnostic performance of standard breast MRI compared to dedicated axillary ultrasound to differentiate between no, limited and advanced axillary nodal disease, with pathology serving as the gold standard.

\section{Materials and methods}

\section{Patient selection}

All patients diagnosed between 2009 and 2014 with invasive breast cancer who underwent both standard breast MRI and axillary ultrasound prior to surgery were eligible for this study. Exclusion criteria were recurrent breast cancer, patients primarily treated with neoadjuvant systemic therapy or patients with a positive sentinel lymph node biopsy (SLNB) who did not receive a completion ALND. Due to the retrospective design of this study, the necessity to acquire informed 
consent from the study subjects was waived by the local medical ethics committee.

\section{Standard breast MRI exam and image analysis}

All included patients underwent a standard breast MRI protocol to evaluate breast cancer extent and the presence of contralateral breast cancer. Breast MRI was performed in prone position on two different 1.5 T MRI scanners (Ingenia and Intera, Philips Healthcare, Best, the Netherlands). Both MRI scanners used a body coil, which was in 2011 replaced by a dedicated 16-channel breast coil. Evaluation of the axillary lymph nodes was performed on a non-enhanced $3 \mathrm{D}$ T2W Turbo Spin Echo sequence. This sequence is part of the standard breast MRI protocol, which further consisted of dynamic, contrast-enhanced TiW sequence protocols using fat saturation and diffusion weighted imaging (DWI). Over the years, the MR sequence protocols were only slightly changed (Appendix 4.1).

A resident in radiology (M.S.) with two years of experience in breast radiology, pre-screened all breast MRI exams on technical quality criteria. Eligibility criteria were inclusion of the full axillary region including for example the sternoclavicular joint, axillary vein and the latissimus dorsi muscle. In addition, the axillary region had to be free of motion artefacts or inadequate signal-to-noise-ratio.

All axillary lymph nodes (i.e. axillary level 1-3, without assessment of the periclavicular and/or internal mammary lymph nodes) within one patient were separately counted and qualitatively assessed by two expert breast radiologists with 7 years (M.B.I.L.) and 5 years (S.V.) of breast imaging experience, respectively. Both radiologists were blinded for each other's results and to the histopathological outcome of the tumour and lymph nodes. However, radiologists were aware of the clinical tumour size, as assessed on mammography, ultrasound and/or breast MRI, similar to clinical practice.

Each individual lymph node was scored according to the criteria previously defined by Baltzer et al., using a confidence scale from o (no lymph nodes) to 4 (definitely malignant). "Suspicious characteristics included irregular margins, inhomogeneous cortex, perifocal edema, absent fatty hilum, asymmetry, and absence of chemical shift artefacts (Figure 4.1). ${ }^{11-13}$

Clinical axillary nodal staging was based on the number of suspicious axillary lymph nodes: none (cNo), limited ( $\mathrm{cN}_{1}, 1-3$ suspicious lymph nodes) and advanced ( $\mathrm{cN}_{2}-3,>3$ suspicious lymph nodes) axillary nodal disease. In patients diagnosed with bilateral invasive breast cancer, lymph nodes were assessed in both axillae separately. 


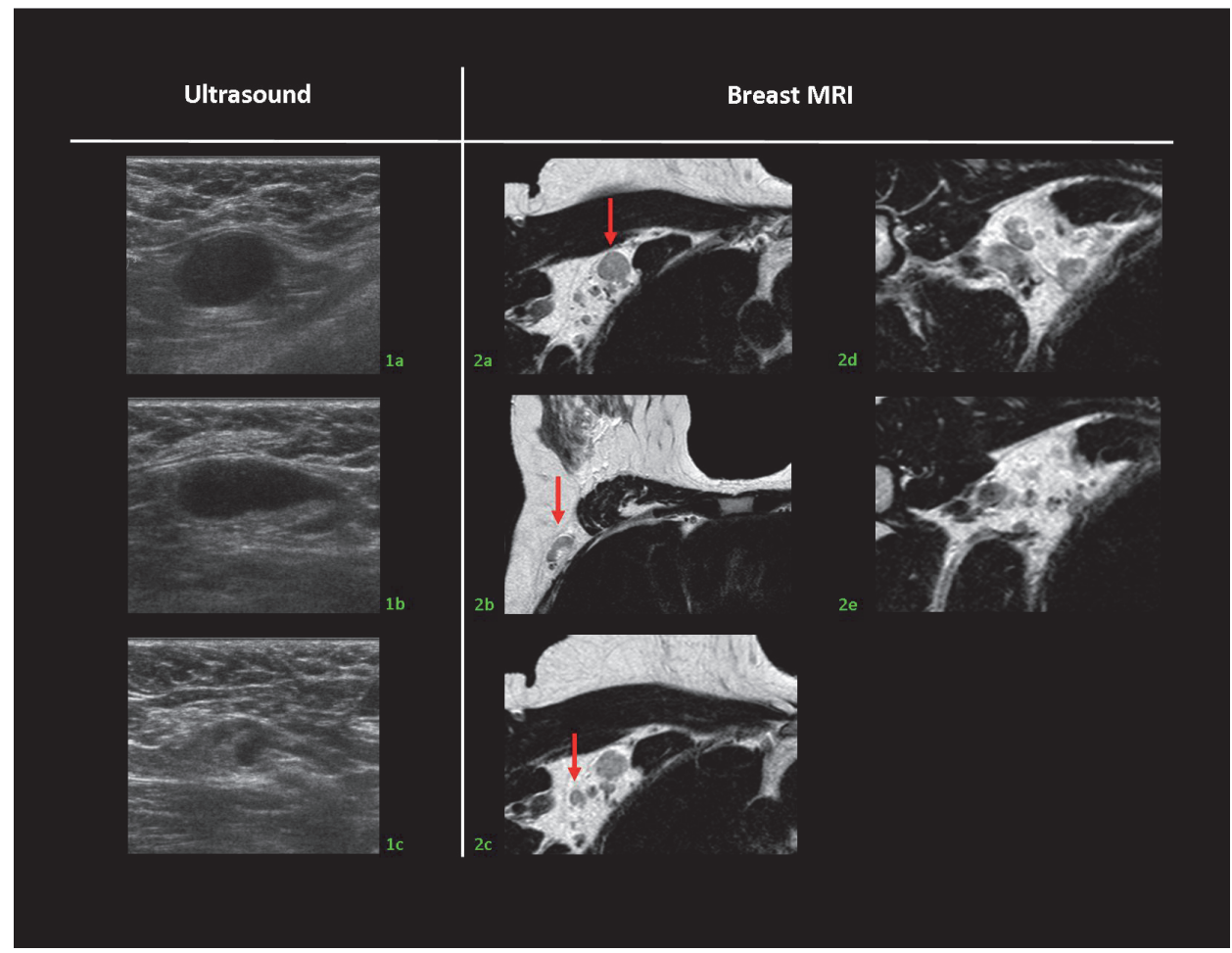

Figure 4.1 Transversal T2-weighted images of right axillary region of a female patient with a $45 \mathrm{~mm}$ large invasive ductal carcinoma in her right breast. Dedicated axillary ultrasound reported three suspicious axillary nodes $\left(\mathrm{cN}_{1}\right)$, whereas on MRI the radiologists predicted respectively 10 and 7 suspicious axillary lymph nodes (both $\mathrm{cN}_{2}-3$ ). The red arrows on MR Images $2 \mathrm{a}-\mathrm{c}$ represent the corresponding suspicious nodes detected during axillary ultrasound on a-c. The MR Images 2d and 2e demonstrate additional suspicious axillary lymph nodes. Histopathology of the axillary lymph node dissection reported a total of 9 axillary lymph node metastases (pN2-3).

\section{Axillary ultrasound}

Axillary ultrasound exams were performed by dedicated breast radiologists, using an ATL-HDI50oo system in combination with a linear 5 to $12-\mathrm{MHz}$ array transducer, which in 2011 was replaced by an iU22-xMATRIX ultrasound system with a linear 2 to $17-\mathrm{MHz}$ array transducer (both systems: Philips Healthcare, Best, the Netherlands). Criteria for a suspicious axillary lymph node on axillary ultrasound consisted of diffuse cortical thickening, focal cortical mass and/or thickening and loss of the fatty hilum. ${ }^{6}$ The result of these exams, including the number of suspicious axillary lymph nodes, was extracted from the radiology report for each individual patient. Tissue sampling was performed in case of suspicious axillary lymph nodes using 16-18 gauge core needle biopsy. When core 
needle biopsy was challenging, fine needle aspiration cytology was used. In cases of multiple suspicious nodes, only the most suspicious node was sampled and the number of suspicious nodes was reported. ${ }^{8,14}$ In this study, dedicated axillary ultrasound was defined as axillary ultrasound with or without tissue sampling if deemed necessary by the radiologist on call.

In contrast to MRI, clinically node positive disease with ultrasound was always histopathologically confirmed. Diagnostic performance of axillary ultrasound was described earlier by Schipper et al., by using the same study subjects between 2009 and $2012(\mathrm{n}=243) .^{8}$ In our current study, axillary ultrasound results were used for per-patient comparison with results of breast MRI.

\section{Surgical nodal staging}

In clinically node negative patients, based on negative axillary ultrasound findings or sampled tissue without evidence of tumour cells, SLNB was performed. The sentinel lymph node was identified by using a triple technique consisting of lymphoscintigraphy (using 8o MBq Technetium-99 m nanocolloid injected periareolar), blue dye to detect lymphatic vessels (Bleu Patente V; Guerbet, Aulnaysous-Bois, France), and a gamma probe to detect radioactivity. In case of one or more histopathologically confirmed metastases, a completion level I-II ALND was performed. In clinically node positive patients, based on tissue sampling with evidence of tumour cells after suspicious axillary ultrasound findings, ALND was performed.

\section{Histopathological evaluation}

Sentinel lymph nodes were sliced with a maximum thickness of $3 \mathrm{~mm}$ and paraffin embedded for histological evaluation. Step-sectioning at 500 micrometer intervals was done at three levels and were stained with haematoxylin and eosin (H\&E). If conventional H\&E slices were negative, a mouse anti-human MNF116 antibody (Dako, Glostrup, Denmark) was used for cytokeratin immunohistochemical staining.

All lymph nodes harvested by axillary lymph node dissection were paraffin embedded after $24 \mathrm{~h}$ of fixation for histological evaluation. Lymph nodes larger than $5 \mathrm{~mm}$ were sliced at a maximum thickness of $3 \mathrm{~mm}$, and stained with H\&E. Each node was recorded as either benign or as isolated tumour cells $(\leq 0.2 \mathrm{~mm})$, micrometastasis $(0.2 \leq 2.0 \mathrm{~mm})$ or macrometastasis $(>2.0 \mathrm{~mm}){ }^{.5}$ In this study, isolated tumour cells and micrometastases were considered negative. Pathological axillary nodal staging was based on the number of malignant axillary lymph nodes: none ( $\mathrm{pNo}$ ), limited ( $\mathrm{pN} 1,1-3$ lymph node metastases) and advanced ( $\mathrm{pN2}$ 3, >3 lymph node metastases) axillary nodal disease. 


\section{Statistical analysis}

Statistical analyses were performed by using Statistical Package for the Social Sciences software (version 22, IBM, Armonk, New York, USA). The probability of presence of advanced axillary nodal disease $\left(\mathrm{pN}_{2}-3\right)$ in case of test results cNo or $\mathrm{CN}_{1}$, was calculated as $\mathrm{pN}_{2}-3 /\left(\mathrm{pNo}^{2} \mathrm{pN}_{1}+\mathrm{pN}_{2}-3\right)$ for respectively cNo and $\mathrm{cN}_{1}$. In case of test result $\mathrm{cN}_{2}-3$, the probability of correctly predicted $\mathrm{pN}_{2}-3$ was calculated as $\mathrm{pN}_{2}-3 / \mathrm{cN}_{2}-3$. Discrepant cases between MRI and axillary ultrasound were described, to investigate whether results of both techniques could further decrease the probability of $\mathrm{pN}_{2}-3$ given $\mathrm{cNo}$ or $\mathrm{CN}_{1}$. A subgroup analysis between patients who underwent standard breast MRI between 2009 and 2010 (using a body coil) and 2011-2014 (using a dedicated breast coil) was performed to investigate whether there were any differences between both subgroups.

Interobserver agreement between the two readers reviewing the breast MRI exams, according to the initial confidence scale $0-4$, was calculated by quadratic weighted kappa coefficient $(\kappa)$ to determine the agreement between both readers for the evaluation of axillary nodal staging on standard breast MRI. ${ }^{16}$ A score of o2 was considered benign, a score of 3-4 malignant.

\section{Results}

Between 2009 and 2014, 803 patients were diagnosed with invasive breast cancer and underwent standard breast MRI and axillary ultrasound. After screening all MRI exams, a total of 377 MRI exams (6o.6\% of all eligible MRI exams) in 373 patients were included, due to bilateral breast cancer in four patients (Figure 4.2). Patient and tumour characteristics are shown in detail in Table 4.1.

Final pathologic report showed pNo in 81.4\%, $\mathrm{pN}_{1}$ in $14.4 \%$ and $\mathrm{pN}_{2}-3$ in $4.2 \%$ of the patients (Table 4.2). Clinical nodal status on MRI and ultrasound with corresponding pathological nodal status is shown in Table 4.2. Interobserver agreement between the radiologists on standard breast MRI was considered substantial ( $\kappa=0.68,95 \%$ CI $0.58-0.77)$.

\section{Negative axillary findings (cNo)}

With ultrasound, 344 cases were staged as cNo, of which 307 were pNo (89.2\%), 32 were $\mathrm{pN}_{1}(10.0 \%)$ and 5 were $\mathrm{pN}_{2}-3(1.5 \%)$. Thus, probability of $\mathrm{pN}_{2}-3$ given cNo was $1.5 \%$, with a corresponding NPV of $98.5 \%$ (95\% CI 96.4-99.5\%) to exclude advanced axillary nodal disease in case of cNo (Table 4.3).

According to reader 1, a total of 321 breast MRI exams were staged as cNo, of which 286 were $\mathrm{pNo}(89.1 \%), 32$ were $\mathrm{pN} 1(10.0 \%)$ and 3 were $\mathrm{pN}_{2-3}(0.9 \%)$. Thus, probability of $\mathrm{pN}_{2-3}$ given cNo was $0.9 \%$, with a corresponding NPV of $99.1 \%$ 
(95\% CI 97.1-99.8\%) to exclude advanced axillary nodal disease in case of cNo. In case of cNo according to reader 1 on MRI with $\mathrm{pN}_{2}-3$ as histopathological result $(\mathrm{n}=3)$, axillary ultrasound predicted 2 cases as $\mathrm{CN}_{1}$ and one as $\mathrm{cNo}$.

Reader 2 staged a total of 299 breast MRI exams as cNo, of which 273 were pNo (91.3\%), 24 were $\mathrm{pN}_{1}(8.0 \%)$ and 2 were $\mathrm{pN}_{2}-3$ (0.7\%). Probability of pN2-3 given cNo was $0.7 \%$, with a corresponding NPV of $99.3 \%$ (95\% CI 97.3-99.9\%) to exclude advanced axillary nodal disease in case of cNo. In case of cNo according to reader 2 on MRI with $\mathrm{pN}_{2}-3$ as histopathological result $(\mathrm{n}=2)$, axillary ultrasound predicted one case as $\mathrm{CN}_{1}$ and one cNo.

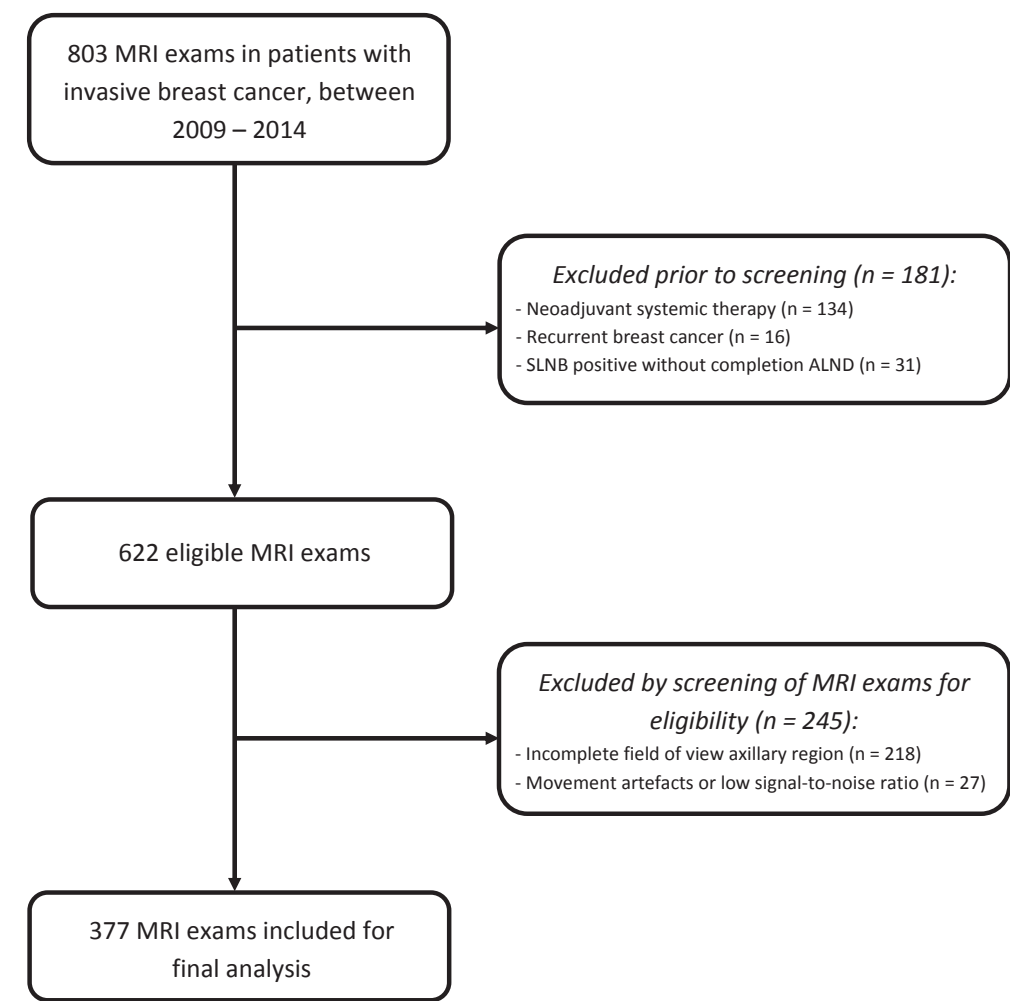

Figure 4.2 Flowchart of patient inclusion. Abbreviations: SLNB sentinel lymph node biopsy, ALND axillary lymph node dissection. 
Table 4.1 General patient and tumour characteristics.

\begin{tabular}{|c|c|}
\hline Number of patients & 377 \\
\hline Mean age (y) (range) & $58(21-81)$ \\
\hline Mean clinical tumour size (mm) (range) & $25.0(2-100)$ \\
\hline \multicolumn{2}{|l|}{ cT stage $(\%)$} \\
\hline $\mathrm{T}_{1}$ & $209(55.4)$ \\
\hline $\mathrm{T}_{2}$ & $133(35 \cdot 3)$ \\
\hline $\mathrm{T}_{3}$ & $35(9 \cdot 3)$ \\
\hline \multicolumn{2}{|l|}{ Uni- and multifocal tumours (\%) } \\
\hline Unifocal & $314(83.3)$ \\
\hline Multifocal & $63(16.7)$ \\
\hline \multicolumn{2}{|l|}{ Tumour type (\%) } \\
\hline Ductal & $311(82.5)$ \\
\hline Lobular & $42(11.1)$ \\
\hline Mixed ductal and lobular & $10(2.7)$ \\
\hline Other $^{\#}$ & $14(3.7)$ \\
\hline \multicolumn{2}{|l|}{ Tumour grade (\%) } \\
\hline 1 & $97(25 \cdot 7)$ \\
\hline 2 & $173(45.9)$ \\
\hline 3 & $104(27.6)$ \\
\hline Missing & $3(0.8)$ \\
\hline \multicolumn{2}{|l|}{ pT stage (\%) } \\
\hline Tis & $5(1.3)$ \\
\hline $\mathrm{T}_{1}$ & $268(71.1)$ \\
\hline $\mathrm{T}_{2}$ & $89(23.6)$ \\
\hline $\mathrm{T}_{3}$ & $13(3.5)$ \\
\hline $\mathrm{T}_{4}$ & $2(0.5)$ \\
\hline \multicolumn{2}{|l|}{ Hormonal and receptor status (\%) } \\
\hline ER/PR positive, Her2 negative & $284(75 \cdot 3)$ \\
\hline ER/PR negative, Her2 positive & $16(4.2)$ \\
\hline Triple negative & $39(10.4)$ \\
\hline Triple positive & $35(9 \cdot 3)$ \\
\hline Not determined & $3(0.8)$ \\
\hline \multicolumn{2}{|l|}{ Axillary surgery (\%) } \\
\hline SLNB & $292(77.5)$ \\
\hline SLNB with completion ALND & $49(13.0)$ \\
\hline ALND & $36(9.5)$ \\
\hline
\end{tabular}

Abbreviations: $c T$ stage clinical tumour stage, $p T$ stage pathological tumour stage, $p N$ stage pathological nodal stage, $S L N B$ sentinel lymph node biopsy, $A L N D$ axillary lymph node dissection. \# Other tumour types: ductal carcinoma in situ, mucinous carcinoma, adenoid cystic carcinoma and papillary carcinoma.

\section{Limited axillary findings $(\mathrm{cN})$}

According to ultrasound, 31 cases were staged as $\mathrm{cN}_{1}$, of which 22 were $\mathrm{pN}_{1}(71.0 \%)$ and 9 were $\mathrm{pN}_{2-3}$ (29.0\%). Thus, underestimation of $\mathrm{pN}_{2}-3$ given $\mathrm{cN}_{1}$ was $29.0 \%$, with a corresponding NPV of $71.0 \%(95 \%$ CI $51.8-85.1 \%)$ to exclude advanced axillary nodal disease in case of $\mathrm{cN}_{1}$ (Table 4.3).

According to reader 1, a total of 39 breast MRI exams were staged as $\mathrm{CN}_{1}$, of which 16 were pNo (41.0\%), 17 were $\mathrm{pN}_{1}(43.6 \%)$ and 6 were $\mathrm{pN}_{2-3}(15.4 \%)$. Thus, 
PPV to identify positive axillary nodal disease was 59.0\% (95\% CI 42.2-74.0\%) and probability of $\mathrm{pN}_{2}-3$ given $\mathrm{cN}_{1}$ was $15.4 \%$, resulting in a corresponding NPV of $84.6 \%$ (95\% CI 68.8-93.6\%) to exclude advanced axillary nodal disease in case of cN1. In case of $\mathrm{CN}_{1}$ according to reader 1 on MRI with $\mathrm{pN}_{2}-3$ as histopathological result $(n=6)$, axillary ultrasound predicted three cases as $\mathrm{cN}_{1}$ and three cases as cNo.

Reader 2 staged 69 breast MRI exams as $\mathrm{cN}_{1}$, of which 34 were pNo (49.3\%), 27 were $\mathrm{pN}_{1}(39.1 \%)$ and 8 were $\mathrm{pN}_{2}-3$ (11.6\%). PPV to identify positive axillary nodal disease was $50.7 \%(95 \%$ CI $38.5-62.9 \%)$ and probability of $\mathrm{pN}_{2}-3$ given $\mathrm{cN}_{1}$ was $11.6 \%$, resulting in a NPV of $88.4 \%$ (95\% CI $77.9-94.5 \%)$ to exclude advanced axillary nodal disease in case of $\mathrm{cN}_{1}$. In case of $\mathrm{cN}_{1}$ according to reader 2 on MRI with $\mathrm{pN}_{2-3}$ as histopathological result $(\mathrm{n}=8)$, axillary ultrasound predicted five cases as $\mathrm{cN}_{1}$ and three cases as cNo.

Table 4.2 Clinical nodal stage according to ultrasound- and MRI findings, with corresponding pathological nodal stage.

\begin{tabular}{|c|c|c|c|c|c|c|}
\hline Axillary pN stage & $\begin{array}{c}\mathrm{pNo} \\
(\mathrm{n}=\mathbf{2 4 1})\end{array}$ & $\begin{array}{l}\mathrm{pNoi+} \\
(\mathrm{n}=35)\end{array}$ & $\begin{array}{c}\mathrm{pN}_{1}(\mathrm{mi}) \\
(\mathrm{n}=31)\end{array}$ & $\begin{array}{c}\mathrm{pN}_{1} \\
(\mathrm{n}=54)\end{array}$ & $\begin{array}{l}\mathrm{pN}_{2-3} \\
(\mathrm{n}=16)\end{array}$ & $\begin{array}{c}\text { Total } \\
(\mathrm{n}=377)\end{array}$ \\
\hline \multicolumn{7}{|l|}{ MRI reader 1} \\
\hline cNo & 226 & 34 & 26 & 32 & 3 & 321 \\
\hline $\mathrm{cN}_{1}$ & 11 & 1 & 4 & 17 & 6 & 39 \\
\hline $\mathrm{cN}_{2}-3$ & 4 & - & 1 & 5 & 7 & 17 \\
\hline \multicolumn{7}{|l|}{ MRI reader 2} \\
\hline cNo & 216 & 30 & 27 & 24 & 2 & 299 \\
\hline $\mathrm{cN}_{1}$ & 25 & 5 & 4 & 27 & 8 & 69 \\
\hline $\mathrm{CN}_{2}-3$ & - & - & - & 3 & 6 & 9 \\
\hline \multicolumn{7}{|l|}{ Ultrasound } \\
\hline cNo & 241 & 35 & 31 & 32 & 5 & 344 \\
\hline $\mathrm{cN}_{1}$ & - & - & - & 22 & 9 & 31 \\
\hline $\mathrm{CN}_{2}-3$ & - & - & - & - & 2 & 2 \\
\hline
\end{tabular}

Abbreviations: $c N$ clinical nodal stage, $p N$ pathological nodal stage, $p N o i+$ isolated tumour cells, $p N_{1}(m i)$ micrometastases

Table 4.3 Probability of $\mathrm{pN}_{2}-3$ given $\mathrm{cNo}, \mathrm{cN}_{1}$ or $\mathrm{cN}_{2}-3$, with corresponding NPV to exclude advanced axillary nodal disease and PPV to identify axillary nodal disease.

\begin{tabular}{lccc}
\hline & MRI reader 1 & MRI reader 2 & Ultrasound \\
\hline$c N o$ & $\mathrm{n}=321$ & $\mathrm{n}=299$ & $\mathrm{n}=344$ \\
Underestimation $\mathrm{pN2}-3$ & $0.7 \%(2 / 299)$ & $1.5 \%(5 / 344)$ \\
$\mathrm{NPV}$ & $0.9 \%(3 / 321)$ & $99.3 \%(297 / 299)$ & $98.5 \%(339 / 344)$ \\
$c N_{1}$ & $99.1 \%(318 / 321)$ & $\mathrm{n}=69$ & $\mathrm{n}=31$ \\
Underestimation $\mathrm{pN2}-3$ & $\mathrm{n}=39$ & $11.6 \%(8 / 69)$ & $29.0 \%(9 / 31)$ \\
$\mathrm{NPV}$ & $15.4 \%(6 / 39)$ & $88.4 \%(61 / 69)$ & $71.0 \%(22 / 31)$ \\
PPV & $84.6 \%(33 / 39)$ & $50.7 \%(35 / 69)$ & $100 \%(31 / 31)$ \\
$c N_{2}-3$ & $59.0 \%(23 / 39)$ & $\mathrm{n}=9$ & $\mathrm{n}=2$ \\
Correctly identified $\mathrm{pN2}-3$ & $\mathrm{n}=17$ & $66.7 \%(6 / 9)$ & $100 \%(2 / 2)$ \\
\hline
\end{tabular}

Abbreviations: $c N$ clinical nodal stage, $N P V$ negative predictive value, $P P V$ positive predictive value. 


\section{Advanced axillary findings ( $\left.\mathrm{CN}_{2}-3\right)$}

According to breast MRI, reader 1 and reader 2 correctly predicted 7 (41.2\%) and $6(66.7 \%)$ cases with $\mathrm{pN2}-3$ out of 17 and 9 patients as $\mathrm{CN}_{2}-3$ according to MRI, whereas ultrasound predicted $2(100 \%)$ cases with $\mathrm{pN}_{2}-3$ out of 2 patients as $\mathrm{cN}_{2}-3$ according to ultrasound (Table 4.3).

\section{Comparison MR protocols 2009-2010 and 2011-2014}

In November 2010, the MR protocols for standard breast MRI changed (Appendix 4.1). In this study, 127 MRI exams were performed between 2009 and 2010 and 250 between 2011 and 2014. In case of cNo, this resulted in the following probability of pN2-3 given cNo: $0.9 \%$ versus $0.9 \%$ for reader 1 and $1.0 \%$ versus $0.5 \%$ for reader 2 . In case of $\mathrm{cN}_{1}$, this resulted in the following probability of $\mathrm{pN}_{2}-3$ given $\mathrm{cN}_{1}: 14.3 \%$ versus $16.0 \%$ for reader 1 and $13.6 \%$ versus $10.6 \%$ for reader 2 .

\section{Discussion}

As axillary treatment based on clinical nodal status is becoming important, accurate assessment of the number of axillary nodal metastases is mandatory. Recent studies demonstrated axillary ultrasound is insufficiently accurate for differentiating between $\mathrm{CN}_{1}$ and $\mathrm{cN}_{2}-3$, while other studies suggest that MRI could be of additional value for proper axillary nodal staging. ${ }^{9,17}$ Studies concerning axillary nodal staging with MRI were performed differentiating between axillary node negative and positive disease, instead of no, limited and advanced axillary nodal disease. We showed that axillary nodal staging on standard breast MRI is comparable to axillary ultrasound.

The importance of differentiating between limited and advanced axillary nodal disease, is based on its therapeutic consequences: locoregional radiation therapy. It has been known that the addition of locoregional radiation therapy to chemotherapy improves the prognosis of patients with advanced axillary nodal disease. ${ }^{18,19}$ However, the omission of additional treatment (completion ALND) in cNo patients with limited axillary nodal disease at final pathology, does not seem to affect prognosis..$^{3-5,14}$ As a consequence, differentiation between limited and advanced axillary nodal disease is becoming clinically more relevant in order to identify patients who benefit from additional axillary treatment, since both surgical and radiation therapy cause side effects as well. ${ }^{20}$

According to the current guideline of the European Society of Medical Oncology for primary breast cancer, axillary ultrasound is recommended for the assessment of regional lymph nodes. ${ }^{18}$ Previous studies concerning axillary ultrasound in breast cancer patients reported an inability to differentiate between 
limited and advanced axillary nodal disease, because in $50-68 \%$ of the $\mathrm{cN}_{1}$ patients detected with ultrasound pathologically confirmed advanced axillary nodal disease was still present. ${ }^{8,17}$ Yet, a recent study by Verheuvel et al. demonstrated a worse prognosis in node positive patients detected by ultrasound compared to SLNB-positive patients, indicating that preoperative axillary ultrasound remains important. ${ }^{21}$ In short, axillary ultrasound cannot differentiate between no, limited and advanced axillary nodal disease accurately, but it might be of benefit to differentiate between prognostic favourable and infavourable patients.

Since many breast cancer patients undergo preoperative breast MRI to assess the extent of the disease in the breast, we studied whether lymph node staging would be more accurate using this imaging exam compared to axillary ultrasound. The added benefit of using standard breast MRI would be that no extra exams, such as dedicated axillary MR imaging, would be necessary. In this study, the nonenhanced T2 weighted sequence was chosen for axillary nodal staging, due to better anatomical characterization of the axillary lymph nodes compared to dynamic contrast-enhanced sequence. This is supported by a systematic review of Kuijs et al. who demonstrated that non-enhanced $\mathrm{T}_{2} \mathrm{~W}$ sequence was one of the most accurate sequence protocols for axillary lymph node imaging in breast cancer patients, however the included studies did only differentiate between node negative and positive findings instead of differentiating between limited and advanced axillary nodal disease in addition. ${ }^{9}$

Recently, Hyun et al. performed a retrospective study to evaluate the use of breast MRI to differentiate between no, limited and advanced axillary disease. They showed similar percentages of probability of $\mathrm{pN}_{2}-3$ given cNo and $\mathrm{cN}_{1}$ in a cohort of 310 patients treated with primary surgery: $0.4 \%$ for cNo and $15.6 \%$ for $\mathrm{CN}_{1}$, compared to $0.7-0.9 \%$ and $11.6-15.6 \%$ in our study. ${ }^{22}$ In our opinion, this study had an important limitation, since it did not directly compare breast MRI results with the results of axillary ultrasound in a per-patient analysis, thereby missing important information to compare the results of the proposed new test (breast MRI) with the standard reference test (axillary ultrasound). Our results demonstrated a decrease in probability of $\mathrm{pN}_{2}-3$ given $\mathrm{CN}_{1}$ from $29.0 \%$ to $11.6-$ $15.4 \%$, when axillary ultrasound was replaced by breast MRI. Yet, breast MRI resulted in a relatively low PPV of $50.7 \%$ and $59.0 \%$ to identify node positive disease in case $\mathrm{cN}_{1}$ was observed. Consequently, the benefit of MRI in $\mathrm{cN}_{1}$ patients is limited. Besides, in cases of $\mathrm{cN}_{1}$ predicted by standard breast MRI with $\mathrm{pN}_{2}-3$ as histopathological result, axillary ultrasound did not further decrease the probability of $\mathrm{pN}_{2}-3$, yet second-look axillary ultrasound after suspicious axillary lymph nodes on MRI can be recommended due to improved selection of cNo. ${ }^{23}$ Second-look axillary ultrasound is performed different compared to the axillary ultrasound in our study, because of the information of suspicious nodes on MRI. 
In cN2-3 patients, MRI correctly predicted more patients with $\mathrm{pN}_{2-3}$ compared to ultrasound, but because of the small sample size these results should be interpreted with caution.

This study had several limitations. First, predictive values (PPV and NPV) depend on prevalence of axillary nodal disease. ${ }^{24}$ Therefore, results should be interpreted with the prevalence of $\mathrm{pN}_{1}$ and $\mathrm{pN}_{2}-3$ in mind, which consisted of $18.6 \%$ of the patients with macrometastases at final histopathology in our cohort.

Second, there is a small chance of verification bias, due to referral of patients to biopsy based on test results of axillary ultrasound. Axillary treatment in our study population depends on clinical nodal status according to the axillary ultrasound: in case of node negative findings patients will receive SLNB, in case of node positive findings patients will receive ALND. Previous studies reported false negative rates of SLNB up to $9.8 \%$, indicating that patients with a negative SLNB still had positive lymph nodes in the completion ALND. ${ }^{25}$ As a consequence, this might have affected our results, as some patients with a negative SLNB could have positive lymph nodes in the remaining axillary lymph nodes.

Third, due to differences in prevalence of $\mathrm{cNo}, \mathrm{cN}_{1}$ and $\mathrm{cN}_{2}-3$ between MRI (respectively for reader 1 and 2) and ultrasound, statistical analyses to compare these results together were not possible. As a consequence, MRI results of reader 1 and 2 could not be combined, as some cases were differently assessed by both readers. However, this study showed that MRI resulted in many false positive findings in case of $\mathrm{cN} 1$, indicating that MRI is not able to replace axillary ultrasound for preoperative nodal staging. In contrast to MRI, axillary ultrasound has no false positive results because of the histopathological results of the biopsy.

Fourth, many patients were excluded according to the applied exclusion criteria. These criteria included all patients treated with neoadjuvant systemic therapy, in which a higher amount of patients with advanced axillary nodal disease is likely to be expected. Furthermore, nearly $40 \%$ of all eligible MRI exams had to be excluded due to inadequate visualization of the axilla on standard breast MRI. The fact that these exams were used to assess breast cancer extent within the breast is the reason behind this high exclusion rate, as these scans were not primarily focussing on axillary imaging.

Finally, we used two different MRI scanners with different sequence protocols. In November 2010 the MR sequence protocol was slightly adjusted due to the introduction of a dedicated 16-channel breast coil (Appendix 4.1). During that time, slice thickness was reduced and in plane resolution slightly increased on both systems. Despite these modifications, we did not observe differences in probability of $\mathrm{pN}_{2}-3$ in case of cNo or $\mathrm{cN} 1$.

In conclusion, standard breast MRI in preoperative axillary lymph node staging is comparable to dedicated axillary ultrasound. In patients who underwent 
preoperative standard breast MRI, axillary ultrasound is only required in case of suspicious nodal findings on MRI. 


\section{References}

1. Diepstraten SC, Sever AR, Buckens CF, Veldhuis WB, van Dalen T, van den Bosch MA, et al. Value of preoperative ultrasound-guided axillary lymph node biopsy for preventing completion axillary lymph node dissection in breast cancer: a systematic review and meta-analysis. Ann Surg Oncol 2014;21:51-9.

2. Giuliano AE, Jones RC, Brennan M, Statman R. Sentinel lymphadenectomy in breast cancer. J Clin Oncol 1997;15:2345-50.

3. Giuliano AE, Hunt KK, Ballman KV, Beitsch PD, Whitworth PW, Blumencranz PW, et al. Axillary dissection vs no axillary dissection in women with invasive breast cancer and sentinel node metastasis: a randomized clinical trial. JAMA 2011;305:569-75.

4. Galimberti V, Cole BF, Zurrida S, Viale G, Luini A, Veronesi P, et al. Axillary dissection versus no axillary dissection in patients with sentinel-node micrometastases (IBCSG 23-01): a phase 3 randomised controlled trial. Lancet Oncol 2013;14:297-305.

5. M. Sola, J.A. Alberro, M. Fraile, P. Santesteban, M. Ramos, R. Fabregas, et al., Complete axillary lymph node dissection versus clinical follow-up in breast cancer patients with sentinel node micrometastasis: final results from the multicenter clinical trial AATRM 048/13/200o. Ann Surg Oncol 2013;20:120-7.

6. C.H. Neal, C.P. Daly, A.V. Nees, M.A. Helvie, Can preoperative axillary US help exclude N2 and N3 metastatic breast cancer, Radiology 257 (2010) 335-41.

7. R.S. Jackson, C. Mylander, M. Rosman, R. Andrade, K. Sawyer, T. Sanders, et al., Normal axillary ultrasound excludes heavy nodal disease burden in patients with Breast cancer, Ann. Surg. Oncol. 22 (2015) 3289-95.

8. R.J. Schipper, L.M. van Roozendaal, B. de Vries, R.M. Pijnappel, R.G. Beets-Tan, M.B. Lobbes, et al., Axillary ultrasound for preoperative nodal staging in breast cancer patients: is it of added value, Breast 22 (2013) 1108-13.

9. V.J. Kuijs, M. Moossdorff, R.J. Schipper, R.G. Beets-Tan, E.M. Heuts, K.B. Keymeulen, et al., The role of MRI in axillary lymph node imaging in breast cancer patients: a systematic review, Insights Imaging 6 (2015) 203-15.

10. Y. Feng, R. Huang, Y. He, A. Lu, Z. Fan, T. Fan, et al., Efficacy of physical examination, ultrasound, and ultrasound combined with fine-needle aspiration for axilla staging of primary breast cancer, Breast Cancer Res. Treat.149 (2015) 761-5.

11. P.A. Baltzer, M. Dietzel, H.P. Burmeister, R. Zoubi, M. Gajda, O. Camara, et al., Application of MR mammography beyond local staging: is there a potential to accurately assess axillary lymph nodes? evaluation of an extended protocol in an initial prospective study, AJR Am. J. Roentgenol. 196 (2011) W641-7.

12. N. Farshchian, S. Tamari, N. Farshchian, H. Madani, M. Rezaie, H.R. Mohammadi-Motlagh, Diagnostic value of chemical shift artifact in distinguishing benign lymphadenopathy, Eur. J. Radiol. 80 (2011) 594-7.

13. D.M. Lambregts, L.A. Heijnen, M. Maas, I.J. Rutten, M.H. Martens, W.H. Backes,et al., Gadofosveset-enhanced MRI for the assessment of rectal cancer lymph nodes: predictive criteria, Abdom. Imaging 38 (2013) 720-7.

14. L.M. van Roozendaal, J.H. de Wilt, T. van Dalen, J.A. van der Hage, L.J. Strobbe, L.J. Boersma, et al., The value of completion axillary treatment in sentinel node positive breast cancer patients undergoing a mastectomy: a Dutch randomized controlled multicentre trial (BOOG 2013-07), BMC Cancer 15(2015) 610.

15. S.C. Lester, S. Bose, Y.Y. Chen, J.L. Connolly, M.E. de Baca, P.L. Fitzgibbons, et al., Protocol for the examination of specimens from patients with invasive carcinoma of the breast, Arch. Pathol. Lab. Med. 133 (2009) 1515-38. 
16. J. Cohen, Weighted kappa: nominal scale agreement with provision for scaled disagreement or partial credit, Psychol. Bull. 70 (1968) 213-20.

17. M. Pilewskie, S.K. Mautner, M. Stempel, A. Eaton, M. Morrow, Does a positive axillary lymph node needle biopsy result predict the need for an axillary lymph node dissection in clinically node-negative Breast cancer patients in the ACOSOG Zoon era? Ann. Surg. Oncol. 23 (April (4)) (2016) 1123-8.

18. E. Senkus, S. Kyriakides, S. Ohno, F. Penault-Llorca, P. Poortmans, E. Rutgers,et al., Primary breast cancer: ESMO Clinical Practice Guidelines for diagnosis, treatment and follow-up, Ann. Oncol. 26 (Suppl. 5) (2015) v8-30.

19. J. Ragaz, S.M. Jackson, N. Le, I.H. Plenderleith, J.J. Spinelli, V.E. Basco, et al., Adjuvant radiotherapy and chemotherapy in node-positive premenopausal women with breast cancer, $\mathrm{N}$. Engl. J. Med. 337 (1997) 956-62.

2o. R.L. Ahmed, A. Prizment, D. Lazovich, K.H. Schmitz, A.R. Folsom, Lymphedema and quality of life in breast cancer survivors: the Iowa Women's Health Study, J. Clin. Oncol. 26 (2008) 568996.

21. N.C. Verheuvel, I. van den Hoven, H.W. Ooms, A.C. Voogd, R.M. Roumen, The role of ultrasound-guided lymph node biopsy in axillary staging of invasive breast cancer in the postACOSOG Zoont trial era, Ann. Surg. Oncol. 22 (2015)409-15.

22. S.J. Hyun, E.K. Kim, H.J. Moon, J.H. Yoon, M.J. Kim, Preoperative axillary lymph node evaluation in breast cancer patients by breast magnetic resonance imaging (MRI): Can breast MRI exclude advanced nodal disease? Eur. Radiol.26 (November (11)) (2016) 3865-73.

23. S.J. Hyun, E.K. Kim, J.H. Yoon, H.J. Moon, M.J. Kim, Adding MRI to ultrasound and ultrasoundguided fine-needle aspiration reduces the false-negative rate of axillary lymph node metastasis diagnosis in breast cancer patients, Clin. Radiol. 70 (2015) 716-22.

24. D.A. Grimes, K.F. Schulz, Uses and abuses of screening tests, Lancet 359 (2002)881-4.

25. D.N. Krag, S.J. Anderson, T.B. Julian, A.M. Brown, S.P. Harlow, T. Ashikaga, et al., Technical outcomes of sentinel-lymph-node resection and conventional axillary-lymph-node dissection in patients with clinically node-negative breast cancer: results from the NSABP B-32 randomised phase III trial, Lancet Oncol. 8 (2007) 881-8. 


\section{Appendix 4.1}

\section{Overview MR protocols for standard breast MRI between 2009 -} 2014

MRI 1: Philips Intera 1.5T.

\begin{tabular}{lcccccc}
\hline \multirow{2}{*}{ Coil } & 2009 & 2010 & 2011 & 2012 & 2013 & 2014 \\
\cline { 2 - 6 } & $\begin{array}{c}\text { Sense body } \\
\text { coil }\end{array}$ & $\begin{array}{c}\text { Sense body } \\
\text { coil }\end{array}$ & $\begin{array}{c}\text { Sense breast } \\
\text { 16-channel }\end{array}$ & $\begin{array}{c}\text { Sense breast } \\
\text { 16-channel }\end{array}$ & $\begin{array}{c}\text { Sense breast } \\
\text { 16-channel }\end{array}$ & $\begin{array}{c}\text { Sense breast } \\
16 \text {-channel }\end{array}$ \\
\hline Matrix & $512 \times 512$ & $512 \times 512$ & $384 \times 384$ & $384 \times 384$ & $384 \times 384$ & $384 \times 384$ \\
$\begin{array}{l}\text { Pixel spacing } \\
\text { (mm) }\end{array}$ & $0.742^{2}$ & $0.645^{2}$ & $0.959^{2}$ & $0.959^{2}$ & $0.959^{2}$ & $0.959^{2}$ \\
$\begin{array}{l}\text { Field of View } \\
\text { (mm) }\end{array}$ & $380^{2}$ & $330^{2}$ & $368^{2}$ & $368^{2}$ & $368^{2}$ & $368^{2}$ \\
$\begin{array}{l}\text { In-plane resolution } \\
\text { (mm) }\end{array}$ & $0.7^{2}$ & $0.6^{2}$ & $1.0^{2}$ & $1.0^{2}$ & $1.0^{2}$ & $1.0^{2}$ \\
$\begin{array}{l}\text { Repetition time } \\
\text { (msec) }\end{array}$ & 5922 & 3012 & 2000 & 2000 & 2000 & 2000 \\
$\begin{array}{l}\text { Echo time } \\
\text { (msec) }\end{array}$ & 110 & 110 & 204 & 205 & 205 & 258 \\
$\begin{array}{l}\text { Flip angle } \\
\text { (degrees) }\end{array}$ & 90 & 90 & 90 & 90 & 90 & 90 \\
$\begin{array}{l}\text { Echo train length } \\
\text { Slice thickness }\end{array}$ & 33 & 33 & 80 & 80 & 80 & 96 \\
(mm) & 3.0 & 3.0 & 2.0 & 2.0 & 2.0 & 2.0 \\
\hline
\end{tabular}

MRI 2: Philips Ingenia 1.5T

\begin{tabular}{|c|c|c|c|c|c|c|}
\hline & 2009 & 2010 & 2011 & 2012 & 2013 & 2014 \\
\hline Coil & $\begin{array}{c}\text { Sense body } \\
\text { coil }\end{array}$ & $\begin{array}{c}\text { Sense body } \\
\text { coil }\end{array}$ & $\begin{array}{c}\text { Sense breast } \\
\text { 16-channel }\end{array}$ & $\begin{array}{c}\text { Sense breast } \\
\text { 16-channel }\end{array}$ & $\begin{array}{c}\text { Sense breast } \\
\text { 16-channel }\end{array}$ & $\begin{array}{c}\text { Sense breast } \\
\text { 16-channel }\end{array}$ \\
\hline Matrix & $512 \times 512$ & $512 \times 512$ & $400 \times 400$ & $400 \times 400$ & $400 \times 400$ & $400 \times 400$ \\
\hline $\begin{array}{l}\text { Pixel spacing } \\
(\mathrm{mm})\end{array}$ & $0.684^{2}$ & $0.606^{2}$ & $0.854^{2}$ & $0.854^{2}$ & $0.854^{2}$ & $0.854^{2}$ \\
\hline $\begin{array}{l}\text { Field of View } \\
(\mathrm{mm})\end{array}$ & $350^{2}$ & $310^{2}$ & $342^{2}$ & $342^{2}$ & $342^{2}$ & $342^{2}$ \\
\hline $\begin{array}{l}\text { In-plane resolution } \\
(\mathrm{mm})\end{array}$ & $0.7^{2}$ & $0.6^{2}$ & $0.9^{2}$ & $0.9^{2}$ & $0.9^{2}$ & $0.9^{2}$ \\
\hline $\begin{array}{l}\text { Repetition time } \\
\text { (msec) }\end{array}$ & 8213 & 6845 & 2000 & 2000 & 2000 & 2000 \\
\hline $\begin{array}{l}\text { Echo time } \\
\text { (msec) }\end{array}$ & 110 & 110 & 215 & 218 & 218 & 218 \\
\hline $\begin{array}{l}\text { Flip angle } \\
\left(^{\circ}\right)\end{array}$ & 90 & 90 & 90 & 90 & 90 & 90 \\
\hline Echo train length & 29 & 29 & 95 & 95 & 95 & 95 \\
\hline $\begin{array}{l}\text { Slice thickness } \\
(\mathrm{mm})\end{array}$ & 3.0 & 3.0 & 2.0 & 2.0 & 2.0 & 2.0 \\
\hline
\end{tabular}





\section{Chapter 5}

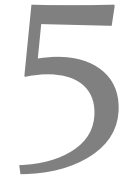

\section{The diagnostic performance of gadofosveset- enhanced axillary MRI for nodal (re)staging in breast cancer patients: results of a validation study}

T.J.A. van Nijnatten *, R-J. Schipper *, M.B.I. Lobbes, L.M. van Roozendaal, S. Vöö, M. Moossdorff, M-L. Paiman, B. de Vries, K.B.M.I. Keymeulen, J.E. Wildberger, M.L. Smidt \#, R.G.H. Beets-Tan \# *\# Contributed equally to this work Clin Radiol. 2017 [Epub ahead of print] 


\section{Abstract}

\section{Purpose}

To evaluate diagnostic performance of gadofosveset-enhanced MRI (GDF-MRI) in addition to T2-weighted MRI (T2W-MRI) for nodal (re)staging in newly diagnosed breast cancer patients.

\section{Methods}

Ninety patients underwent axillary T2W- and GDF-MRI. Two radiologists independently scored each lymph node; first on T2W-MRI, subsequently adjusting their score on GDF-MRI. Diagnostic performance parameters were calculated on node-by-node and patient-by-patient validation with histopathology as the reference standard. Furthermore, learning curve analysis for reading GDFMRI was performed.

\section{Results}

In patient-by-patient validation, overall reader 1 performance for $\mathrm{T} 2 \mathrm{~W}$ - and GDFMRI was similar with area under the receiver operating characteristic curves (AUC) of 0.75 and $0.77(P=0.731)$, while for reader 2 AUC-values were 0.79 and $0.72(P=0.156)$. For node-by-node validation, AUC-values of T2W- and GDF-MRI were 0.76 and $0.82(P=0.018)$ and 0.77 and $0.77(P=0.998)$ for reader 1 and 2 . The AUC for reader 1 of the first one-third nodes was 0.71 , improving to 0.80 and 0.95 for the next and last one-third. Sensitivity, specificity, PPV and NPV improved from $38 \%, 89 \%, 56 \%$, and $79 \%$ to $60 \%, 93 \%, 64 \%$ and $92 \%$. AUC of reader 2 improved from 0.69 to 0.79 .

\section{Conclusion}

The current study confirmed that GDF-MRI, in addition to T2W-MRI, has potential as non-invasive method for nodal (re)staging in breast cancer. 


\section{Introduction}

Breast cancer is one of the most frequently diagnosed cancers among women, with a worldwide incidence rate of 1.7 million. ${ }^{1}$ Survival rates have increased in the last decades due to improved diagnostic techniques and treatment regimens. Five year survival rates are up to $98 \%$ for early stage, lymph node negative breast cancer. ${ }^{2}$ Consequently, more attention is afforded to maintaining quality of life by limiting overtreatment and its associated lifetime morbidity.

For a long time, axillary lymph node dissection (ALND) was routinely performed to assess nodal status in breast cancer patients. This procedure is associated with significant short- and long-term morbidity. Seroma, lymphedema, nerve injury and reduced shoulder function are reported in up to $49 \%$ of the patients after 3 years of follow-up..$^{3-5}$ About 15 years ago, the sentinel lymph node biopsy (SLNB) became the standard procedure in clinically node negative patients. It was followed by a completion ALND in case of a positive sentinel lymph node(s). ${ }^{6}$ Nevertheless, SLNB remains an invasive procedure with shortterm side effects in $25 \%$ of patients and long-term morbidity (for example lymphedema) in up to $6 \%$ of the patients. ${ }^{4,7,8}$

As $74 \%$ of the sentinel lymph nodes show no metastasis, a non-invasive imaging technique able to identify these node negative patients would be a step forward in personalized treatment. ${ }^{9}$ It would result in a significant reduction of morbidity and increase the patient's quality of life, while maintaining the high survival rates achieved so far.

An accurate non-invasive nodal staging tool could also guide treatment in clinically node positive breast cancer patients. In cases of neoadjuvant systemic therapy, pathological complete response of axillary lymph node metastases is achieved in approximately $37 \% .^{10}$ Identifying these patients by a non-invasive imaging modality, and thereby avoiding an ALND, would further reduce breast cancer treatment related morbidity.

In rectal cancer, the use of magnetic resonance imaging (MRI) with a blood pool contrast agent, gadofosveset, improved diagnostic performance for lymph node staging. ${ }^{11,12}$ Consequently, a feasibility study of Schipper et al. on the use of gadofosveset-enhanced MRI for axillary nodal staging in ten breast cancer patients showed a sensitivity, specificity, positive predictive value (PPV) and negative predictive value (NPV) of $86 \%, 94 \%, 75 \%$ and $97 \%$, respectively. They concluded that gadofosveset-enhanced MRI is a promising tool to accurately detect node negative breast cancer patients. ${ }^{\text {II }}$

The aim of this study was to prospectively assess the diagnostic performance of gadofosveset-enhanced MRI for nodal (re)staging in a larger population of newly diagnosed breast cancer patients using histopathology as reference standard. 


\section{Materials and methods}

\section{Setting and patients}

The local medical ethics committee approved this prospective single centre study, which was performed from May 2012 until May 2016. Consecutive breast cancer patients were included after written informed consent was obtained. Inclusion criteria were patients with biopsy proven in situ or invasive breast cancer, scheduled for SLNB or ALND. Exclusion criteria were pregnancy, prior ipsilateral axillary surgery, a glomerular filtration rate $<45 \mathrm{~mL} / \mathrm{min} / 1.73 \mathrm{~m}^{2}$, and contraindications for either MRI or gadofosveset. GDF-MRI was performed shortly before SLNB or ALND in standard setting. In case of neoadjuvant systemic therapy, axillary MRI was performed between the last course of systemic therapy and SLNB and/or ALND. This study was registered at ClinicalTrials.gov (NCTo16o9920).

\section{Imaging}

Axillary imaging was performed on a 3.0 Tesla MRI scanner (Achieva 3.oTX, Philips Healthcare, Best, The Netherlands) using a 32-channel cardiac coil with the patient in supine position and ipsilateral arm elevated. The imaging protocol consisted of the following parameters: First, a three-dimensional $\left.{ }_{3} \mathrm{D}\right)$ standard $\mathrm{T}_{2}$ weighted $\left(\mathrm{T}_{2} \mathrm{~W}\right)$ turbo spin echo sequence without fat suppression was performed in 3 minutes 26 seconds (repetition time (TR) $2000 \mathrm{~ms}$; echo time (TE) $153 \mathrm{~ms} ; 90$ degree flip angle; turbo spin echo factor 50 ; number of signal acquisitions (NSA) 1; slice thickness $2.50 \mathrm{~mm}$; reconstruction section thickness $1.25 \mathrm{~mm}$; field of view (FOV) $220 \times 220 \mathrm{~mm}^{2}$; matrix $176 \times 176$; resulting in an in-plane resolution of $1.25 \times$ $\left.1.25 \mathrm{~mm}^{2}\right)$.

Second, gadofosveset (Ablavar ${ }^{\circ}$, Lantheus Medical Imaging, North Billerica, United States of America) contrast with a dose of $0.12 \mathrm{ml} / \mathrm{kg}$ bodyweight was intravenously administered, followed after 12 minutes by a $3 \mathrm{D}$ T1 weighted ( $\mathrm{T} 1 \mathrm{~W})$ gradient echo sequence in 5 minutes 7 seconds (TR $6.3 \mathrm{~ms}$, TE $4.6 \mathrm{~ms} ; 15$ degree flip angle; NSA 2; slice thickness $1.25 \mathrm{~mm}$; field-of-view $220 \mathrm{x} 220 \mathrm{~mm}^{2}$; matrix size $176 \times 176$; resulting in an in-plane resolution of $1.25 \times 1.25 \mathrm{~mm}^{2}$ ). The time interval of 12 minutes after contrast administration was chosen based on a previous publication."

\section{Image evaluation}

Two radiologists independently analyzed $\mathrm{T}_{2} \mathrm{~W}-\mathrm{MRI}$ and $\mathrm{T} 2 \mathrm{~W}$-MRI combined with gadofosveset-enhanced MRI (GDF-MRI), blinded for histopathological findings and each other's results. Similar to clinical practice, radiologists were aware of 
clinical tumour size, assessed on mammography, ultrasound and/or breast MRI. However, they were blinded for results of physical examination and axillary ultrasound. After each assessment on MRI, radiologists received direct feedback concerning final histopathology.

The first radiologist (R.B.T.) had 15 years of experience in pelvic MRI including eight years in GDF-MRI for lymph node assessment in rectal cancer, but no expertise in breast MRI [reader 1]. The second radiologist (M.L.) had seven years of experience in breast MRI and in axillary lymph node assessment using ultrasound and four years in axillary lymph node assessment on breast MRI, but no expertise in GDF-MRI [reader 2].

On T2W-MRI, each node was scored using a confidence level scale of o (definitely benign) to 4 (definitely malignant), using the criteria as described by Baltzer et al.. ${ }^{12}$ Suspicious characteristics were irregular margins, inhomogeneous cortex, perifocal edema and absent fatty hilum. Next, GDF-MRI was presented, and the radiologists were allowed to adjust their initial score. The GDF-MRI criteria were previously described by Lambregts et al. ${ }^{13}$; in short, a node was considered benign when it showed contrast hyperintensity after intravenous injection of gadofosveset. Malignant characteristics were absence of contrast hyperintensity and absence of an intact nodal border. Examples of imaging features of malignant lymph nodes on GDF-MRI are given in Figures 5.1 and 5.2.

A

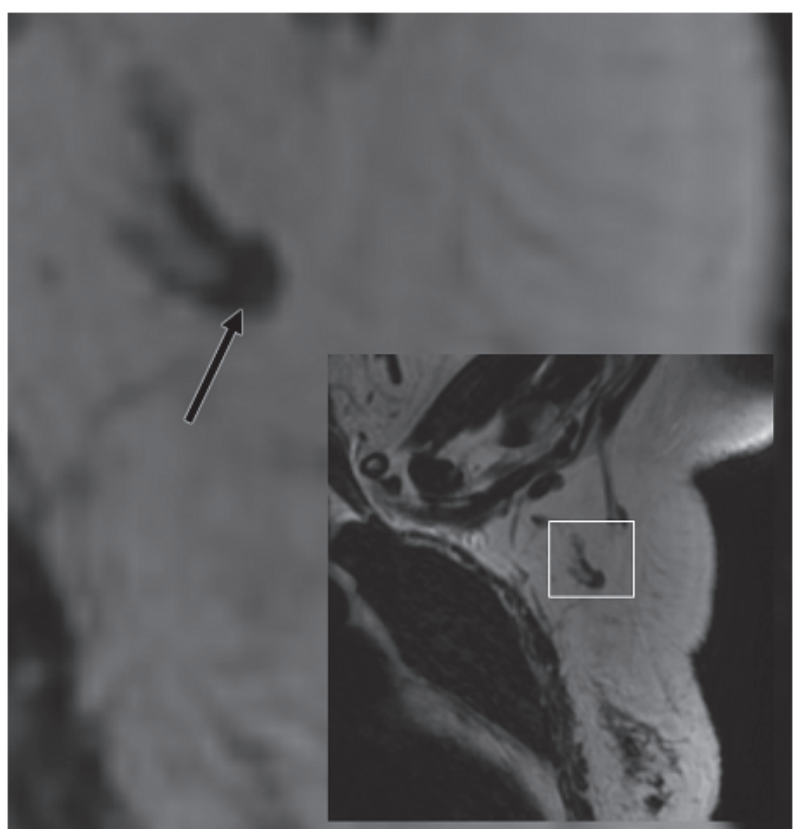


B

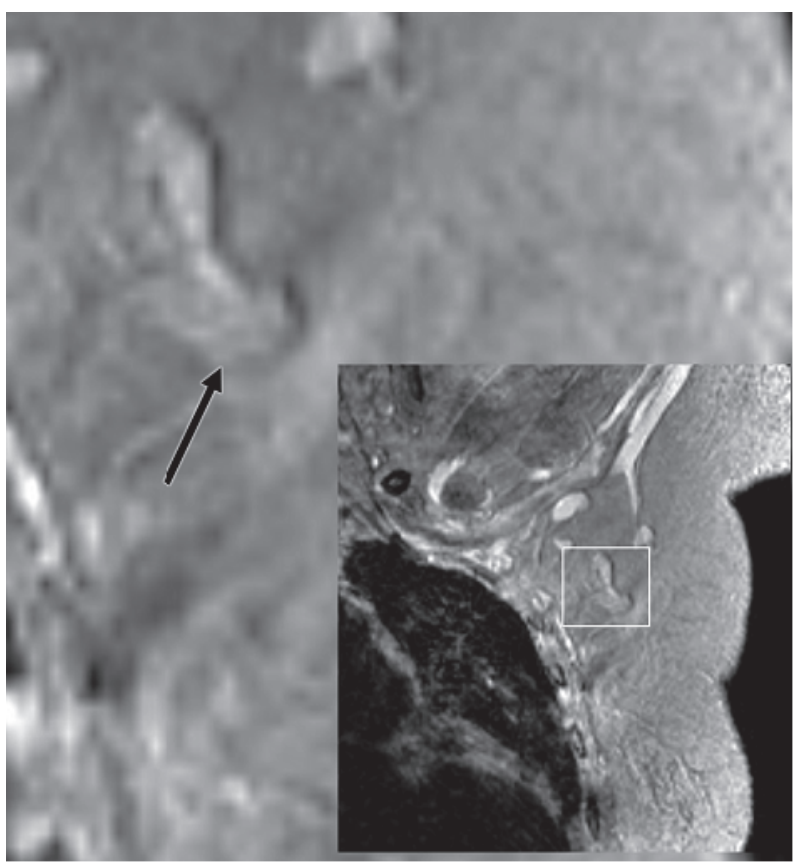

Figure 5.1 MR-images, after completion of neoadjuvant chemotherapy, of a 58-year old women with a 27 $\mathrm{mm}$ large invasive ductal carcinoma in her left breast, which was treated with neoadjuvant chemotherapy followed by lumpectomy with sentinel lymph node biopsy. (A) Coronal T2weighted MR image demonstrates the presence of a fatty hilum in the lymph node. Therefore this lymph node was considered benign by both readers. (B) Coronal T1-weighted MR images after administration of gadofosveset (GDF-MRI) demonstrates the absence of an intact nodal border, which resulted in a malignant lymph node according to one of the two readers. Results of the corresponding histopathology at the sentinel lymph node biopsy revealed a $3.5 \mathrm{~mm}$ macrometastasis of this lymph node.

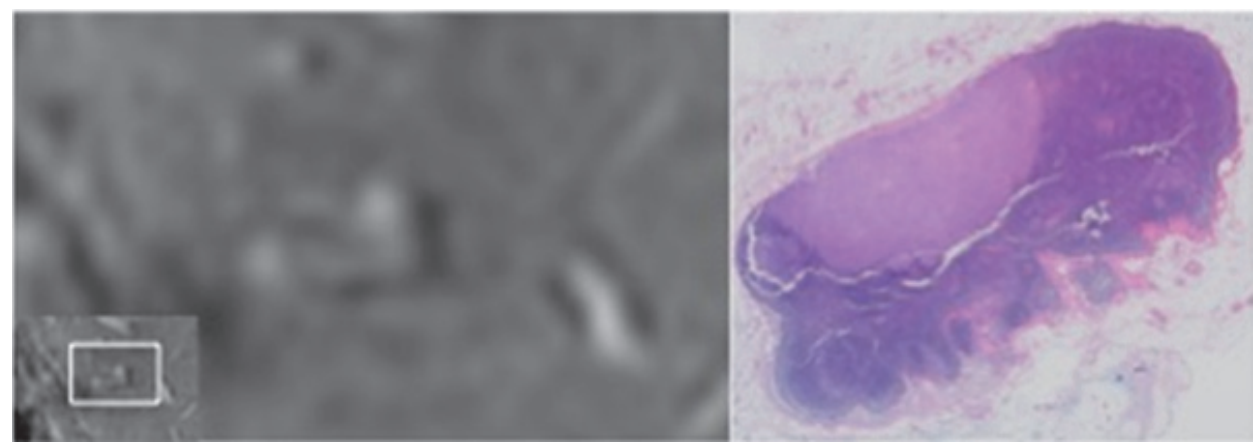

Figure 5.2 An example of a lymph node on coronal T1-weighted GDF-MRI. This node was scored as benign for both readers, based on the presence of a chemical shift artefact and a fatty hilum. However, as seen on the H\&E coupe on the right, the dark area of the lymph node was not a fatty hilum, but was mimicked by a metastatic area. 


\section{Surgery}

The sentinel lymph node was depicted on lymphoscintigraphy by using technetium-99m nanocolloid as radioactive tracer. During surgery, the sentinel node was identified with triple technique consisting of lymphoscintigraphy, blue dye (Bleu Patente V; Guerbet, Aulnay-sous-Bois, France) and detection of radioactivity by gamma probe.

If an (completion) ALND was performed, the following anatomical landmarks were marked: caudal-medial edge, caudal-lateral edge and cranial-lateral edges of the specimen. A clip was placed at the branching of the lateral thoracic vein from the axillary vein. Finally, the medial apex of axilla was marked.

\section{Histopathological evaluation}

For histological evaluation of sentinel nodes, nodes were sliced with a maximum slice thickness of $3 \mathrm{~mm}$ and embedded in paraffin. Each part was step sectioned at 500-micrometer intervals at three levels and stained with haematoxylin and eosin (H\&E). Cytokeratin immunohistochemical staining, using a mouse anti-human MNF116 antibody (Dako, Glostrup, Denmark), was performed if conventional H\&E slices were negative.

For histological evaluation in case of an (completion) ALND, the ALND specimen was received immediately from the operating room. Before formalin fixation, the specimen was fixed in a box representing the normal anatomical situation in the patient. All lymph nodes were embedded in paraffin after 24 hours of fixation. Lymph nodes bigger than $5 \mathrm{~mm}$ were sliced with a maximum slice thickness of $3 \mathrm{~mm}$, and stained with H\&E. ${ }^{14,15}$

Each node was recorded as either benign ( $\mathrm{pNo}$ ) or as isolated tumour cells (ITC) $\quad(\mathrm{pNo}(\mathrm{i}+)) \quad(\leq 0.2 \mathrm{~mm})$, micrometastasis $\quad(\mathrm{pN} 1 \mathrm{mi}) \quad(0.2 \leq 2.0 \mathrm{~mm})$ or macrometastasis $\left(\mathrm{pN}_{1-3}\right) \quad(>2.0 \mathrm{~mm})$. Isolated tumour cells (ITC) and micrometastases were considered negative.

\section{Node-by-node evaluation}

A preoperative SPECT/CT scan was used for matching sentinel nodes with axillary MRI, if available. To achieve a reliable node-by-node match between the ALND specimen and MRI, every node harvested by the pathologist was drawn on an anatomical map. This matching procedure was described in detail in earlier publications. ${ }^{11,15}$ The matching of SPECT/CT images and ALND specimens with axillary MR images was performed by two researchers (T.v.N. and R.J.S.) in consensus; both were blinded to pathology findings and T2W-MRI and GDF-MRI results. 


\section{Statistical analyses}

Statistical analyses were performed using SPSS (version 22.0; IBM, Armonk, New York, USA) and R Project (version 3.3.1; R Foundation for Statistical Computing, Vienna, Austria).

In patients treated with SLNB, lymph nodes on axillary MRI identified as sentinel nodes were taken into account for patient-by-patient analysis only. In patients treated with ALND, all lymph nodes depicted on axillary MRI were included into patient-by-patient analysis.

Diagnostic performance parameters, including sensitivity, specificity, positive predictive value (PPV), negative predictive value (NPV) and area under the receiver operating characteristic (ROC) curve (AUC), were analyzed for T2W-MRI and T2W- + GDF-MRI. The cut-off on the confidence level scale was defined between 2 (possibly benign) and 3 (probably malignant), with lymph nodes scored 2 or lower categorized as benign and those scored 3 or higher as malignant.

Since lymph nodes are clustered per patient when reading axillary MRI, lymph node observations within one patient cannot be regarded as independent observations. To adjust for this within-patient correlation, bootstrapping was performed ( $\mathrm{n}=1000$ repetitions) for calculation of the $95 \%$ confidence intervals for the diagnostic performance parameters. ${ }^{15,16}$

Differences in diagnostic performance parameters between T2W-MRI and $\mathrm{T}_{2} \mathrm{~W}-$ + GDF-MRI of included patients were analyzed. For patient-by-patient validation a nonparametric test for comparing correlated receiver operating characteristic curves was used. ${ }^{17}$

Sample size was based on a previous pilot study investigating GDF-MRI in breast cancer patients. ${ }^{11}$ With an expected sensitivity of $87 \%$ (width of the $95 \%$ confidence interval of $9 \%$ ), a total of 215 lymph nodes with positive test results was required to validate the use of gadofosveset. Based on information of patients treated in our hospital, an average of 1.5 positive lymph nodes was detected in breast cancer patients, resulting in 145 patients. With an expected $10 \%$ drop-out, a total of 160 patients were required.

To investigate the presence of a learning curve for reading GDF-MRI, all diagnostic performance parameters of the first one-third nodes was compared to the next and last one-third, on a node-by-node basis.

$P$-values $<0.05$ were considered statistical significant. 


\section{Results}

\section{Patients}

During the study period, 97 patients were included and underwent $\mathrm{T}_{2} \mathrm{~W}$ - and GDF-MRI. The study was closed early, because the license of manufacturing gadofosveset in the Netherlands was withdrawn. In all patients who underwent GDF-MRI, no serious adverse events were observed. Seven patients were excluded; one because of withdrawal after signing informed consent, two because of claustrophobia during axillary MRI, one since no axillary surgery was performed, one because histopathology showed chronic lymphocytic leukaemia, one because of silicon adenitis in the lymph nodes, and one because of technical interruption concerning the storage of the MR images. Consequently, results of 90 patients were included in final analysis. Patient and tumour characteristics are summarized in Table 5.1. Seventy-eight patients underwent SLNB or ALND first; the remaining twelve were treated with neoadjuvant systemic therapy. Median time interval between axillary MRI and surgery was seven days (range o-3o days).

Table 5.1 Patient demographics and tumour characteristics.

\begin{tabular}{|c|c|}
\hline Characteristic & Value \\
\hline Number of Patients & 90 \\
\hline \multicolumn{2}{|l|}{ Age, years } \\
\hline Mean (SD) & $59(11)$ \\
\hline Range & $22-80$ \\
\hline \multicolumn{2}{|c|}{ Clinical tumour size in situ carcinoma, $\mathrm{mm}(\mathrm{n}=6)$} \\
\hline Mean $(\mathrm{SD})$ & $37(25)$ \\
\hline Range & $17-85$ \\
\hline \multicolumn{2}{|c|}{ Clinical tumour size invasive carcinoma, $\mathrm{mm}(\mathrm{n}=84)$} \\
\hline Mean $(\mathrm{SD})$ & $21(11)$ \\
\hline Range & $6-64$ \\
\hline \multicolumn{2}{|l|}{ cT-stadium, n (\%) } \\
\hline Tis & $6(7)$ \\
\hline $\mathrm{T} 1$ & $48(53)$ \\
\hline $\mathrm{T}_{2}$ & $33(37)$ \\
\hline $\mathrm{T}_{3}$ & $3(3)$ \\
\hline \multicolumn{2}{|l|}{ pN-stadium, n (\%) } \\
\hline pNo & $57(64)$ \\
\hline $\mathrm{pNo}(\mathrm{i}+)$ & $5(6)$ \\
\hline pNımi & $5(6)$ \\
\hline $\mathrm{pN}_{1}$ & $9(10)$ \\
\hline $\mathrm{pN}_{2}$ & $4(4)$ \\
\hline $\mathrm{pN}_{3}$ & $1(1)$ \\
\hline ypNo & $4(4)$ \\
\hline ypNimi & $1(1)$ \\
\hline ypN1 & $1(1)$ \\
\hline $\mathrm{ypN}_{2}$ & $1(1)$ \\
\hline $\mathrm{ypN}_{3}$ & $2(2)$ \\
\hline
\end{tabular}


Table 5.1 (continued)

\begin{tabular}{lc}
\hline Characteristic & Value \\
Pathologic tumour type, $\mathrm{n}(\%)$ & $6(7)$ \\
Ductal carcinoma in situ & $65(72)$ \\
Ductal & $13(14)$ \\
Lobular & $6(7)$ \\
Other & \\
Uni-/multifocal tumours n (\%) & $72(80)$ \\
Unifocal & $18(20)$ \\
Multifocal and/or multicentric & \\
Hormone receptor and HER2 status, n (\%) & $11(12)$ \\
Triple negative & $66(73)$ \\
ER/PR + HER2 - & $1(1)$ \\
ER/PR - HER2 + & $6(7)$ \\
ER/PR + HER2 + & $6(7)$ \\
Not determined (in case of in situ carcinoma) & \\
\hline
\end{tabular}

$\mathrm{N}$, number of cases; SD, standard deviation; ER, estrogen receptor; PR, progesterone receptor; HER2, human epidermal growth factor receptor 2.

\section{Histopathology}

A total of 362 lymph nodes were removed during SLNB (147 nodes) and ALND (215 nodes), of which 263 could be matched to lymph nodes identified on T2W-MRI. Histopathological examination resulted in 75 macrometastases and 188 negative lymph nodes (including 6 ITCs and 16 micrometastases). On patient level, at least one axillary macrometastasis was detected in 20 out of 90 patients. Findings on axillary MRI compared to histopathology are presented in Table 5.2.

Table 5.2 Lymph node scoring on axillary MRI and findings by histopathology.

\begin{tabular}{|c|c|c|c|c|c|c|}
\hline & & & \multicolumn{4}{|c|}{ Histopathology } \\
\hline & & & Benign & Isolated tumour cells & Micrometastasis & Macrometastasis \\
\hline \multirow[t]{4}{*}{ R1 } & T2W-MRI & - & 149 & 6 & 14 & 36 \\
\hline & & + & 17 & o & 2 & 39 \\
\hline & T2W- + GDF-MRI & - & 146 & 6 & 12 & 31 \\
\hline & & + & 20 & o & 4 & 44 \\
\hline \multirow[t]{4}{*}{$\mathrm{R}_{2}$} & T2W-MRI & - & 157 & 6 & 16 & 43 \\
\hline & & + & 9 & o & o & 32 \\
\hline & $\mathrm{T}_{2} \mathrm{~W}-+$ GDF-MRI & - & 152 & 6 & 14 & 38 \\
\hline & & + & 14 & o & 2 & 37 \\
\hline
\end{tabular}

R1, radiologist 1; R2, radiologist 2; T2W-MRI = T2-weighted MRI; GDF-MRI, gadofosveset-enhanced T1weighted MRI. Absolute numbers are presented.

\section{Diagnostic performance: patient-by-patient analysis}

For reader 1 AUC-values of T2W-MRI and T2W-MRI + GDF-MRI were 0.75 and $0.77(P=0.731)$, for reader 20.79 and $0.72(P=0.156)$. Corresponding diagnostic parameters and 95\% confidence intervals (CIs) are presented in Table 5.3. 


\section{Diagnostic performance: node-by-node analysis}

For reader 1, T2W-MRI showed an AUC of 0.76 and T2W-MRI + GDF-MRI an AUC of $0.82(P=0.018)$. For reader 2 AUC of both T2W-MRI and T2W-MRI + GDF-MRI was $0.77(P=0.997)$, (Table 5.3).

Table 5.3 Diagnostic performance for prediction of axillary nodal status.

\begin{tabular}{|c|c|c|c|c|}
\hline & \multicolumn{2}{|c|}{ R1 } & \multicolumn{2}{|c|}{$\mathrm{R}_{2}$} \\
\hline & T2W-MRI & T2W- + GDF-MRI & T2W-MRI & T2W- + GDF-MRI \\
\hline \multicolumn{5}{|c|}{ Node-by-node (prevalence of metastasis: $75 / 263$ ) } \\
\hline Sensitivity & $52(39 / 75)$ & $59(44 / 75)$ & $43(32 / 75)$ & $49(37 / 75)$ \\
\hline $95 \% \mathrm{CI}$ & $40-63$ & $48-69$ & $31-55$ & $39-60$ \\
\hline Specificity & $90(169 / 188)$ & $87(164 / 188)$ & $95(179 / 188)$ & $92(172 / 188)$ \\
\hline $95 \% \mathrm{CI}$ & 86-94 & $82-91$ & $92-98$ & 88-95 \\
\hline PPV & $67(39 / 58)$ & $65(44 / 68)$ & $78(32 / 41)$ & $70(37 / 53)$ \\
\hline $95 \%$ CI & $57-78$ & $56-74$ & $66-89$ & $59-80$ \\
\hline NPV & $82(169 / 205)$ & $84(164 / 195)$ & $81(179 / 222)$ & $82(172 / 210)$ \\
\hline $95 \% \mathrm{CI}$ & $79-86$ & $81-88$ & $77-84$ & $79-85$ \\
\hline AUC & 0.76 & 0.82 & 0.77 & 0.77 \\
\hline $95 \%$ CI & $0.69-0.83$ & $0.76-0.87$ & $0.70-0.84$ & $0.71-0.83$ \\
\hline$P$ Value $^{\dagger}$ & \multicolumn{2}{|c|}{0.018} & \multicolumn{2}{|c|}{0.998} \\
\hline \multicolumn{5}{|c|}{ Patient-by-patient (prevalence of metastasis: $20 / 90$ ) } \\
\hline Sensitivity & $40(8 / 20)$ & $40(8 / 20)$ & $30(6 / 20)$ & $35(7 / 20)$ \\
\hline $95 \% \mathrm{CI}$ & $20-64$ & $20-64$ & $13-54$ & $16-59$ \\
\hline Specificity & $90(63 / 70)$ & $84(59 / 70)$ & $94(66 / 70)$ & $91(64 / 70)$ \\
\hline $95 \%$ CI & $80-96$ & $73-92$ & $85-98$ & $82-96$ \\
\hline PPV & $53(8 / 15)$ & $42(8 / 19)$ & $60(6 / 10)$ & $54(7 / 13)$ \\
\hline $95 \%$ CI & $27-78$ & $21-66$ & $27-86$ & $26-80$ \\
\hline NPV & $84(63 / 75)$ & $83(59 / 71)$ & $83(66 / 80)$ & $83(64 / 77)$ \\
\hline $95 \% \mathrm{CI}$ & $73-91$ & $72-91$ & $72-90$ & $72-90$ \\
\hline AUC & 0.75 & 0.77 & 0.79 & 0.72 \\
\hline $95 \%$ CI & $0.62-0.88$ & $0.66-0.88$ & $0.67-0.90$ & $0.58-0.86$ \\
\hline$P$ Value $^{\ddagger}$ & \multicolumn{2}{|c|}{0.731} & \multicolumn{2}{|c|}{0.156} \\
\hline
\end{tabular}

R1, radiologist 1; $\mathrm{R}_{2}$, radiologist 2; T2W-MRI = T2-weighted MRI; GDF-MRI, gadofosveset-enhanced T1weighted MRI; 95\% CI, 95\% confidence interval; PPV, positive predictive value; NPV, negative predictive value; AUC, area under the curve; numbers are percentages, absolute numbers are given in parentheses.

${ }^{\dagger} P$ Value for difference in AUC between T2W- and GDF-MRI, based on bootstrap test (n=10oo). ${ }^{\ddagger} P$ Value for difference in AUC between T2W- and GDF-MRI, based on DeLong's test.

\section{Learning curve analysis}

For reader 1, AUC of T2W-MRI + GDF-MRI of the first 88 nodes was 0.71 , while the AUC of the following 88 nodes was 0.80 and the AUC of the last 87 nodes was 0.95. The AUC-values of reader 2 were $0.69,0.80$ and 0.79 . Corresponding ROC curves of the AUC are presented in Figure 5.3, and all corresponding diagnostic parameters are presented in Table 5.4. 

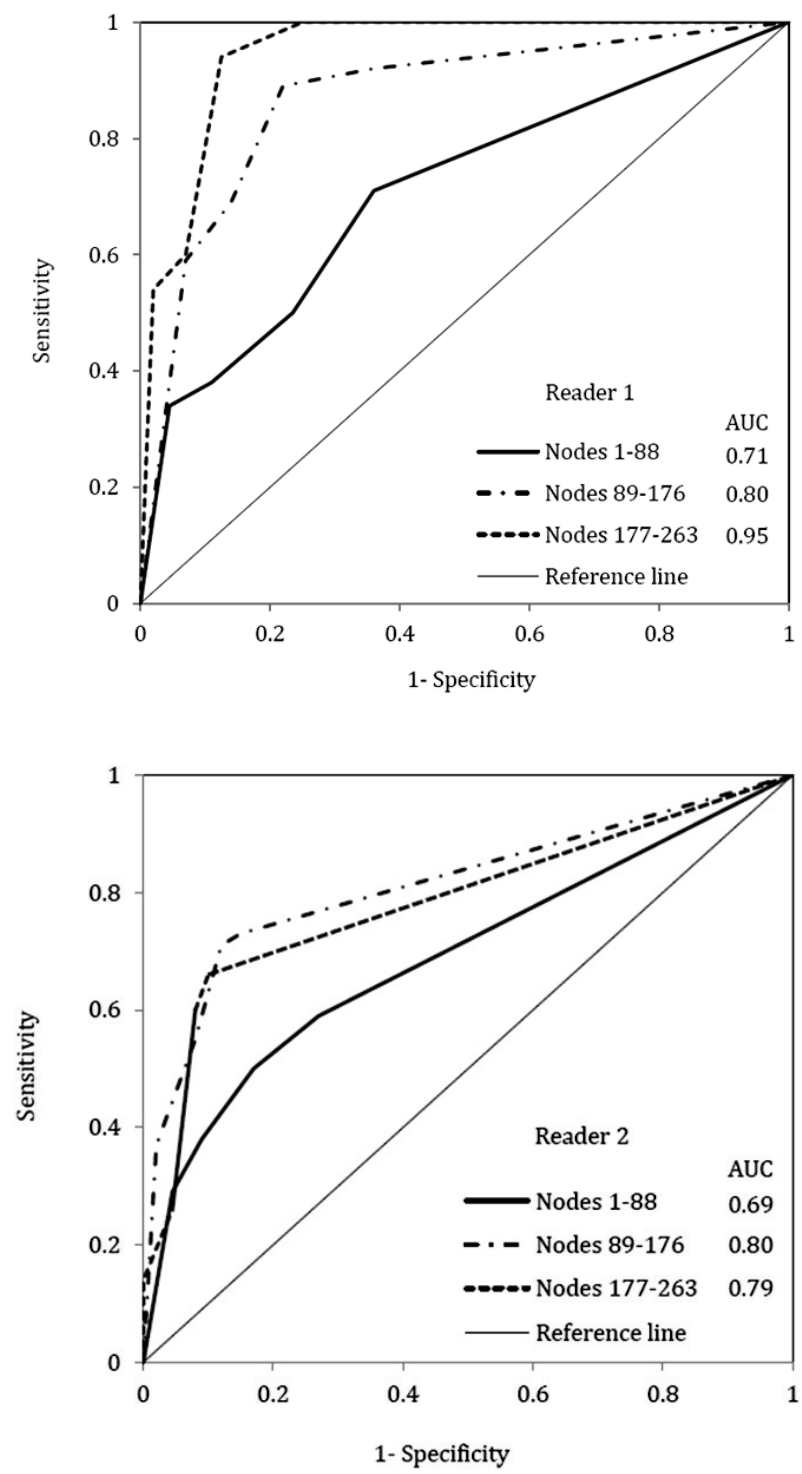

Figure 5.3 Receiver operating characteristics curves and areas under the curve (AUC) based on node-bynode analysis for detection of metastatic lymph nodes in the first 88 (bold line), next 88 (dash dotted line) and last 87 (dotted line) nodes, using the combination of T2-weighted (T2W) and gadofosveset-enhanced T1-weighted (GDF) MRI for reader 1 (a) and 2 (b). 
Table 5.4 Diagnostic performance for prediction of axillary nodal status node-by-node

\begin{tabular}{lcccccc}
\hline & \multicolumn{3}{c}{ R1 T2W- + GDF-MRI } & \multicolumn{3}{c}{ R2 T2W + GDF-MRI } \\
\cline { 2 - 6 } & Nodes & Nodes & Nodes & Nodes & Nodes & Nodes \\
& $1-88$ & $89-176$ & $177-263$ & $1-88$ & $89-176$ & $177-263$ \\
\hline Sensitivity & 38 & 72 & 60 & 38 & 67 & 27 \\
& $(9 / 24)$ & $(26 / 36)$ & $(9 / 15)$ & $(9 / 24)$ & $(24 / 36)$ & $(4 / 15)$ \\
95\% CI & $21-58$ & $58-86$ & $33-87$ & $21-54$ & $50-81$ & $7-47$ \\
Specificity & 89 & 77 & 93 & 91 & 87 & 96 \\
& $(57 / 64)$ & $(40 / 52)$ & $(67 / 72)$ & $(58 / 64)$ & $(45 / 52)$ & $(69 / 72)$ \\
$95 \%$ CI & $81-95$ & $65-88$ & $86-99$ & $83-97$ & $77-94$ & $90-100$ \\
PPV & 56 & 68 & 64 & 60 & 77 & 57 \\
& $(9 / 16)$ & $(26 / 38)$ & $(9 / 14)$ & $(9 / 15)$ & $(24 / 31)$ & $(4 / 7)$ \\
$95 \%$ CI & $35-79$ & $58-81$ & $44-88$ & $37-85$ & $66-90$ & $17-100$ \\
NPV & 79 & 80 & 92 & 79 & 79 & 86 \\
& $(57 / 72)$ & $(40 / 50)$ & $(67 / 73)$ & $(58 / 73)$ & $(45 / 57)$ & $(69 / 80)$ \\
$95 \%$ CI & $74-85$ & $72-89$ & $87-97$ & $74-85$ & $71-87$ & $83-90$ \\
AUC & 0.71 & 0.80 & 0.95 & 0.69 & 0.80 & 0.79 \\
$95 \%$ CI & $0.58-0.82$ & $0.70-0.88$ & $0.90-0.98$ & $0.56-0.80$ & $0.71-0.89$ & $0.66-0.92$ \\
\hline
\end{tabular}

$\mathrm{R}_{1}$, radiologist 1; T2W-MRI = T2-weighted MRI; GDF-MRI, gadofosveset-enhanced T1-weighted MRI; R2, radiologist 2; 95\% CI, 95\% confidence interval; PPV, positive predictive value; NPV, negative predictive value; AUC, area under the curve; numbers are percentages, absolute numbers are given in parentheses.

\section{Discussion}

The aim of this prospective study was to evaluate the diagnostic performance of gadofosveset-enhanced MRI for nodal (re)staging in breast cancer. Both readers showed comparable diagnostic performance of T2W-MRI (per node AUC of 0.76 and 0.77 ). The addition of GDF-MRI improved the performance for reader 1 to $0.82(P=0.018)$, while reader 2 had no benefit of GDF-MRI compared to T2W-MRI.

To understand whether a learning curve existed for GDF-MRI, both readers had feedback on their errors after each axillary MRI. The reader who had the longest experience in nodal staging with GDF-MRI (reader 1) improved in performance after addition of GDF-MRI to T2W-MRI, from an AUC of 0.71 for the first 88 nodes to 0.95 for the last 87 nodes. The breast imaging specialized radiologist (reader 2) did not seem to improve in performance. This suggests that an individual learning curve exists.

In this study, two radiologists with different levels of experience in breast cancer imaging and nodal staging participated. The diagnostic performances of T2W-MRI for both expert (reader 2) and non-expert (reader 1) in breast imaging are in line with previous reports on nodal staging in breast cancer with standard T2W-MR sequences. ${ }^{18}$ This suggests that radiologists - whether or not specialized in breast cancer imaging, do well with nodal staging at standard T2W-MRI. Yet, this remains insufficiently accurate to replace SLNB, with reported NPVs of $84 \%$ or lower in our study. 
Of interest is the difference in performance using the combined T2W-MRI + GDF-MRI of the two radiologists after the first 176 nodes. In the last 87 nodes of our cohort, reader 1 showed a substantial improvement after addition of GDF-MRI to T2W-MRI, from an AUC of 0.71 to 0.95 , equal to reported figures of rectal cancer MR nodal staging using GDF-MRI. ${ }^{19,20}$ Reader 2 however, did not seem to improve. A possible explanation might be that reader 1 was inexperienced in breast imaging interpretation, but had extensive experience in rectal cancer nodal staging with MRI, including 8 years of interpreting gadofosveset-enhanced MRIs. Therefore, reader 1 was more aware of interpretation difficulties with GDF-MRI and of own pitfalls, because of previous experience and continuous feedback in rectal cancer. ${ }^{19}$ Although reader 2 is an expert breast radiologist, he was not familiar with GDF-MRI, which might explain differences in performances.

The diagnostic performance of T2W-MRI and T2W-MRI + GDF-MRI was calculated based on histopathology of surgically removed lymph nodes. Lymph nodes containing ITCs and micrometastases were recorded as benign findings. However, the clinical relevance of these small metastases is limited. ${ }^{21-25}$ Therefore, ignoring micrometastases and ITCs is justified.

Axillary ultrasound combined with ultrasound-guided biopsy if indicated, shows high specificity (98.3\%) and high PPV (97.1\%). ${ }^{26}$ The PPV of GDF-MRI cannot compete with that of ultrasound-guided biopsy, as it is not higher than $78 \%$. Therefore, our study showed that GDF-MRI cannot replace ultrasoundguided nodal biopsy to confirm positive lymph nodes.

Of special interest is the NPV of $92 \%$ of GDF-MRI, suggesting that GDF-MRI can identify (y)pNo patients. This NPV of GDF-MRI outperforms the NPV of axillary ultrasound. ${ }^{26-28}$ Furthermore, an NPV of $92 \%$ approximates the NPV of $96 \%$ of the more invasive SLNB. ${ }^{29}$ It also outperforms the diagnostic performance of SLNB post-neoadjuvant systemic therapy or the MARI-procedure in clinically node positive breast cancer patients. ${ }^{30-32}$ Unfortunately, due to the relatively low sensitivity of GDF-MRI (27-60\%) it will remain challenging for implementation of GDF-MRI into clinical practice.

This study is limited by the relatively small number of patients. According to sample size calculation, a total of 215 positive lymph nodes with an expected sensitivity of $87 \%$ was required. However, in this study we only included a total of 75 positive lymph nodes, since this study was closed early. Furthermore, the difference in sensitivity between a previous pilot study and this study for reading GDF-MRI (86\% versus 59\%) can be explained by different inclusion criterion: clinically palpable nodes or suspicious nodes with axillary ultrasound in the pilot study versus breast cancer patients without any information for the readers 
concerning nodal status in this study. Next, the results of this study are highly dependent on reader's performance. Consequently, reproducibility of these results by less experienced readers could be challenging. Finally, predictive values (PPV and NPV) for investigating T2W- and GDF-MRI depend on prevalence of axillary macrometastases in this study. Therefore, results should be interpreted with the prevalences of this cohort in mind, which consisted of $28.5 \%$ (node-by-node) and 22.2\% (patient-by-patient).

The current study confirmed previous study results that GDF-MRI, in addition to T2W-MRI, has potential as a noninvasive method for nodal (re)staging in breast cancer. 


\section{References}

Torre LA, Bray F, Siegel RL, Ferlay J, Lortet-Tieulent J, Jemal A. Global cancer statistics, 2012. CA Cancer J Clin 2015;65:87-108.

Howlader N, Noone AM, Krapcho M et al. SEER Cancer Statistics Review, 1975-2010. National Cancer Institute. Available via http://seer.cancer.gov/csr/1975_2010/. Accessed 05-05 2017. 2013.

Sackey H, Magnuson A, Sandelin K et al. Arm lymphoedema after axillary surgery in women with invasive breast cancer. Br J Surg 2014;101:390-7.

Schijven MP, Vingerhoets AJ, Rutten HJ et al. Comparison of morbidity between axillary lymph node dissection and sentinel node biopsy. Eur J Surg Oncol 2003;29:341-350

Ashikaga T, Krag DN, Land SR et al (2010) Morbidity results from the NSABP B-32 trial comparing sentinel lymph node dissection versus axillary dissection. J Surg Oncol 2010;102:111-8.

Krag DN, Anderson SJ, Julian TB et al. Sentinel-lymph-node resection compared with conventional axillary-lymph-node dissection in clinically node-negative patients with breast cancer: overall survival findings from the NSABP B-32 randomised phase 3 trial. Lancet Oncol 2010;11:927-33.

DiSipio T, Rye S, Newman B, Hayes S. Incidence of unilateral arm lymphoedema after breast cancer: a systematic review and meta-analysis. Lancet Oncol 2013;14:500-15.

Lucci A, McCall LM, Beitsch PD et al. Surgical complications associated with sentinel lymph node dissection (SLND) plus axillary lymph node dissection compared with SLND alone in the American College of Surgeons Oncology Group Trial Zoo11. J Clin Oncol 2007;25:3657-63.

Voogd AC, Coebergh JW, Repelaer van Driel OJ et al. The risk of nodal metastases in breast cancer patients with clinically negative lymph nodes: a population-based analysis. Breast Cancer Res Treat 2000;62:63-9.

van Nijnatten TJ, Schipper RJ, Lobbes MB, Nelemans PJ, Beets-Tan RG, Smidt ML. The diagnostic performance of sentinel lymph node biopsy in pathologically confirmed node positive breast cancer patients after neoadjuvant systemic therapy: A systematic review and meta-analysis. Eur J Surg Oncol 2015;41:1278-87.

Schipper RJ, Smidt ML, van Roozendaal LM et al. Noninvasive nodal staging in patients with breast cancer using gadofosveset-enhanced magnetic resonance imaging: a feasibility study. Invest Radiol 2013;48:134-9.

Baltzer PA, Dietzel M, Burmeister HP et al. Application of MR mammography beyond local staging: is there a potential to accurately assess axillary lymph nodes? evaluation of an extended protocol in an initial prospective study. AJR Am J Roentgenol 2011;196:W641-7.

Lambregts DM, Heijnen LA, Maas M et al. Gadofosveset-enhanced MRI for the assessment of rectal cancer lymph nodes: predictive criteria. Abdom Imaging 2013;38:720-7.

Lester SC, Bose S, Chen YY et al. Protocol for the examination of specimens from patients with invasive carcinoma of the breast. Arch Pathol Lab Med 2009;133:1515-38.

Schipper RJ, Paiman ML, Beets-Tan RG et al. Diagnostic Performance of Dedicated Axillary T2and Diffusion-weighted MR Imaging for Nodal Staging in Breast Cancer. Radiology 2015;275: 345-55.

Efron B. Bootstrap Methods: Another Look at the Jackknife. Ann Statist 1979;7:1-26.

DeLong ER, DeLong DM, Clarke-Pearson DL. Comparing the areas under two or more correlated receiver operating characteristic curves: a nonparametric approach. Biometrics 1988;44:837-45.

Kuijs VJ, Moossdorff M, Schipper RJ et al. The role of MRI in axillary lymph node imaging in breast cancer patients: a systematic review. Insights Imaging 2015;6:203-15.

Lambregts DM, Beets GL, Maas M et al. Accuracy of gadofosveset-enhanced MRI for nodal staging and restaging in rectal cancer. Ann Surg 2011;253:539-45.

Heijnen LA, Lambregts DM, Martens MH et al. Performance of gadofosveset-enhanced MRI for staging rectal cancer nodes: can the initial promising results be reproduced? Eur Radiol 2014;24(2):371-9. 
21 Galimberti V, Cole BF, Zurrida S et al. Axillary dissection versus no axillary dissection in patients with sentinel-node micrometastases (IBCSG 23-01): a phase 3 randomised controlled trial. Lancet Oncol 2013;14:297-305.

22 Giuliano AE, Ballman K, McCall L et al. Locoregional Recurrence After Sentinel Lymph Node Dissection With or Without Axillary Dissection in Patients With Sentinel Lymph Node Metastases: Long-term Follow-up From the American College of Surgeons Oncology Group (Alliance) ACOSOG Zoon Randomized Trial. Ann Surg 2016;264:413-20.

23 van Roozendaal LM, Schipper RJ, Van de Vijver KK et al. The impact of the pathological lymph node status on adjuvant systemic treatment recommendations in clinically node negative breast cancer patients. Breast Cancer Res Treat 2014;143:469-76.

24 de Boer M, van Deurzen CH, van Dijck JA et al. Micrometastases or isolated tumor cells and the outcome of breast cancer. N Engl J Med 2009;361:653-63.

25 Maaskant-Braat AJ, van de Poll-Franse LV, Voogd AC et al. Sentinel node micrometastases in breast cancer do not affect prognosis: a population-based study. Breast Cancer Res Treat 2011;127:195-203.

26 Houssami N, Ciatto S, Turner RM, Cody HS, zrd, Macaskill P. Preoperative ultrasound-guided needle biopsy of axillary nodes in invasive breast cancer: meta-analysis of its accuracy and utility in staging the axilla. Ann Surg 2011;254:243-51.

27 Schipper RJ, van Roozendaal LM, de Vries B et al. Axillary ultrasound for preoperative nodal staging in breast cancer patients: is it of added value? Breast 2013;22:1108-13.

28 Alvarez S, Anorbe E, Alcorta P, Lopez F, Alonso I, Cortes J. Role of sonography in the diagnosis of axillary lymph node metastases in breast cancer: a systematic review. AJR Am J Roentgenol 2006;186:1342-8.

29 Krag DN, Anderson SJ, Julian TB et al. Technical outcomes of sentinel-lymph-node resection and conventional axillary-lymph-node dissection in patients with clinically node-negative breast cancer: results from the NSABP B-32 randomised phase III trial. Lancet Oncol 2007;8:881-8.

30 Boughey JC, Suman VJ, Mittendorf EA et al. Sentinel lymph node surgery after neoadjuvant chemotherapy in patients with node-positive breast cancer: the ACOSOG Z1071 (Alliance) clinical trial. JAMA 2013;310:1455-61.

31 Kuehn T, Bauerfeind I, Fehm T et al. Sentinel-lymph-node biopsy in patients with breast cancer before and after neoadjuvant chemotherapy (SENTINA): a prospective, multicentre cohort study. Lancet Oncol 2013;14:609-18.

32 Donker M, Straver ME, Wesseling J et al. Marking axillary lymph nodes with radioactive iodine seeds for axillary staging after neoadjuvant systemic treatment in breast cancer patients: The MARI Procedure. Ann Surg 2015;261(2):378-82. 



\section{Chapter}

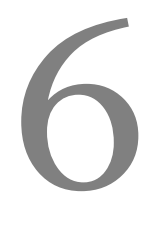

\section{Added value of dedicated axillary hybrid 18F-FDG PET/MRI to improve axillary nodal staging in clinically node positive breast cancer patients - a feasibility study}

T.J.A. van Nijnatten, B. Goorts, S. Vöö, M. de Boer, L.F.S. Kooreman, E.M. Heuts, J.E. Wildberger, F.M. Mottaghy, M.B.I. Lobbes, M.L. Smidt Eur J Nuc Med Mol Imaging. 2017 [Epub ahead of print] 


\section{Abstract}

\section{Objective}

To investigate the feasibility and potential added value of dedicated axillary 18FFDG hybrid PET/MRI, compared to standard imaging modalities (i.e. ultrasound (US), MRI and PET/CT) for axillary nodal staging in clinically node-positive breast cancer.

\section{Methods}

Twelve patients with clinically node-positive breast cancer underwent axillary US and dedicated axillary hybrid 18F-FDG PET/MRI. Nine out of these twelve patients also underwent whole-body PET/CT. Maximum standardized uptake values (SUVmax) were measured for the primary breast tumour and the most FDG-avid axillary lymph node. A positive axillary lymph node on dedicated axillary hybrid PET/MRI was defined as a moderate to very intense FDG-avid lymph node. The diagnostic performance of dedicated axillary hybrid PET/MRI was calculated by comparing quantitative and its qualitative measurements to results of axillary US, MRI and PET/CT. The number of suspicious axillary lymph nodes was subdivided as follows: No (o nodes), $\mathrm{N}_{1}$ (1-3 nodes), $\mathrm{N}_{2}$ (4-9 nodes) and $\mathrm{N}_{3}$ ( $\geq 10$ nodes).

\section{Results}

According to dedicated axillary hybrid PET/MRI findings, seven patients were diagnosed with $\mathrm{N}_{1}$, four $\mathrm{N}_{2}$ and one $\mathrm{N}_{3}$. With regard to mean SUVmax, there was no significant difference of primary tumour (9.0 $( \pm 5.0)$ versus $8.6( \pm 5.7), P=0.678)$ or the most FDG-avid axillary lymph node $(7.8( \pm 5.3)$ versus $7.7( \pm 4.3), P=0.767)$ between dedicated axillary PET/MRI and PET/CT. Compared to standard imaging modalities, dedicated axillary hybrid PET/MRI resulted in changes in nodal status as follows: $40 \%$ compared to US, $75 \%$ compared to T2 weighted MRI, $40 \%$ compared to contrast-enhanced MRI and 22\% compared to PET/CT.

\section{Conclusion}

Adding dedicated axillary 18F-FDG hybrid PET/MRI to diagnostic work-up may improve diagnostic work-up of axillary nodal staging in clinically node-positive breast cancer patients. 


\section{Introduction}

Breast cancer is one of the most prevalent types of invasive cancer among women. ${ }^{1}$ The involvement of axillary lymph nodes is an important prognostic factor, as 5 -year overall survival is reduced from $98 \%$ to $85 \%$ in case of lymph node metastases. ${ }^{2}$ Therefore, European guidelines recommend preoperative axillary imaging to differentiate between patients with and without clinically nodepositive breast cancer. ${ }^{3}$ Accurate lymph node staging is important, as it affects the type of axillary surgery and indications for systemic therapy and radiotherapy fields. ${ }^{4}$

In addition to differentiating between node-negative and node-positive disease, an equally important consideration is the number of suspicious nodes. Current treatment plans are based on the number of suspicious lymph nodes (i.e. one to three versus four or more) according to imaging findings and histopathological confirmation prior to start of therapy. ${ }^{3}$ Preoperative axillary imaging currently involves the use of axillary ultrasound (US) with tissue sampling in case of suspicious nodal findings. Schipper et al. demonstrated that more than $50 \%$ of patients with one to three suspicious lymph nodes on US had four or more lymph node metastases at surgery. ${ }^{5}$ This percentage decreased to 11$15 \%$ when standard breast MRI was used for axillary nodal staging. ${ }^{6}$ In addition, the use of a dedicated axillary MR protocol in place of a standard breast MR protocol further improves diagnostic performance of MRI for nodal staging. ${ }^{7}$ Research suggests that PET/CT has the highest accuracy for preoperative nodal staging: Koolen et al. demonstrated that $98 \%$ of all patients with suspicious axillary lymph nodes on PET/CT would eventually have axillary lymph node metastases after surgery. ${ }^{8}$

Hybrid PET/MRI allows for the simultaneous use of the promising axillary nodal staging techniques PET and MRI. ${ }^{9}$ Therefore, the aim of this study was to investigate the feasibility and potential added value of dedicated axillary 18F-FDG hybrid PET/MRI compared to standard imaging modalities (i.e. axillary US, MRI and PET/CT) for axillary nodal staging in patients with clinically node-positive breast cancer.

\section{Materials and methods}

\section{Setting and patients}

After approval of the study by the local medical ethical committee, a total of twelve patients provided signed informed consent to undergo dedicated axillary hybrid PET/MRI. Inclusion criteria were patients with histopathologically 
confirmed clinically node-positive breast cancer. Exclusion criteria were contraindications for either MRI, including contrast agent gadobutrol, or PET, including radiotracer 18F-fluorodeoxyglucose (18F-FDG). Prior to inclusion, patients underwent axillary US. In addition, all patients were planned for MR imaging and, depending on number of suspicious lymph nodes (i.e. one to three versus four or more), for PET/CT as well. ${ }^{10}$

\section{Axillary ultrasound}

Axillary US exams were performed by the radiologist on call, using an ultrasound system with a linear 2 to $17 \mathrm{MHz}$ array transducer (iU22-xMATRIX, Philips Healthcare, Best, the Netherlands). Criteria for suspicious axillary lymph nodes included diffuse cortical thickening, focal cortical mass and/or thickening and loss of fatty hilum." During axillary US, tissue sampling of suspicious axillary lymph nodes was performed. In cases of multiple suspicious nodes, only the most suspicious node was sampled, and the number of suspicious axillary lymph nodes was reported.

\section{$\mathrm{PET} / \mathrm{CT}$}

In the Netherlands, PET/CT exams can be considered in case of stage III/IV breast cancer. ${ }^{10,12}$ After patients had fasted for a fasting period of at least four hours and had a blood glucose level $<10 \mathrm{mmol} / \mathrm{L}$, an intravenous injection of $18 \mathrm{~F}-\mathrm{FDG}$ was administered with a dose of $2 \mathrm{MBq} / \mathrm{kg}$ bodyweight, followed by a resting period of $60+/-10$ minutes. Patients were placed in supine position with elevated arms in the PET/CT scanner (Gemini TF, Philips Healthcare, Best, the Netherlands). A low-dose CT scan (120 kV, $30 \mathrm{mAs}$, slice thickness $4 \mathrm{~mm}$ ) from head to thigh was performed, followed by the PET acquisition ( 3 minutes per bed position). CT images were reconstructed using filtered back projection. PET images were reconstructed using the BLOB-OS-TF time-of-flight algorithm provided by the manufacturer, with a voxel size of $4 \times 4 \times 4 \mathrm{~mm}^{3}$.

\section{Hybrid PET/MRI}

Directly after whole-body PET/CT, state-of-the-art dedicated axillary hybrid PET/MRI images were acquired on a 3.0T Biograph mMR integrated PET/MRI system (Siemens Healthineers, Erlangen, Germany). Dedicated axillary MRI was performed using a six-channel anterior body coil (Siemens Healthineers), which was placed on the ipsilateral shoulder. Patients were placed in prone position with both arms elevated.

In three cases no whole-body PET/CT was performed. These patients underwent dedicated axillary hybrid PET/MRI after administration of 18F-FDG 
with a dose of $2 \mathrm{MBq} / \mathrm{kg}$ bodyweight and a resting period of $60+/-10$ minutes, with identical fasting time and a blood glucose level $<10 \mathrm{mmol} / \mathrm{L}$ as with PET/CT.

The dedicated axillary hybrid PET/MRI protocol consisted of an unenhanced T2-weighted ( $\left.\mathrm{T}_{2} \mathrm{~W}\right)$ sequence, contrast-enhanced T1-weighted sequence $\left(\mathrm{T}_{1} \mathrm{~W}\right)$ and a fusion sequence of PET-images with the unenhanced $\mathrm{T}_{2} \mathrm{~W}$ sequence. All sequences were performed in coronal view.

More specifically, a $2 \mathrm{D}$ standard $\mathrm{T}_{2}$ weighted $\left(\mathrm{T}_{2} \mathrm{~W}\right)$ sequence without fat suppression was performed first using the following scan parameters: repetition time (TR) $2000 \mathrm{~ms}$; echo time (TE) $118 \mathrm{~ms}$; variable degree flip angle; turbo spin echo (TSE) factor 41; averages 1.4; voxel size $1.1 \times 1.1$ x $1.1 \mathrm{~mm}$; field of view (FOV) $220 \times 220 \mathrm{~mm}^{2}$; matrix $192 \times 192$, resulting in an in-plane resolution of $1.15 \times$ $1.15 \mathrm{~mm}^{2}$. Next, gadobutrol (Gadovist', Bayer HealthCare, Berlin, Germany) contrast with an amount of 15 cc was administered intravenously. After 6 minutes a $3 \mathrm{D}$ T1 weighted $\left(\mathrm{T}_{1} \mathrm{~W}\right)$ sequence was acquired using the following parameters: TR 7.27 ms; TE 2.46 ms; 15 degree flip angle; averages 2; voxel size 1.1 x 1.1 X $1.1 \mathrm{~mm}$; FOV $220 \times 220 \mathrm{~mm}^{2}$; matrix $192 \times 192$; resulting in an in-plane resolution of $1.15 \times$ $1.15 \mathrm{~mm}^{2}$. Total acquisition time of the MRI exam was 20 minutes and 21 seconds.

Three-dimensional iterative reconstruction was performed for all PET images of the hybrid PET/MRI system (number of iterations 3; subsets 21; voxel size 4.1 X $2.6 \times 3.9 \mathrm{~mm}^{3}$; image matrix $172 \times 172$; zoom 1.0) and automatic attenuation correction through implementation of a four-compartment model attenuation map (Dixon based $\mu$-map). Images were acquired in a single bed position within 15 minutes after start of the MRI acquisition on the hybrid PET/MRI system.

\section{Image evaluation}

All axillary lymph nodes on dedicated axillary MR images, hybrid PET/MRI and PET/CT images were evaluated by a breast radiologist (M.B.I.L.) and nuclear medicine physician (S.V.), respectively. The breast radiologist has 7 years of dedicated experience in breast imaging, whereas the nuclear medicine physician has 9 years of experience in nuclear imaging.

First, the radiologist scored each axillary lymph node on dedicated axillary $\mathrm{T} 2 \mathrm{~W}$ images, according to the criteria previously defined by Baltzer er al., using a confidence scale from o (no lymph nodes) to 4 (definitely malignant). ${ }^{13}$ Lymph nodes with a score of o-2 were considered benign, and those with a score of 3-4 malignant. Suspicious characteristics included irregular margins, inhomogeneous cortex, perifocal edema, absent fatty hilum, asymmetry, and absence of chemical shift artifacts. ${ }^{13-15}$ Next, contrast-enhanced dedicated axillary $\mathrm{T}_{1} \mathrm{~W}$ images were shown, which allowed the radiologist to adjust his score on each lymph node, if 
deemed necessary. Suspicious characteristics included absence of contrast hyperintensity of the lymph node and absence of an intact nodal border. ${ }^{15}$

Second, the breast radiologist and nuclear medicine physician evaluated in consensus each axillary lymph node on dedicated axillary hybrid PET/MRI using a four-point system as previously described by Aukema et al, for the assessment of lymph nodes with 18F-FDG PET/CT: o = similar to other surrounding lymph nodes, 1 = slightly more intense than other lymph nodes, $2=$ moderate intense and $3=$ very intense. ${ }^{16}$ Lymph nodes with a score of o-1 FDG uptake were considered benign, while a score of 2-3 FDG uptake was considered malignant. PET information was considered to be decisive regarding the number of suspicious axillary lymph nodes in the evaluation of dedicated axillary hybrid PET/MRI. The nuclear medicine physician measured further maximum standardized uptake values (SUVmax) of the primary breast tumour and the most FDG-avid axillary lymph node on each dedicated axillary hybrid PET/MRI exam. Finally, the nuclear medicine physician evaluated each axillary lymph node on 18F-FDG PET/CT, using the same four-point system, ${ }^{16}$ and measured SUVmax of the primary breast tumour and the most FDG-avid axillary lymph node on each PET/CT exam. Both PET/CT and PET/MRI exams were shown anonymously and in randomized order to the nuclear physician for evaluation of the number of suspicious axillary lymph nodes.

\section{Pathology}

In this study, clinically node-positive disease was based on US-guided cytological and/or histological confirmation of axillary lymph node biopsy. In cases where US was performed in the referring hospital, pathology of the axillary lymph node biopsy was revised and metastases were verified by the pathologist in our hospital (L.K.).

\section{Statistical analyses}

Statistical analyses were performed using SPSS software (version 22, IBM, Armonk, New York, USA). Axillary nodal status was based on number of suspicious axillary lymph nodes: none $(\mathrm{No}), 1-3\left(\mathrm{~N}_{1}\right), 4-9\left(\mathrm{~N}_{2}\right)$ or 10 or more $\left(\mathrm{N}_{3}\right)$, according to axillary US (including results of tissue sampling), dedicated axillary (PET)MRI and/or PET/CT, respectively. When the result of dedicated axillary hybrid PET/MRI led to either up- or downstaging of clinical nodal status compared to standard imaging modalities (US, MRI or PET/CT), it was considered a change in clinical nodal status according to dedicated axillary hybrid PET/MRI findings.

In patients who underwent both PET/CT and hybrid PET/MRI, Wilcoxon signed rank test was used to compare the mean SUVmax of primary tumour 
( \pm standard deviation $(\mathrm{SD})$ ) and the most FDG-avid axillary lymph node $( \pm \mathrm{SD})$ of PET/CT with hybrid PET/MRI. P-values (two-sided) <0.05 were considered statistically significant.

\section{Results}

\section{Patients}

A total of twelve consecutive patients with clinically node-positive breast cancer over a period from April 2016 until March 2017 were included in this feasibility study. The mean age was 49 years (range, $28-70$ years). In all patients, clinically positive nodal status was histopathologically confirmed with US-guided biopsy. Eleven out of twelve patients had invasive carcinoma of no special type, one patient had mixed lobular carcinoma with carcinoma of no special type. In addition, nine out of twelve patients underwent PET/CT. A more detailed overview of the general characteristics is shown in Table 6.1.

Table 6.1 General patient characteristics.

\begin{tabular}{ll}
\hline Mean age (years) (range) & $49(28-70)$ \\
Mean clinical tumour size (mm) (range) & $46(13-150)$ \\
cT-stage (\%) & $2(17 \%)$ \\
cT1 & $4(33 \%)$ \\
cT2 & $3(25 \%)$ \\
cT3 & $3(25 \%)$ \\
cT4 & \\
Multifocal tumour (\%) & $8(67 \%)$ \\
No & $4(33 \%)$ \\
Yes & \\
Tumour type (\%) & $11(92 \%)$ \\
Invasive carcinoma NST & $1(8 \%)$ \\
Mixed ductal and lobular & \\
Tumour grade (\%) & $6(50 \%)$ \\
2 & $6(50 \%)$ \\
3 & \\
Hormonal and receptor status & $4(33 \%)$ \\
ER/PR positive, Her2 negative & $1(8 \%)$ \\
ER/PR negative, Her2 positive & $2(17 \%)$ \\
Triple negative & $5(42 \%)$ \\
ER/PR positive, Her2 positive & \\
\hline
\end{tabular}

Abbreviations: cT-stage clinical tumour stage, NST no special type, ER estrogen, PR progesterone, HER 2 Human Epidermal growth factor Receptor 2. 


\section{Nodal staging}

When compared to standard imaging modalities, dedicated axillary hybrid PET/MRI resulted in changes to clinical nodal status as follows: $40 \%$ according to US findings (4 out of 10 patients), 75\% according to T2W-MRI findings (9 out of 12 patients), $40 \%$ according to CE-T1W MRI findings (4 out of 10 patients) and $22 \%$ according to PET/CT findings (2 out of 9 patients), respectively. Differences between PET/CT and PET/MRI findings were due to better delineation of the lymph nodes on the MRI component. A more detailed overview of all cases, including up- or downstaging of nodal status according to dedicated axillary hybrid PET/MRI findings, is provided in Table 6.2.

In two patients axillary US findings regarding the number of suspicious axillary lymph nodes was missing, because US was performed in the referring hospital. However, in addition, neither patient underwent contrast-enhanced T1-weighted MRI, as this was refused by both patients.

Figures 6.1 and 6.2 illustrate examples of differences in number of suspicious axillary lymph nodes between PET/CT (Figure 6.1) and T2W-MRI (Figure 6.2) when compared to dedicated axillary hybrid PET/MRI. Appendix 1 provides a complete overview of all axillary lymph nodes evaluated on dedicated axillary (PET/)MRI and PET/CT images.

Table 6.2 Clinical nodal status (including number of suspicious lymph nodes) according to US, PET/CT, T2W-MRI, CE-T1W-MRI and hybrid PET/MRI.

\begin{tabular}{|c|c|c|c|c|c|}
\hline \multirow{2}{*}{ Patient } & \multirow{2}{*}{ US } & \multirow{2}{*}{$\mathrm{PET} / \mathrm{CT}$} & \multicolumn{3}{|c|}{ Dedicated axillary (PET/)MRI } \\
\hline & & & $T_{2} W-M R I$ & $C E-T_{1} W M R I$ & Hybrid PET/MRI \\
\hline 1 & $\mathrm{~N} 2(4)$ & N2 (5) & $\mathrm{N}_{3}(10) \downarrow$ & $\mathrm{N}_{3}(10) \downarrow$ & $\mathrm{N}_{2}(8)$ \\
\hline 2 & $\mathrm{~N}_{1}(1)$ & - & No $\uparrow$ & $\mathrm{N}_{1}(\mathbf{1})$ & $\mathrm{N}_{1}(2)$ \\
\hline 3 & $\mathrm{~N}_{1}(1)$ & - & $\mathrm{N}_{2}(5) \downarrow$ & $\mathrm{N}_{2}(7) \downarrow$ & $\mathrm{N}_{1}(1)$ \\
\hline 4 & $\mathrm{~N}_{1}(3) \uparrow$ & N2 (5) & $\mathrm{N}_{3}(10) \downarrow$ & $\mathrm{N}_{2}(9)$ & $\mathrm{N}_{2}(8)$ \\
\hline 5 & $\mathrm{~N}+(-)$ & N2 (9) & $\mathrm{N} 1(3) \uparrow$ & - & N2 (7) \\
\hline 6 & $\mathrm{~N}_{2}(6) \downarrow$ & $\mathrm{N}_{1}(2)$ & $\mathrm{N}_{3}(25) \downarrow$ & $\mathrm{N}_{3}(11) \downarrow$ & $\mathrm{N}_{1}(2)$ \\
\hline 7 & $\mathrm{~N}+(-)$ & $\mathrm{N}_{2}(4) \downarrow$ & $\mathrm{N}_{2}(4) \downarrow$ & - & $\mathrm{N}_{1}(3)$ \\
\hline 8 & $\mathrm{~N}_{1}(1)$ & $\mathrm{N} 1(3)$ & $\mathrm{N}_{2}(6) \downarrow$ & $\mathrm{N}_{2}(7) \downarrow$ & $\mathrm{N} 1(3)$ \\
\hline 9 & $\mathrm{~N}_{2}(5) \uparrow$ & $\mathrm{N} 2(7) \uparrow$ & $\mathrm{N}_{3}(10)$ & $\mathrm{N}_{3}(13)$ & $\mathrm{N}_{3}(10)$ \\
\hline 10 & $\mathrm{~N}_{1}(1)$ & $\mathrm{N}_{1}(1)$ & $\mathrm{N}_{1}(2)$ & $\mathrm{N}_{1}(2)$ & $\mathrm{N}_{1}(1)$ \\
\hline 11 & $\mathrm{~N}_{1}(2) \uparrow$ & - & $\mathrm{N}_{1}(3) \uparrow$ & $\mathrm{N}_{2}(5)$ & $\mathrm{N}_{2}(4)$ \\
\hline 12 & $\mathrm{~N}_{1}(1)$ & $\mathrm{N}_{1}(1)$ & $\mathrm{N}_{1}(2)$ & $\mathrm{N}_{1}(3)$ & $\mathrm{N}_{1}(1)$ \\
\hline
\end{tabular}

Abbreviations: PET Positron Emission Tomography, CT Computed Tomography, MRI Magnetic Resonance Imaging, $T_{2} W$ T2-weighted, $C E-T_{1} W$ contrast-enhanced T1-weighted, No no suspicious lymph nodes, $N_{1}$ 1-3 suspicious lymph nodes, $N_{2}$ 4-9 suspicious lymph nodes, $N_{3}$ 10 or more suspicious lymph nodes, $N+$ clinically node positive status, - missing, $\downarrow$ downstaging according to result of hybrid PET/MRI, $\uparrow$ upstaging according to result of hybrid PET/MRI. 


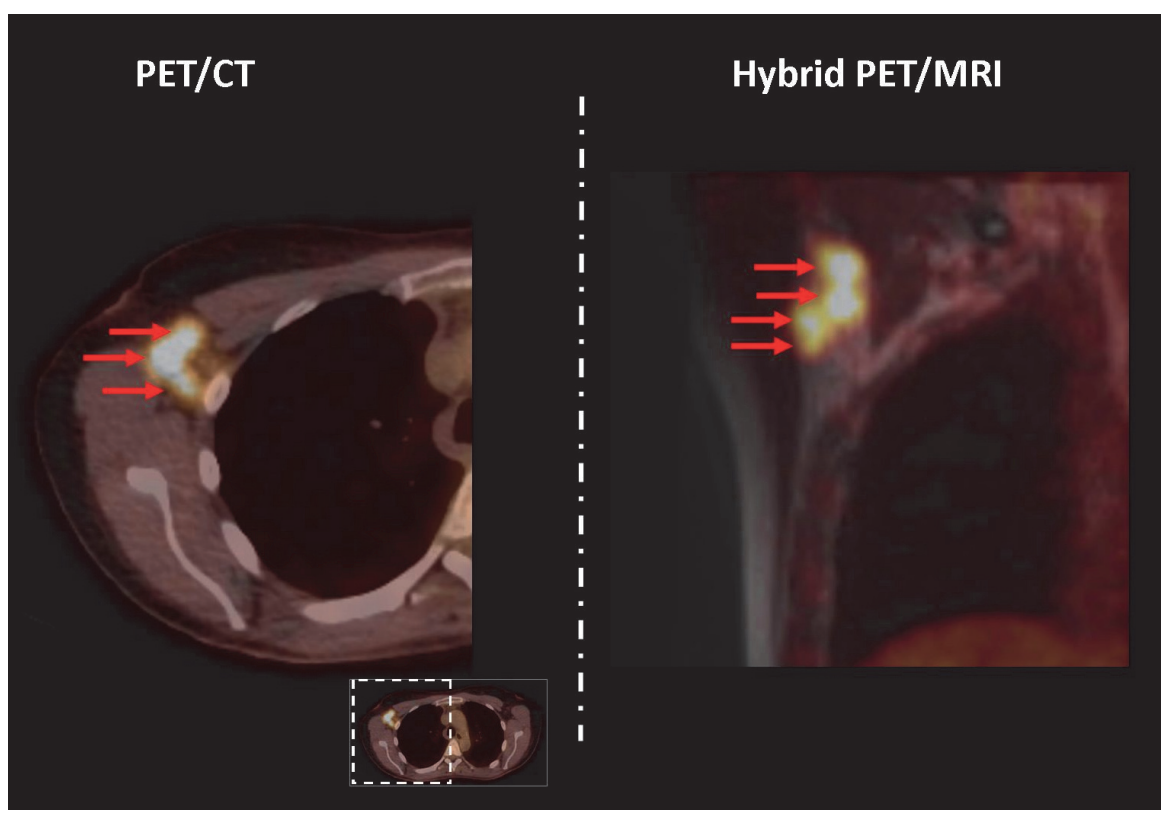

Figure 6.1 Example of a patient with a $35 \mathrm{~mm}$ invasive carcinoma of no special type in her right breast. One cross-sectional PET/CT image (left) of the right axilla demonstrates three suspicious lymph nodes (red arrows) out a total of five suspicious lymph nodes on PET/CT. One coronal dedicated axillary hybrid PET/MRI image (right) demonstrates four suspicious lymph nodes (red arrows), with a total of eight suspicious lymph nodes on dedicated axillary hybrid PET/MRI. The most dorsally located suspicious lymph node on (low-dose) PET/CT consists of two suspicious lymph nodes on dedicated axillary hybrid PET/MRI.

\section{SUVmax measurements}

The mean SUVmax of the primary tumour and the most FDG-avid axillary lymph node on PET/CT were $8.6( \pm 5.7)$ and $7.7( \pm 4.3)$, respectively. Comparable values were achieved on hybrid PET/MRI, with mean SUVmax of primary tumour 9.0 $( \pm 5.0)(P=0.678)$ and mean SUVmax of the most FDG-avid lymph node $7.8( \pm 5.3)$ $(P=0.767)$. Table 6.3 provides an overview of all SUVmax measurements on $\mathrm{PET} / \mathrm{CT}$ and hybrid PET/MRI for each patient. 


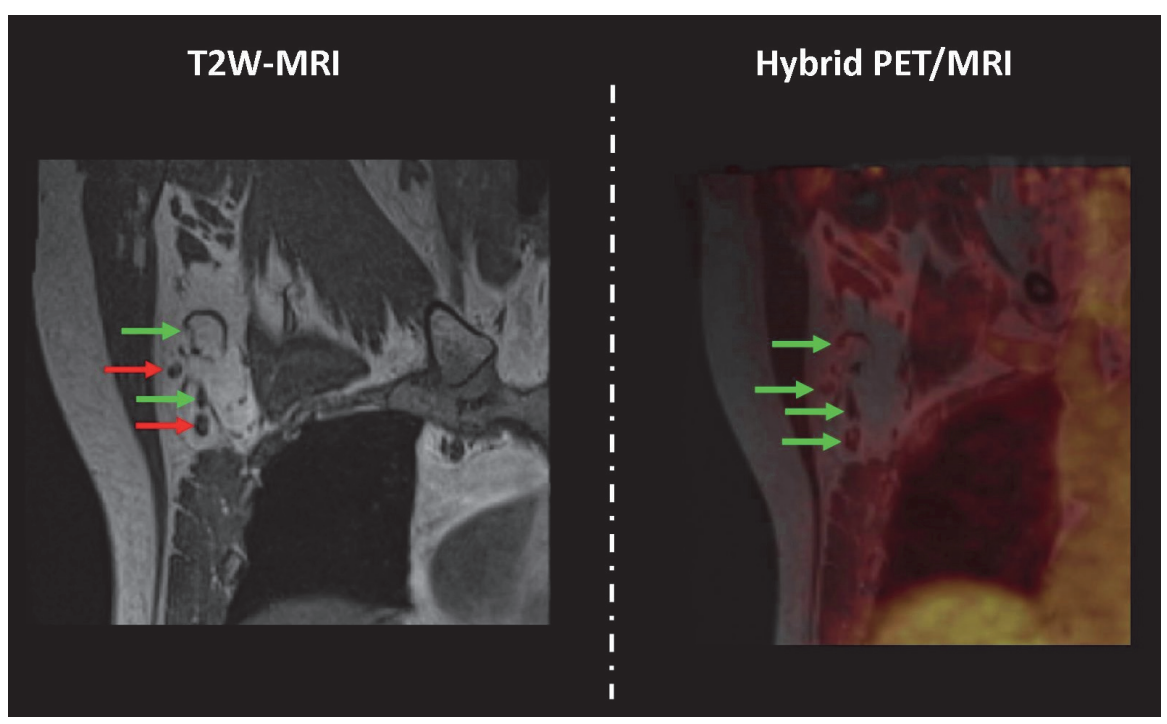

Figure 6.2 Example of a patient with a $31 \mathrm{~mm}$ invasive carcinoma of no special type in her right breast. A coronal T2-weighted MR image (left) of the right axilla demonstrates two out of four (red arrows) suspicious nodes. A coronal dedicated axillary hybrid PET/MRI image (right) demonstrates no FDG-uptake in any of the four nodes, which resulted in four negative nodes on dedicated axillary hybrid PET/MRI.

\section{Discussion}

The aim of this study was to investigate the feasibility and potential added value of dedicated axillary 18F-FDG hybrid PET/MRI compared to standard imaging modalities (i.e. US, MRI and PET/CT) for axillary nodal staging in patients with clinically node-positive breast cancer. The results of our study show that dedicated axillary 18F-FDG hybrid PET/MRI is clinically feasible, and resulted in a change in nodal status in $40-75 \%$ of patients when compared to US or MRI. When compared to PET/CT only, nodal status was changed in two out of nine patients (22\%), although SUVmax measurements were comparable between the imaging modalities.

The importance of accurate differentiation in clinically node-positive patients, i.e. one to three versus four or more positive nodes, prior to start of treatment is a topic of debate since adjuvant (radiation) therapy planning is based on pre-treatment axillary imaging findings in the case of neoadjuvant systemic therapy. ${ }^{17}$ With regard to patients with limited axillary disease after surgery, the 
ACOSOG Zoon trial demonstrated that there was no prognostic benefit in adjuvant axillary treatment in clinically node-negative patients treated with primary surgery. ${ }^{18}$ On the other hand, patients with advanced axillary disease (i.e. four or more positive nodes) do require adjuvant regional treatment. ${ }^{17,19}$ Consequently, pre-treatment axillary imaging is becoming increasingly important as a means of truly identifying patients with four or more versus three or fewer positive nodes. However, long-term outcome for patients treated with additional therapy after the detection of a larger number of positive - for instance, on $\mathrm{PET} / \mathrm{CT}$ as opposed to ultrasound - remains unclear.

Table 6.3 Per patient overview of SUVmax measurements on PET/CT and hybrid PET/MRI.

\begin{tabular}{|c|c|c|}
\hline Patient & $\begin{array}{l}\mathrm{PET} / \mathrm{CT} \\
\text { SUVmax }\end{array}$ & $\begin{array}{c}\text { Hybrid PET/MRI } \\
\text { SUVmax }\end{array}$ \\
\hline \multicolumn{3}{|l|}{1} \\
\hline Primary tumour & 9.25 & 12.00 \\
\hline Axillary lymph node & 8.70 & 11.03 \\
\hline \multicolumn{3}{|l|}{2} \\
\hline Primary tumour & 11.10 & 11.70 \\
\hline Axillary lymph node & 16.00 & 20.05 \\
\hline \multicolumn{3}{|l|}{3} \\
\hline Primary tumour & 8.80 & 7.60 \\
\hline Axillary lymph node & $7 \cdot 70$ & 4.60 \\
\hline \multicolumn{3}{|l|}{4} \\
\hline Primary tumour & 5.10 & 10.30 \\
\hline Axillary lymph node & 1.95 & 1.70 \\
\hline \multicolumn{3}{|l|}{5} \\
\hline Primary tumour & 19.50 & 17.10 \\
\hline Axillary lymph node & 11.90 & 8.50 \\
\hline \multicolumn{3}{|l|}{6} \\
\hline Primary tumour & 3.40 & 3.90 \\
\hline Axillary lymph node & 6.60 & $5 \cdot 70$ \\
\hline \multicolumn{3}{|l|}{7} \\
\hline Primary tumour & 1.80 & 1.50 \\
\hline Axillary lymph node & $4 \cdot 30$ & 5.40 \\
\hline \multicolumn{3}{|l|}{8} \\
\hline Primary tumour & 3.95 & 4.45 \\
\hline Axillary lymph node & 3.70 & 4.95 \\
\hline \multicolumn{3}{|l|}{9} \\
\hline Primary tumour & 14.05 & 12.05 \\
\hline Axillary lymph node & 7.90 & 7.95 \\
\hline \multicolumn{3}{|l|}{ Mean } \\
\hline Primary tumour & 8.55 & 8.96 \\
\hline Axillary lymph node & 7.65 & 7.76 \\
\hline
\end{tabular}

Abbreviations: PET Positron Emission Tomography, CT Computed Tomography, MRI Magnetic Resonance Imaging, SUVmax maximum standard uptake value. 
In contrast to our study, Grueneisen et al. found no differences of PET/MRI findings compared to PET/CT and MRI for axillary nodal staging in breast cancer patients..$^{20}$ However, in that study, a (standard) breast PET/MRI protocol was used, unlike the state-of-the-art dedicated axillary PET/MRI protocol in our study. In addition, a previous study demonstrated that in approximately $40 \%$ of all patients who underwent standard breast MRI, the field of view of the axillary region was not complete. ${ }^{6}$ In contrast to Grueneisen, a study by Melsaether et al. showed improved diagnostic accuracy of PET/MR imaging versus PET/CT for axillary nodal staging in breast cancer patients, with an improvement in sensitivity from $88 \%$ to $100 \%{ }^{21}$ Nonetheless, their study differentiated only nodenegative from node-positive disease, rather than considering the number of positive nodes. The results of our study can thus be considered to provide added clinical value compared to standard imaging modalities (US, MRI and PET/CT), further improving a patient-tailored approach for axillary nodal staging in clinically node-positive breast cancer.

The accuracy of MRI for axillary nodal staging in our study may differ from that in standard clinical practice. We used dedicated (coronal) axillary MRI instead of standard (cross-sectional) breast MRI that is most commonly used in clinical practice, which might have resulted in higher accuracy than with standard breast MRI. A previous systematic review by Kuijs et al. similarly demonstrated superior results for axillary nodal staging when a dedicated axillary MRI protocol was used. ${ }^{7}$ However, our study revealed that dedicated axillary hybrid PET/MRI is of added value even compared to dedicated axillary $\mathrm{T} 2 \mathrm{~W}$ - and contrast-enhanced MRI, as it still resulted in changes to clinical nodal status in respectively $75 \%$ and $40 \%$ of cases, respectively. In addition, differences in the performance of PET/CT can occur when PET/CT is performed with hanging breast acquisition rather than standard PET/CT. Teixeira et al. demonstrated that PET/CT with hanging breast acquisition improved detection of regional lymph node metastases over standard PET/CT. ${ }^{22}$

SUVmax measurements of primary tumour (8.6 versus 9.0) and the most FDG-avid axillary lymph node (7.7 versus 7.8 ) in our study were comparable between PET/CT and hybrid PET/MRI, despite different reconstruction protocols between both imaging modalities. Pujara et al. also found a strong correlation in SUVmax between PET/CT and PET/MRI in breast cancer patients, and concluded that SUVs from PET/MRI can be used for quantification of 18F-FDG activity. ${ }^{23}$ However, SUVmax comparisons between FDG-avid axillary lymph nodes on PET/CT and PET/MRI were not reported in their study. The results of our study can thus support the earlier findings of Pujara et al. for using SUVmax of FDGavid axillary nodes on PET/MRI for 18F-FDG activity quantification. 
In the future, whole-body PET/MRI, including an integrated dedicated axillary PET/MRI protocol, might replace whole body PET/CT for locoregional and distant staging in breast cancer, since both locoregional and distant staging seem to be at least as accurate as PET/CT. ${ }^{20,24-26} \mathrm{~A}$ secondary benefit would be the reduction in radiation exposure to patients of up to $64 \%$ compared to PET/CT. ${ }^{21}$ The cost-effectiveness of PET/MRI must also be further investigated.

Limitations of the current study include the missing data concerning correlation of dedicated axillary hybrid PET/MR images and final histopathology of axillary lymph nodes; however, nodal metastases were histopathologically confirmed in all patients. Moreover, FDG-avid axillary lymph nodes can be considered malignant, since previous studies have reported high specificity of 95 $100 \%$ for the detection of axillary lymph node metastases in breast cancer. ${ }^{16,27,28}$ However, because of the lack of initial histopathology in these cases, it is possible that some benign lymph nodes according to FDG-activity on PET/MRI still could have been malignant.

Second, there was a timing difference in the performance of PET/MRI in our cohort. Three out of twelve patients underwent only PET/MRI, instead of PET/CT following PET/MRI. This might have led to differences in SUVmax measurements on PET/MRI between the two subgroups. ${ }^{12}$ These three patients were thus excluded from subgroup analysis regarding SUVmax measurements on PET/MRI and PET/CT.

Third, patients underwent unenhanced low-dose whole body PET/CT rather than diagnostic contrast-enhanced PET/CT. The results of PET/CT should be interpreted with this important limitation in mind.

Finally, hybrid PET/MRI is currently available in only a few hospitals worldwide. Therefore, other techniques should also be further explored to approximate the diagnostic performance of PET/MRI, for instance by combining hanging breast PET images with MRI images. ${ }^{29}$

In conclusion, this feasibility study demonstrated that, compared to standard imaging modalities, dedicated axillary 18F-FDG hybrid PET/MRI appears to improve diagnostic performance for axillary nodal staging in clinically nodepositive breast cancer patients. Further studies are needed to investigate the accuracy of this hybrid modality and to compare its performance with that of other dedicated imaging tools, such as hanging breast PET/CT. 


\section{References}

1. Torre LA, Bray F, Siegel RL, Ferlay J, Lortet-Tieulent J, Jemal A. Global cancer statistics, 2012. CA Cancer J Clin. 2015;65:87-108. .

2. Howlader N, Noone AM, Krapcho M, Miller D, Bishop K, Altekruse SF, Kosary CL, Yu M, Ruhl J, Tatalovich Z, Mariotto A, Lewis DR, Chen HS, Feuer EJ, Cronin KA (eds). SEER Cancer Statistics Review, 1975-2013, National Cancer Institute. Bethesda, MD, http://seer.cancer.gov/ csr/1975_2013/, based on November 2015 SEER data submission, posted to the SEER website, April 2016.

3. Senkus E, Kyriakides S, Ohno S, Penault-Llorca F, Poortmans P, Rutgers E, et al. Primary breast cancer: ESMO Clinical Practice Guidelines for diagnosis, treatment and follow-up. Ann Oncol. 2015;26 Suppl 5:v8-30.

4. Kaufmann M, von Minckwitz G, Mamounas EP, Cameron D, Carey LA, Cristofanilli M, et al. Recommendations from an international consensus conference on the current status and future of neoadjuvant systemic therapy in primary breast cancer. Ann Surg Oncol. 2012;19:1508-16.

5. Schipper RJ, van Roozendaal LM, de Vries B, Pijnappel RM, Beets-Tan RG, Lobbes MB, et al. Axillary ultrasound for preoperative nodal staging in breast cancer patients: is it of added value? Breast. 2013;22:1108-13.

6. van Nijnatten TJ, Ploumen EH, Schipper RJ, Goorts B, Andriessen EH, Vanwetswinkel S, et al. Routine use of standard breast MRI compared to axillary ultrasound for differentiating between no, limited and advanced axillary nodal disease in newly diagnosed breast cancer patients. Eur J Radiol. 2016;85:2288-94.

7. Kuijs VJ, Moossdorff M, Schipper RJ, Beets-Tan RG, Heuts EM, Keymeulen KB, et al. The role of MRI in axillary lymph node imaging in breast cancer patients: a systematic review. Insights Imaging. 2015;6:203-15.

8. Koolen BB, Valdes Olmos RA, Elkhuizen PH, Vogel WV, Vrancken Peeters MJ, Rodenhuis S, et al. Locoregional lymph node involvement on 18F-FDG PET/CT in breast cancer patients scheduled for neoadjuvant chemotherapy. Breast Cancer Res Treat. 2012;135:231-40.

9. Huellner MW, Appenzeller P, Kuhn FP, Husmann L, Pietsch CM, Burger IA, et al. Whole-body nonenhanced PET/MR versus PET/CT in the staging and restaging of cancers: preliminary observations. Radiology. 2014;273:859-69.

10. NABON (2012) National breast cancer guideline, Oncoline. www.oncoline.nl/breastcancer Accessed 17 May 2017.

11. Neal CH, Daly CP, Nees AV, Helvie MA. Can preoperative axillary US help exclude $\mathrm{N}_{2}$ and $\mathrm{N}_{3}$ metastatic breast cancer? Radiology. 2010;257:335-41.

12. Boellaard R, Delgado-Bolton R, Oyen WJ, Giammarile F, Tatsch K, Eschner W, et al. FDG PET/CT: EANM procedure guidelines for tumour imaging: version 2.o. Eur J Nucl Med Mol Imaging. 2015;42:328-54.

13. Baltzer PA, Dietzel M, Burmeister HP, Zoubi R, Gajda M, Camara O, et al. Application of MR mammography beyond local staging: is there a potential to accurately assess axillary lymph nodes? evaluation of an extended protocol in an initial prospective study. AJR Am J Roentgenol. 2011;196:W641-7.

14. Farshchian N, Tamari S, Farshchian N, Madani H, Rezaie M, Mohammadi-Motlagh HR. Diagnostic value of chemical shift artifact in distinguishing benign lymphadenopathy. Eur J Radiol. 2011;80:594-7.

15. Lambregts DM, Heijnen LA, Maas M, Rutten IJ, Martens MH, Backes WH, et al. Gadofosvesetenhanced MRI for the assessment of rectal cancer lymph nodes: predictive criteria. Abdom Imaging. 2013;38:720-7.

16. Aukema TS, Straver ME, Peeters MJ, Russell NS, Gilhuijs KG, Vogel WV, et al. Detection of extraaxillary lymph node involvement with FDG PET/CT in patients with stage II-III breast cancer. Eur J Cancer. 2010;46:3205-10. 
17. Gradishar WJ, Anderson BO, Balassanian R, Blair SL, Burstein HJ, Cyr A, et al. Invasive Breast Cancer Version 1.2016, NCCN Clinical Practice Guidelines in Oncology. J Natl Compr Canc Netw. 2016;14:324-54.

18. Giuliano AE, Ballman K, McCall L, Beitsch P, Whitworth PW, Blumencranz P, et al. Locoregional Recurrence After Sentinel Lymph Node Dissection With or Without Axillary Dissection in Patients With Sentinel Lymph Node Metastases: Long-term Follow-up From the American College of Surgeons Oncology Group (Alliance) ACOSOG Zoo11 Randomized Trial. Ann Surg. 2016;264:413-20.

19. Teixeira SC, Koolen BB, Elkhuizen PH, Vrancken Peeters MT, Stokkel MP, Rodenhuis S, et al. PET/CT with $18 \mathrm{~F}$-FDG predicts short-term outcome in stage II/III breast cancer patients upstaged to N2/3 nodal disease. Eur J Surg Oncol. 2017;43:625-35.

20. Grueneisen J, Nagarajah J, Buchbender C, Hoffmann O, Schaarschmidt BM, Poeppel T, et al. Positron Emission Tomography/Magnetic Resonance Imaging for Local Tumor Staging in Patients With Primary Breast Cancer: A Comparison With Positron Emission Tomography/Computed Tomography and Magnetic Resonance Imaging. Invest Radiol. 2015;50: 505-13.

21. Melsaether AN, Raad RA, Pujara AC, Ponzo FD, Pysarenko KM, Jhaveri K, et al. Comparison of Whole-Body (18)F FDG PET/MR Imaging and Whole-Body (18)F FDG PET/CT in Terms of Lesion Detection and Radiation Dose in Patients with Breast Cancer. Radiology. 2016;281:193-202.

22. Teixeira SC, Koolen BB, Vogel WV, Wesseling J, Stokkel MP, Vrancken Peeters MJ, et al. Additional Prone 18F-FDG PET/CT Acquisition to Improve the Visualization of the Primary Tumor and Regional Lymph Node Metastases in Stage II/III Breast Cancer. Clin Nucl Med. 2016;41:e181-6.

23. Pujara AC, Raad RA, Ponzo F, Wassong C, Babb JS, Moy L, et al. Standardized Uptake Values from PET/MRI in Metastatic Breast Cancer: An Organ-based Comparison With PET/CT. Breast J. 2016;22:264-73.

24. Pace L, Nicolai E, Luongo A, Aiello M, Catalano OA, Soricelli A, et al. Comparison of whole-body $\mathrm{PET} / \mathrm{CT}$ and PET/MRI in breast cancer patients: lesion detection and quantitation of 18Fdeoxyglucose uptake in lesions and in normal organ tissues. Eur J Radiol. 2014;83:289-96.

25. Sawicki LM, Grueneisen J, Schaarschmidt BM, Buchbender C, Nagarajah J, Umutlu L, et al. Evaluation of (18)F-FDG PET/MRI, (18)F-FDG PET/CT, MRI, and CT in whole-body staging of recurrent breast cancer. Eur J Radiol. 2016;85:459-65.

26. Segaert I, Mottaghy F, Ceyssens S, De Wever W, Stroobants S, Van Ongeval C, et al. Additional value of PET-CT in staging of clinical stage IIB and III breast cancer. Breast J. 2010;16:617-24.

27. Fuster D, Duch J, Paredes P, Velasco M, Munoz M, Santamaria G, et al. Preoperative staging of large primary breast cancer with $[18 \mathrm{~F}]$ fluorodeoxyglucose positron emission tomography/ computed tomography compared with conventional imaging procedures. J Clin Oncol. 2008;26:4746-51.

28. Veronesi U, De Cicco C, Galimberti VE, Fernandez JR, Rotmensz N, Viale G, et al. A comparative study on the value of FDG-PET and sentinel node biopsy to identify occult axillary metastases. Ann Oncol. 2007;18:473-8.

29. Teixeira SC, Rebolleda JF, Koolen BB, Wesseling J, Jurado RS, Stokkel MP, et al. Evaluation of a Hanging-Breast PET System for Primary Tumor Visualization in Patients With Stage I-III Breast Cancer: Comparison With Standard PET/CT. AJR Am J Roentgenol. 2016;206:1307-14. 


\section{Appendix 6.1}

Complete overview of all axillary lymph nodes evaluated on (PET)/MRI and PET/CT exams.

\begin{tabular}{|c|c|c|c|c|c|c|c|c|}
\hline & \multicolumn{2}{|c|}{ T2W-MRI } & \multicolumn{2}{|c|}{ CE-TiW-MRI } & \multicolumn{2}{|c|}{ Hybrid PET/MRI } & \multicolumn{2}{|c|}{$\mathrm{PET} / \mathrm{CT}$} \\
\hline & $\begin{array}{c}\text { Benign } \\
\text { (score } 0-2 \text { ) } \\
\end{array}$ & $\begin{array}{l}\text { Suspicious } \\
(\text { score 3-4) } \\
\end{array}$ & $\begin{array}{c}\text { Benign } \\
\text { (score o-1) } \\
\end{array}$ & $\begin{array}{l}\text { Suspicious } \\
(\text { score 2-3) } \\
\end{array}$ & $\begin{array}{c}\text { Benign } \\
(\text { score 0-1) } \\
\end{array}$ & $\begin{array}{l}\text { Suspicious } \\
\text { (score 2-3) }\end{array}$ & $\begin{array}{c}\text { Benign } \\
(\text { score o-1) } \\
\end{array}$ & $\begin{array}{l}\text { Suspicious } \\
(\text { score 2-3) } \\
\end{array}$ \\
\hline \multicolumn{9}{|c|}{ 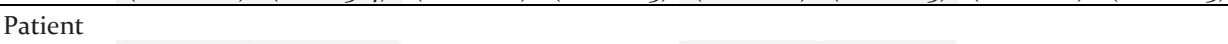 } \\
\hline 1 & 2 & 10 & 2 & 10 & 4 & 8 & 1 & 5 \\
\hline 2 & 11 & o & 10 & 1 & 9 & 2 & - & - \\
\hline 3 & 15 & 5 & 13 & 7 & 19 & 1 & - & - \\
\hline 4 & 2 & 10 & 3 & 9 & 4 & 8 & o & 5 \\
\hline 5 & 4 & 3 & - & - & o & 7 & 2 & 9 \\
\hline 6 & 3 & 25 & 17 & 11 & 26 & 2 & 5 & 2 \\
\hline 7 & 6 & 4 & - & - & 7 & 3 & o & 4 \\
\hline 8 & 9 & 6 & 8 & 7 & 12 & 3 & o & 3 \\
\hline 9 & 4 & 10 & 1 & 13 & 4 & 10 & o & 7 \\
\hline 10 & 9 & 2 & 9 & 2 & 10 & 1 & o & 1 \\
\hline $11^{*}$ & 5 & 3 & 5 & 5 & 6 & 4 & - & - \\
\hline 12 & 4 & 2 & 3 & 3 & 5 & 1 & $\mathrm{o}$ & 1 \\
\hline
\end{tabular}

* This concerned the patient with mixed lobular carcinoma with carcinoma of no special type.

Abbreviations: $T_{2} W T_{2}$-weighted, $M R I$ magnetic resonance imaging, $C E-T_{1} W$ contrast-enhanced T1-weighted, PET Positron Emission Tomography, CT Computed Tomography. 


\section{Part III}

Classification of lymph node staging 



\section{Chapter}

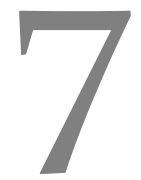

TNM classification and the need for revision of pN3a breast cancer

TJA van Nijnatten, M Moossdorff, L de Munck, B Goorts, MLG Vane, KBMI Keymeulen, RGH Beets-Tan, MBI Lobbes, ML Smidt

Eur J Cancer 2017;79:23-30 


\section{Abstract}

\section{Background}

According to the seventh edition of tumour-node-metastasis (TNM) classification, pN3a status in breast cancer patients consists of presence of an infraclavicular lymph node metastasis (LNM) and/or presence of $\geq 10$ axillary LNMs. The aim of this study was to determine whether prognosis of $\mathrm{pN}_{3}$ a based on at least an infraclavicular LNM differs from $\geq 10$ axillary LNMs.

\section{Methods}

Data were obtained from the Netherlands Cancer Registry. All patients were diagnosed between 2005 and 2008 with primary invasive epithelial breast cancer and $\mathrm{pN}_{2 \mathrm{a}}$ or $\mathrm{pN}_{3} \mathrm{a}$ status as pathologic result. Patients with $\mathrm{pN}_{3}$ a were subdivided in $\mathrm{pN}_{3}$ a based on at least an infraclavicular LNM or $\geq 10$ axillary LNMs. Diseasefree survival (DFS) included any local, regional or contralateral recurrence, distant metastasis or death within 5 years. Kaplan-Meier curves provided information on 5-year DFS and 8-year overall survival (OS). In addition, Cox proportional hazards model was used to measure the effect of relevant clinicopathological variables on DFS and OS.

\section{Results}

A total of 3400 patients with pN2a and 1788 patients with pN3a were included. In 83 patients, pN3a was based on at least an infraclavicular LNM (4.6\%) and in 1705 patients because of $\geq 10$ axillary LNMs (95.4\%). After multivariable analyses, DFS and OS were inferior in patients with $\mathrm{pN}_{3}$ a based on $\geq 10$ axillary LNMs compared to infraclavicular LNM (DFS $48.8 \%$ versus $63.8 \%$, hazard ratio [HR] 1.59, $P=0.036$; OS $46.6 \%$ versus $63.9 \%$, HR 1.46, $P=0.042)$. Furthermore, pN2a and pN3a based on infraclavicular LNM had comparable DFS and OS.

\section{Conclusion}

$\mathrm{PN}_{3}$ a status based on an at least an infraclavicular LNM is rare, yet its prognosis is superior to $\geq 10$ axillary LNMs. Reclassification of infraclavicular LNM in the next TNM should therefore be considered into pN2a. 


\section{Introduction}

In 1958, the first edition of the tumour-node-metastasis (TNM) classification of malignant tumours of the breast was published by the Union for International Cancer Control (UICC) in order to achieve worldwide consensus for the classification of, eventually, each solid tumour type. ${ }^{1}$ Subsequently, this classification system was revised each decade to implement new insights. For instance, the introduction of neoadjuvant systemic therapy, sentinel lymph node biopsy, immunohistochemical staining and the method of pathologic nodal staging. ${ }^{2}$

Regarding pathologic nodal staging, axillary lymph node metastases (LNMs) were divided into three categories in the fifth edition of the TNM classification: pNo (o axillary LNMs), pN1 (movable axillary LNMs) and pN2 (fixed axillary LNMs). ${ }^{3}$ After revision in sixth edition, the number of axillary LNMs was incorporated as key element in the classification, as impaired prognosis was demonstrated in the presence of an increasing number of axillary LNMs. ${ }^{4}$ This resulted in four categories: pNo (o axillary LNMs), pNia (1-3 axillary LNMs), pN2a (4-9 axillary LNMs) and pN3a ( $\geq 10$ axillary LNMs). ${ }^{5}$ Furthermore, a study by Newman et al. observed a worse disease-free (DFS) and overall survival (OS) in patients with infraclavicular (level III) and axillary LNMs compared to patients with axillary LNMs only (DFS $50 \%$ versus $68 \%$; OS $58 \%$ versus $83 \%$, respectively). ${ }^{6}$ As a consequence, the UICC decided to redefine infraclavicular LNM as pN3a in the sixth edition; in contrast to earlier, when an infraclavicular LNM was considered equivalent to other axillary LNMs in the fifth edition. Currently $\mathrm{pN}_{3} \mathrm{a}$ nodal status consists of patients with $\geq 10$ axillary LNMs and of patients with infraclavicular LNM. ${ }^{7}$

The combination of both groups within pN3a suggests that their prognosis is similar. ${ }^{8,9}$ However, no study thus far analysed this assumption. Therefore, the purpose of this study is to determine whether the prognosis of $\mathrm{pN}_{3}$ a breast cancer patients based on at least an infraclavicular LNM is different compared to patients with $\geq 10$ axillary LNMs and to patients with 4-9 axillary LNMs.

\section{Materials and methods}

\section{Data collection}

Data were obtained from the Netherlands Cancer Registry (NCR), managed by the Netherlands Comprehensive Cancer Organisation (IKNL). The NCR collects data of all patients diagnosed with any type of cancer in the Netherlands, after a notification of the PALGA ('Nationwide network and registry of histo- and 
cytopathology in the Netherlands') system. Afterwards, trained data collection registrars from the NCR extracted data from patients' records concerning patient characteristics, treatment and follow-up.

In this study, all patients diagnosed between 2005 and 2008 with primary invasive epithelial breast cancer and $\mathrm{pN}_{2} \mathrm{a}$ or $\mathrm{pN}_{3}$ a statuses as the final pathologic result were included. Exclusion criteria were synchronous breast cancer, distant metastases at time of diagnosis (or within 91 days) or an unknown number of LNMs. Patients without surgery were also excluded. Data were collected on age, tumour type, receptor status, surgical procedures, systemic therapy, radiation therapy and pathological results, including pathologic TNM classification and the number of LNMs. For a period of 5 years after diagnosis, the first breast cancer event was registered, which consisted of any local, regional or contralateral recurrence or distant metastasis.

Patients with $\mathrm{pN}_{3}$ a were divided into two subgroups according to the number of LNMs, to simulate $\mathrm{pN}_{3}$ a based on infraclavicular or $\geq 10$ axillary LNMs. Patients with nine or less positive lymph nodes required at least one infraclavicular LNM to be considered $\mathrm{pN}_{3} \mathrm{a}$, while patients with $\geq 10$ positive lymph nodes required at least 10 axillary LNMs (with or without infraclavicular LNMs).

\section{Treatment}

According to the national guideline of 2005, regional treatment depended on nodal status: sentinel lymph node biopsy (SLNB) in case of clinically node negative status, based on physical examination (axillary ultrasound was common but not mandatory at that time), or axillary lymph node dissection (ALND) in case of clinically node positive status, contraindication for SLNB or positive SLNB. ${ }^{10}$

Adjuvant irradiation of regional nodal fields was applied in case of four or more axillary LNMs or involvement of top axillary LNM. Recommended dose was 45-50 Gy in 5 weeks.

Systemic therapy was generally recommended for all patients with LNM. Chemotherapy was advised in all premenopausal women and in women $<69$ years old with estrogen (ER) and progesterone (PR) tumours. In postmenopausal women, aged 50-59 years with ER+ PR+, chemotherapy was considered in physically fit patients and in women aged 6o-69 years only if four or more nodes were involved. Chemotherapy regimen consisted of five courses 5 Fluorouracil, Epirubicin, Cyclophosphamide or six courses of Taxotere, Adriamycin and Cyclophosphamide. In case of Her2Neu receptor (HER 2) amplification, targeted therapy (Trastuzumab) was given in addition to chemotherapy. Endocrine therapy was recommended for all ER+ and/or PR+ tumours. 


\section{Statistics}

Statistical analyses were performed by using Statistical Package for the Social Sciences software (Version 22, IBM, Armonk, New York, USA). General characteristics between both subgroups were compared using chi-squared test for categorical data and Mann-Whitney U-test for continuous data.

For DFS, an event was defined as any local, regional or contralateral recurrence, distant metastasis or mortality within 5 years after the primary diagnosis. Events occurring o-91 days after diagnosis were considered synchronous to the original tumour and not counted as recurrences. Date of death or date of emigration were derived from the Municipal Personal Records Database and completed until 31st December 2014. Patients were censored at the date of their first event, date of last follow-up, date of death or date of emigration, whatever came first.

DFS and OS for the pN3a subgroups, respectively, based on an infraclavicular LNM and $\geq 10$ axillary LNMs, were calculated with Kaplan-Meier curves and compared with the log-rank test. ${ }^{11} P$-Values (two-sided) $<0.05$ were considered statistically significant. Relevant clinicopathological variables associated with DFS and OS were examined using univariable and, where applicable, multivariable Cox proportional hazards regression, with hazard ratio (HR) and corresponding 95\% confidence intervals. The number of variables used for multivariable Cox proportional hazards regression depends on the number of outcome events per predictor variable, which requires at least five events per variable. ${ }^{12}$

Finally, DFS and OS of patients with pN3a based on infraclavicular LNM were compared to patients with pN2a (i.e. 4-9 axillary LNMs), by calculating KaplanMeier curves and comparing with the log-rank test. In addition, univariable and multivariable Cox proportional hazards regression evaluated association of relevant clinicopathological variables associated with DFS and OS.

\section{Results}

A total of 51,239 patients were diagnosed with primary invasive epithelial breast cancer between 2005 and 2008 in the Netherlands, of whom 3442 patients had pN2a (6.6\%) and 1799 patients (3.5\%) had pN3a status (Figure 7.1). Eventually, 83 patients were classified as $\mathrm{pN}_{3}$ a based on infraclavicular LNM (4.6\%) and 1705 patients based on $\geq 10$ axillary LNMs (95.4\%). Compared to patients with $\geq 10$ axillary LNMs, patients with at least an infraclavicular LNM were younger (55 versus 59 years, $P=0.010$ ), less often had $\mathrm{pT}_{3}-4$ tumours ( $15 \%$ versus $24 \%$, $P=0.049$ ) with a smaller mean tumour size (31 versus $36 \mathrm{~mm}, P=0.032)$ and, obviously, had fewer positive lymph nodes (mean 6 versus 15, $P<0.001$ ). A more detailed overview of the general characteristics is provided in Table 7.1. 


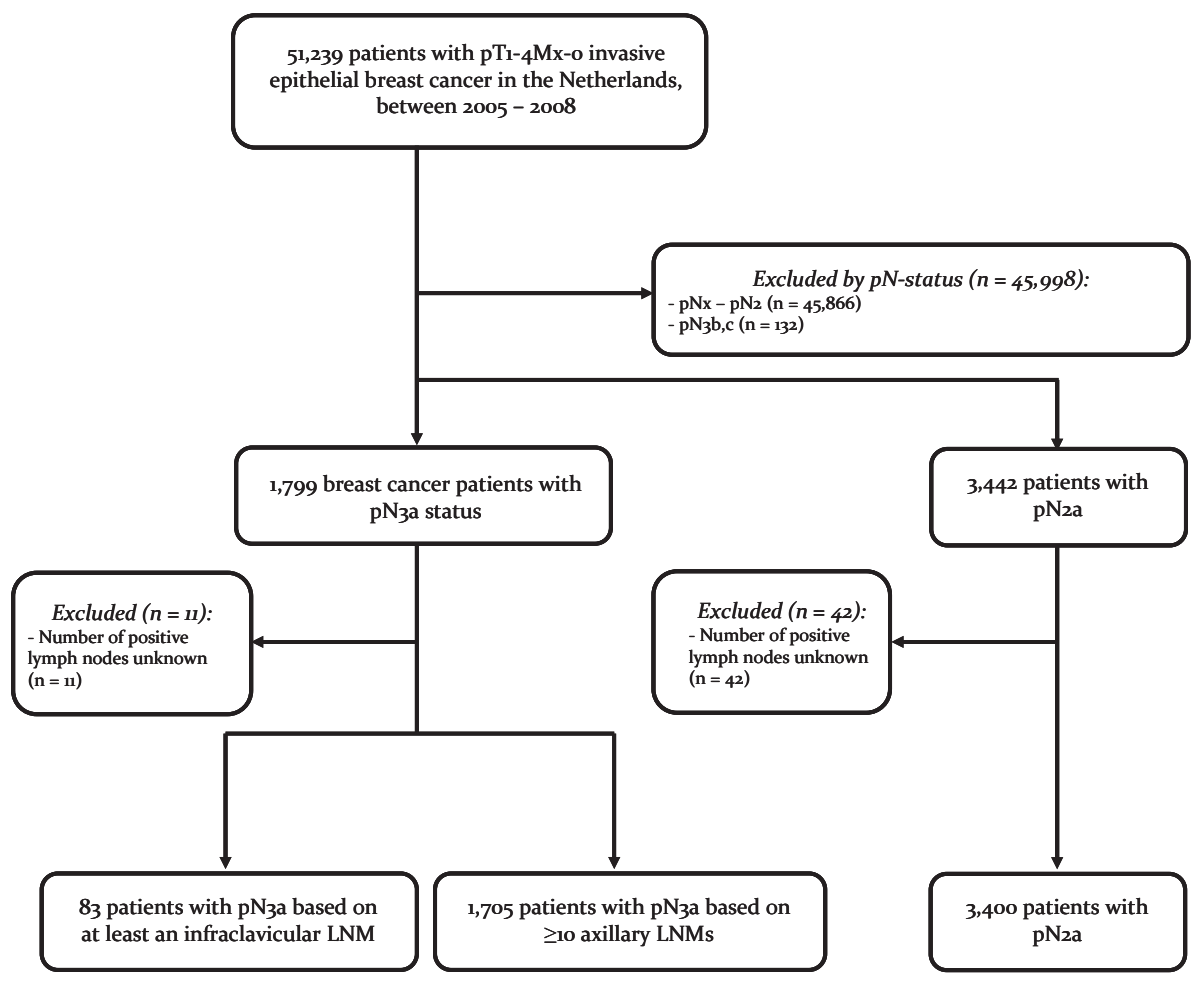

Figure 7.1 Flowchart of included patients. Abbreviations: $p N$ pathologic nodal status, LNM lymph node metastases.

Table 7.1 General patient and tumour characteristics.

\begin{tabular}{lccc}
\hline & $\begin{array}{c}\text { pN3a Infraclavicular } \\
\text { LNM } \\
(\mathrm{n}=83)\end{array}$ & $\begin{array}{c}\text { pN3a } \geq 10 \text { axillary } \\
\text { LNMs } \\
(\mathrm{n}=1705)\end{array}$ & $P$-value \\
\hline Mean age (years) (range) & $55.3(30-84)$ & $59.1(26-97)$ & 0.010 \\
Pathologic T-stage (\%) & & & \\
To-2 & $69(83.1)$ & $1252(73.4)$ & 0.049 \\
T3-4 & $12(14.5)$ & $406(23.8)$ & 0.049 \\
Unknown & $2(2.4)$ & $47(2.8)$ & 0.850 \\
Mean tumour size (mm) (range) & $31.4(6-114)$ & $35.6(0-250)$ & 0.032 \\
Tumour grade (\%) & & & \\
$1-2$ & $39(47.0)$ & $785(46.0)$ & 0.284 \\
3 & $33(39.7)$ & $220(12.9)$ & 0.926 \\
Unknown & $11(13.3)$ & $15.2(10-53)$ & $<0.001$ \\
Mean number of positive lymph nodes (range) & $5.7(1-9)$ & & 0.405 \\
ER (\%) & & $1244(73.0)$ & 0.472 \\
Positive & $64(77.1)$ & $451(26.4)$ & 1.000 \\
Negative & $19(22.9)$ & $10(0.6)$ & 0 \\
Unknown & &
\end{tabular}


Table 7.1 (continued)

\begin{tabular}{|c|c|c|c|}
\hline & $\begin{array}{c}\text { pN3a Infraclavicular } \\
\text { LNM } \\
(\mathrm{n}=83) \\
\end{array}$ & $\begin{array}{c}\text { pN3a } \geq 10 \text { axillary } \\
\text { LNMs } \\
(\mathrm{n}=1705) \\
\end{array}$ & $P$-value \\
\hline \multicolumn{4}{|l|}{ PR (\%) } \\
\hline Positive & $48(57.8)$ & $896(52.6)$ & 0.347 \\
\hline Negative & $34(41.0)$ & $723(42.4)$ & 0.795 \\
\hline Unknown & $1(1.2)$ & $86(5.0)$ & 0.183 \\
\hline \multicolumn{4}{|l|}{ Her2 (\%) } \\
\hline Positive & $12(14.5)$ & $346(20.3)$ & 0.195 \\
\hline Negative & $65(78.3)$ & $1247(73.1)$ & 0.298 \\
\hline Equivocal & $3(3.6)$ & $59(3.5)$ & 0.763 \\
\hline Unknown & $3(3.6)$ & $53(3.1)$ & 0.743 \\
\hline \multicolumn{4}{|l|}{ Tumour type (\%) } \\
\hline Invasive carcinoma NST & $5^{8}(69.9)$ & $1151(67.5)$ & 0.652 \\
\hline Lobular & $15(18.1)$ & $344(20.2)$ & 0.640 \\
\hline Mixed ductal and lobular & $5(6.0)$ & $92(5.4)$ & 0.805 \\
\hline Other & $5(6.0)$ & $118(6.9)$ & 0.753 \\
\hline \multicolumn{4}{|l|}{ Subtype (\%) } \\
\hline $\mathrm{ER}+\mathrm{PR}+$, Her2- & $41(49 \cdot 4)$ & $747(43.8)$ & 0.224 \\
\hline ER+PR-, Her2- & $13(15 \cdot 7)$ & $241(14.1)$ & 0.697 \\
\hline ER+Her2+ & $5(6.0)$ & $168(9.9)$ & 0.249 \\
\hline ER-Her2+ & $7(8.4)$ & $174(10.2)$ & 0.601 \\
\hline Triple negative & $12(14.5)$ & $238(14.0)$ & 0.898 \\
\hline Unknown & $4(4.8)$ & $127(7.4)$ & 0.369 \\
\hline \multicolumn{4}{|l|}{ Breast surgery (\%) } \\
\hline Breast conserving therapy & $22(26.5)$ & $339(19 \cdot 9)$ & 0.142 \\
\hline Mastectomy & $61(73.5)$ & $1363(79.9)$ & 0.159 \\
\hline Unknown & o & $3(0.2)$ & 1.000 \\
\hline \multicolumn{4}{|l|}{ Axillary surgery (\%) } \\
\hline SLNB & $4(4.8)$ & $2(0.1)$ & $<0.001$ \\
\hline SLNB followed by ALND & $26(31.3)$ & $316(18.6)$ & 0.004 \\
\hline ALND & $53(63.9)$ & $1378(80.8)$ & $<0.001$ \\
\hline Unknown & o & $9(0.5)$ & 1.000 \\
\hline \multicolumn{4}{|l|}{ Radiation therapy (\%) } \\
\hline Yes & $76(91.6)$ & $1528(89.6)$ & 0.569 \\
\hline \multicolumn{4}{|l|}{ Chemotherapy (\%) } \\
\hline Yes & $67(80.7)$ & $1267(74 \cdot 3)$ & 0.190 \\
\hline \multicolumn{4}{|c|}{ Endocrine therapy to ER+ subtype (\%) } \\
\hline \multicolumn{4}{|c|}{ Trastuzumab to Herz+ subtype (\%) } \\
\hline Yes & $8(66.7)$ & $254(73.4)$ & 0.604 \\
\hline
\end{tabular}

Abbreviations: ER estrogen, $P R$ progesteron, Her2 human epidermal growth factor receptor 2, SLNB sentinel lymph node biopsy, $A L N D$ axillary lymph node dissection.

\section{Disease-free survival}

Five year follow-up was available for 1293 patients $(72.3 \%, \mathrm{n}=58$ versus $\mathrm{n}=1235$, for patients with at least an infraclavicular versus $\geq 10$ axillary LNMs). Within 5 years after diagnosis, $43.6 \%$ experienced a first locoregional or contralateral recurrence 
or distance metastasis and $6.9 \%$ deceased. Thus $50.5 \%$ of the patients experienced an event, resulting in a DFS of $49.5 \%$. In subgroup analyses, DFS was $63.8 \%$ in patients with at least an infraclavicular LNM and $48.8 \%$ of patients with $\geq 10$ axillary LNMs $(P=0.018)$ (Figure 7.2a).

In multivariable Cox regression analyses, the effect of having $\geq 10$ axillary LNMs on DFS was significant (HR 1.59, $P=0.036)$ (Table 7.2). Receiving chemotherapy (HR 0.51, $P<0.001$ ) and radiation therapy (HR 0.59, $P<0.001$ ) were identified as significant predictors for increased DFS, whereas triple negative subtype (HR 2.57, $P<$ o.oo1) was identified as significant predictor for decreased DFS.

A

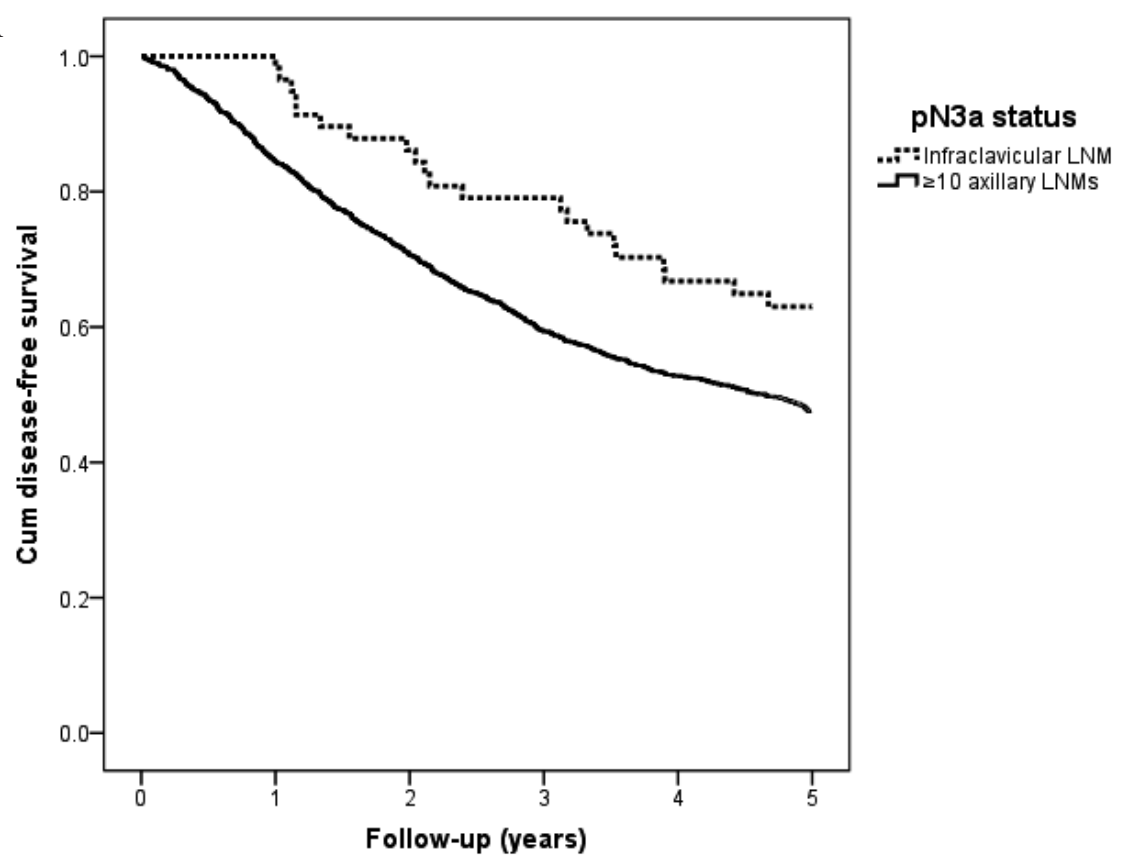

\begin{tabular}{lcccccc}
\hline $\begin{array}{l}\text { Infraclavicular } \\
\text { LNM }\end{array}$ & 58 & 57 & 49 & 45 & 37 & 23 \\
\hline $\begin{array}{l}\geq 10 \text { axillary } \\
\text { LNMs }\end{array}$ & 1235 & 1040 & 865 & 721 & 625 & 356 \\
\hline
\end{tabular}


B

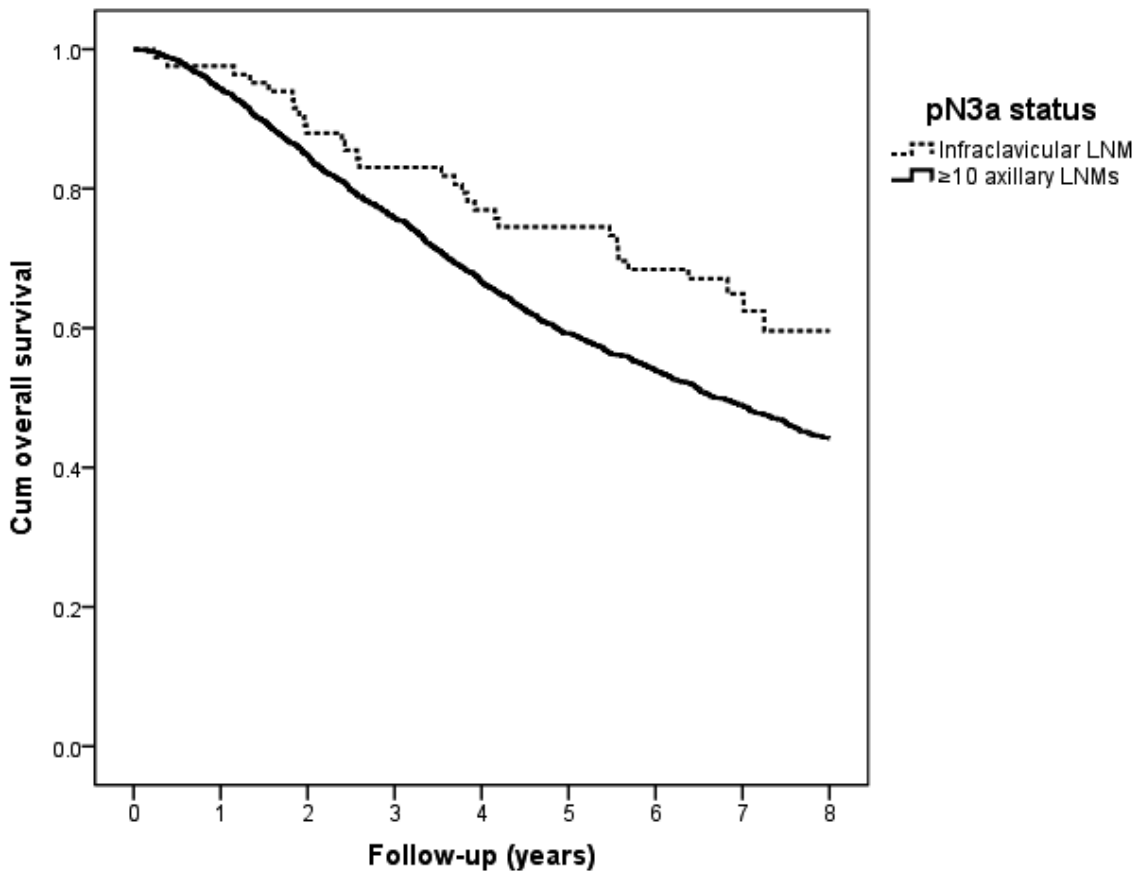

\begin{tabular}{lccccccccc}
\hline $\begin{array}{l}\text { Infraclavicular } \\
\text { LNM }\end{array}$ & 83 & 81 & 73 & 68 & 63 & 61 & 56 & 26 & 12 \\
\hline $\begin{array}{l}\geq 10 \text { axillary } \\
\text { LNMs }\end{array}$ & 1705 & 1607 & 1444 & 1288 & 1130 & 1004 & 912 & 627 & 373 \\
\hline
\end{tabular}

Figure 7.2 (A) and (B) Kaplan-Meier curves for disease-free survival after 5 years of follow up and overall survival after 8 years, including the number of patients at risk. LNM, lymph node metastases.

\section{Overall survival}

After 8 years of follow-up, $47.4 \%$ of all patients were alive. This concerned $63.9 \%$ of patients with at least an infraclavicular LNM and $46.6 \%$ with $\geq 10$ axillary LNMs $(P=0.009)$ (Figure 7.2b).

In multivariable Cox regression analyses, the effect of having $\geq 10$ axillary LNMs on OS was statistically significant (HR 1.46, $P=0.042$ ) (Table 7.3). Significant predictors for decreased OS were the presence of $\mathrm{pT}_{3}-4$ tumours (HR 1.60, $P<0.001)$ and triple negative subtype (HR 1.79, $P<0.001)$. Receiving chemotherapy (HR 0.42, $P<0.001$ ), endocrine therapy (HR o.6o, $P<0.001$ ) and radiation therapy (HR 0.53, $P<$ o.oo1) were identified as significant predictors for increased OS. 


\section{Comparison of infraclavicular LNM to pN2a nodal status}

In the subgroup of $\mathrm{pN} 2 \mathrm{a}, 5$-year follow-up was available for 2483 patients $(73.0 \%)$ with a DFS of $67.3 \%$. Compared to patients with pN3a based on infraclavicular LNM (DFS 63.8\%) this was not statistically significant $(P=0.661$ ) (Appendix 7.1a). In multivariable Cox regression analyses, the effect of having $\mathrm{pN}_{3}$ a based on infraclavicular LNM on DFS remained comparable to pN2a (HR 1.17, $P=0.491$ ) (Appendix 7.2a).

After 8 years of follow-up, $65.5 \%$ of pNza patients were alive. Again, which was not different compared to OS of patients with $\mathrm{pN}_{3}$ a based on infraclavicular LNM (OS 63.9\%) $(P=0.500)$ (Appendix 7.1b). In multivariable Cox regression analyses, the effect of having pN3a based on infraclavicular LNM on OS remained comparable to pN2a (HR 1.25, $P=0.233$ ) Appendix 7.2b).

Table 7.2 Uni- and multivariable analyses of predictors for disease-free survival.

\begin{tabular}{lcccc}
\hline & \multicolumn{2}{c}{ Univariable analysis } & \multicolumn{2}{c}{ Multivariable analysis } \\
\cline { 2 - 5 } & \multicolumn{1}{c}{ HR $(95 \% \mathrm{CI})$} & $P$-value & HR $(95 \% \mathrm{CI})$ & $P$-value \\
\hline Infraclavicular LNM & Reference & & Reference & 0.036 \\
$\geq 1$ 0 axillary LNMs & $1.68(1.09-2.59)$ & 0.020 & $1.59(1.03-2.46)$ & - \\
Age (per year increment) & $1.02(1.02-1.03)$ & $<0.001$ & - & - \\
pT-stage T3-4 vs. To-2 & $1.65(1.39-1.96)$ & $<0.001$ & - & - \\
Tumour grade 3 vs. 1-2 & $1.45(1.24-1.69)$ & $<0.001$ & - & $<0.001$ \\
Triple negative subtype Yes vs. No & $2.43(2.01-2.94)$ & $<0.001$ & $2.57(2.13-3.11)$ & - \\
\hline Chemotherapy Yes vs. No & $0.49(0.42-0.58)$ & $<0.001$ & $0.51(0.43-0.60)$ & - \\
Trastuzumab Yes vs. No & $0.74(0.59-0.92)$ & 0.007 & - & - \\
\hline Endocrine therapy Yes vs. No & $0.50(0.43-0.59)$ & $<0.001$ & $<0.001$ \\
Radiation therapy Yes vs. No & $0.48(0.37-0.60)$ & $<0.001$ & $0.59(0.46-0.75)$ & $<$ \\
\hline
\end{tabular}

Abbreviations: LNM lymph node metastases, $p T$-stage pathologic tumour stage.

Table 7.3 Uni- and multivariable analyses of predictors for overall survival.

\begin{tabular}{lcccc}
\hline & \multicolumn{2}{c}{ Univariable analysis } & \multicolumn{2}{c}{ Multivariable analysis } \\
\cline { 2 - 5 } & HR $(95 \% \mathrm{CI})$ & $P$-value & HR $(95 \% \mathrm{CI})$ & $P$-value \\
\hline Infraclavicular LNM & Reference & & Reference & \\
$\geq 10$ axillary LNMs & $1.61(1.12-2.32)$ & 0.010 & $1.46(1.01-2.10)$ & 0.042 \\
Age (per year increment) & $1.03(1.03-1.04)$ & $<0.001$ & - & - \\
pT-stage T3-4 vs To-2 & $1.56(1.35-1.79)$ & $<0.001$ & $1.60(1.39-1.85)$ & $<0.001$ \\
\hline Tumour grade 3 vs 1-2 & $1.43(1.26-1.63)$ & $<0.001$ & - & - \\
Triple negative subtype Yes vs No & $2.38(2.03-2.80)$ & $<0.001$ & $1.79(1.47-2.19)$ & $<0.001$ \\
\hline Chemotherapy Yes vs No & $0.42(0.36-0.48)$ & $<0.001$ & $0.42(0.36-0.48)$ & $<0.001$ \\
Trastuzumab Yes vs No & $0.64(0.53-0.78)$ & $<0.001$ & - & - \\
\hline Endocrine therapy Yes vs No & $0.51(0.45-0.58)$ & $<0.001$ & $0.60(0.51-0.71)$ & $<0.001$ \\
Radiation therapy Yes vs No & $0.38(0.32-0.45)$ & $<0.001$ & $0.53(0.44-0.64)$ & $<0.001$ \\
\hline
\end{tabular}

Abbreviations: LNM lymph node metastases, $p T$-stage pathologic tumour stage. 


\section{Discussion}

According to the sixth and seventh edition of the TNM classification for breast cancer, pathologic nodal status is defined using the number and location of LNMs. A pN3a status in breast cancer consists either of at least an infraclavicular (level III) or $\geq 10$ axillary LNMs. ${ }^{7}$ Inclusion of both groups in the same category of TNM suggests a similar prognosis. ${ }^{8,9,13}$ However, our study demonstrated superior DFS and OS in patients with pN3a based on at least an infraclavicular LNM compared to $\geq 10$ axillary LNMs. Furthermore, DFS and OS of patients with pN3a based on at least an infraclavicular LNM compared to patients with pN2a were comparable according to our study.

The decision to redefine infraclavicular LNM as pN3a breast cancer was solely based on the results of a study of Newman et al.. ${ }^{2,6}$ In this study, incidence and prognosis of infraclavicular LNM among patients with axillary LNMs was investigated, which showed worse DFS and OS in case of suspicious adenopathy. ${ }^{6}$ Study limitations were the absence of pathological confirmation of the suspicious nodes, as well as potentially confounding factors like presence of supraclavicular LNM in some patients. ${ }^{14}$ In our study, pN3a was defined according to final pathological report, resulting in pathologically confirmed infraclavicular LNM in all 83 patients and therefore representing a more valid patient population.

Classification of infraclavicular LNM as $\mathrm{pN}_{3}$ a disregards the number of nodal metastases and the size of the largest metastasis. A single micrometastasis in an infraclavicular lymph node would represent $\mathrm{pN}_{3}$ a status, whereas $\mathrm{pN} 3 \mathrm{a}$ without infraclavicular involvement would require $\geq 10$ LNMs with at least one macrometastasis. ${ }^{7}$ Disregarding size of the nodal metastases by only taking infraclavicular location into account can explain part of the difference in DFS and OS between pN3a based on at least an infraclavicular LNM and $\geq 10$ axillary LNMs.

DFS after 5 years in patients with $\geq 10$ axillary LNMs in our study cohort is comparable to previous results of Koca et al., in which 5 -year DFS was $46.2 \%$ in patients with $\geq 10$ axillary LNMs. ${ }^{15}$ In a similar cohort of patients with $\geq 10$ axillary LNMs, Turker et al. demonstrated the highest 5-year DFS rate of $49.2 \%$ in patients with ER/PR+ and Her2-subtype. ${ }^{16}$ These results confirm our findings concerning DFS in patients with pN3a based on $\geq 10$ axillary LNMs, which was $48.8 \%$.

The definition of an infraclavicular (level III) lymph node during surgery may be open to interpretation. According to the American Society of Breast Surgeons, a level III ALND (extending to the apex of the axilla) is only recommended in patients with evidence of suspicious nodes located behind the pectoralis minor muscle (level II). ${ }^{17}$ However, in some cases a suspicious level II node can be incorrectly defined as infraclavicular (level III) node during ALND. As a consequence, these patients were considered $\mathrm{pN}_{3}$ a based on infraclavicular LNM 
rather than potentially $\mathrm{pN} 2 \mathrm{a}$ or $\mathrm{pNia}$, depending on the total number of axillary LNMs.

Due to new imaging techniques, the detection of infraclavicular LNM has increased over time. Prior to the introduction of the sixth edition of TNM in 2002, infraclavicular LNMs were detected during physical examination and/or surgery. In the current era, with imaging modalities like breast magnetic resonance imaging (MRI) and positron-emission tomography/computed tomography (PET/CT), smaller (infraclavicular) LNMs can be detected prior to surgery. ${ }^{18,19}$ However, the seventh edition of TNM is still based on a 2001 study in which infraclavicular LNMs were detected with physical examination and ultrasound rather than MRI or PET-CT., ${ }^{2,6}$ Our cohort consisted of patients diagnosed between 2005 and 2008, which is more in line with the current imaging modalities. MRI was already recommended in our study cohort according to the national guidelines of 2005 . $^{7,10}$

Although the incidence of patients with pN3a based on at least an infraclavicular LNM in our cohort is small (4.6\%), our findings suggest that reclassification in the next TNM classification should be considered. We advise to redefine an infraclavicular LNM as equivalent to other axillary LNMs rather than taking the location of infraclavicular LNM into account. Consequently, patients with an infraclavicular LNM with $\leq 9$ LNMs will be considered $\mathrm{pN} 2 \mathrm{a}$ rather than pN3a. In this way, infraclavicular LNM will become consistent with intramammary and interpectoral LNM, which are coded as axillary LNMs (level I/II) in the current TNM classification. ${ }^{7}$ Yet, adjuvant (radiation) therapy of infraclavicular LNM is still recommended.

This study had limitations. A major limitation of this study concerns the subgroup of patients with $\geq 10$ axillary LNMs, which still might have infraclavicular LNM as well. Yet, the focus of this study was to compare prognosis between both subgroups, since the current TNM atlas considers both as one category. Our results should therefore be interpreted with this important limitation in mind.

A second limitation of this study was the use of a retrospective database. Some clinically relevant parameters were not present, for instance, radiation therapy fields and the presence of lymphovascular invasion of the tumour. As a consequence, irradiation of regional nodal fields is unknown in this study cohort, which is generally recommended in breast cancer patients with LNMs. $^{20}$ Furthermore, presence of lymphovascular invasion can have a negative effect on overall survival. ${ }^{21}$

Third, these data only contain patients treated in the Netherlands between 2005 and 2008. This might have influence on prognosis when these data would be extrapolated to cohorts in other countries. For instance, the 5-year survival rate of breast cancer patients in the Netherlands still is different when compared to Asian or South American countries. ${ }^{22}$ 
Fourth, despite the collection of nationwide data between 2005 and 2008 in the Netherlands, the subgroup of patients with $\mathrm{pN}_{3}$ a based on at least an infraclavicular LNM remained small $(\mathrm{n}=83)$. As a consequence, the number of variables for multivariable Cox proportional hazards regression was restricted due to the limited number of events. ${ }^{12}$ However, differences between both subgroups regarding tumour subtypes and adjuvant treatment were small, which means that it is unlikely that the difference in prognosis would be attributable to the difference in covariates between the two cohorts.

In conclusion, DFS and OS of patients staged as $\mathrm{pN}_{3}$ a based on at least an infraclavicular LNM is superior compared to patients with $\geq 10$ axillary LNMs. Therefore, reclassification of infraclavicular LNM in the next edition of TNM should be considered to classify an infraclavicular LNM with fewer than 10 LNMs to $\mathrm{pN} 2 \mathrm{a}$ rather than $\mathrm{pN} 3 \mathrm{a}$. 


\section{References}

1. UICC, Committee on Clinical Stage Classification and Applied Statistics. Clinical stage classification and presentation of results, malignant tumors of the breast and larynx. Paris: International Union Against Cancer; 1958.

2. Singletary SE, Greene FL, Breast Task F. Revision of breast cancer staging: the 6th edition of the TNM classification. Semin Surg Oncol 2003;21:53-9.

3. Sobin LH, Fleming ID. TNM classification of malignant tumors, fifth edition (1997). Union Internationale Contre le Cancer and the American Joint Committee on Cancer. Cancer 1997;80:1803-4.

4. Beenken SW, Urist MM, Zhang Y, Desmond R, Krontiras H, Medina H, et al. Axillary lymph node status, but not tumor size, predicts locoregional recurrence and overall survival after mastectomy for breast cancer. Ann Surg 2003;237:732-8. discussion 738-9.

5. Sobin L, Wittekind C. International union against cancer (UICC): TNM classification of malignant tumors 6th edition. New York: WilleyeLiss; 2002.

6. Newman LA, Kuerer HM, Fornage B, Mirza N, Hunt KK, Ross MI, et al. Adverse prognostic significance of infraclavicular lymph nodes detected by ultrasonography in patients with locally advanced breast cancer. Am J Surg 2001;181:313-8.

7. Sobin LH, Gospodarowicz MK, Wittekind C. TNM classification of malignant tumours. John Wiley \& Sons; 2011.

8. Gospodarowicz M, Mackillop W, O'Sullivan B, Sobin L, Henson D, Hutter RV, et al. Prognostic factors in clinical decision making: the future. Cancer 2001;91(8 Suppl.):1688-95.

9. Webber C, Gospodarowicz M, Sobin LH, Wittekind C, Greene FL, Mason MD, et al. Improving the TNM classification: findings from a 10-year continuous literature review. Int J Cancer 2014;135:371-8.

10. CBO Kwaliteitsinstituut voor de Gezondheidszorg. Vereniging van Integrale Kankercentra. Guideline 'Treatment of breast cancer' (Richtlijn 'Behandeling van het Mammacarcinoom'). 2005.

11. Kaplan EL, Meier P. Nonparametric estimation from incomplete observations. J Am Stat Assoc 1958;53:457-81.

12. Vittinghoff E, McCulloch CE. Relaxing the rule of ten events per variable in logistic and Cox regression. Am J Epidemiol 2007;165:710-8.

13. Veronesi U, Viale G, Rotmensz N, Goldhirsch A. Rethinking TNM: breast cancer TNM classification for treatment decisionmaking and research. Breast 2006;15:3-8.

14. Brito RA, Valero V, Buzdar AU, Booser DJ, Ames F, Strom E, et al. Long-term results of combined-modality therapy for locally advanced breast cancer with ipsilateral supraclavicular metastases: the University of Texas M.D. Anderson Cancer Center experience. J Clin Oncol 2001;19:628-33.

15. Koca E, Kuzan TY, Dizdar O, Babacan T, Sahin I, Ararat E, et al. Outcomes of locally advanced breast cancer patients with $\geq 10$ positive axillary lymph nodes. Med Oncol 2013;30:615.

16. Turker I, Arslan UY, Yazici O, Uyeturk U, Oksuzoglu B, Budakoglu B, et al. Prognostic factors in operated stage IIIC, pathological N3a breast cancer patients. Breast care (Basel, Switzerland) 2014;9:421-7.

17. American Society of Breast Surgeons. Performance and Practice guidelines for axillary lymph node dissection in breast cancer patients. Available from:

https://www.breastsurgeons.org/new_layout/about/statements/PDF_Statements/PerformancePr acticeGuidelines_ALND.pdf. Visited: 17-6-2016.

18. Kuijs VJ, Moossdorff M, Schipper RJ, Beets-Tan RG, Heuts EM, Keymeulen KB, et al. The role of MRI in axillary lymph node imaging in breast cancer patients: a systematic review. Insights Imaging 2015;6:203-15.

19. Koolen BB, Valdes Olmos RA, Vogel WV, Vrancken Peeters MJ, Rodenhuis S, Rutgers EJ, et al. Pre-chemotherapy 18F-FDG PET/CT upstages nodal stage in stage II-III breast cancer patients treated with neoadjuvant chemotherapy. Breast Cancer Res Treat 2013;141:249-54. 
20. Senkus E, Kyriakides S, Ohno S, Penault-Llorca F, Poortmans P, Rutgers E, et al. Primary breast cancer: ESMO clinical practice guidelines for diagnosis, treatment and follow-up. Ann Oncol 2015;26(Suppl. 5):v8-30.

21. Houvenaeghel G, Classe JM, Garbay JR, Giard S, Cohen M, Faure C, et al. Survival impact and predictive factors of axillary recurrence after sentinel biopsy. Eur J Cancer 2016;58:73-82.

22. Allemani C, Weir HK, Carreira H, Harewood R, Spika D, Wang XS, et al. Global surveillance of cancer survival 1995e2009: analysis of individual data for 25,676,887 patients from 279 population-based registries in 67 countries (CONCORD-2). Lancet 2015;385977-1010. 
Appendix 7.1

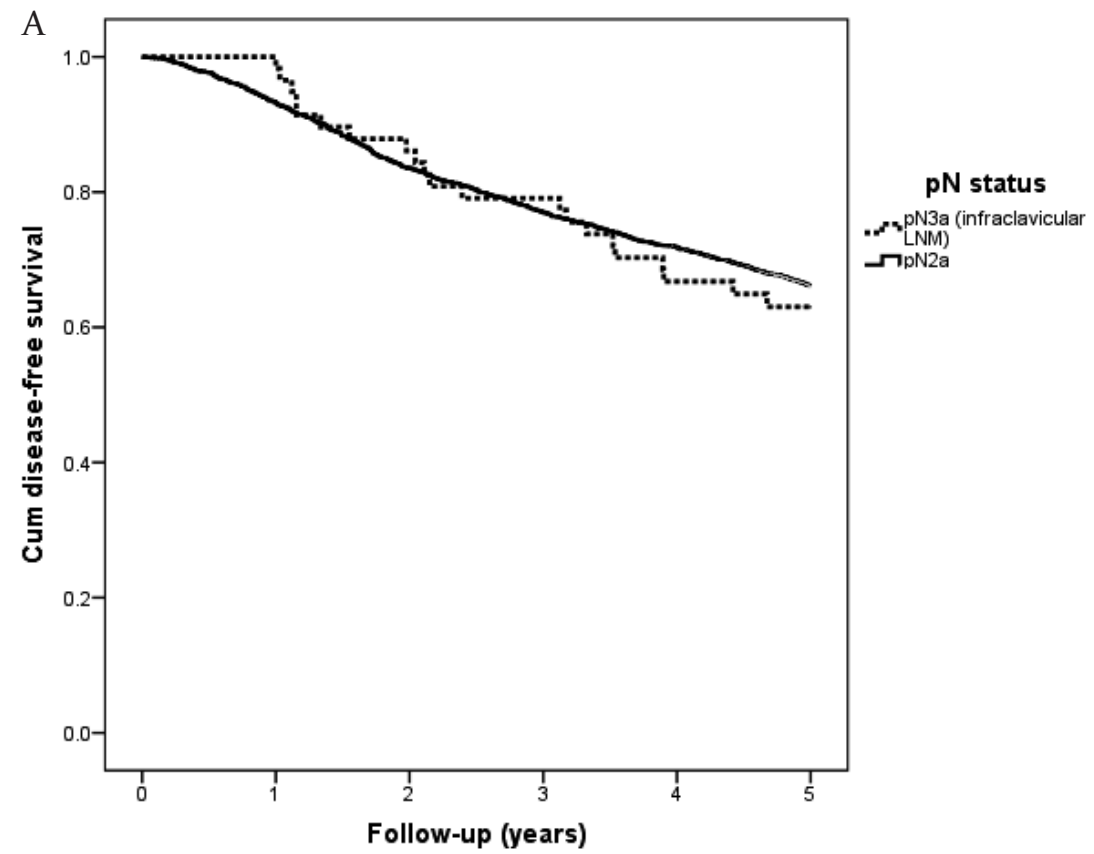

\begin{tabular}{lrrrrrr}
\hline $\begin{array}{l}\text { Infraclavicular } \\
\text { LNM }\end{array}$ & 58 & 57 & 49 & 45 & 37 & 23 \\
\hline pN2a & 2483 & 2317 & 2076 & 1916 & 1791 & 1670
\end{tabular}




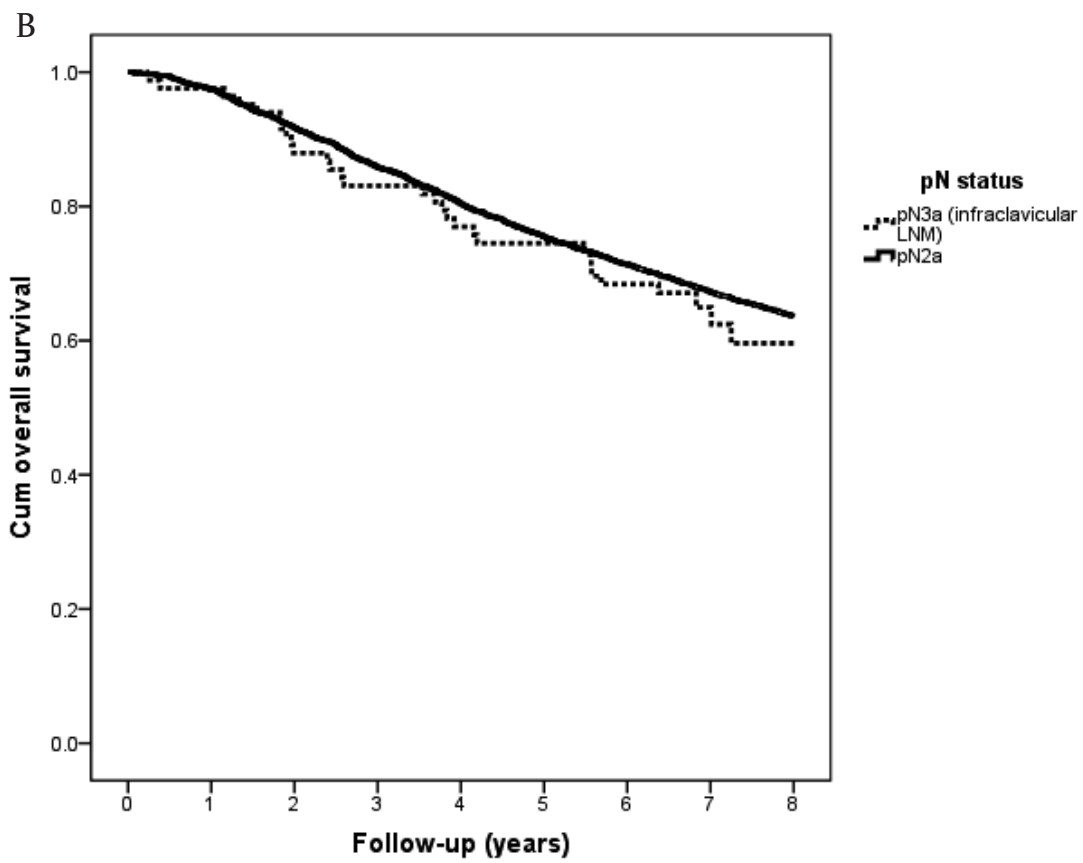

\begin{tabular}{lrrrrrrrrrr}
\hline $\begin{array}{l}\text { Infraclavicular } \\
\text { LNM }\end{array}$ & 83 & 81 & 73 & 68 & 63 & 61 & 56 & 26 & 12 \\
\hline pN2a & 1705 & 1607 & 1444 & 1288 & 1130 & 1004 & 912 & 627 & 373
\end{tabular}




\section{Appendix 7.2}

A

\begin{tabular}{lcccc}
\hline & \multicolumn{2}{c}{ Univariable analysis } & \multicolumn{2}{c}{ Multivariable analysis } \\
\cline { 2 - 5 } & HR $(95 \% \mathrm{CI})$ & $P$-value & HR $(95 \% \mathrm{CI})$ & $P$-value \\
\hline pN2a & Reference & & Reference & 0.491 \\
\hline Infraclavicular LNM & $1.10(0.71-1.70)$ & 0.661 & $1.17(0.76-1.80)$ & - \\
\hline Age (per year increment) & $1.03(1.02-1.03)$ & $<0.001$ & - & - \\
pT-stage T3-4 vs To-2 & $1.94(1.64-2.31)$ & $<0.001$ & - & - \\
\hline Tumour grade 3 vs 1-2 & $1.65(1.44-1.88)$ & $<0.001$ & $<0.001$ \\
Triple negative subtype Yes vs No & $2.92(2.47-3.46)$ & $<0.001$ & $3.08(2.60-3.65)$ & $<0.001$ \\
\hline Chemotherapy Yes vs No & $0.41(0.35-0.47)$ & $<0.001$ & $0.44(0.38-0.51)$ & - \\
Trastuzumab Yes vs No & $0.72(0.59-0.88)$ & 0.001 & - & - \\
\hline Endocrine therapy Yes vs No & $0.42(0.36-0.48)$ & $<0.001$ & & $<0.001$ \\
Radiation therapy Yes vs No & $0.40(0.33-0.49)$ & $<0.001$ & $0.52(0.43-0.64)$ & $<$ \\
\hline
\end{tabular}

Abbreviations: LNM lymph node metastases, $p T$-stage pathologic tumour stage.

B

\begin{tabular}{lcccc}
\hline & \multicolumn{2}{c}{ Univariable analysis } & \multicolumn{2}{c}{ Multivariable analysis } \\
\cline { 2 - 5 } pN2a & $\begin{array}{c}\text { HR }(95 \% \mathrm{CI}) \\
\text { Reference }\end{array}$ & $\begin{array}{c}\text { P-value } \\
\text { Infraclavicular LNM }\end{array}$ & $\begin{array}{c}\text { HR }(95 \% \mathrm{CI}) \\
\text { Reference }\end{array}$ & $P$-value \\
\hline Age (per year increment) & $1.13(0.79-1.63)$ & 0.501 & $1.25(0.87-1.79)$ & 0.233 \\
pT-stage T3-4 vs To-2 & $1.88(1.64-2.18)$ & $<0.001$ & $1.82(1.58-2.10)$ & $<$ \\
\hline Tumour grade 3 vs 1-2 & $1.50(1.34-1.68)$ & $<0.001$ & - & - \\
Triple negative subtype Yes vs No & $2.68(2.32-3.10)$ & $<0.001$ & $1.75(1.47-2.10)$ & $<0.001$ \\
\hline Chemotherapy Yes vs No & $0.31(0.28-0.35)$ & $<0.001$ & $0.34(0.30-0.38)$ & $<0.001$ \\
Trastuzumab Yes vs No & $0.62(0.52-0.74)$ & $<0.001$ & - & - \\
\hline Endocrine therapy Yes vs No & $0.44(0.39-0.49)$ & $<0.001$ & $0.51(0.45-0.59)$ & $<0.001$ \\
Radiation therapy Yes vs No & $0.34(0.30-0.40)$ & $<0.001$ & $0.56(0.48-0.65)$ & $<0.001$ \\
\hline
\end{tabular}

Abbreviations: LNM lymph node metastases, $p T$-stage pathologic tumour stage. 


\section{Chapter}

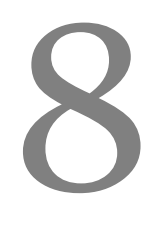

Prognosis of residual axillary disease after neoadjuvant chemotherapy in clinically nodepositive breast cancer patients: isolated tumour cells and micrometastases carry a better prognosis than macrometastases

TJA van Nijnatten*, JM Simons*, M Moossdorff, L de Munck, MBI Lobbes, CC van der Pol, LB Koppert, EJT Luiten, ML Smidt

* Contributed equally tot his work Breast Cancer Res Treat 2017;163:159-166 


\section{Abstract}

\section{Purpose}

The aim of this study was to compare disease-free survival (DFS) and overall survival (OS) between clinically node-positive breast cancer patients, treated with neoadjuvant chemotherapy (NAC), with axillary pathologic complete response (ypNo), residual axillary isolated tumour cells or micrometastases (ypNitc/mi), and residual axillary macrometastases (ypNi-3).

\section{Methods}

All patients diagnosed with clinically node-positive primary invasive breast cancer treated with NAC and subsequent axillary lymph node dissection between 2005 and 2008 were retrospectively analyzed. Data were obtained from the Netherlands Cancer Registry. Patients were stratified by final pathological axillary status: ypNo, ypNitc/mi, or ypNi-3. The main outcome measures DFS and OS were analyzed using Kaplan-Meier survival analysis. Uni- and multivariable cox regression analyses were used to determine independent predictors for DFS and OS.

\section{Results}

A total of 1347 patients were included. Pathologic nodal status was ypNo in $22.2 \%$, ypNitc/mi in 3.8\%, and ypN1-3 in $74.0 \%$ of patients. Overall, 5 -year DFS was $57.8 \%$ and mean OS was 7.4 years. DFS and OS were comparable between ypNo and ypNitc/mi (HR 1.38 (o.40-4.79, $P=0.613$ ) and HR 0.92 (o.27-3.09, $P=0.889$ ), respectively), but significantly different between ypNo and ypN1-3 (HR 1.78 (1.063.00, $P=0.031)$ and HR 1.70 (1.07-2.71, $P=0.026)$, respectively).

\section{Conclusions}

Clinically node-positive patients, treated with NAC, with axillary nodal status ypNo or ypNitc/mi carry similar prognosis regarding DFS and OS. Axillary nodal status ypN1-3 is associated with a less favourable prognosis. Future studies should consider ypNo and ypNitc/mi as one entity. 


\section{Introduction}

Over the past 20 years, a trend toward a less invasive approach regarding the surgical management of the axilla in breast cancer patients has been observed. Nowadays, a sentinel lymph node biopsy (SLNB) has been widely adopted for staging of early-stage clinically node-negative breast cancer. ${ }^{1}$ In case of a sentinel lymph node (SLN) containing isolated tumour cells (ITCs) or micrometastases, a completion of axillary lymph node dissection (ALND) does not improve survival, nor does it reduce regional recurrence. Consequently, ALND following SLNB has been abandoned in these patients. ${ }^{2-4}$ The ACOSOG Zoo11 trial demonstrated no significant effect on prognosis when ALND is omitted in case of a SLN containing a limited number of metastases, even macrometastases, in patients treated with breast conserving therapy. ${ }^{3}$

In clinically node-positive $(\mathrm{cN}+)$ patients, ALND is regarded as standard surgical therapy. However, increased utilization of neoadjuvant chemotherapy (NAC) results in axillary pathologic complete response (pCR) in $30-40 \%$ of patients. ${ }^{5}$ Consequently, the value of ALND is topic of debate. Various studies demonstrated that axillary pCR after NAC is associated with improved prognosis. $^{6-8}$ Residual axillary disease has a less favourable prognosis, but it is unknown whether different degrees of residual axillary disease (i.e., ITCs, micrometastases, macrometastases) all have similar prognosis.

Hence, the purpose of this study was to compare prognosis of axillary pCR, residual ITCs, or micrometastases and residual macrometastases in $\mathrm{cN}+$ patients treated with NAC.

\section{Methods}

\section{Data collection}

In this study, all pathologically confirmed $\mathrm{cN}+$ patients diagnosed with primary invasive breast cancer and treated with NAC (with or without immunotherapy) followed by ALND between 2005 and 2008 were included. Exclusion criteria were synchronous breast cancer, primary surgical treatment, neoadjuvant radiation therapy, neoadjuvant endocrine therapy, unknown pathological nodal status, and distant metastases diagnosed within 91 days after primary breast cancer diagnosis. Patients who did not undergo ALND were also excluded.

Data were obtained from the Netherlands Cancer Registry (NCR), which is managed by the Netherlands Comprehensive Cancer Organisation (NCCO). The PALGA foundation (Pathologisch-Anatomisch Landelijk Geautomatiseerd Archief), a nationwide network and registry of histopathology and cytopathology 
diagnosis in the Netherlands, regularly submits reports of all diagnosed malignancies to the cancer registry. After notification, trained data collection registrars from the NCR extracted data from patients' records. Data were collected on age, tumour type, receptor status, surgical procedures, systemic therapy, adjuvant radiation therapy, and pathology results, including pathological TNM stage and tumour grade. During a 5-year period after initial diagnosis, the first of the following breast cancer events was registered: any local, regional, or contralateral recurrence or distant metastasis. Date of death or date of emigration was derived from the Municipal Personal Records Database (Basisregistratie Personen, BRP) and files until December 31, 2014 were analyzed. Patients were stratified into three subgroups according to final pathologic axillary nodal status after completion of NAC and definitive surgery: pCR (ypNo), residual isolated tumour cells or micrometastases (ypNitc/mi), and residual macrometastases (ypN1-3).

\section{Neoadjuvant chemotherapy (NAC) with/without immunotherapy regimen}

During the study period, the Dutch national guideline of 2005 was in use. ${ }^{9}$ This guideline recommended chemotherapy regimens consisting of five courses 5 Fluorouracil, Epirubicin, Cyclophosphamide (FEC), or six courses of Taxotere, Adriamycin, and Cyclophosphamide (TAC). In case of Her2Neu receptor (Her2) amplification, targeted therapy (trastuzumab) was recommended in addition to chemotherapy.

\section{Statistics}

Statistical analyses were performed using Statistical Package for the Social Sciences software (Version 22, IBM, Armonk, New York, USA). General characteristics between the three subgroups were compared using Chi squared test for categorical data and One-way ANOVA for continuous data, after confirmation of Levene's test for equality of variances. If Levene's test demonstrated significant differences among the population variances, KruskallWallis test was used.

DFS was defined as time from diagnosis to any local (including carcinoma in situ), regional, or contralateral recurrence, distant metastasis or mortality within 5 years after the primary diagnosis. Events occurring o-91 days after diagnosis were considered synchronous to the original tumour and were not counted as recurrence. OS was defined as the time interval between date of diagnosis and date of death, date of first event, date of last follow-up, or date of emigration.

DFS and OS for the three subgroups were calculated with Kaplan-Meier curves and compared with the log-rank test. $P$ values (two-sided) $<0.05$ were 
considered statistically significant. Relevant clinicopathological variables associated with DFS and OS were examined using univariable and, where applicable, multivariable Cox proportional hazards regression, with Hazard Ratio (HR) and corresponding 95\% confidence intervals.

\section{Results}

Between 2005 and 2008, 8176 patients were diagnosed with $\mathrm{cN}+$ breast cancer in the Netherlands. Patients were excluded for several reasons: 6553 patients underwent primary surgery; 204 patients did not undergo ALND; 9 patients were treated with neoadjuvant radiotherapy; 61 patients were treated with neoadjuvant endocrine therapy; and ypN status was unknown for 11 patients (Figure 8.1). A final total of 1347 patients were included for this study: $299 \mathrm{ypNo}, 51 \mathrm{ypNitc} / \mathrm{mi}$ and $997 \mathrm{ypN}^{\mathrm{p}-3}$.

The incidence of $\mathrm{pCR}$ of the primary tumour was higher in patients with ypNo compared to ypNitc/mi and ypN1-3 patients $(41.1 \%$ vs. $19.6 \%$ and $7.1 \%$, respectively, $P<0.001)$. Furthermore, lobular carcinoma was observed more often in patients with ypN1-3 than in ypNo and ypNitc/mi patients (9.4\% vs. $5.4 \%$ and $3.9 \%$, respectively, $P=0.039)$. Adjuvant radiation therapy was applied more often in $\mathrm{ypN}_{1}-3$ as compared to ypNo and ypNitc/mi patients $(92.1 \%$ vs. $80.9 \%$ and $80.4 \%$, respectively, $P<0.001$, Table 8.1 ).

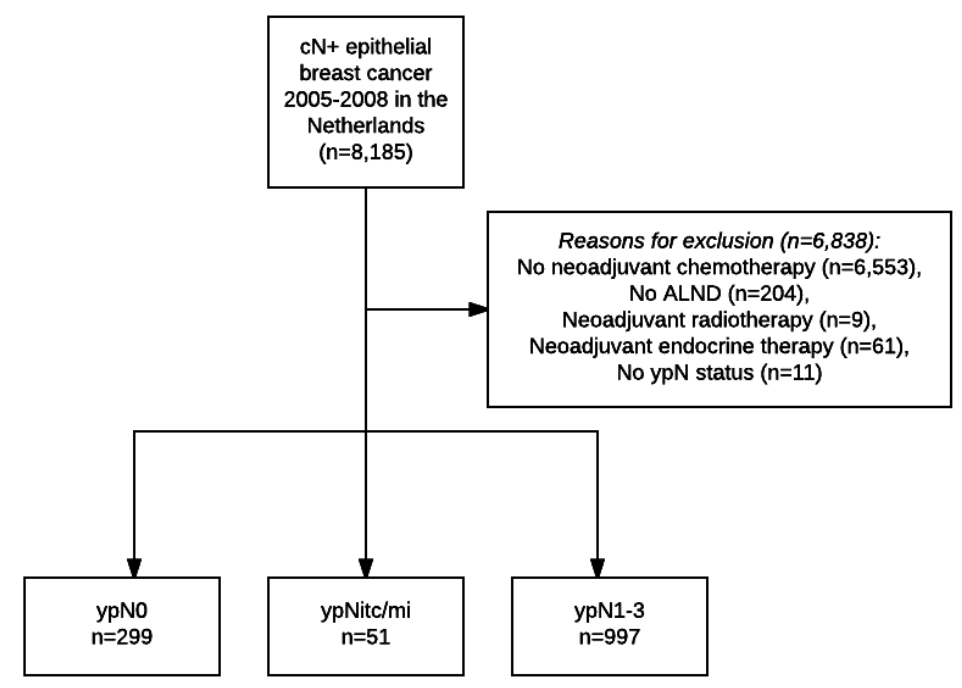

Figure 8.1 Flowchart of included patients. Abbreviations: $c N+$ clinically node positive status, SLNB sentinel lymph node biopsy, ALND axillary lymph node dissection, ypNO axillary pathologic complete response, ypNitc/mi axillary residual isolated tumour cells or micrometastases, ypN1-3 axillary residual macrometastases. 
Table 8.1 General characteristics.

\begin{tabular}{|c|c|c|c|c|}
\hline & $\begin{array}{c}y p N o \\
(n=299)\end{array}$ & $\begin{array}{c}y p N i t c / m i \\
(n=51)\end{array}$ & $\begin{array}{l}y p N_{1-3} \\
(n=997)\end{array}$ & P-value \\
\hline Mean age (years) (range) & $\begin{array}{c}48.9 \\
(27-77)\end{array}$ & $\begin{array}{c}48.2 \\
(29-81)\end{array}$ & $\begin{array}{c}50.4 \\
(22-85)\end{array}$ & 0.053 \\
\hline \multicolumn{5}{|l|}{ Clinical T-stage (\%) } \\
\hline cTo-is & $1(0.3)$ & o & $5(0.5)$ & 0.826 \\
\hline cT1-2 & $138(47.0)$ & $26(52.0)$ & $458(47.0)$ & 0.780 \\
\hline $\mathrm{cT}_{3}-4$ & $153(52.0)$ & $24(48.0)$ & $509(52.0)$ & 0.853 \\
\hline cTx & 7 & 1 & 25 & - \\
\hline \multicolumn{5}{|l|}{ Pathologic T-stage (\%) } \\
\hline ypTo-is & $123(50.8)$ & $10(26.3)$ & $71(8.5)$ & $<0.001$ \\
\hline ypT1-2 & $107(44.2)$ & $25(65.8)$ & $570(68.5)$ & $<0.001$ \\
\hline урТ $3-4$ & $12(5.0)$ & $3(7.9)$ & $191(23.0)$ & $<0.001$ \\
\hline Unknown & 57 & 13 & 165 & - \\
\hline \multicolumn{5}{|l|}{ Tumour grade (\%) } \\
\hline $1-2$ & $28(31.5)$ & $7(50.0)$ & $197(43.9)$ & $<0.001$ \\
\hline 3 & $61(68.5)$ & $7(50.0)$ & $252(56.1)$ & 0.051 \\
\hline Unknown & 210 & 37 & 548 & - \\
\hline \multicolumn{5}{|l|}{ Tumour type (\%) } \\
\hline Ductal & $227(75.9)$ & $41(80.4)$ & $746(74.8)$ & 0.739 \\
\hline Lobular & $16(5.4)$ & $2(3.9)$ & $94(9.4)$ & 0.039 \\
\hline Other $^{\mathrm{a}}$ & $56(18.7)$ & $8(15.7)$ & $157(15.8)$ & 0.470 \\
\hline \multicolumn{5}{|l|}{ Subtype (\%) } \\
\hline $\mathrm{ER}+\mathrm{PR}+$, Her2- & $35(12.8)$ & $11(22.5)$ & $349(37 \cdot 3)$ & $<0.001$ \\
\hline ER+PR-, Her2- & $21(7.7)$ & $7(14 \cdot 3)$ & $122(13.0)$ & 0.035 \\
\hline ER+Her2+ & $47(17.1)$ & $20(40.8)$ & $152(16.3)$ & $<0.001$ \\
\hline ER-Her2+ & $97(35.4)$ & $5(10.2)$ & $146(15.6)$ & $<0.001$ \\
\hline Triple negative & $74(27.0)$ & $6(12.2)$ & $166(17.8)$ & 0.003 \\
\hline Unknown & 25 & 2 & 62 & - \\
\hline \multicolumn{5}{|l|}{ Breast surgery (\%) } \\
\hline Breast conserving therapy & $62(20.7)$ & $12(23.5)$ & $181(18.2)$ & 0.421 \\
\hline Mastectomy & $237(79 \cdot 3)$ & $39(76.5)$ & $816(81.8)$ & 0.421 \\
\hline Unknown & o & o & 1 & - \\
\hline \multicolumn{5}{|l|}{ Radiation therapy (\%) } \\
\hline Yes & $242(80.9)$ & $41(80.4)$ & $918(92.1)$ & $<0.001$ \\
\hline \multicolumn{5}{|c|}{ Endocrine therapy to ER+ subtype (\%) } \\
\hline Yes & $95(84.8)$ & $35(92.1)$ & $600(91.5)$ & 0.080 \\
\hline \multicolumn{5}{|c|}{ Trastuzumab to Her2+ subtype (\%) } \\
\hline Yes & $127(92.0)$ & $19(76.0)$ & $247(89.2)$ & 0.057 \\
\hline
\end{tabular}

${ }^{a}$ Including adenocarcinoma not otherwise specified, mucinous carcinoma, and mixed carcinoma.

Abbreviations: $y p N o$ axillary pathologic complete response, $y p N i t c / m i$ axillary residual isolated tumour cells or micrometastases, $y \mathrm{pN}_{1-3}$ axillary residual macrometastases, $c T$-stage clinical tumour stage, $p T$-stage pathologic tumour stage, ER estrogen, $P R$ progesterone, Her human epidermal growth factor receptor 2.

\section{Disease-free survival}

Five-year follow-up was available for 944 patients (70.1\%; $\mathrm{n}=206$ ypNo, $\mathrm{n}=34$ ypNoi+/ypNimi, $\mathrm{n}=704 \mathrm{ypN} 1-3)$ : Recurrence occurred in 377 patients (39.9\%) and 22 patients died within 5 years $(2.3 \%)$. This resulted in a DFS event in $42.2 \%$ of the 
patients. DFS did not differ significantly between ypNo and ypNitc/mi (71.8 vs. $70.6 \%, P=0.978)$. When DFS was compared between ypNo and ypN1-3, a significant difference was found (71.8 vs. 53.4\%; $P=0.049$ ) (Figure 8.2a).

Multivariable Cox regression analyses demonstrated no significant difference in DFS between ypNo and ypNitc/mi (HR 1.38 (o.40-4.79), $P=0.613$ ), but a significant difference in DFS between ypNo and ypN1-3 (HR 1.78 (1.06-3.0o), $P=$ o.031) (Table 8.2).

Furthermore, higher ypT stage (ypT 1-2: HR 2.73 (1.39-5.39), $P=0.004$ and ypT 3-4: HR 4.71 (2.35-9.43), P<0.0o1) and higher tumour grade (HR 1.69 (1.19-2.40), $P=0.004)$ were identified as independent predictors of decreased DFS, whereas endocrine therapy was identified as independent predictor of increased DFS (HR 0.55 (0.36-0.85), $P=0.007$ ).

A

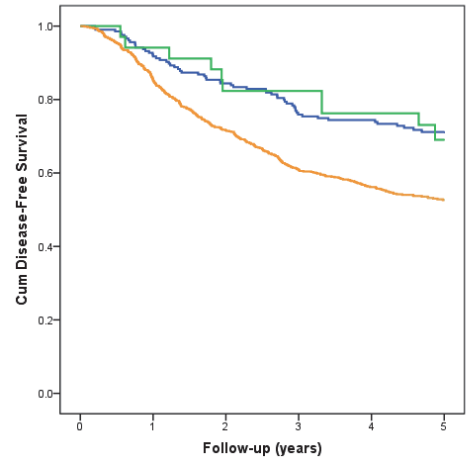

\begin{tabular}{|l|r|r|r|r|r|r|}
\hline$y p N O$ & 206 & 188 & 172 & 152 & 146 & 80 \\
\hline$y p N i t c / m i$ & 34 & 32 & 28 & 27 & 25 & 15 \\
\hline$y p N I-3$ & 704 & 598 & 499 & 423 & 381 & 241 \\
\hline
\end{tabular}

B

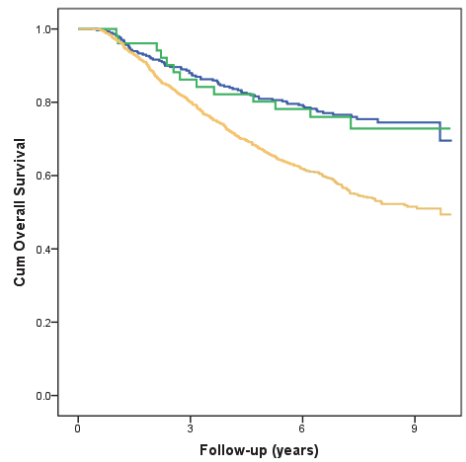

\begin{tabular}{|l|r|r|r|r|}
\hline$y p N O$ & 299 & 263 & 236 & 43 \\
\hline$y p N i t c / m i$ & 51 & 43 & 38 & 9 \\
\hline$y p N 1-3$ & 997 & 795 & 612 & 109 \\
\hline
\end{tabular}

Figure 8.2 Kaplan-Meier curves for disease-free (A) and overall survival (B), including number at risk. Abbreviations: $y p N$ status pathologic nodal status after neoadjuvant chemotherapy, ypNO axillary pathologic complete response, ypNitc/mi axillary residual isolated tumour cells or micrometastases, ypN1-3 axillary residual macrometastases.

\section{Overall survival}

Mean OS was 7.4 years (range $0.4-10$ years): 8.3 years for ypNo, 8.2 years for ypNitc/mi, and 7.0 years for ypN1-3 (Figure 8.2b). OS was comparable between ypNo and ypNitc/mi $(P=0.875)$. However, OS was significantly lower for ypN1-3 as compared to ypNo $(P=0.014)$. 
Table 8.2 Uni- and multivariable analyses of predictors for disease-free survival at 5 years.

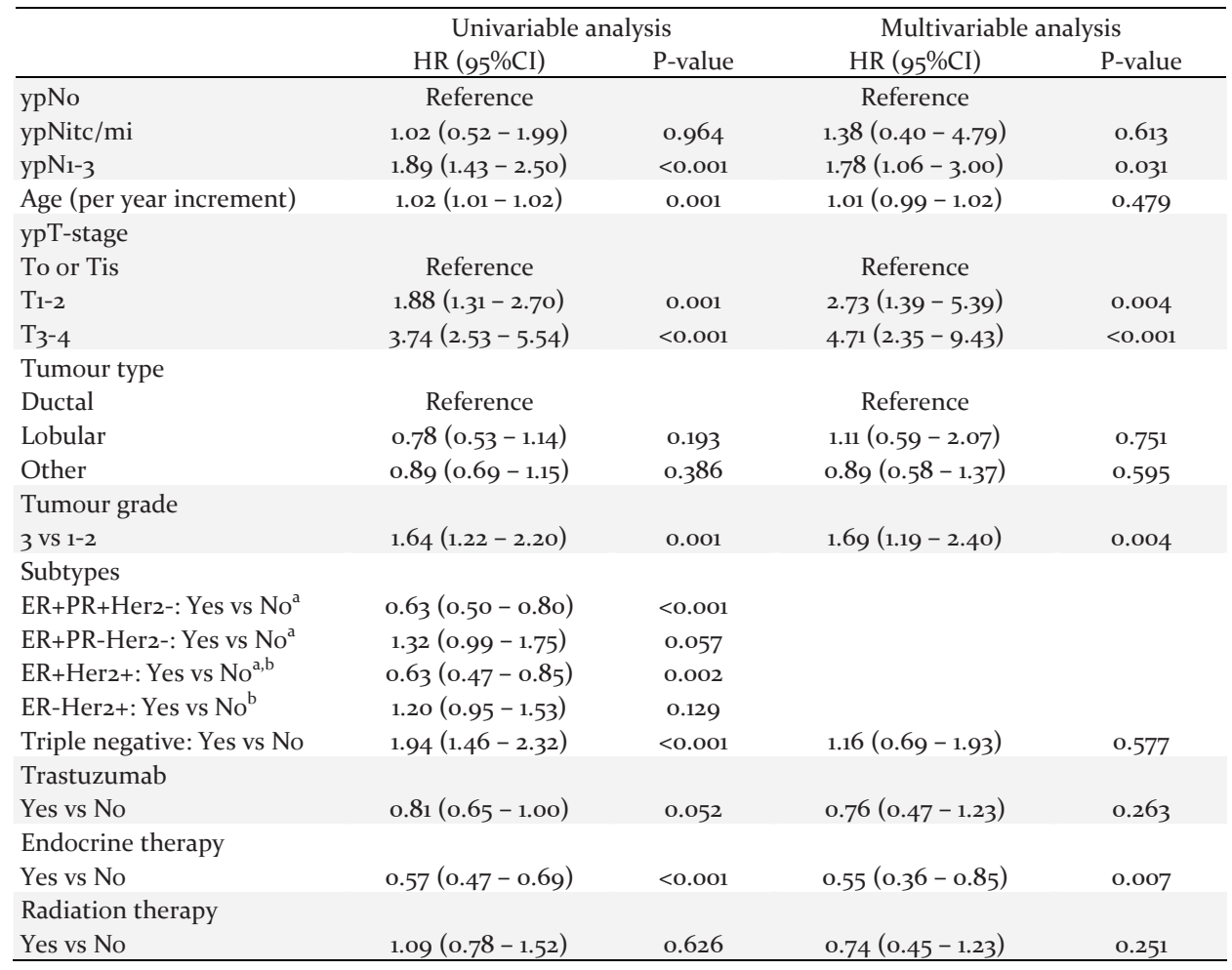

a Excluded from multivariable analysis due to collinearity with endocrine therapy. ${ }^{\text {b }}$ Excluded from multivariable analysis due to collinearity with trastuzumab.

Abbreviations: HR Hazard ratio, ypNo axillary pathologic complete response, ypNitc/mi axillary residual isolated tumour cells or micrometastases, ypN1-3 axillary residual macrometastases, ypT-stage pathologic tumour stage after neo-adjuvant chemotherapy, ER estrogen, $P R$ progesterone, Her2 human epidermal growth factor receptor 2 .

Multivariable Cox regression analyses demonstrated no significant difference in OS between ypNo and ypNitc/mi (HR: 0.92 (o.27-3.09), $P=0.889$ ), but a significant difference in OS between ypNo and ypN1-3 (HR 1.70 (1.07-2.71), $P=$ o.026) (Table 8.3).

Other independent predictors of decreased OS were higher ypT stage ((ypT 1-2: HR 2.40 (1.32-4.36), $P=0.004)$ and (ypT 3-4: HR 4.38 (2.37-8.12), $P<0.001)$ ) and higher tumour grade (HR $1.72(1.25-2.36), P=0.001)$. Furthermore, endocrine therapy (HR $0.49(0.34-0.72), P<0.001)$ was identified as an independent predictor of increased OS. 
Table 8.3 Uni- and multivariable analyses of predictors for overall survival.

\begin{tabular}{|c|c|c|c|c|}
\hline & \multicolumn{2}{|c|}{ Univariable analysis } & \multicolumn{2}{|c|}{ Multivariable analysis } \\
\hline & $\mathrm{HR}(95 \% \mathrm{CI})$ & $\mathrm{P}$-value & $\mathrm{HR}(95 \% \mathrm{CI})$ & $\mathrm{P}$-value \\
\hline ypNo & Reference & & Reference & \\
\hline ypNitc/mi & $1.07(0.59-1.94)$ & 0.816 & $0.92(0.27-3.09)$ & 0.889 \\
\hline ypN1-3 & $2.07(1.62-2.67)$ & $<0.001$ & $1.70(1.07-2.71)$ & 0.026 \\
\hline Age (per year increment) & $1.02(1.02-1.03)$ & $<0.001$ & $1.01(1.00-1.02)$ & 0.082 \\
\hline \multicolumn{5}{|l|}{ ypT-stage } \\
\hline To or Tis & Reference & & Reference & \\
\hline $\mathrm{T}_{1-2}$ & $1.60(1.19-2.15)$ & 0.002 & $2.40(1.32-4.36)$ & 0.004 \\
\hline $\mathrm{T}_{3}-4$ & $3.33(2.41-4.59)$ & $<0.001$ & $4.38(2.37-8.12)$ & $<0.001$ \\
\hline \multicolumn{5}{|l|}{ Tumour type } \\
\hline Ductal & Reference & & Reference & \\
\hline Lobular & $0.98(0.72-1.33)$ & 0.906 & $1.33(0.78-2.27)$ & 0.288 \\
\hline Other & $0.98(0.78-1.24)$ & 0.882 & $1.03(0.69-1.53)$ & 0.887 \\
\hline \multicolumn{5}{|l|}{ Tumour grade } \\
\hline 3 Vs $1-2$ & $1.84(1.41-2.40)$ & $<0.001$ & $1.72(1.25-2.36)$ & 0.001 \\
\hline \multicolumn{5}{|l|}{ Subtypes } \\
\hline $\mathrm{ER}+\mathrm{PR}+\mathrm{Her2}-:$ Yes vs $\mathrm{No}^{\mathrm{a}}$ & $0.62(0.51-0.76)$ & $<0.001$ & & \\
\hline ER+PR-Her2-: Yes vs No ${ }^{a}$ & $1.28(1.00-1.65)$ & 0.050 & & \\
\hline $\mathrm{ER}+\mathrm{Her} 2+$ : Yes vs $\mathrm{No}^{\mathrm{a}, \mathrm{b}}$ & $0.58(0.43-0.73)$ & $<0.001$ & & \\
\hline ER-Her2+: Yes vs No ${ }^{\mathrm{b}}$ & $1.15(0.93-1.42)$ & 0.201 & & \\
\hline Triple negative: Yes vs No & $2.10(1.73-2.56)$ & $<0.001$ & $1.38(0.90-2.13)$ & 0.145 \\
\hline \multicolumn{5}{|l|}{ Trastuzumab } \\
\hline Yes vs No & $0.72(0.59-0.88)$ & 0.001 & $0.70(0.46-1.07)$ & 0.101 \\
\hline \multicolumn{5}{|l|}{ Endocrine therapy } \\
\hline Yes vs No & $0.56(0.47-0.66)$ & $<0.001$ & $0.49(0.34-0.72)$ & $<0.001$ \\
\hline \multicolumn{5}{|l|}{ Radiation therapy } \\
\hline Yes vs No & $1.31(0.96-1.77)$ & 0.086 & $1.06(0.64-1.74)$ & 0.829 \\
\hline
\end{tabular}

a Excluded from multivariable analysis due to collinearity with endocrine therapy. ${ }^{\text {b }}$ Excluded from multivariable analysis due to collinearity with trastuzumab.

Abbreviations: HR Hazard Ratio, ypNo axillary pathologic complete response, ypNitc/mi axillary residual isolated tumour cells or micrometastases, ypN1-3 axillary residual macrometastases, ypT-stage pathologic tumour stage after neo-adjuvant chemotherapy, $E R$ estrogen, $P R$ progesterone, Herz human epidermal growth factor receptor 2 .

\section{Discussion}

This is the first study comparing prognosis of ypNo with ypNitc/mi and ypN1-3 in $\mathrm{cN}+$ breast cancer patients treated with NAC. It is well known that axillary pCR is an important prognostic factor. ${ }^{6-8}$ Residual axillary disease after completion of NAC is associated with a less favourable prognosis. However, to our knowledge, this is the first study that compares the long-term effect of different degrees of residual disease on prognosis. Our study showed that ypNo and ypNitc/mi carry similar prognosis and that ypN1-3 carries a significantly different and less favourable prognosis in terms of DFS and OS. 
Current guidelines still recommend to perform ALND in $\mathrm{cN}+$ patients following NAC irrespective of axillary response. ${ }^{10,11}$ However, $\mathrm{cN}+$ patients converting to axillary $\mathrm{pCR}$ after completion of NAC remain a topic of debate since they are not expected to benefit from ALND. A non-invasive technique to accurately diagnose $\mathrm{pCR}$ is currently unavailable. Various minimally invasive procedures have been suggested for this purpose. The SLNB was studied extensively and its reliability seems questionable with a reported overall false negative rate (FNR) of $15.1 \%$ and negative predictive values (NPV) of $86 \%$ or lower. ${ }^{5}$ Other recently introduced minimally invasive techniques, the MARI procedure (Marking the Axillary lymph node with Radioactive Iodine seeds) and TAD (Targeted Axillary Dissection), are promising with FNRs of 7 and $2 \%$, respectively. However, with only evidence available of single center studies comprising small cohorts that support these techniques it is not (yet) safe to implement them in clinical practice. ${ }^{12,13}$

In our cohort, all patients underwent an ALND and thus our results do not directly support a change in surgical axillary treatment after the completion of NAC. Considering the comparable prognosis between ypNo and ypNitc/mi, our results do question whether ypNitc/mi may mimic ypNo more than residual axillary disease. Thus, when minimally invasive procedures prove to predict the status of the axilla accurately, the indications for omitting ALND may not just be limited to ypNo. Therefore, current research on reducing axillary management in $\mathrm{cN}+$ patients should not focus only on ypNo patients, but also on patients with ypNitc/mi. In future, ALND may be rendered as a procedure only to manage residual macrometastases.

In clinically node-negative patients in adjuvant setting, the SLNB with a relatively high FNR of about $8 \%$ is permitted since axillary recurrences are rare and previous studies have shown that not all axillary residual disease eventually converts to clinically overt disease. ${ }^{2,3,14}$ This is in part effectuated by adjuvant therapy (i.e., radiation and/or systemic therapy) and by biological subtypes influencing recurrence patterns. In $\mathrm{cN}+$ patients, however, no studies have adequately evaluated prognostic impact of omitting ALND in case of residual axillary disease. Despite this, a trend toward replacing ALND by less invasive axillary staging procedures that are known to miss potentially therapy-resistant disease is already ongoing worldwide. Therefore, it is of utmost importance to prospectively collect data of these patients to detect potential influences on prognosis.

Since prognosis seems comparable between post-ALND ypNo and ypNitc/mi in $\mathrm{cN}+$ patients treated with NAC, imaging might play an important role in axillary staging after NAC in the future. Since ITCs and micrometastases are not detectable on high-resolution exams, such as MRI or 18F-FDG PET/CT, imaging techniques were considered inaccurate for nodal assessment after completion of 
NAC. Yet, with our current observations in mind, dedicated axillary imaging is reentering the arena as a modality to non-invasively identify residual macrometastases rather than 'any' extent of residual disease (including ITCs and micrometastases).

The strength of the current study is the large cohort of patients that all underwent ALND after NAC. But our study also has several limitations. Subgroups ypNo and ypN1-3 comprised 299 and 997 patients, respectively, where subgroup ypNitc/mi comprised only 51 patients. Our ypNitc/mi subcohort was too small to explore the influence of single versus multiple tumour-positive lymph nodes on prognosis, and further studies are needed to explore this concept. Yet, this subset of patients can be considered unique since ypNitc/mi in $\mathrm{cN}+$ breast cancer is rare and a previously reported study included only a few ypNitc/mi patients. ${ }^{15}$

Furthermore, our cohort was treated up to a decade ago. In that time frame, different guidelines were effective, and therefore results should be interpreted carefully regarding current practice. For example, some Her2+ patients did not receive trastuzumab in our cohort (19.6\%), since trastuzumab was just introduced by that time.

Finally, our results are based on a retrospective study design. Consequently, details on additional radiation therapy could not be taken into account since radiation therapy fields were not recorded for each patient. Therefore, its influence on prognosis could not be explored in more detail.

In conclusion, our study showed that prognosis of $\mathrm{cN}+$ patients who receive NAC is affected by the degree of axillary residual disease as measured in ALND specimens. Prognosis of isolated tumour cells and micrometastases was comparable to prognosis of ypNo and more favorable than prognosis of macrometastases in terms of DFS and OS irrespective of tumour type. Ongoing and future studies should therefore consider ypNo and ypNitc/mi as one entity. Future research must explore which patients may safely receive a different, less invasive approach than the current standard of performing ALND after completion of NAC in all patients that were $\mathrm{cN}+$ prior to NAC. 


\section{References}

1. Pilewskie ML, Morrow M. Management of the clinically node-negative axilla: what have we learned from the clinical trials? Oncology (Williston Park) 2014;28(5):371-8.

2. Galimberti V, Cole BF, Zurrida S, Viale G, Luini A, Veronesi P, Baratella P, Chifu C, Sargenti M, Intra M, Gentilini O, Mastropasqua MG, Mazzarol G, Massarut S, Garbay JR, Zgajnar J, Galatius H, Recalcati A, Littlejohn D, Bamert M, Colleoni M, Price KN, Regan MM, Goldhirsch A, Coates AS, Gelber RD, Veronesi U, International Breast Cancer Study Group Trial i. Axillary dissection versus no axillary dissection in patients with sentinel-node micrometastases (IBCSG 23-01): a phase 3 randomised controlled trial. Lancet Oncol 2013;14(4):297-305.

3. Giuliano AE, Ballman K, McCall L, Beitsch P, Whitworth PW, Blumencranz P, Leitch AM, Saha S, Morrow M, Hunt KK. Locoregional recurrence after sentinel lymph node dissection with or without axillary dissection in patients with sentinel lymph node metastases: long-term follow-up from the american college of surgeons oncology group (Alliance) ACOSOG Zoon Randomized Trial. Ann Surg 2016;264(3):413-20.

4. Giuliano AE, Hunt KK, Ballman KV, Beitsch PD, Whitworth PW, Blumencranz PW, Leitch AM, Saha S, McCall LM, Morrow M. Axillary dissection vs no axillary dissection in women with invasive breast cancer and sentinel node metastasis: a randomized clinical trial. JAMA 2011;305(6):569-75.

5. van Nijnatten TJ, Schipper RJ, Lobbes MB, Nelemans PJ, Beets-Tan RG, Smidt ML. The diagnostic performance of sentinel lymph node biopsy in pathologically confirmed node positive breast cancer patients after neoadjuvant systemic therapy: a systematic review and meta-analysis. Eur J Surg Oncol 2015;41(10):1278-87.

6. Mougalian SS, Hernandez M, Lei X, Lynch S, Kuerer HM, Symmans WF, Theriault RL, Fornage BD, Hsu L, Buchholz TA, Sahin AA, Hunt KK, Yang WT, Hortobagyi GN, Valero V. Ten-year outcomes of patients with breast cancer with cytologically confirmed axillary lymph node metastases and pathologic complete response after primary systemic chemotherapy. JAMA Oncol 2016;2(4):508-16.

7. Hennessy BT, Hortobagyi GN, Rouzier R, Kuerer H, Sneige N, Buzdar AU, Kau SW, Fornage B, Sahin A, Broglio K, Singletary SE, Valero V. Outcome after pathologic complete eradication of cytologically proven breast cancer axillary node metastases following primary chemotherapy. J Clin Oncol 2005;23(36):9304-11.

8. Meric F, Mirza NQ, Buzdar AU, Hunt KK, Ames FC, Ross MI, Pollock RE, Newman LA, Feig BW, Strom EA, Buchholz TA, McNeese MD, Hortobagyi GN, Singletary SE. Prognostic implications of pathological lymph node status after preoperative chemotherapy for operable T3NoMo breast cancer. Ann Surg Oncol 2000;7(6):435-40.

9. $\mathrm{CBO}$ Kwaliteitsinstituut voor de Gezondheidszorg. Vereniging van Integrale Kankercentra. Guideline 'Treatment of breast cancer' (Richtlijn 'Behandeling van het Mammacarcinoom') 2005.

10. Gradishar WJ, Anderson BO, Balassanian R, Blair SL, Burstein HJ, Cyr A, Elias AD, Farrar WB, Forero A, Giordano SH, Goetz M, Goldstein LJ, Hudis CA, Isakoff SJ, Marcom PK, Mayer IA, McCormick B, Moran M, Patel SA, Pierce LJ, Reed EC, Salerno KE, Schwartzberg LS, Smith KL, Smith ML, Soliman H, Somlo G, Telli M, Ward JH, Shead DA, Kumar R. Invasive breast cancer version 1.2016, NCCN clinical practice guidelines in oncology. J Natl Compr Canc Netw 2016;14(3):324-54.

11. NABON (2012) National breast cancer guideline, Oncoline. https://www.oncoline.nl/breastcancer. Accessed 23 Dec 2016

12. Caudle AS, Yang WT, Krishnamurthy S, Mittendorf EA, Black DM, Gilcrease MZ, Bedrosian I, Hobbs BP, DeSnyder SM, Hwang RF, Adrada BE, Shaitelman SF, Chavez-MacGregor M, Smith BD, Candelaria RP, Babiera GV, Dogan BE, Santiago L, Hunt KK, Kuerer HM. Improved axillary evaluation following neoadjuvant therapy for patients with node-positive breast cancer using selective evaluation of clipped nodes: implementation of targeted axillary dissection. J Clin Oncol 2016;34(10):1072-8. 
13. Donker M, Straver ME, Wesseling J, Loo CE, Schot M, Drukker CA, van Tinteren H, Sonke GS, Rutgers EJ, Vrancken Peeters MJ. Marking axillary lymph nodes with radioactive iodine seeds for axillary staging after neoadjuvant systemic treatment in breast cancer patients: the MARI procedure. Ann Surg 2015;261(2):378-82.

14. van der Ploeg IM, Nieweg OE, van Rijk MC, Valdes Olmos RA, Kroon BB. Axillary recurrence after a tumour-negative sentinel node biopsy in breast cancer patients: a systematic review and meta-analysis of the literature. Eur J Surg Oncol 2008;34(12):1277-84.

15. Boileau JF, Poirier B, Basik M, Holloway CM, Gaboury L, Sideris L, Meterissian S, Arnaout A, Brackstone M, McCready DR, Karp SE, Trop I, Lisbona A, Wright FC, Younan RJ, Provencher L, Patocskai E, Omeroglu A, Robidoux A. Sentinel node biopsy after neoadjuvant chemotherapy in biopsyproven node-positive breast cancer: the SN FNAC study. J Clin Oncol 2015;33(3):258-64. 



\section{Chapter}

\section{Does the TNM classification of solitary internal mammary lymph node metastases in breast cancer still apply?}

V Habraken*, TJA van Nijnatten*, L de Munck, M Moossdorff, EM Heuts, MBI Lobbes, ML Smidt

* Contributed equally to this work Breast Cancer Res Treat 2017;161:483-489 


\section{Abstract}

\section{Purpose}

TNM classification of solitary internal mammary lymph node metastases (IMLNMs) in breast cancer varies depending on their method of detection: sentinel lymph node biopsy ( $\mathrm{pN} 1 \mathrm{~b}$ ) or clinical examination including radiological and/or physical examination ( $\mathrm{pN} 2 \mathrm{~b}$ ). This study aimed to evaluate whether there is a difference in prognosis between both groups.

\section{Methods}

Data of all patients diagnosed with primary invasive epithelial breast cancer between 2005 and 2008 were obtained from the Netherlands Cancer Registry. Patients with IMLNMs were divided in groups according to their $\mathrm{pN}_{1} \mathrm{~b}$ and $\mathrm{pN} 2 \mathrm{~b}$ status. The main outcome measures disease-free survival (DFS) after 5 years and overall survival (OS) after 8 years were analyzed using Kaplan-Meier survival analysis. Cox regression analysis was used to determine independent predictors for DFS and OS.

\section{Results}

A total of 73 patients with $\mathrm{pN} 1 \mathrm{~b}$ status and 28 patients with $\mathrm{pN} 2 \mathrm{~b}$ status were included. DFS rate was $74.1 \%$ in the $\mathrm{pN} 1$ b group compared to $85.0 \%$ in the $\mathrm{pN} 2 \mathrm{~b}$ group $(P=0.211)$. Regarding OS, $20.5 \%(\mathrm{pN} 1 \mathrm{~b})$ and $25.0 \%(\mathrm{pN} 2 \mathrm{~b})$ of the patients deceased within 8 years of follow-up $(P=0.589)$. In multivariable cox regression analysis, nodal status was not statistically significant for DFS (HR 0.29 (95\% CI 0.04-2.33), $P=0.244$ ) or OS (HR 1.04 (95\% CI 0.37-2.89), $P=0.947$ ).

\section{Conclusions}

Although the TNM classification considers $\mathrm{pN}_{1} \mathrm{~b}$ and $\mathrm{pN}_{2} \mathrm{~b}$ to be distinct prognostic entities, we did not observe any prognostic differences between these groups. Therefore, solitary IMLNMs may be regarded as a single category in the future and revision of TNM classification should be considered. 


\section{Introduction}

In breast cancer staging, TNM classification is used to determine the anatomic extent of the disease and consequently identify specific subgroups with different prognoses. $^{1,2}$ Pathologic nodal staging is an important element in this classification as the presence of regional nodal metastases is associated with impaired survival. ${ }^{3}$ These metastases can occur not only in axillary but also in extra-axillary lymph nodes, such as intramammary, periclavicular, interpectoral, and internal mammary lymph nodes.

Pathological nodal staging of internal mammary lymph node metastases (IMLNMs) has changed over time. In the fourth (1987) and fifth edition (1997) of TNM classification, all IMLNMs were classified as $\mathrm{pN}_{3}$, because by that time IMLNMs were considered of great importance in formulating the prognosis of patients. ${ }^{4,5}$ Since the introduction of the sixth edition in 2002, IMLNMs are divided into $\mathrm{pN}_{1} \mathrm{~b}, \mathrm{pN}_{1}$, $\mathrm{pN}_{2} \mathrm{~b}$, or $\mathrm{pN}_{3} \mathrm{~b}$ status depending on their method of detection and possible concurrent axillary lymph node metastases. ${ }^{6,7}$ IMLNMs may be detected by physical and/or radiological examination or by sentinel lymph node biopsy (SLNB). ${ }^{8}$ Nowadays, solitary IMLNMs, in the absence of axillary lymph node metastases, are considered $\mathrm{pN} 1 \mathrm{~b}$ when detected at SLNB and pN2b when detected at clinical examination (including physical and/or radiological examination). ${ }^{7,9,10}$

Dividing solitary IMLNMs based on the method of detection, TNM implies a difference in prognosis between both groups. Therefore, the aim of this study was to evaluate whether a true difference in prognosis exists between $\mathrm{pN}_{1} \mathrm{~b}$ and $\mathrm{pN} 2 \mathrm{~b}$ status.

\section{Methods}

\section{Data collection}

Data of all patients diagnosed between 2005 and 2008 with primary invasive epithelial breast cancer were obtained from the Netherlands Cancer Registry (NCR), which is managed by the Netherlands Comprehensive Cancer Organisation (IKNL). The NCR ensures a high-quality data collection using specially trained employees who extract patient, tumour, and treatment characteristics directly from the patient records. Groups were defined according to $\mathrm{pNib}$ (IMLNMs detected at SLNB) and pN2b (IMLNMs detected at clinical examination) nodal status. Characteristics collected were age, tumour characteristics (size, location, stage, grade, subtype, and receptor status), and 
treatment characteristics (adjuvant chemotherapy, targeted therapy, endocrine therapy, and radiation therapy).

\section{Treatment}

During the study period, the Dutch national guideline of 2005 was in use. ${ }^{11}$ This guideline recommended regional treatment depending on nodal status: SLNB was indicated in clinically node-negative patients, based on physical examination, with axillary ultrasound being commonly used but not mandatory at that time. Clinically node-positive $\left(\mathrm{N}_{+}\right)$patients, patients with positive SLNB, or patients with a contraindication for SLNB underwent axillary lymph node dissection (ALND).

In all patients who underwent lumpectomy, whole-breast irradiation was indicated. After mastectomy, chest wall irradiation was indicated in the case of irradical resection, $\mathrm{pT}_{4}$ tumours, and involvement of the pectoral muscle or skin. For $\mathrm{pT}_{3}$ tumours, chest wall irradiation was considered individually. Irradiation of regional nodal fields was included in case of four or more axillary lymph node metastases or involvement of top axillary lymph nodes after ALND. The recommended dose was $45^{-50}$ Gy in 5 weeks, with a boost to 60-70 Gy when residual tumour was present.

Chemotherapy was recommended in all premenopausal $\mathrm{N}+$ women and in postmenopausal $\mathrm{N}+$ women aged 50-69 with estrogen receptor (ER)- and progesterone receptor (PR)-negative tumours. Furthermore, chemotherapy was considered in physically fit postmenopausal $\mathrm{N}+$ women aged 50-59 with ER- and PR-positive tumours and in $\mathrm{N}+$ women aged 6o-69 if four or more regional lymph nodes were involved. Chemotherapy regimen consisted of five courses of 5-Fluorouracil, Epirubicin, Cyclophosphamide (FEC) or six courses of Taxotere, Adriamycin, and Cyclophosphamide (TAC). Targeted therapy (trastuzumab) was recommended in selected cases in addition to chemotherapy in case of human epidermal growth factor 2 receptor amplification (HER2+). Endocrine therapy was recommended for all ER- and/or PR-positive tumours.

\section{Statistical analysis}

All analyses were performed using IBM SPSS Statistics version 23.0 (IBM Corporation, Armonk, New York, USA) and $P$ values <0.05 were considered statistically significant. Differences between $\mathrm{pN} 1 \mathrm{~b}$ and $\mathrm{pN} 2 \mathrm{~b}$ groups with regard to patient, tumour, and treatment characteristics were tested using the Fisher's Exact Test and Pearson Chi-square test for categorical variables and Mann-Whitney U test for continuous variables.

The main outcome measures were disease-free survival (DFS) after 5 years and overall survival (OS) after 8 years. DFS was defined as the absence of any first 
local, regional, or contralateral recurrence, distant metastasis, or mortality within 5 years. DFS rate included all patients without any event, who visited the hospital in the fifth year after diagnosis for regular check-up. OS was defined as the time interval between date of diagnosis and date of death or date of emigration, as obtained from the Municipal Personal Records Database and completed until December 31, 2014. Patients were censored at the date of their first event, date of last follow-up, date of death, or date of emigration, whatever came first. Patients without follow-up data were excluded from DFS analysis. DFS and OS were analyzed using Kaplan-Meier survival analysis and compared with the log-rank test.

Univariable and multivariable Cox regression analyses were used to determine relevant predictors for DFS and OS. Outcome measure was hazard ratio (HR) with corresponding $95 \%$ confidence intervals (CI). Due to the limited number of events, multivariable cox regression could only be performed with a limited number of variables. ${ }^{12}$ Nodal status together with the most significant variables in univariable cox regression was selected for multivariable cox regression.

\section{Results}

\section{General characteristics}

Between 2005 and 2008, a total of 51,239 patients were diagnosed with primary invasive epithelial breast cancer. After selection for $\mathrm{pN} 1 \mathrm{~b}(\mathrm{n}=73,72.3 \%)$ and $\mathrm{pN} 2 \mathrm{~b}$ $(\mathrm{n}=28,27.7 \%)$ status, a total of 101 patients remained, comprising $0.2 \%$ of the total population (Figure 9.1).

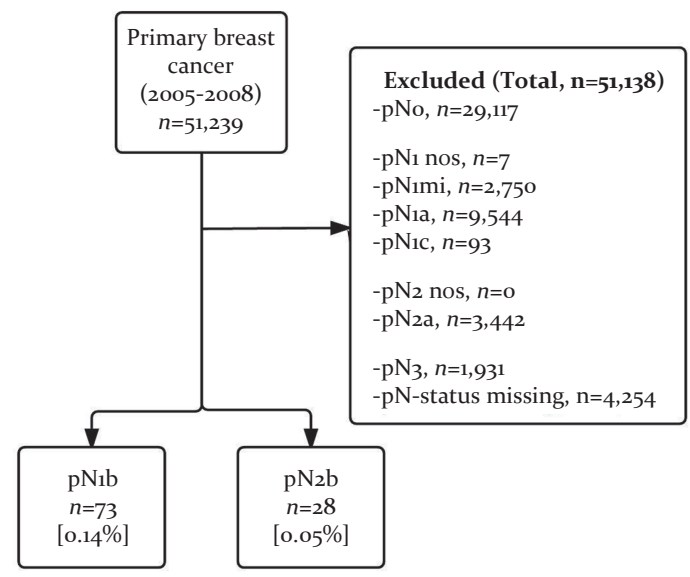

Figure 9.1 Flowchart of patient selection. Abbreviations: nos not otherwise specified, mi micrometastases, $p N 3$ includes $\mathrm{pN}_{3} \mathrm{a}, \mathrm{pN}_{3} \mathrm{~b}$, and $\mathrm{pN}_{3} \mathrm{c}$. 
In comparison to $\mathrm{pN} 1 \mathrm{~b}$ status, $\mathrm{pN} 2 \mathrm{~b}$ was associated with lower rates of $\mathrm{pTo}-1$ stage carcinoma (32 vs. $59 \%, P=0.016$ ), lower rates of grade $1-2$ carcinoma (32 vs. 63\%, $P=0.005)$, and larger mean tumour size (28 vs. $20 \mathrm{~mm}, P=0.008)$. A detailed overview of baseline patient, tumour, and treatment characteristics is shown in Table 9.1.

Table 9.1 Patient demographics and characteristics of tumour and treatment subdivided according to pN1b and $\mathrm{pN} 2 \mathrm{~b}$ status.

\begin{tabular}{|c|c|c|c|}
\hline & $\begin{array}{c}\mathrm{pNib} \\
(\mathrm{n}=73)\end{array}$ & $\begin{array}{c}\mathrm{pN}_{2} \mathrm{~b} \\
(\mathrm{n}=\mathbf{2 8})\end{array}$ & P-value \\
\hline Mean age, years (SD) & $55(14)$ & $58(17)$ & 0.693 \\
\hline Mean tumour size, mm (SD) & $20(11)$ & $28(15)$ & 0.008 \\
\hline \multicolumn{4}{|l|}{ pT-stage, n (\%) } \\
\hline To-1 & $43(59)$ & $9(39)$ & 0.016 \\
\hline $\mathrm{T} 2-4$ & $30(41)$ & $14(61)$ & 0.419 \\
\hline Unknown & & 5 & \\
\hline \multicolumn{4}{|l|}{ Tumour type, n (\%) } \\
\hline Ductal & $54(74)$ & $19(68)$ & 0.539 \\
\hline Lobular & $7(10)$ & $4(14)$ & 0.492 \\
\hline Mixed ductal \& lobular & $4(5)$ & $3(11)$ & 0.393 \\
\hline Other & $8(11)$ & $2(7)$ & 0.722 \\
\hline \multicolumn{4}{|l|}{ Grade, n (\%) } \\
\hline $1-2$ & $46(67)$ & $9(38)$ & 0.005 \\
\hline 3 & $23(33)$ & $15(62)$ & 0.040 \\
\hline Unknown & 4 & 4 & ${ }^{\circ}$ \\
\hline \multicolumn{4}{|l|}{ Receptor Status, n (\%) } \\
\hline $\mathrm{ER}+, \mathrm{PR}+, \mathrm{HER} 2-$ & $39(56)$ & $16(63)$ & 0.737 \\
\hline ER+, PR-, HER2- & $8(11)$ & $3(11)$ & 1.000 \\
\hline $\mathrm{ER}+, \mathrm{HER} 2+$ & $9(13)$ & $3(11)$ & 1.000 \\
\hline ER-, HER2+ & $3(4)$ & $3(11)$ & 0.344 \\
\hline Triple negative & $11(16)$ & $1(4)$ & 0.171 \\
\hline Unknown & 3 & 2 & 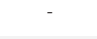 \\
\hline Chemotherapy, n (\%) & $44(60)$ & $17(61)$ & 0.968 \\
\hline Radiation therapy, n (\%) & $55(75)$ & $19(68)$ & 0.447 \\
\hline Trastuzumab, n (\%) & $13(18)$ & $3(11)$ & 0.546 \\
\hline Endocrine therapy, n (\%) & $51(70)$ & $19(68)$ & 0.845 \\
\hline
\end{tabular}

Abbreviations: SD Standard Deviation, $p T$-stage pathologic tumour stage, ER Estrogen Receptor, $P R$ Progesterone Receptor, HER2 Human epidermal growth factor receptor 2.

\section{Disease-free survival (DFS)}

Five-year follow-up data were complete for 54 patients $(74.0 \%)$ in the pNib group and 20 patients $(71.4 \%)$ in the pN2b group. An event occurred in 13 patients $(24.1 \%)$ in the $\mathrm{pN} 1 \mathrm{~b}$ group compared to two patients (10.0\%) in the $\mathrm{pN} 2 \mathrm{~b}$ group $(P=0.211)$ (Figure 9.2A). DFS rate was $74.1 \%$ in the $\mathrm{pNib}$ group and $85.0 \%$ in the $\mathrm{pN} 2 \mathrm{~b}$ group. When taking the effect of endocrine therapy and triple-negative subtype into account in multivariable Cox regression analysis, $\mathrm{pN}_{2} \mathrm{~b}$ status was 
not significantly different compared to pNib status (HR o.29 [95\% CI 0.04-2.33], $P=0.244)$. Neither endocrine therapy nor triple-negative subtype was identified as an independent predictor for improved DFS (HR 0.46 [95\% CI 0.12-1.86], $P=0.277$ and HR 1.56 [95\% CI 0.35-7.06], $P=0.561$, respectively) (Table 9.2).

A

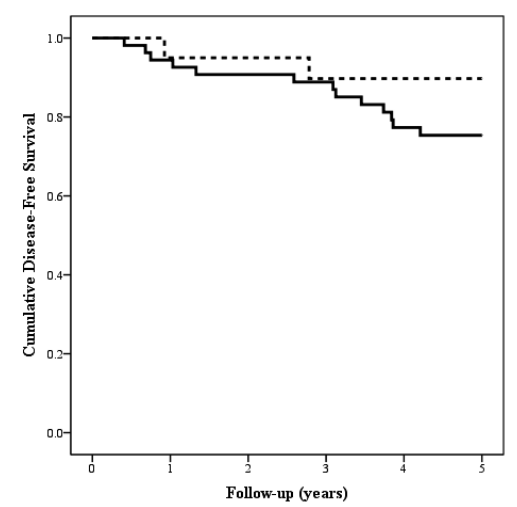

\begin{tabular}{|l|l|l|l|l|l|l|}
\hline pN1b & 54 & 51 & 48 & 47 & 40 & 24 \\
\hline pN2b & 20 & 19 & 18 & 17 & 17 & 11 \\
\hline
\end{tabular}

B

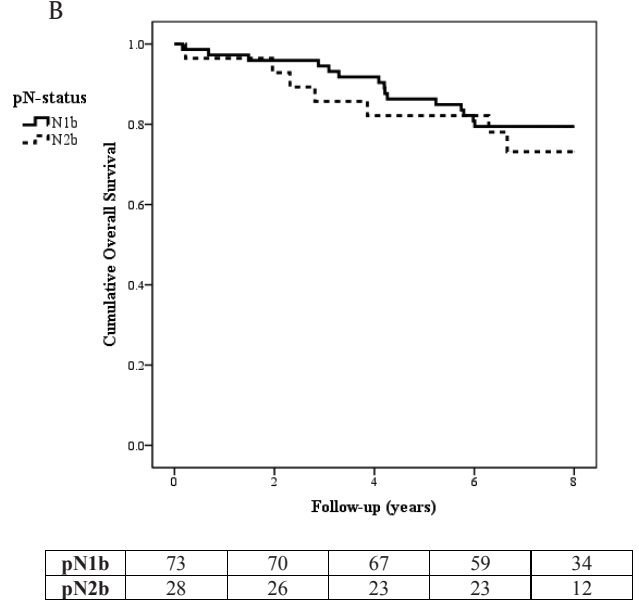

Figure 9.2 Kaplan-Meier survival curves of disease-free survival (A) and overall survival (B).

Table 9.2 Univariable and multivariable Cox Regression Analysis for Disease Free Survival.

\begin{tabular}{|c|c|c|c|c|}
\hline & \multicolumn{2}{|c|}{ Univariable Cox Regression } & \multicolumn{2}{|c|}{ Multivariable Cox Regression } \\
\hline & $\mathrm{HR}[95 \% \mathrm{CI}]$ & P-value & $\mathrm{HR}[95 \% \mathrm{CI}]$ & P-value \\
\hline $\mathrm{pN} 1 \mathrm{~b}$ & Reference & & Reference & \\
\hline $\mathrm{pN} 2 \mathrm{~b}$ & $0.40[0.09-1.77]$ & 0.227 & $0.29[0.04-2.33]$ & 0.244 \\
\hline $\begin{array}{l}\text { Tumour size } \\
\text { (Per mm increment) }\end{array}$ & $1.04[1.00-1.07]$ & 0.051 & & \\
\hline $\begin{array}{l}\text { pT-stage } \\
\text { T2-4 vs To-1 }\end{array}$ & $1.96[0.71-5.42]$ & 0.194 & & \\
\hline Tumour grade & & & & \\
\hline 3 vs $1-2$ & $1.07[0.37-3.09]$ & 0.897 & & \\
\hline $\begin{array}{l}\text { Triple negative subtype } \\
\text { Yes vs No }\end{array}$ & $3.5^{8}[1.10-11.63]$ & 0.034 & $1.56[0.35-7.06]$ & 0.561 \\
\hline $\begin{array}{l}\text { Radiation therapy } \\
\text { Yes vs No }\end{array}$ & $1.13[0.36-3.54]$ & 0.838 & & \\
\hline $\begin{array}{l}\text { Chemotherapy } \\
\text { Yes vs No }\end{array}$ & $1.21[0.44-3.35]$ & 0.709 & & \\
\hline $\begin{array}{l}\text { Endocrine therapy } \\
\text { Yes vs No }\end{array}$ & $0.25[0.09-0.70]$ & 0.008 & $0.46[0.12-1.86]$ & 0.277 \\
\hline $\begin{array}{l}\text { Trastuzumab } \\
\text { Yes vs No } \\
\end{array}$ & $0.33[0.04-2.47]$ & 0.200 & & \\
\hline
\end{tabular}

Abbreviations: HR Hazard Ratio, CI Confidence Interval, pT-stage pathological tumour stage. 


\section{Overall survival (OS)}

Median follow-up time of patients was 7.7 years (range 59 days-9.9 years). After 8 years of follow-up, 15 patients $(20.5 \%)$ in the pNib group and seven patients $(25.0 \%)$ in the $\mathrm{pN} 2 \mathrm{~b}$ group were deceased $(P=0.589)$ (Figure 9.2B). When taking the effect of tumour size (per $\mathrm{mm}$ increment), endocrine therapy, and trastuzumab into account in multivariable Cox regression analysis, pN2b status still was not significantly different compared to pNib status (HR 1.04 [95\% CI 0.37-2.89], $P=0.947$ ). Tumour size (HR 1.02 [95\% CI 1.00-1.05], $P=0.117$ ), endocrine therapy (HR 0.40 [95\% CI 0.15-1.04], $P=0.060$ ), and trastuzumab (HR 0.26 [95\% CI 0.04-1.98], $P=0.192$ ) did not have a statistically significant influence on OS (Table 9.3).

Table 9.3 Univariable and multivariable Cox Regression Analysis for Overall Survival.

\begin{tabular}{|c|c|c|c|c|}
\hline & \multicolumn{2}{|c|}{ Univariable Cox Regression } & \multicolumn{2}{|c|}{ Multivariable Cox Regression } \\
\hline & $\mathrm{HR}[95 \% \mathrm{CI}]$ & P-value & $\mathrm{HR}[95 \% \mathrm{CI}]$ & P-value \\
\hline $\mathrm{pNib}$ & Reference & & Reference & \\
\hline $\mathrm{pN}_{2} \mathrm{~b}$ & $1.28[0.52-3.14]$ & 0.590 & $1.04[0.37-2.89]$ & 0.947 \\
\hline \multicolumn{5}{|l|}{ Tumour size } \\
\hline (Per mm increment) & $1.04[1.01-1.06]$ & 0.003 & $1.02[1.00-1.05]$ & 0.117 \\
\hline \multicolumn{5}{|l|}{ pT-stage* } \\
\hline T2-4 vs To-1 & $2.19[0.91-5.29]$ & 0.082 & & \\
\hline \multicolumn{5}{|l|}{ Tumour grade } \\
\hline 3 vs $1-2$ & $1.73[0.67-4.49]$ & 0.259 & & \\
\hline \multicolumn{5}{|l|}{ Triple negative subtype } \\
\hline Yes vs No & $2.22[0.74-6.71]$ & 0.156 & & \\
\hline \multicolumn{5}{|l|}{ Radiation therapy } \\
\hline Yes vs No & $0.85[0.31-2.30]$ & 0.748 & & \\
\hline \multicolumn{5}{|l|}{ Chemotherapy } \\
\hline Yes vs No & $1.06[0.45-2.48]$ & 0.897 & & \\
\hline \multicolumn{5}{|l|}{ Endocrine therapy } \\
\hline Yes vs No & $0.30[0.13-0.69]$ & 0.005 & $0.40[0.15-1.04]$ & 0.060 \\
\hline \multicolumn{5}{|l|}{ Trastuzumab } \\
\hline Yes vs No & $0.22[0.03-1.63]$ & 0.138 & $0.26[0.04-1.98]$ & 0.192 \\
\hline
\end{tabular}

Abbreviations: HR Hazard Ratio, CI Confidence Interval, pT-stage pathological tumour stage. * Excluded from multivariable analysis due to collinearity with tumour size.

\section{Discussion}

According to the current TNM classification, patients with solitary IMLNMs are considered $\mathrm{pN}_{1} \mathrm{~b}$ when detected during SLNB and $\mathrm{pN}_{2} \mathrm{~b}$ when observed during clinical examination (including radiologic and/or physical examination), suggesting a prognostic difference between these two groups. ${ }^{1,2,6}$ However, our study demonstrated that both DFS after 5 years $(P=0.211)$ and OS after 8 years 
( $P=0.589)$ were not significantly different between both groups. Consequently, it is questionable whether the current TNM classification of IMLNMs is still appropriate.

The comparable prognosis of the $\mathrm{pN}_{1} \mathrm{~b}$ and $\mathrm{pN}_{2} \mathrm{~b}$ group in our study can be explained by the great improvements in imaging modalities over the last decade. In the past, clinical detection of IMLNMs was mostly restricted to large internal mammary lymph nodes found during physical examination (and later additional ultrasound if indicated).

Consequently, IMLNMs detected during physical examination were much larger and thus associated with worse prognosis than IMLNMs detected during SLNB. In distant past, 10-year overall survival ranged from o to $61 \%$ in patients with IMLNMs compared to our cohort of patients with SLNB-detected IMLNMs, of which only $20.5 \%$ of the patients deceased after 8 years of follow-up. ${ }^{6,13,14}$ Possible explanations for improved overall survival can be the introduction of other systemic regimen, such as trastuzumab, or detecting smaller IMLNMs with SLNB. Nowadays, the size of internal mammary lymph nodes detected using state-of-the-art imaging techniques such as PET/CT and MRI approaches the size of internal mammary nodes visualized during SLNB ${ }^{15-17}$ This suggests comparable prognosis of SLNB-detected IMLNMs and imaging-detected IMLNMs.

Routine evaluation of IMLNMs is controversial and is currently not recommended. The overriding arguments against routine evaluation of IMLNMs include their low incidence, their very limited impact on prognosis and treatment strategy, and the fact that tissue sampling is rather complex and associated with a risk of morbidity. ${ }^{13,18,19}$ However, detection of IMLNMs during radiological examinations and SLNB will continue to occur and possibly even increase with improving accuracy of these techniques. Their unambiguous and accurate classification will remain important as the detection of IMLNMs may alter nonsurgical treatment in patients. ${ }^{20}$ Current guidelines advise internal mammary irradiation in all patients with histologically proven and/or PET-positive IMLNMs and in patients with $\mathrm{N}_{2}$ status additional radiotherapy of the periclavicular region and/or thoracic wall can be advised. ${ }^{9,10,21}$ A previous study by Heuts et al. demonstrated that adjuvant treatment plans were changed in only $3.4 \%(27 / 789)$ of the patients based on the presence of IMLNMs. ${ }^{20}$ If TNM classification would be adapted by including all isolated IMLNMs in one group, then additional radiation therapy, besides internal mammary irradiation, could be omitted in these patients.

A major strength of this retrospective study is the use of a large populationbased dataset from the Netherlands Cancer Registry providing patient, tumour, and treatment characteristics. However, as metastatic spread to the internal mammary lymph node chain is rare, a limited number of patients were only available per subgroup. ${ }^{22}$ Early surgical series showed internal mammary 
involvement in $9.1 \%$ of patients undergoing extended radical mastectomy. ${ }^{23}$ According to our study, solitary IMLNMs were reported in only $0.2 \%$ of the population suggesting that IMLNMs may currently be underdiagnosed. Firstly, routine evaluation of IMLNMs is not recommended. Secondly, according to literature, superficial tracer injection (intradermal or periareaolar) often used during SLNB yields a lower visualization rate of internal mammary sentinel lymph nodes compared to intraparenchymal tracer injection (peritumoural, intratumoural, or subtumoural). ${ }^{24}$ All in all, the results of this study should be interpreted in the context of this small study population.

Furthermore, the staging technique used to classify patients as pN2b in our cohort was unknown. As a consequence, there was no distinction in our cohort of pN2b patients detected by for instance physical examination, ultrasound, MRI, or PET-CT. Yet, a previous study of Jochelson et al. demonstrated a difference in the prevalence between several imaging techniques for detecting internal mammary adenopathy. ${ }^{17}$

Another study limitation may be the completeness of data. Nodal status was missing in over 4000 patients (10.3\%) of the overall population of patients diagnosed with breast cancer in the Netherlands between 2005 and 2008. However, subclassification of $\mathrm{pN}_{1}$ (into $\mathrm{pN} 1 \mathrm{mi}, \mathrm{pN} 1 \mathrm{a}, \mathrm{pN} 1 \mathrm{~b}$, and $\mathrm{pN} \mathrm{N}_{1}$ ) and $\mathrm{pN} 2$ (into $\mathrm{pN} 2 \mathrm{a}$ and $\mathrm{pN} 2 \mathrm{~b}$ ) status seems to be accurately registered as in the $\mathrm{pN}_{1}$ group only seven patients were classified as $\mathrm{pN}_{1}$ not otherwise specified and none in the pN2 group (Figure 9.1). Therefore, registration of nodal status in our cohort was performed adequately.

In conclusion, our study did not observe any difference in prognosis between $\mathrm{pN} 1 \mathrm{~b}$ and $\mathrm{pN} 2 \mathrm{~b}$ in terms of DFS and OS. Since coincidental detection of IMLNMs during SLNB and radiological examinations will continue to occur and possibly even increase with improving accuracy of these techniques, their unambiguous and accurate classification will remain important. Therefore, more research on $\mathrm{pN}_{1} \mathrm{~b}$ and $\mathrm{pN} 2 \mathrm{~b}$ is advised and revision of TNM classification is desirable as solitary IMLNMs may be regarded as a single category. 


\section{References}

1. Sobin LH. TNM: principles, history, and relation to other prognostic factors. Cancer 2001;91(8 Suppl):1589-92.

2. Webber C, Gospodarowicz M, Sobin LH, Wittekind C, Greene FL, Mason MD, Compton C, Brierley J, Groome PA. Improving the TNM classification: findings from a 10-year continuous literature review. Int J Cancer 2014;135(2):371-8.

3. National Cancer Institute. Surveillance, epidemiology, and end results. SEER Stat Fact Sheets: Female Breast Cancer. http://seer.cancer.gov/statfacts/html/breast.html. Accessed 15 Oct 2016

4. Sobin LH, Fleming ID. TNM classification of malignant tumors, fifth edition. Union Internationale Contre le Cancer (UICC) and the American Joint Committee on Cancer (AJCC). Cancer 1997;80:1803-4.

5. Veronesi U, Cascinelli N, Bufalino R et al. Risk of internal mammary lymph node metastases and its relevance on prognosis of breast cancer patients. Ann Surg 1983;198(6):681-4

6. Singletary SE, Greene FL, Breast Task F. Revision of breast cancer staging: the 6th edition of the TNM Classification. Semin Surg Oncol 2003;21(1):53-9.

7. Sobin LH (2009) TNM classification, 7th edn. Wiley, Chichester Giuliano AE, Dale PS, Turner RR, Morton DL, Evans SW, Krasne DL. Improved axillary staging of breast cancer with sentinel lymphadenectomy. Ann Surg 1995;222(3):394-9 (discussion 399-401).

9. $\quad \mathrm{NABON}$ (2012) National breast cancer guideline, Oncoline

10. Senkus E, Kyriakides S, Ohno S, Penault-Llorca F, Poortmans P, Rutgers E, Zackrisson S, Cardoso F, Committee EG. Primary breast cancer: ESMO Clinical Practice Guidelines for diagnosis, treatment and follow-up. Ann Oncol 2015;26(Suppl 5):v8-30.

11. CBO Kwaliteitsinstituut voor de Gezondheidszorg. Vereniging van Integrale Kankercentra. Guideline 'Treatment of breast cancer' (Richtlijn 'Behandeling van het Mammacarcinoom') 2005.

12. Vittinghoff E, McCulloch CE. Relaxing the rule of ten events per variable in logistic and Cox regression. Am J Epidemiol 2007;165(6):710-8.

13. Madsen EV, Aalders KC, van der Heiden-van der Loo M, Gobardhan PD, van Oort PM, van der Ent FW, Rutgers EJ, Valdes Olmes RA, Elias SG, van Dalen T. Prognostic significance of tumorpositive internal mammary sentinel lymph nodes in breast cancer: a multicenter cohort study. Ann Surg Oncol 2015;22(13):4254-62.

14. Klauber-DeMore N, Bevilacqua JL, Van Zee KJ, Borgen P, Cody HS 3rd. Comprehensive review of the management of internal mammary lymph node metastases in breast cancer. J Am Coll Surg 2001;193(5):547-55.

15. Cheon H, Kim HJ, Lee SW, Kim DH, Lee CH, Cho SH, Shin KM, Lee SM, Kim GC, Kim WH. Internal mammary node adenopathy on breast MRI and PET/CT for initial staging in patients with operable breast cancer: prevalence and associated factors. Breast Cancer Res Treat 2016;160(3):523-30.

16. Mack M, Chetlen A, Liao J. Incidental internal mammary lymph nodes visualized on screening breast MRI. AJR Am J Roentgenol 2015;205(1):209-14.

17. Jochelson MS, Lebron L, Jacobs SS, Zheng J, Moskowitz CS, Powell SN, Sacchini V, Ulaner GA, Morris EA, Dershaw DD. Detection of internal mammary adenopathy in patients with breast cancer by PET/CT and MRI. AJR Am J Roentgenol 2015;205(4):899-904.

18. Heuts EM, van der Ent FW, von Meyenfeldt MF, Voogd AC. Internal mammary lymph drainage and sentinel node biopsy in breast cancer-a study on 1008 patients. Eur J Surg Oncol 2009;35(3):252-7.

19. Chen RC, Lin NU, Golshan M, Harris JR, Bellon JR. Internal mammary nodes in breast cancer: diagnosis and implications for patient management-a systematic review. J Clin Oncol 2008; 26(30):4981-9.

20. Heuts EM, van der Ent FW, Hulsewe KW, von Meyenfeldt MF, Voogd AC. Results of tailored treatment for breast cancer patients with internal mammary lymph node metastases. Breast 2009;18(4):254-8. 
21. Gradishar WJ, Anderson BO, Balassanian R, Blair SL, Burstein HJ, Cyr A, Elias AD, Farrar WB, Forero A, Giordano SH, Goetz M, Goldstein LJ, Hudis CA, Isakoff SJ, Marcom PK, Mayer IA, McCormick B, Moran M, Patel SA, Pierce LJ, Reed EC, Salerno KE, Schwartzberg LS, Smith KL, Smith ML, Soliman H, Somlo G, Telli M, Ward JH, Shead DA, Kumar R. Invasive Breast Cancer Version 1.2016, NCCN Clinical Practice Guidelines in Oncology. J Natl Compr Canc Netw 2016; 14(3):324-54.

22. Estourgie SH, Nieweg OE, Olmos RA, Rutgers EJ, Kroon BB (2004) Lymphatic drainage patterns from the breast. Ann Surg 2004;239(2):232-7.

23. Veronesi U, Cascinelli N, Greco M, Bufalino R, Morabito A, Galluzzo D, Conti R, De Lellis R, Delle Donne V, Piotti $\mathrm{P}$ et al. Prognosis of breast cancer patients after mastectomy and dissection of internal mammary nodes. Ann Surg 1985;202(6):702-7.

24. Ahmed M, Purushotham AD, Horgan K, Klaase JM, Douek M. Meta-analysis of superficial versus deep injection of radioactive tracer and blue dye for lymphatic mapping and detection of sentinel lymph nodes in breast cancer. Br J Surg 2015;102(3):169-81. 


\section{Chapter $\mathbf{1 0}$}

Discussion and future perspectives 



\section{Discussion}

The aim of this thesis was to investigate the enigma of lymph node staging in breast cancer patients, by improving diagnostic accuracy of nodal staging in order to achieve a more patient-tailored treatment with minimal invasive therapy and reduced morbidity. The first part concerns invasive lymph node staging techniques after completion of neoadjuvant systemic therapy: sentinel lymph node biopsy (SLNB) and RISAS-procedure (Radioactive Iodine Seed localization in the Axilla in axillary node positive breast cancer combined with Sentinel node procedure following neoadjuvant chemotherapy). The second part describes noninvasive lymph node staging techniques (axillary ultrasound (US), standard T2-weighted MRI (T2W-MRI), gadofosveset-enhanced axillary MRI (GDF-MRI), PET/CT and dedicated axillary hybrid PET/MRI). In the final part results concerning classification of lymph node metastases (infraclavicular lymph node metastases, axillary lymph node metastases after neoadjuvant systemic therapy and solitary internal mammary lymph node metastases) are reported.

\section{Part I - Invasive lymph node staging}

In clinically node negative patients, SLNB is generally accepted as standard axillary diagnostic technique for lymph node staging, with previously reported false-negative rate (FNR) of $4 \%$ and negative predictive value (NPV) of $96 \% .^{1}$ In clinically node positive patients treated with neoadjuvant systemic therapy, there is currently many ongoing research in order to explore a less invasive technique as opposed to axillary lymph node dissection (ALND) to accurately identify axillary pCR to prevent patients of ALND morbidity. According to chapter 2, SLNB in clinically node positive patients treated with neoadjuvant systemic therapy is inaccurate for assessment of axillary pathologic complete response (pCR), with a pooled FNR of $15.1 \%$ and a NPV of $86 \%$ or less. ${ }^{2}$ In subgroup analyses, FNR significantly improved in case two or more sentinel nodes were removed compared to only one (FNR $10.4 \%$ versus $23.9 \%, P=0.026$ ). This might suggest SLNB can be used as less invasive diagnostic technique in clinical practice, as opposed to axillary lymph node dissection (ALND). However, according to the SENTINA-trial only $69 \%(156 / 226)$ of patients with residual axillary nodal disease had 2 or more sentinel nodes. ${ }^{3}$ Consequently, SLNB can be considered an inaccurate technique in these patients, since over $30 \%$ of patients with residual axillary nodal disease would be incorrectly diagnosed and consequently treated then. 
Another less invasive technique to determine axillary $\mathrm{pCR}$ in clinically node positive patients after neoadjuvant systemic therapy was first described by Donker et al: the MARI-procedure (Marking the Axillary lymph node with Radioactive Iodine seeds). ${ }^{4}$ The MARI-procedure reported a promising FNR of $7 \%$. Axillary pCR in the MARI node was identified in 30 patients. Five out of these 30 patients had residual tumour burden in the ALND and therefore NPV remained 83\%. In other words, 1 out of 6 patients with negative MARI nodes will still have residual lymph node metastases in other axillary lymph nodes. Therefore, MARI-procedure as a solitary post-neoadjuvant systemic therapy axillary staging procedure may not seem sufficiently accurate for the prediction of axillary pCR. Consequently, MARIprocedure followed by axillary radiotherapy was suggested, depending on its response on imaging. ${ }^{6}$ Yet, previous studies already showed significantly more comorbidity once any axillary surgery was followed by axillary radiotherapy, compared to axillary surgery alone. ${ }^{7}$ A minimal invasive technique which can accurately assess the pathological status of remaining lymph nodes following neoadjuvant systemic therapy, without requiring adjuvant axillary radiotherapy, is therefore preferred.

An alternative technique was introduced by Caudle et al.: Targeted Axillary lymph node Dissection (TAD) ${ }^{8}$ In contrast to the MARI-procedure, an iodine seed was placed in the histopathologically confirmed axillary lymph node metastasis after completion of neoadjuvant systemic therapy. Recently, first results of the TAD-procedure were described in a cohort of 208 patients, of whom 85 patients underwent TAD-procedure and completion ALND. They reported promising results of FNR $2 \%(1 / 50)$ and NPV $97 \%(35 / 36) .{ }^{9}$ Limitations are the sample size of patients with axillary pCR in this cohort $(\mathrm{n}=36)$ and absence multicenter design of the study.

In summary, there is currently no less invasive procedure in clinical practice to accurately identify axillary pCR. However, the combination of both SLNB and MARI-procedure has the potential to discover small (SLNB) as well as larger nodal metastases (MARI). It certainly has the potential to outperform both SLNB and MARI-procedure alone. In chapter 3, a trial report of a new Dutch clinical prospective multicenter trial is described in detail, in which both SLND and MARI will be combined as one procedure: RISAS-procedure. ${ }^{10}$ For the next two years, a total of 225 patients will be included in this Dutch multicenter trial, in order to investigate whether the RISAS-procedure can truly identify axillary pCR accurately as compared to ALND. 


\section{Part II - Non-invasive lymph node staging}

Current European guidelines recommend axillary ultrasound (US) for lymph node staging in every patient. ${ }^{11}$ However, the role of US has become questionable since the ACOSOG Zoon trial demonstrated that a completion ALND can be safely omitted in clinically node negative patients based on physical examination. ${ }^{12}$ Yet, US is relevant though in selecting clinically node positive patients, who have a worse prognosis when compared to (clinically node negative) SLNB-positive patients. ${ }^{13}$ Consequently, excluding advanced axillary nodal disease ( $\mathrm{pN2}-3$ ) rather than detecting any positive lymph node is becoming more important. Previous studies reported probabilities of $\mathrm{pN}_{2}-3$ given node negative axillary US findings ranging from $4-7 \%{ }^{14,15}$ However, in case of suspicious nodal axillary findings, it cannot differentiate between limited axillary nodal disease ( $\mathrm{pN}_{1}$ ) and $\mathrm{pN}_{2}-3 .{ }^{14}$

The role of standard T2-weighted (T2W) breast MRI for axillary lymph node staging was investigated in chapter 4. After node negative findings on T2W-MRI, with a complete field of view of the axillary region, there is no further need for axillary US to safely exclude $\mathrm{pN}_{2}-3 .{ }^{16}$ In case of node positive findings on T2W-MRI, it outperformed axillary US regarding the probability of $\mathrm{pN}_{2}-3$ given one to three suspicious nodes ( $29 \%$ on US versus $12-15 \%$ on MRI). Hence, standard T2W-MRI can be used to more accurately define lymph node status in clinically node positive patients.

In order to further optimize MRI for differentiating between node negative and positive findings, lymph node specific contrast agent (Gadofosveset) was suggested. Gadofosveset was formerly used as blood pool agent in MRangiography. ${ }^{17}$ It is characterized by its binding to serum albumin, which is also present in lymph nodes. Hypothetically, concentration of albumin decreases in case of cancer invasion into lymph nodes, which allows differentiation between benign (high uptake of contrast agent) and malignant (low uptake of contrast agent) lymph nodes. ${ }^{18}$ In rectal cancer, the use of gadofosveset-enhanced MRI (GDF-MRI) improved diagnostic performance for lymph node staging. ${ }^{19,20}$ In breast cancer nodes, a feasibility study demonstrated promising results with GDFMRI. ${ }^{21}$ Of special interest is the high NPV which even outperforms the NPV of SLNB (NPV 96\%) suggesting that axillary surgery could be safely omitted in clinically node negative patients. ${ }^{1}$ In chapter 5 , the diagnostic performance of GDF-MRI was investigated in a larger cohort of 97 patients. The diagnostic performance of GDF-MRI, on a node-by-node analysis, was: sensitivity $59 \%$, specificity $87 \%$, positive predictive value $65 \%$ and NPV $84 \%$. However, a learning curve existed and NPV improved in the expert GDF-reader from $79 \%$ to $92 \%$, after reading 176 lymph nodes (AUC 0.95). Therefore, GDF-MRI could potentially be used in clinical practice, but unfortunately the manufacturer recently halted 
production of gadofosveset. Consequently, our validation study was closed early and future use of the contrast-agent gadofosveset is insecure.

Besides MRI, Koolen et al. demonstrated that PET/CT can be considered of added value for lymph node staging in breast cancer with a positive predictive value of $98 \%$ for axillary lymph node metastases. ${ }^{22}$ Unfortunately, PET/CT suffered from a relatively low NPV of $53 \%$ in case of non-FDG avid lymph nodes. This indicates that the overall use of PET/CT is limited in clinical practice since axillary surgery in patients with negative findings on PET/CT cannot be safely

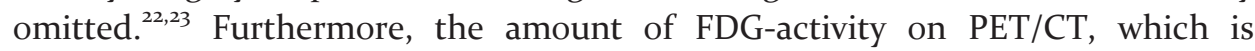
indicative to differentiate between benign and malignant findings, varies per subtypes. It is most accurate in estrogen positive $(\mathrm{ER}+)$ and Her2- or triple negative subtypes, in contrast to MRI which is most accurate in ER+/Her2+ and ER-/Her2+ subtypes. ${ }^{24,25}$

A combination of both promising imaging modalities, i.e. MRI and PET, would be a next step to further optimize non-invasive lymph node staging: the accuracy to identify patients with axillary lymph node metastases by PET (high PPV) and the accuracy to exclude patients without axillary lymph node metastases by MRI (high NPV). After having overcome the technical hurdles, the combination of both imaging modalities was successfully achieved in 2008 by introducing a hybrid PET/MR system. ${ }^{26}$ A previous study of Melsaether et al. reported an improved identification for axillary lymph node metastases with the use of PET/MRI compared to PET/CT, with an increased sensitivity from $88 \%$ to $100 \%{ }^{27}$ However, this study only differentiated between negative and positive axillary lymph nodes rather that reporting the number of suspicious nodes. In current clinical practice, the number of suspicious nodes is important regarding adjuvant axillary treatment (i.e. adjuvant radiation therapy of periclavicular region and thoracic wall). In chapter 6, a pilot study demonstrated improved differentiation between limited and advanced axillary nodal disease with the use of a dedicated axillary hybrid PET/MR protocol compared to current imaging modalities like ultrasound, MRI and PET/CT. According to dedicated axillary hybrid PET/MRI findings, clinical nodal status changed in up to $75 \%$ of patients. Therefore, it was concluded that dedicated axillary hybrid PET/MR could have potential value in clinically node positive patients to improve differentiation between limited and advanced axillary nodal disease. 


\section{Part III - Classification of lymph node staging}

The TNM classification system not only aims to provide an indication of prognosis, but also assists in treatment planning and evaluation of treatment effects. ${ }^{28}$ In breast cancer, correct classification of lymph nodes has major consequences since treatment strategies are based on TNM classification. According to this thesis, reclassification of both solitary internal mammary lymph node metastases ( $\left.\mathrm{pN}_{1} \mathrm{~b} / \mathrm{pN} 2 \mathrm{~b}\right)$ and infraclavicular lymph node metastases ( $\mathrm{pN}_{3} \mathrm{a}$ ) can be advised. Solitary internal mammary lymph node metastases should be considered as a single category in future. ${ }^{29}$ In addition, infraclavicular lymph node metastases, with a maximum of nine lymph node metastases in total, should be considered as $\mathrm{pN}_{2 \mathrm{a}}$ rather than $\mathrm{pN}_{3} \mathrm{a}^{30}$.

Furthermore, the impact of remaining nodal disease after completion of neoadjuvant systemic therapy was investigated. Our research showed that prognosis of axillary pCR and isolated tumor cells and/or micrometastases was comparable in clinically node positive patients treated with neoadjuvant systemic therapy followed by ALND. ${ }^{31}$ Therefore, small metastases seem less relevant compared to macrometastases. This supports the role of imaging after completion of neoadjuvant systemic therapy, since small metastases (i.e. isolated tumour cells/micrometastases) can be missed on imaging modalities without any prognostic consequences for the patient.

\section{Future perspectives}

Future studies should further explore the diagnostic performance of dedicated axillary hybrid PET/MRI for lymph node staging in breast cancer. Hypothetically, dedicated axillary hybrid PET/MRI could be used prior to surgery in any patient to evaluate potential presence of axillary lymph node metastases. The impact could be practice changing with better stratification of patients for axillary surgery. In addition, cost-effectiveness studies should be performed to investigate whether dedicated axillary hybrid PET/MRI can potentially serve as non-invasive lymph node staging tool to replace axillary surgery in clinically node negative patients. In clinically node positive patients, nodal response after neoadjuvant systemic therapy can potentially be assessed with dedicated axillary hybrid PET/MRI too. This allows clinically node positive patients who converted to axillary pCR after administration of neoadjuvant systemic therapy to omit axillary surgery and minimizing the risk of morbidity caused by surgery. 


\section{Conclusion and recommendations}

The ongoing RISAS trial will answer the question whether RISAS-procedure is an accurate method for less invasive axillary lymph node staging as opposed to ALND in clinically node positive patients treated with neoadjuvant chemotherapy with the aim to accurately identify axillary pCR. Eventually, RISAS-procedure could prevent morbidity, by omitting ALND in patients with negative RISAS nodes.

Meanwhile, non-invasive lymph node staging will be further optimized to improve evaluation of lymph nodes after neoadjuvant systemic therapy. Determination of these lymph nodes should focus on residual macrometastases rather than any extent of nodal disease.

In clinical practice, axillary US only is sufficient in case of node negative findings treated with SLNB. Yet, T2W-MRI covering the entire axillary region can replace axillary US if the MR shows node negativity.

In clinically node positive patients, $\mathrm{T}_{2} \mathrm{~W}$-MRI is more accurate compared to axillary US to define the number of suspicious axillary lymph nodes. Dedicated axillary hybrid PET/MRI has potential to improve accuracy of lymph node staging, especially regarding the number of suspicious lymph nodes. Solid evidence has yet to be built and together with its limited availability only in research institutes this makes that PET/MRI is not yet ready for clinical practice. 


\section{References}

1. Krag DN, Anderson SJ, Julian TB, Brown AM, Harlow SP, Ashikaga T, Weaver DL, Miller BJ, Jalovec LM, Frazier TG, Noyes RD, Robidoux A, Scarth HM, Mammolito DM, McCready DR, Mamounas EP, Costantino JP, Wolmark N, National Surgical Adjuvant B, Bowel P. Technical outcomes of sentinel-lymph-node resection and conventional axillary-lymph-node dissection in patients with clinically node-negative breast cancer: results from the NSABP B-32 randomised phase III trial. Lancet Oncol 2007;8(10):881-8.

2. van Nijnatten TJ, Schipper RJ, Lobbes MB, Nelemans PJ, Beets-Tan RG, Smidt ML. The diagnostic performance of sentinel lymph node biopsy in pathologically confirmed node positive breast cancer patients after neoadjuvant systemic therapy: A systematic review and meta-analysis. Eur J Surg Oncol 2015;41(10):1278-87.

3. Kuehn T, Bauerfeind I, Fehm T, Fleige B, Hausschild M, Helms G, Lebeau A, Liedtke C, von Minckwitz G, Nekljudova V, Schmatloch S, Schrenk P, Staebler A, Untch M. Sentinel-lymph-node biopsy in patients with breast cancer before and after neoadjuvant chemotherapy (SENTINA): a prospective, multicentre cohort study. Lancet Oncol 2013;14(7):609-18.

4. Donker M, Straver ME, Wesseling J, Loo CE, Schot M, Drukker CA, van Tinteren H, Sonke GS, Rutgers EJ, Vrancken Peeters MJ. Marking axillary lymph nodes with radioactive iodine seeds for axillary staging after neoadjuvant systemic treatment in breast cancer patients: the MARI procedure. Ann Surg 2015;261(2):378-82.

5. Vugts G, Nieuwenhuijzen GA, Maaskant-Braat AJ, Schipper RJ, Smidt ML. Axillary response monitoring after neoadjuvant chemotherapy in breast cancer: can we avoid the morbidity of axillary treatment? Ann Surg 2016;263(2):e28-9.

6. Van der Noordaa ME, van Duijnhoven FH, Vrancken-Peeters MT. Minder okselklierdissecties bij borstkankerpatiënten na neoadjuvante systemische therapie door gebruik van PET/CT en de MARI-procedure. NTvO 2017;14:55-61.

7. Ashikaga T, Krag DN, Land SR, Julian TB, Anderson SJ, Brown AM, Skelly JM, Harlow SP, Weaver DL, Mamounas EP, Costantino JP, Wolmark N, National Surgical Adjuvant Breast BP. Morbidity results from the NSABP B-32 trial comparing sentinel lymph node dissection versus axillary dissection. J Surg Oncol 2010;102(2):111-8.

8. Caudle AS, Yang WT, Mittendorf EA, Black DM, Hwang R, Hobbs B, Hunt KK, Krishnamurthy S, Kuerer HM. Selective surgical localization of axillary lymph nodes containing metastases in patients with breast cancer: a prospective feasibility trial. JAMA Surg 2015; 150(2):137-43.

9. Caudle AS, Yang WT, Krishnamurthy S, Mittendorf EA, Black DM, Gilcrease MZ, Bedrosian I, Hobbs BP, DeSnyder SM, Hwang RF, Adrada BE, Shaitelman SF, Chavez-MacGregor M, Smith BD, Candelaria RP, Babiera GV, Dogan BE, Santiago L, Hunt KK, Kuerer HM. Improved axillary evaluation following neoadjuvant therapy for patients with node-positive breast cancer using selective evaluation of clipped nodes: implementation of targeted axillary dissection. J Clin Oncol, 2016;34(10):1072-8.

10. van Nijnatten TJA, Simons JM, Smidt ML, van der Pol CC, van Diest PJ, Jager A, van Klaveren D, Kam BLR, Lobbes MBI, de Boer M, Verhoef K, Koppert LB, Luiten EJT. A novel less-invasive approach for axillary staging after neoadjuvant chemotherapy in patients with axillary nodepositive breast cancer by combining radioactive iodine seed localization in the axilla with the sentinel node procedure (RISAS): a Dutch prospective multicenter validation study. Clin Breast Cancer 2017.

11. Senkus E, Kyriakides S, Ohno S, Penault-Llorca F, Poortmans,P, Rutgers E, Zackrisson S, Cardoso F, Committee EG. Primary breast cancer: ESMO Clinical Practice Guidelines for diagnosis, treatment and follow-up. Ann Oncol 2015;26 Suppl 5:v8-30.

12. Giuliano AE, Hunt KK, Ballman KV, Beitsch PD, Whitworth PW, Blumencranz PW, Leitch AM, Saha S, McCall LM, Morrow M. Axillary dissection vs no axillary dissection in women with invasive breast cancer and sentinel node metastasis: a randomized clinical trial. JAMA 2011; 305(6):569-75. 
13. Verheuvel NC, van den Hoven I, Ooms HW, Voogd AC, Roumen RM. The role of ultrasoundguided lymph node biopsy in axillary staging of invasive breast cancer in the post-ACOSOG Zoo11 trial era. Ann Surg Oncol 2015;22(2):409-15.

14. Schipper RJ, van Roozendaal LM, de Vries B, Pijnappel RM, Beets-Tan RG, Lobbes MB, Smidt ML. Axillary ultrasound for preoperative nodal staging in breast cancer patients: is it of added value? Breast 2013;22(6):1108-13.

15. Neal CH, Daly CP, Nees AV, Helvie MA. Can preoperative axillary US help exclude $\mathrm{N}_{2}$ and $\mathrm{N}_{3}$ metastatic breast cancer? Radiology 2010;257(2):335-41.

16. van Nijnatten TJ, Ploumen EH, Schipper RJ, Goorts B, Andriessen EH, Vanwetswinkel S, Schavemaker M, Nelemans P, de Vries B, Beets-Tan RG, Smidt ML, Lobbes MB. Routine use of standard breast MRI compared to axillary ultrasound for differentiating between no, limited and advanced axillary nodal disease in newly diagnosed breast cancer patients. Eur J Radiol 2016; 85(12):2288-2294.

17. Perreault P, Edelman MA, Baum RA, Yucel EK, Weisskoff RM, Shamsi K, Mohler ER, 3rd. MR angiography with gadofosveset trisodium for peripheral vascular disease: phase II trial. Radiology 2003;229(3):811-20.

18. Lahaye MJ, Beets GL, Engelen SME, Voth M, Leiner T, Lambregts DMJ, Beets-Tan RGH. Gadovosfeset Trisodium (Vasovist) enhanced MR lymph node detection: initial observations. Open Magnetic Reson J 2009;2:1-5.

19. Lambregts DM, Beets GL, Maas M, Kessels AG, Bakers FC, Cappendijk VC, Engelen SM, Lahaye MJ, de Bruine AP, Lammering G, Leiner T, Verwoerd JL, Wildberger JE, Beets-Tan RG. Accuracy of gadofosveset-enhanced MRI for nodal staging and restaging in rectal cancer. Ann Surg 2011; 253(3):539-45.

20. Heijnen LA, Lambregts DM, Martens MH, Maas M, Bakers FC, Cappendijk VC, Oliveira P, Lammering G, Riedl RG, Beets GL, Beets-Tan RG. Performance of gadofosveset-enhanced MRI for staging rectal cancer nodes: can the initial promising results be reproduced? Eur Radiol 2014; 24(2):371-9

21. Schipper RJ, Smidt ML, van Roozendaal LM, Castro CJ, de Vries B, Heuts EM, Keymeulen KB, Wildberger JE, Lobbes MB, Beets-Tan RG. Noninvasive nodal staging in patients with breast cancer using gadofosveset-enhanced magnetic resonance imaging: a feasibility study. Invest Radiol 2013;48(3):134-9.

22. Koolen BB, Valdes Olmos RA, Elkhuizen PH, Vogel WV, Vrancken Peeters MJ, Rodenhuis S, Rutgers EJ. Locoregional lymph node involvement on 18F-FDG PET/CT in breast cancer patients scheduled for neoadjuvant chemotherapy. Breast Cancer Res Treat 2012;135(1):231-40.

23. Liang X, Yu J, Wen B, Xie J, Cai Q, Yang Q. MRI and FDG-PET/CT based assessment of axillary lymph node metastasis in early breast cancer: a meta-analysis. Clin Radiol 2017;72(4):295-301.

24. Koolen BB, Valdes Olmos RA, Wesseling J, Vogel WV, Vincent AD, Gilhuijs KG, Rodenhuis S, Rutgers EJ, Vrancken Peeters MJ. Early assessment of axillary response with (1)(8)F-FDG PET/CT during neoadjuvant chemotherapy in stage II-III breast cancer: implications for surgical management of the axilla. Ann Surg Oncol 2013;20(7):2227-35.

25. Grimm LJ, Johnson KS, Marcom PK, Baker JA, Soo MS. Can breast cancer molecular subtype help to select patients for preoperative MR imaging? Radiology 2015;274(2):352-8.

26. Pichler BJ, Judenhofer MS, Wehrl HF. PET/MRI hybrid imaging: devices and initial results. Eur Radiol 2008;18(6):1077-86.

27. Melsaether AN, Raad RA, Pujara AC, Ponzo FD, Pysarenko KM, Jhaveri K, Babb JS, Sigmund EE, Kim SG, Moy LA. Comparison of whole-body (18)F FDG PET/MR imaging and whole-body (18)F FDG PET/CT in terms of lesion detection and radiation dose in patients with breast cancer. Radiology 2016;281(1):193-202.

28. Webber C, Gospodarowicz M, Sobin LH, Wittekind C, Greene FL, Mason MD, Compton C, Brierley J, Groome PA. Improving the TNM classification: findings from a 10-year continuous literature review. Int J Cancer 2014;135(2):371-8.

29. Habraken V, van Nijnatten TJ, de Munck L, Moossdorff M, Heuts EM, Lobbes MB, Smidt ML. Does the TNM classification of solitary internal mammary lymph node metastases in breast cancer still apply? Breast Cancer Res Treat 2017;161(3):483-9. 
30. van Nijnatten TJA, Moossdorff M, de Munck L, Goorts B, Vane MLG, Keymeulen K, Beets-Tan RGH, Lobbes MBI, Smidt ML. TNM classification and the need for revision of pN3a breast cancer. Eur J Cancer 2017;79:23-30.

31. van Nijnatten TJ, Simons JM, Moossdorff M, de Munck L, Lobbes MB, van der Pol CC, Koppert LB, Luiten EJ, Smidt ML. Prognosis of residual axillary disease after neoadjuvant chemotherapy in clinically node-positive breast cancer patients: isolated tumor cells and micrometastases carry a better prognosis than macrometastases. Breast Cancer Res Treat 2017;163(1):159-66. 

Summary 



\section{Summary}

The aim of this thesis was to investigate the enigma of lymph node staging in breast cancer patients, by improving the diagnostic accuracy of nodal staging in order to achieve a more patient-tailored treatment with minimal invasive therapy and reduced morbidity.

\section{Part I - Invasive lymph node staging}

Currently, there is ongoing research exploring less invasive techniques as opposed to axillary lymph node dissection (ALND) to accurately identify axillary pathologic complete response $(\mathrm{pCR})$ in clinically node positive patients treated with neoadjuvant systemic therapy, in order to prevent patients of ALND related morbidity. Chapter 2 described a systematic review and meta-analysis of a total of eight studies investigating sentinel lymph node biopsy (SLNB) as less invasive technique as opposed to ALND. According to our results, SLNB is not able to accurately identify axillary pCR, with a pooled identification rate of $92.3 \%$, pooled false-negative rate of $15.1 \%$ and negative predictive values ranging from $61.5 \%$ $86.3 \%$.

Chapter 3 reported the rationale and design of a Dutch prospective multicenter validation study: RISAS (Radioactive Iodine Seed localization in the Axilla with the Sentinel node procedure). For the next two years, this study will investigate the diagnostic accuracy of the RISAS-procedure in 225 clinically node positive patients treated with neoadjuvant systemic therapy.

\section{Part II - Non-invasive lymph node staging}

In chapter 4, standard breast MRI for axillary lymph node staging was compared to dedicated axillary ultrasound. Two independent breast radiologists reassessed breast MRI exams of 377 patients. The probability of $\mathrm{pN}_{2}-3$ given cNo for breast MRI and axillary ultrasound was $0.7-0.9 \%$ versus $1.5 \%$. In case of $\mathrm{cN}_{1}$, probability of $\mathrm{pN2}-3$ on breast MRI was $11.6-15.4 \%$ versus $29.0 \%$ on axillary ultrasound. It was concluded that evaluation of axillary nodal status on standard breast MRI is comparable to dedicated axillary ultrasound.

A validation study on gadofosveset-enhanced axillary MRI for nodal (re)staging was described in chapter 5 . Ninety patients underwent $\mathrm{T}_{2}$ weightedand gadofosveset-enhanced axillary MRI. Two independent readers assessed these 
axillary MRI exams. After reading 176 nodes, sensitivity, specificity, PPV, NPV and AUC for reader 1 improved from $38 \%, 89 \%, 56 \%, 79 \%$ and 0.71 to $60 \%, 93 \%, 64 \%$, $92 \%$ and 0.95 . AUC of reader 2 improved from o.69 to 0.79 . Based on these results, gadofosveset-enhanced axillary MRI was considered to be a potential non-invasive imaging tool for nodal (re)staging in breast cancer.

A feasibility study investigating dedicated axillary 18F-FDG hybrid PET/MRI was reported in chapter 6 . Twelve clinically node positive patients underwent axillary ultrasound and dedicated axillary PET/MRI. Nine of these twelve patients also underwent whole-body PET/CT. Regarding the number of suspicious axillary lymph nodes, dedicated axillary hybrid PET/MRI changed nodal status when compared to standard imaging modalities: $40 \%$ compared to axillary ultrasound, $75 \%$ compared to T2 weighted MRI, $40 \%$ compared to contrast-enhanced MRI and $22 \%$ compared to PET/CT. Therefore, dedicated axillary $18 \mathrm{~F}-\mathrm{FDG}$ hybrid PET/MRI was considered feasible and might improve diagnostic performance of axillary nodal staging in clinically node positive patients.

\section{Part III - Classification of lymph node staging}

In chapter 7 , patients with a pN3a nodal status (i.e. at least one infraclavicular lymph node metastasis or $\geq$ ten axillary lymph node metastases) were investigated, using data from the Netherlands Cancer Registry. According to the results from a cohort of 1,788 patients with $\mathrm{pN}_{32}$, patients with $\geq$ ten axillary lymph node metastases had a significantly worse prognosis compared to patients with at least one infraclavicular lymph node metastasis and nine or less lymph node metastases in total (disease-free survival (DFS): $48.8 \%$ versus $63.8 \%$, $P=0.036$; overall survival (OS): $46.6 \%$ versus $63.9 \%, P=0.042)$. In addition, prognosis of patients with $\mathrm{pN}_{3}$ a based on at least one infraclavicular lymph node metastasis was comparable to patients with pN2a (four to nine axillary lymph node metastases) (DFS: $63.8 \%$ versus $67.3 \%, P=0.491$; OS: $63.9 \%$ versus $65.5 \%$, $P=0.233)$. Therefore, reclassification of infraclavicular lymph node metastases in the next TNM classification was suggested.

Chapter 8 compared prognosis of clinically node positive patients treated with neoadjuvant systemic therapy, subdivided by ypN status: axillary pCR (ypNo), residual axillary isolated tumour cells or micrometastases (ypNitc/mi) and residual axillary macrometastases (ypN1-3). In a cohort of 1,347 patients, obtained from data of the Netherlands Cancer Registry, similar prognosis was demonstrated between ypNo and ypNitc/mi (DFS: Hazard Ratio (HR) 1.38 (o.404.79), $P=0.613$; OS: HR 0.92 (o.27-3.09), $P=0.889$ ). Prognosis between ypNo and 
ypN1-3 was statistically significant different (DFS: HR 1.78 (1.06-3.0o), $P=0.031$; OS: HR 1.70 (1.07-2.71), $P=0.026)$. In conclusion, future studies should consider ypNo and ypNitc/mi as one entity.

Besides axillary lymph node metastases, internal mammary lymph node metastases can reduce prognosis as well. Solitary internal mammary lymph node metastases, in the absence of axillary lymph node metastases, are divided in the current TNM classification according to method of detection: at SLNB (pNib) or at clinical examination ( $\mathrm{pN} 2 \mathrm{~b}$ ). However, in chapter 9 no prognostic difference was observed between both subgroups in a cohort consisting of 101 patients with solitary internal mammary lymph node metastases, obtained from data of the Netherlands Cancer Registry, after multivariable cox regression analyses (DFS: HR 0.29 (o.04-2.33), $P=0.244$; OS: HR 1.04 (0.37-2.89), $P=0.947$ ). Therefore, both subgroups may be regarded as a single category. 

Samenvatting 



\section{Samenvatting}

Het doel van dit proefschrift was het onderzoeken van lymfeklierstadiëring bij patiënten met borstkanker, door het verbeteren van diagnostische accuratesse op het gebied van lymfeklierstadiëring. Zodoende kan een meer patiëntgerichte behandeling uitgevoerd worden met minimaal invasieve therapie en verminderde kans op morbiditeit.

\section{Deel I - Invasieve lymfeklierstadiëring}

Momenteel zijn er verschillende studies die minder invasieve technieken in vergelijking tot een okselklierdissectie onderzoeken in klinisch klier-positieve patiënten behandeld met neoadjuvante systemische therapie. Deze techniek dient axillaire pathologisch complete respons (pCR) accuraat te kunnen identificeren. Indien een accurate techniek is vastgesteld, kan morbiditeit van een okselklierdissectie bij patiënten met axillaire pCR worden vermeden. Hoofdstuk 2 onderzocht de schildwachtklierprocedure als minder invasieve techniek in vergelijking tot een okselklierdissectie bij klinisch klier-positieve patiënten behandeld met neoadjuvante systemische therapie, door middel van een systematisch review en meta-analyse van in totaal acht studies. Op basis van deze resultaten werd geconcludeerd dat de schildwachtklierprocedure geen accurate techniek is voor het identificeren van axillaire $\mathrm{pCR}$, met een gepoolde identificatiewaarde van $92.3 \%$, gepoolde fout-negatieve ratio van $15.1 \%$ en een negatief voorspellende waarde van $61.5 \%$ - $86.3 \%$.

In hoofdstuk 3 werd de achtergrond en methode van een Nederlandse prospectieve multicenter validatiestudie toegelicht: RISAS (lokalisatie van een axillair geplaatst radioactief jodiumzaadje in combinatie met de schildwachtklierprocedure). De komende twee jaar onderzoekt deze studie de diagnostische accuratesse van de RISAS-procedure in 225 klinisch klier-positieve patiënten behandeld met neoadjuvante systemische therapie.

\section{Deel II - Non-invasieve lymfeklierstadiëring}

Hoofdstuk 4 onderzocht de waarde van standaard MRI van de mammae voor axillaire lymfeklierstadiëring in vergelijking tot axillaire echografie. Twee onafhankelijke mamma-radiologen herbeoordeelden elk afzonderlijk 377 MRIscans. De kans op pN2-3 in geval van cNo volgens MRI mammae en axillaire echografie was $0.7-0.9 \%$ versus $1.5 \%$. In geval van $\mathrm{cN}_{1}$, was de kans op $\mathrm{pN}_{2-3}$ 
volgens MRI mammae was $11.6-15.4 \%$ versus $29.0 \%$ volgens axillaire echografie. Op basis van deze resultaten werd geconcludeerd dat evaluatie van axillaire lymfeklieren op MRI mammae vergelijkbaar is met echografie.

In hoofdstuk 5 werd de waarde van gadofosveset-versterkte axillaire MRI voor (her)stadiëring van lymfeklieren onderzocht. Negentig patiënten ondergingen $\mathrm{T}_{2}$ gewogen en gadofosveset-versterkte axillaire MRI-scans. Twee onafhankelijke radiologen beoordeelden al deze MRI-scans. Na beoordeling van 176 lymfeklieren door de eerste beoordelaar, verbeterden sensitiviteit, specificiteit, positief voorspellende waarde, negatief voorspellende waarde en AUC van 38\%, 89\%, $56 \%$, $79 \%$ en 0.71 naar $60 \%, 93 \%, 64 \%, 92 \%$ en 0.95 . De AUC van de tweede beoordelaar verbeterde van 0.69 naar 0.79 . Derhalve werd gadofosveset-versterkte axillaire MRI beschouwd als veelbelovend non-invasieve methode voor (her)stadiëring van lymfeklieren.

Een feasibility studie met 18F-FDG hybride PET/MRI specifiek gericht op de axilla werd beschreven in hoofdstuk 6 . Twaalf klinisch klier positieve patiënten ondergingen axillaire echografie en axillaire hybride PET/MRI. Negen van de twaalf patiënten ondergingen tevens een PET/CT van het gehele lichaam. Axillaire hybride PET/MRI veranderde de klierstatus in vergelijking tot standaard beeldvormende technieken: $40 \%$ ten opzichte van axillaire echografie, $75 \%$ ten opzichte van T2 gewogen MRI, $40 \%$ ten opzichte van contrast-versterkte MRI en $\mathbf{2 2} \%$ ten opzichte van PET/CT. Concluderend, hybride PET/MRI specifiek gericht op de axilla is goed uitvoerbaar en zou de diagnostische performance van axillaire lymfeklierstadiëring kunnen verbeteren in klinisch klier-positieve patiënten.

\section{Deel III - Classificatie van lymfeklierstadiëring}

Hoofdstuk 7 onderzocht patiënten met een $\mathrm{pN}_{3}$ klierstatus (ten minste één infraclaviculaire lymfekliermetastase of $\geq$ tien axillaire lymfekliermetastasen). Er werd gebruik gemaakt van data van het Integraal Kankercentrum Nederland (IKNL). In een onderzoekspopulatie van 1788 patiënten met $\mathrm{pN}_{3}$ a werd aangetoond dat de prognose van patiënten met $\geq$ tien axillaire lymfekliermetastasen significant slechter was in vergelijking tot patiënten met ten minste één infraclaviculaire lymfekliermetastase met in totaal $\leq$ negen lymfekliermetastasen (ziektevrije overleving: $48.8 \%$ versus 63.8\%, $P=0.036$; totale overleving: $46.6 \%$ versus $63.9 \%, P=0.042$ ). Bovendien werd aangetoond dat de prognose van patiënten met ten minste één infraclaviculaire lymfekliermetastase vergelijkbaar is met patiënten met een pN2a klierstatus (vier tot negen axillaire lymfekliermetastasen) (ziektevrije overleving: $63.8 \%$ versus $67.3 \%, P=0.491 ; 63.9 \%$ 
versus $65.5 \%, P=0.233$ ). Revisie van de huidige classificatie van infraclaviculaire lymfkliermetastasen werd derhalve aanbevolen.

In hoofdstuk 8 werd de prognose van klinisch klier-positieve patiënten behandeld met neoadjuvante systemische therapie onderzocht, waarbij er een onderverdeling werd gemaakt op basis van ypN status: axillaire $\mathrm{pCR}$ (ypNo), residuale axillaire geïsoleerde tumorcellen of micrometastasen (ypNitc/mi) en residuale macrometastasen (ypN1-3). In een cohort van 1347 patiënten, verkregen via het IKNL, werd een vergelijkbare prognose aangetoond tussen ypNo en ypNitc/mi (ziektevrije overleving: Hazard Ratio (HR) 1.38 (0.40-4.79), P=0.613; totale overleving: HR 0.92 (o.27-3.09), $P=0.889)$. Prognose tussen ypNo en ypN1-3 was statisch significant verschillend (ziektevrije overleving: HR 1.78 (1.06-3.0o), $P=0.031$; totale overleving: $\mathrm{HR} 1.70(1.07-2.71), P=0.026)$. In conclusie, ypNo en ypNitc/mi zouden in toekomstige studies als één entiteit kunnen worden beschouwd.

Naast axillaire lymfekliermetastasen zijn parasternale lymfekliermetastasen prognostisch ongunstig. Geïsoleerde parasternale lymfekliermetasten, dus in de afwezigheid van axillaire lymfekliermetastasen, zijn volgende de huidige TNM classificatie onderverdeeld op basis van methode van detectie: tijdens de schildwachtklierprocedure $\left(\mathrm{pN}_{1} \mathrm{~b}\right)$ of tijdens klinisch onderzoek ( $\left.\mathrm{pN} 2 \mathrm{~b}\right)$. In hoofdstuk 9 wordt echter geen prognostisch verschil gevonden tussen beide subgroepen, waarbij de onderzoekspopulatie bestaat uit 101 patiënten met geïsoleerde parasternale lymfekliermetastasen, verkregen via het IKNL. Na multivariabele cox regressieanalyses betreft ziektevrije overleving: HR o.29 (o.o42.33), $P=0.244$ en totale overleving: HR 1.04 (o.37-2.89), $P=0.947$. Derhalve zouden beide subgroepen wellicht als één categorie kunnen worden beschouwd. 

Valorisation 



\section{Valorisation}

Breast cancer is the most common type of invasive cancer among women worldwide, with an estimated 1.7 million cases and 521,900 deaths in 2012. In the Netherlands, the annual incidence is more than 14,000 patients. Nowadays, standard treatment consists of surgery of the breast and axilla, complemented with radiation and/or systemic therapy. This extensive treatment has led to a mean five year overall survival of $90 \%$. Regarding the health care costs, breast cancer treatment represents approximately $15 \%$ of all health care costs in the Netherlands.

The excellent survival caused an attention shift from survival to the presence of morbidity and quality of life after treatment. Better patient selection for specific therapies and use of less invasive techniques, in order to avoid overtreatment on an individual level, aim to further minimize morbidity and complications of therapy and consequently reduce nationwide health care costs.

The aim of this thesis was to investigate the enigma of lymph node staging in breast cancer patients, by improving diagnostic accuracy of nodal staging in order to achieve a more patient-tailored treatment with minimal invasive therapy and reduced morbidity.

\section{Relevance of scientific results in this thesis}

Currently, axillary surgery is performed in every patient to investigate the axillary lymph node status. In clinically node negative patients sentinel lymph node biopsy (SLNB) is performed, whereas axillary lymph node dissection (ALND) is performed in clinically node positive patients. These procedures can cause complications, such as lymphedema, numbness and pain. In addition, patients without axillary lymph node metastases do not benefit from the axillary surgery, since they do not have any prognostic benefit from the procedure. Approximately $80 \%$ of clinically node negative patients and $40 \%$ of clinically node positive patients treated with neoadjuvant systemic therapy only have benign axillary lymph nodes. Yet, these patients are still exposed to axillary surgery with its concomitant complications.

This thesis explored further techniques to improve lymph node staging in breast cancer. We showed that patients with negative axillary findings on $\mathrm{T}_{2}$ weighted breast MRI, with a complete field of view of the axillary region, do not require any further diagnostic imaging. When compared to axillary ultrasound, T2 
weighted breast MRI even improved differentiation between limited and advanced axillary nodal disease.

In clinically node positive patients treated with neoadjuvant systemic therapy, RISAS-procedure might become the new standard procedure to accurately identify axillary pCR. Previous studies already demonstrated less complications after SLNB as opposed to ALND. Consequently, ALND can be safely omitted in patients with axillary $\mathrm{pCR}$ according to RISAS, indicating these patients will experience less often any of the aforementioned surgical complications. Yet, final results of this Dutch prospective multicenter trials must confirm the validity of this technique first.

Finally, this thesis demonstrated that dedicated axillary 18F-FDG hybrid PET/MRI is feasible in clinically node positive breast cancer patients to differentiate between limited and advanced axillary nodal disease. However, results of this technique should be further explored in research setting, before implementation into daily clinical practice is justified.

\section{Target population}

This thesis contributes to newly diagnosed breast cancer patients, by improving the diagnostic accuracy of axillary lymph node staging. In addition, results of this thesis are relevant for all physicians involved in treatment trajectories of breast cancer patients. Finally, dedicated axillary hybrid PET/MRI might encourage other researchers in the field of oncologic imaging in which lymph node staging is an important prognostic factor, such as melanoma, esophageal, ovarian, prostate or rectal cancer.

\section{Innovation and future}

Future studies should explore whether increasingly advanced imaging tools, like dedicated axillary hybrid PET/MRI, can accurately exclude lymph node metastases in clinically node negative patients, making SLND a redundant operation in node negative patients. Consequently, node negative patients would no longer harm from axillary surgery. In addition, cost-effectiveness of implementation of dedicated axillary hybrid PET/MRI should also be investigated.

Since the introduction a few years ago, hybrid PET/MRI has been incorporated in daily clinical practice for several (neurologic) indications. So far, no research has been performed investigating the feasibility of dedicated axillary hybrid PET/MRI to improve lymph node staging in breast cancer. This thesis demonstrated that dedicated axillary hybrid PET/MRI for lymph node staging is 
feasible and it changed nodal status when compared to current imaging modalities.

Besides, diagnostic performance of dedicated axillary hybrid PET/MRI after neoadjuvant systemic therapy in clinically node positive patients should be investigated to determine whether this technique can accurately identify axillary pCR. This would allow patients who converted to axillary pCR to omit any further axillary surgery, which would be even more beneficial compared to RISASprocedure. 
